\section{Oral Communication}

\section{Abstracts}

Cite this article: (2020). EPA 2020 Abstracts, Part 2. European Psychiatry 63(S1), S629-S712. https://doi.org/10.1192/j.eurpsy.2020.95

\section{0}

(c) The Author(s), 2020. Published by Cambridge University Press on behalf of The European Psychiatry Association. This is an Open Access article, distributed under the terms of the Creative Commons Attribution licence (http:// creativecommons.org/licenses/by/4.0/), which permits unrestricted re-use, distribution, and reproduction in any medium, provided the original work is properly cited.

\section{Construct and concurrent validity of illness and help-seeking behaviour scale} (IHSBS) in portuguese mental health patients

\author{
A. Macedo, C. Silva*, M.J. Soares, A. Araújo, A.T. Pereira, N. Madeira, D. Moura, M. Coroa, \\ S. Morais, I. Rosendo and A.F. Miranda \\ Portugal \\ ${ }^{\star}$ Corresponding author.
}

Introduction: Illness behaviour and Help-Seeking behaviour can have great impact on patient's health and quality of life; therefore, it's important to have an appropriate scale to assess these constructs.

Objectives: To study the psychometric qualities of the IHSBS on Portuguese mental health patients, its reliability, its construct and concurrent validity, measured using the Stigma Scale (SS). Methods: The sample comprised 140 mental health patients, with different psychiatric diagnosis (70\% women), with mean age of 39.49 years old ( $\mathrm{SD}=15.71 ; \mathrm{V}=18-78)$, followed in health centers and hospitals in the central area of the country. They filled out a questionnaire that included the SS and the IHSBS.

Results: The KMO was .681 and Bartlett's test $\mathrm{p}<.05$. The analysis of the main components and scree plot revealed 3 components which was confirmed by Horn's parallel analysis. The three factors were submitted to a varimax rotation - F1 is Help-Seeking Behaviour (EV=17.97\%; $\alpha=.69)$, F2 Health Worries (EV=15.55\%; $\alpha=.76$ ), F3 Illness Behaviour (EV=9.01\%; $\alpha=.68)$, all had acceptable internal consistency and so did the total scale $\mathrm{EV}=42.53 \% ; \alpha=.64)$. Four items were excluded: items 9, 16, 3 had inacceptable loadings in all dimensions and item 2 didn't contribute for its sub-scale internal consistency. The correlation between IHSBS total score and its sub-scales ranged from .36 to $.76(\mathrm{p}<.01)$; between F2 and the SS was $.37(\mathrm{p}<.01)$ and between F3 and the SS was .19 $(\mathrm{p}<.05)$.

Conclusions: The IHSBS is a valid and reliable instrument, that can be useful to assess Illness and Help-Seeking behaviour in mental health patients.

\section{Conflict of interest: No \\ Keywords: IHSBS; Stigma; mental health}

\section{8}

Neurotoxic effects of varying doses of tramadol on the cerebral cortex of adult male wistar rats

B. Adebisi*

Nigeria

${ }^{\star}$ Corresponding author.

Introduction: Tramadol is a centrally acting opiod analgesic used in the treatment of moderate to severe pain.There has been some concerns regarding the dependence liability of the long-term use of the drug. However, this present study is conducted to assess the histological toxicity profiles of long term use of Tramadol, in which the cerebral cortex is studied in adult male wistar rats. Objectives: The objective of the study aimed at validating the cognitive and behavioural disorientations reported in humans, in addictive and long term use of Tramadol, by experimentation and histological representation of the toxic effects on the cerebral cortices of rats, treated with different doses of the opioid.

Methods: Twenty adult male wistar rats were subjected to experiment for sixty days. They were divided into four groups (A, B, C, and D). Group A serves as the control group and the rats in the group were administered with normal saline, while Groups $\mathrm{B}, \mathrm{C}$, and D received the dosages of Tramadol at 20, 30 and $40 \mathrm{mg} / \mathrm{kg}$ body weight respectively, daily, for sixty consecutive days. Thereafter, the animal were sacrificed, their brain excised and the brain tissues were processed for histological procedure and assesment.

Results: Histology of brain tissues show varying extents of disorientations; damaged fibers, stunting cell processes of neurons, increase glial cell population and distorted cortical layers were some of the features seen on the cerebral cortex histology. 
Conclusions: The toxic effects of Tramadol is evident on the Cerebral cortex and should be kept in mind, even durng prescriptions by medical personnels.

\section{Conflict of interest: No}

Keywords: Cerebral cortex; Neurotoxic; tramadol; Neuronal dysfunction

\section{0}

\section{Associations between thyroid hormone levels and clinical outcomes in schizophrenic inpatients}

M.J. Jung*, S.H. Lee, T.K. Lee and J.H. Choi

Korea, Republic of

${ }^{\star}$ Corresponding author.

Introduction: Thyroid hormone affects brain development and deficiency of this hormone can induce psychiatric symptoms. This shows a possible relationship between thyroid hormone and schizophrenia.

Objectives: We examined the association between thyroid hormone levels and clinical outcomes in schizophrenic inpatients.

Methods: Schizophrenic inpatients with no thyroid disease were studied. We assessed blood thyroid hormone levels and BPRS scores in admission period. The presence of social security disability because of schizophrenia and the use of clozapine were also assessed. Collected data were analyzed using T-test and multiple regression analysis.

Results: Three hundred thirty-eight patients were studied (Table 1). No significant difference in mean age or gender ratio between groups was seen. The group with no treatment response and social security disability showed lower free T4 levels $(\mathrm{P}<0.05)$ (Table 2). Conclusions: Free T4 level was significantly associated with treatment response and the presence of social security disability from schizophrenia. These relationships suggested that thyroid hormone may be associated with the clinical outcomes of schizophrenia and further analysis is required.

Table 1 Demographic and clinical characteristics

\begin{tabular}{ll}
\hline $\mathrm{N}=338$ & Mean \\
\hline Age (years) & 49.6 \\
\hline BPRS score reduction rate (\%) & 13.0 \\
\hline & $\%$ \\
\hline Male & 54.4 \\
\hline Clozapine use & 16.0 \\
\hline Social security disability & 51.8 \\
\hline
\end{tabular}

\section{Conflict of interest: No}

Keywords: Thyroid hormones; schizophrenia; Clinical outcomes; social security disability

\section{7}

\section{Analysis of microrna expressions in schizophrenia: Insights into molecular interactions and functional associations underpinning neurodevelopmental processes}

\author{
B.-Y. Chen*, J.-J. Lin, M.-K. Lu and S.-H. Lin \\ Taiwan \\ ${ }^{*}$ Corresponding author.
}

Introduction: MicroRNA (miRNA) can be regarded as one of master regulators of neurodevelopment that displays genetic and epigenetic roles that could therefore be affected by disease processes. However, the differences of neurodevelopment-related miRNAs expression levels in schizophrenia patients, nonpsychotic relatives of schizophrenia patients and bipolar disorder patients remain unclear.

Objectives: In this study, we aimed to investigate the aberrant expression of miR-137 and miR-34 family in patients with schizophrenia, then using canonical pathways analysis to identify neurodevelopment-related miRNAs' molecules roles and functions.

Methods: In the current study, we collected blood samples of patients with schizophrenia $(\mathrm{n}=215)$ and nonpsychotic controls $(\mathrm{n}=100)$ as comparison groups. We examined the microRNAs expression levels in the different groups by receiver operating characteristic (ROC) curve and partial least squares-linear discriminant analysis (PLS-LDA). Moreover, the results of microRNAs expressions were also uploaded into ingenuity pathway analysis system for core analysis and then overlaid with the canonical pathways analysis.

Results: The miR-137 and miR-34 family expression levels presented significant expression patterns that could distinguish schizophrenia from nonpsychotic controls ( $p>0.001)$. Annotating results from real-time polymerase chain reaction ( $\mathrm{qPCR}$ ) experiments are L-DOPA degradation $(-\log (\mathrm{p}$-value $)=2.81)$ and dopamine receptor signaling $(-\log (\mathrm{p}$-value $)=2.80)$. The bioinformatics results obtained through enrichment analyses to map miR-137 and miR-34 family related information with schizophrenia $(\mathrm{p}=4.69 \mathrm{E}-9)$ and brain lesion ( $\mathrm{p}=1.29 \mathrm{E}-8)$ for disease annotations.

Conclusions: The findings provide further insights into the neurodevelopment-related miRNAs expression levels as potential peripheral blood biomarkers for schizophrenia.

Table 2 Comparison of mean value of thyroid hormone level between

\begin{tabular}{|c|c|c|c|c|c|c|c|c|c|}
\hline & \multicolumn{3}{|c|}{ Treatment response } & \multicolumn{3}{|c|}{ Social security disability } & \multicolumn{3}{|c|}{ Use of clozapine } \\
\hline TSH & 1.50 & 2.45 & .175 & 2.61 & 1.79 & .172 & 1.76 & 2.30 & .511 \\
\hline Total T3 & 1.10 & 1.05 & .108 & 1.05 & 1.07 & .336 & 1.01 & 1.07 & .104 \\
\hline Free T4 & 1.38 & 1.25 & $.001^{*}$ & 1.25 & 1.32 & $.029^{\star}$ & 1.26 & 1.29 & .553 \\
\hline
\end{tabular}




\section{Conflict of interest: No}

Keywords: schizophrenia; microRNA; neurodevelopment; nonpsychotic first-degree relatives; bipolar disorder

\section{8}

The relation between changes in vitamin $D$ and vitamin B12 levels, treatment characteristics, and outcome in methadone maintenance treatment patients

\author{
E. Malik ${ }^{\star}$, L. Rozner, M. Adelson, S. Schreiber and E. Peles \\ Israel \\ ${ }^{\star}$ Corresponding author.
}

Introduction: Substance use disorder is a major socioeconomic concern, associated with malnutrition and low plasma vitamin levels. Vitamin B12 and vitamin D deficiency are prominent cause for morbidities. So far, there have been conflicting reports about an increase in BMI levels in patients under MMT.

Objectives: We aimed to study vitamins D and B12 deficiency and changes over time with respect to treatment characteristics and outcome in patients with opioid use disorder receiving methadone maintenance treatment (MMT).

Methods: 272 patients had documented vitamin B12 levels and 260 had patients had documented vitamin D levels, of them 112 and 80 patients respectively had two measures (at admission or while already in treatment, and while stabilized or after one year in treatment). BMI (calculated by weight and height) and long-term retention were analyzed.

Results: Vitamin B12 was lower in patients with positive urine for cocaine/amphetamine on admission. Vitamin D did not change over time, but in 38 patients whose vitamin D was elevated as compared to 42 patients whose level was not, a significant weight gain could be observed $(25.4 \pm 4.8$ to $28.8 \pm 5.2$ vs. $24.3 \pm 3.7$ to $25.5 \pm 4.0, \mathrm{p}$ (Time $)<0.0005, \mathrm{p}($ Group $)=0.03, \mathrm{p}($ interaction $)=0.02)$. $\mathrm{BMI}$ change correlated with vitamin $\mathrm{D}$ change $(\mathrm{R}=0.26, \mathrm{p}=0.04)$. Longer cumulative retention was observed among the elevated vitamin D group (8.1 years, 95\% CI 6.3-9.8) as compared to nonelevated group (4.8y 95\% CI 3.6-6.1, Kaplan Meier chi square 5.2, $\mathrm{p}=0.02$ ).

Conclusions: Stimulants misuse was associated with low B12 levels. Vitamin D elevation is associated with weight gain and longer retention in treatment.

\section{Conflict of interest: No}

Keywords: Methadone maintenance treatment; Body mass index (BMI); Vitamin D; Vitamin B12

\section{8}

Gender differences in body size perception, dieting and mental distress amongst adolescents, the nord-trøndelag health study (YH3)

\author{
F. Sardahaee*, T. Holmen and K. Kvaløy \\ Norway \\ ${ }^{\star}$ Corresponding author.
}

Introduction: Worldwide increase in adolescent obesity is in stark contrast with super thin ideal body size defined by modern societies. Dieting has been widely promoted as a reliable method for maintaining ideal body weight(and shape) despite ample evidence on their in-effectivity and harmfulness to physical and mental wellbeing. Dieting is more prevalent amongst adolescent girls than boys.

Objectives: Given that societal definitions of ideal body shape are gender based, we investigated gender differences in exhibiting mental distress given adolescents' body size perception.

Methods: Multinomial logistic regression was employed to test for associations between BMI, body size perception, mental distress and dieting in data drawn on 7,718 individuals (aged 13-19) from Young HUNT 3 survey. Analyses were done in gender stratified populations and were adjusted for age and socioeconomic status (SES).

Results: Prevalence of dieting was much less in boys than girls (9\% Vs. 21.6\%) despite higher prevalence of overweight amongst boys. Although girls who perceived their body size as "not normal" showed a greater increase in OR for dieting (OR:37 in girls Vs. OR: 26 in boys, p-value $<0.001$, CI 95\%), ironically it was boys who showed a disproportionately higher levels of mental distress $(\mathrm{RR}>1.2)$ if they perceived their body as "fat". Similar trend was observed amongst boys who perceived themselves as "thin".

Conclusions: Our findings call into question the generally held belief that compared to girls, boys are less distressed by having a self perceived abnormal body size perception. Further research is needed to investigate what constitutes this male vulnerability.

\section{Conflict of interest: No}

\section{9}

Major depressive disorder is associated to increased levels of proinflammatory cytokines and alterations in monocytes

M. Alvarez-Mon*, A. Gomez, A. Orozco, G. Lahera, M.D. Sosa, E. Auba, A. Albillos and M. Alvarez-Mon

Spain

${ }^{*}$ Corresponding author.

Introduction: Pathogenesis of Major Depressive Disorder (MDD) is still partially unknown. MDD is associated with a chronic, lowgrade inflammatory response.

Objectives: investigate the intracytoplasmic production of TNFa, IL-1B, IL- 6 and IL-10 after LPS stimulation and the serum levels of these cytokines.

Methods: We included 22 patients with the following inclusion criteria: 1) Psychiatrist-confirmed diagnosis of MDD, single or recurrent, according to the Diagnostic and Statistical Manual of Mental Disorders criteria (DSM-V);2) A minimum score of 14 points on the 17-item Hamilton Rating Scale for Depression (HRSD). We also included 14 matched healthy controls (HCs).

Results: MDD patients had significantly higher serum levels of TNFa and IL-1B than HC. The percentage of the total monocyte population that expressed IL-1B in MDD was significantly higher than in HC (Figure 1). MDD patients also had a significantly increased percentage of classical monocytes that expressed IL-6 (Figure 2). 

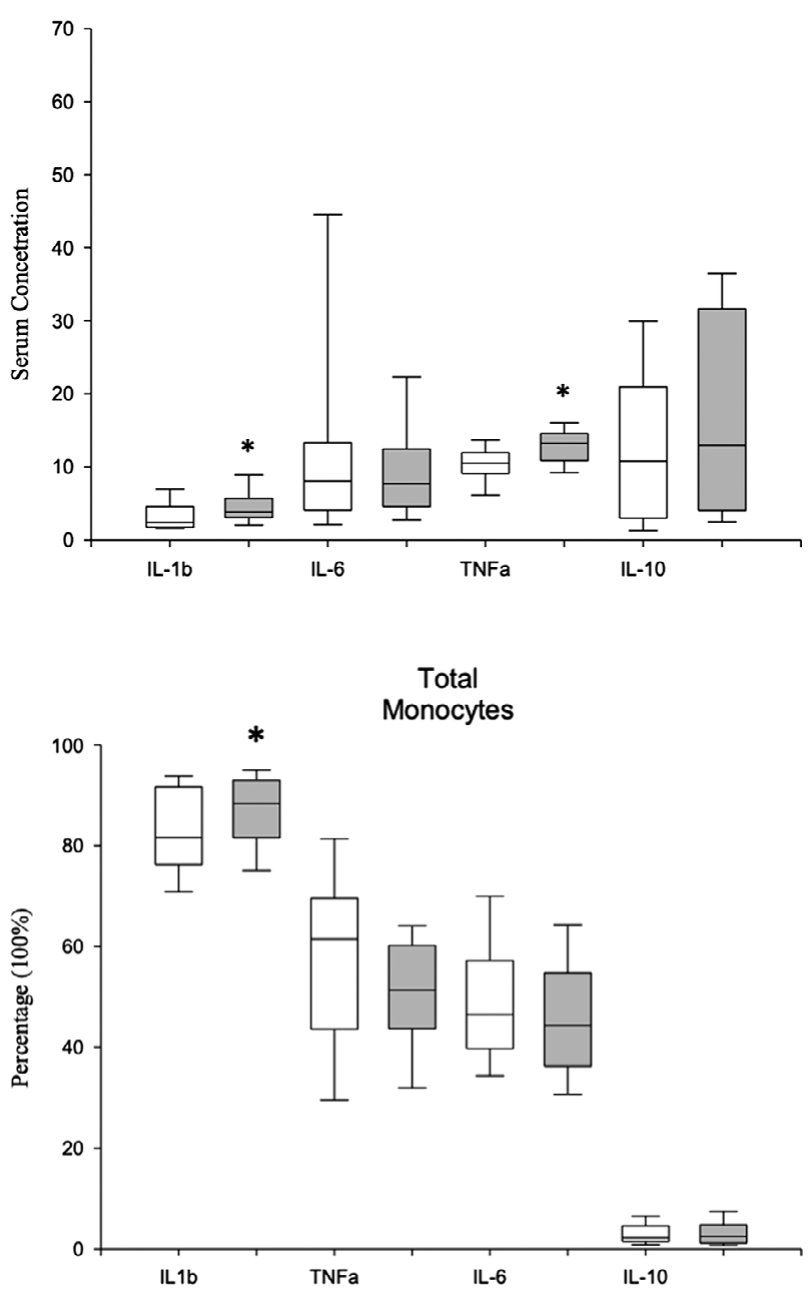

Conclusions: MDD patients showed increased IL-1B and IL-6 expression as well as a systemic state characterize by increased serum levels of TNFa and IL-1B.

\section{Conflict of interest: No}

Keywords: major depressive disorder; Monocytes; cytokines; systemic inflammation

\section{E-Poster Presentations}

\section{Addictive disorders - Part I}

\section{EPP0001}

Prevalence of substance use disorder in asperger's - a case report and review of literature

Z. Ahmad $^{1 *}$, S. Gunturu ${ }^{2}$, R. Shengelia ${ }^{3}$, A. Zamiri ${ }^{3}$ and O. Korenis ${ }^{4}$

${ }^{1}$ BronxCare Hospital Center, Psychiatry, Bronx, United States of America; ${ }^{2}$ BronxCare Hoospital, Psychiatry, Bronx, United States of America; ${ }^{3}$ BronxCare Health System, Psychiatry, Bronx, United States of America and ${ }^{4}$ BronxCare, Psychiatry, Bronx, United States of America

${ }^{\star}$ Corresponding author.

Introduction: Asperger syndrome is a neurodevelopmental disorder that is characterized by significant impairment in reciprocal social communication and nonverbal interaction. It was considered a milder or higher functioning form of autism spectrum disorder as it did not feature significant language or intellectual impairment (1). Asperger's syndrome is estimated to impact around 0.26 per 1,000 people (2).

Objectives: The main objective of our research was not only to evaluate the prevalence of substance use disorders in people suffereing from Asperger's syndrome but also to potentially devise methods to better address such problems in future.

Methods: We present a 22-year-old male, with a psychiatric history of Asperger's syndrome, major depressive disorder, generalized anxiety disorder and co-existing substance use history of Alcohol, Benzodiazepines, and anti-histaminergic medications. In our poster: We discuss the hospital course, the biopsychosocial formulation, and discharge planning for this patient followed by review of Literature.

Results: Despite previous perception of lower substance use disorders among adults with Asperger's syndrome due their introverted and somewhat inhibited nature, (3), further research has shown that this population may actually have a greater propensity towards substance use disorder. The possible rationale includes the use of illicit substances to overcome different deficiencies in social interactions. and potentially self-medicate psychiatric comorbidities such as depression, Obsessive-compulsive disorder and insomnia (4). Around $65 \%$ of patients with Asperger's syndrome are estimated to have a comorbidity of Anxiety and Depression (5).

Conclusions: Finally, we conclude by giving some clinical pearls on recognizing, assessing, managing and treating substance use in Aperger's disorder patients.

Conflict of interest: No

Keywords: substance use disorder; Autism Spectrum Disorder; dual mental and substance use disorder; Asperger syndrome

\section{Addictive disorders - Part III}

\section{EPP0034}

\section{Relapse after alcohol detoxification in an acute psychiatric ward: a tertiary care center based cross-sectional study}

J. Gonçalves Cerejeira*, I.D.L.M. Santos Carrasco, C. Capella, E. Rodríguez, M. De Lorenzo, N. De Uribe, M. Gómez, M. Queipo De Llano, A. Gonzaga, G. Guerra and O. Santiago

Hospital Clinic of Valladolid, Psychiatry, Valladolid, Spain ${ }^{\star}$ Corresponding author.

Introduction: Many patients request hospital admission to initiate alcohol detoxification, but after discharge relapse is frequent. Consistent studies showing the specific clinical predictors are still missing. Objectives: To describe the characteristics of the patients admitted to alcohol detoxification and the factors associated with a relapse in the months following discharge.

Methods: This is a cross-sectional study. We collected data of the patients admitted to our acute hospital ward during the year 2018 from the medical records in the hospital information system, as well as data from subsequent 9-month follow-up period after discharge.

Results: 35 patients requested admission to start an alcohol detoxification program. Seventeen patients (49\%) relapsed within nine 
months after discharge. Patients with a previous history of alcohol detoxification [14/26 (54\%)] and with a comorbid substance use disorder $[5 / 8(63 \%)]$ relapsed more than patients without it $[3 / 9$ (33\%) and 12/27 (44\%)]. Similarly, patients with a co-occurring psychiatric disorder relapsed more than patients without comorbidity [11/16 (69\%) against a $6 / 19(32 \%)]$. On the other hand, patients who started a long-term recovery program $[4 / 13(31 \%)]$ or those who received drugs for alcohol use disorder [6/17 (35\%)] relapsed less than patients who didn't [10/17 (59\%) and 11/18 (61\%)].

Conclusions: Previous history of alcohol detoxification, comorbid substance use disorder and co-occurring psychiatric disorder could predict a higher relapse rate. Long term recovery programs and pharmacological treatment could predict lower relapse rates.

Conflict of interest: No

Keywords: relapse; alcohol use disorder

\section{Addictive disorders - Part VII}

\section{EPP0067}

\section{Complications in chronic alcoholism: extrapontine myelinolysis}

R. Torralba Viorreta ${ }^{1 \star}$, A. Gomez-Peinado ${ }^{2}$, V. Quero-Palomino ${ }^{1}$ and S. Martínez Larumbe ${ }^{3}$

${ }^{1}$ Hospital General Universitario de Ciudad Real, Psychiatry, Ciudad Real, Spain; ${ }^{2}$ Hospital General Universitario de Ciudad Real, Psiquiatría, Ciudad Real, Spain and ${ }^{3}$ Hospital Universitario Ramón y Cajal, Psychiatry, Madrid, Spain

${ }^{\star}$ Corresponding author.

Introduction: Primary polydipsia has been found in several mental disorders and can lead to serious complications such hyponatremia. When hyponatremia is too rapidly corrected, can produce pontine or extrapontine myelinolysis, which have behavioural and neurological manifestations that often are irreversible. Alcoholism is a risk factor for chronic hyponatraemia and for extrapontine myelinolysis. It is important to prevent those syndromes when treating patients with alcohol use disorder and polydipsia.

Objectives: To remark extrapontine myelinolysis as a possible complication in patients with alcohol use disorder.

Methods: Report of a real clinical case and bibliographic research. Results: We describe the case of a 55-year-old woman with alcohol use disorder who is admitted to the hospital after being found at home with low consciousness. At emergencies, a state of malnutrition was detected, with hyponatremia ( $\mathrm{Na} 96 \mathrm{mEq} / \mathrm{L})$ and hypokalaemia (K $3 \mathrm{mEq} / \mathrm{L})$. In previous months she had been drinking 10-15 L of water everyday. She was diagnosed with primary polydipsia and treated with hypertonic saline serum, with rapid improvement of electrolyte balance. After 4 days, the patient showed weakness, parkinsonism, dysatria, dysphagia, sialorrhea, affective lability, desinhibited and impulsive behaviors and hyperorality. MRI brain revealed bilaterally symmetric hyperintensity of signal with FLAIR in striated nuclei, with restriction of associated diffusion. She was diagnosed with extrapontine myelinolysis. After 2 months those symptoms persist.

Conclusions: Patients with chronic alcoholism are in high risk for developing pontine or extrapontine myelinolysis. Pontine and extrapontyne myelinolysis treatment is based on prevention, so clinitians must know this risk.
Conflict of interest: No

Keywords: hyponatremia; extrapontine myelinolysis; alcohol use disorder

\section{EPP0076}

Screening for alcohol-related cognitive impairments - a case study

L. Gil ${ }^{1}$, M. Lázaro $^{2}$, S. Ferreira ${ }^{3}$, P. Diegues ${ }^{3}$, J. Teixeira $^{3}$ and T. $\operatorname{Mota}^{3}$

${ }^{1}$ Centro Hospitalar Psiquiátrico de Lisboa, Clínica 6, Lisbon, Portugal; ${ }^{2}$ Centro Hospitalar Psiquiátrico de Lisboa, Clínica 5, Lisboa, Portugal and ${ }^{3}$ Centro Hospitalar Psiquiátrico de Lisboa, Serviço Alcoologia E Novas Dependências, Lisboa, Portugal

${ }^{*}$ Corresponding author.

Introduction: Harmful effects of chronic alcohol excessive consumption on the brain and cognitive functioning have been described, but are largely underestimated in clinical practice. Such cognitive disorders can interfere with the motivation process to abandon maladjusted drinking behavior and limit patient's capacity to benefit from treatment, contributing to relapse.

Objectives: Theme review on the available evidence regarding alcohol-related cognitive impairments. Describe the major cognitive impairments in a group of patients diagnosed with mental and behavioral disorder due to use of alcohol.

Methods: Non-systematic review of the literature was performed in PubMed/Medscape database. Characterization of a group of patients diagnosed with mental and behavioral disorder due to use of alcohol with cognitive complaints. Montreal Cognitive Assessment (MoCA) was applied.

Results: Research about alcohol-related cognitive impairments is challenging, considering multiple patterns of alcohol abuse, personal and lifestyle factors, and vulnerability of specific brain regions. We studied 9 patients diagnosed with mental and behavioral disorder due to use of alcohol with cognitive complaints affecting daily routine. They were included in a relapse prevention program after abstinence. In an initial assessment, MoCA was applied and 8 patients showed results under the cut-off point, being the mainly affected domains verbal fluency, memory, attention and visuospatial capacities. Patients were included in a Neuromodulation Cognitive Program in Centro Hospitalar Psiquiátrico de Lisboa.

Conclusions: Our research revealed significant cognitive deficits in this group of patients, highlighting the importance of recognizing and screening for alcohol-related cognitive impairments, that are largely underestimated in clinical practice and have large implication in treatment and prognosis.

Conflict of interest: No

Keywords: Alcohol dependence; Cognitive impairment; remediation; relapse

Anxiety disorders and somatoform disorders - Part II

\section{EPP0095}

Migraine comorbidity with anxiety disorder, a study in regional hospital durres, Albania

E. Shemsi $(\text { Harizi })^{1 \star}$, K. Shemsi ${ }^{2}$ and F. Domi ${ }^{3}$ 
${ }^{1}$ Regional Hospital Durres, Neurology, Quarttier Street A.Goga, Albania; ${ }^{2}$ University of Medicine Tirana, Faculty Of Medicine, Tirana, Albania and ${ }^{3}$ Regional Hospital Durres, Emergency, Durres, Albania ${ }^{\star}$ Corresponding author.

Introduction: Patients with migraines have symptoms of anxiety more than other patients. About $50 \%$ of patients with migraine have anxiety. This comorbidity between migraine and anxiety is because of brain chemicals involved of serotonin and some hormone changes in women, that stimulate both conditions.

Objectives: Symptoms of anxiety are common in patients with chronic migraine. These patients have less response to headache medications and have more relapse. Symptoms such fatigue, trouble concentrating, appetite, and sleep changes are common.

Methods: In our study we have seen 400 patients with migraine in ambulatory policlinic in SRD in year 2017. 330 (82.5\%) patients were female and $70(27.5 \%)$ male. From all patients 208 (52\%) have migraine accompanied with anxiety disorder (180 female $(86.5 \%)$ and 28 male $(13.5 \%)$ ). Middle age of patients with migraine and anxiety was 32.6 years and for patients without anxiety was 38.1 year. All patients of migraine and anxiety had preventive therapy with amytriptiline $25 \mathrm{mg}$ per day for a group of them, and escitalopram 20 md per day for others (for long time treatment 6 - 12 months).

Results: The mean frequency of crisis in patients with anxiety was 15.4 days and without anxiety 4.3 days a month. After treatment of anxiety for one year the mean frequency of crisis was lower 13.6 days.
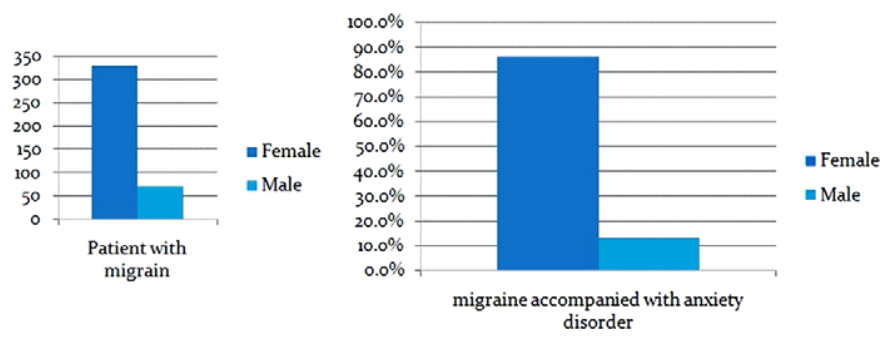

Conclusions: We need to understand better the impact of treating anxiety in patients with headache comorbidity because so we can to obtain best treatment for each disorder. Effective drugs and behavioral therapies are available to have good results and less crisis of migraine.

Conflict of interest: No

Keywords: Migraine; Comorbidity; Anxiety; Disorder

\section{Bipolar disorders - Part I}

\section{EPP0102}

\section{Impact of childhood trauma in patients with bipolar disorder.}

M. Betriu*, A. Guàrdia Delgado, G. Navarra, M. Serra-Blasco, C. Massons, S. Crivillés, M. Guinovart, J.A. Monreal, D. Palao Vidal and N. Cardoner Ávarez

Parc Taulí University Hospital. Autonomous University of Barcelona (UAB). I3PT. CIBERSAM, Department of Mental Health, Sabadell, Spain

${ }^{\star}$ Corresponding author.
Introduction: A growing number of studies support that subjects who have experienced Childhood Trauma (CT) show deficits in general cognitive performance. However, this correlation has been scarcely studied in patients with bipolar disorder.

Objectives: Our aim is to examine the potential association of CT on cognitive performance in euthymic patients diagnosed with bipolar disorder.

Methods: We conducted a cross-sectional study on a sample of 64 bipolar outpatients recruited from the Bipolar Disorder Program of the Parc Taulí University Hospital in Sabadell (Spain). Participants underwent a comprehensive clinical interview, which specifically included the Child Trauma Questionnaire (CTQ) and a cognitive evaluation with a battery of neuropsychological tests. Inclusion criteria were as follows: (a) bipolar I or II (DSM 5) diagnosis, (b) age $>18$, (c) fulfilling euthymia criteria for at least 3 months, (d) the capacity to provide written informed consent. Exclusion criteria were: (a) IQ < than 80 (b) a course of electronvulsive therapy within the past year, (c) substance abuse, (d) medical condition affecting neuropshychological performance. No restrictions were made regarding pharmacological treatment. A two-step cluster analysis will be applied to delimit different groups of bipolar patients based on the presence of $\mathrm{CT}$ and their clinical features and cognitive performance.

Results: Our preliminary results suggest that the history of child abuse measured by CTQ is associated with a worse performance in different cognitive domains.

Conclusions: Exposure to CT has lasting consequences in the clinical presentation of bipolar disorder; fact that reinforces the need to establish specific prevention and treatment strategies.

Conflict of interest: No

Keywords: Bipolar disorder; cognitive performance; childhood trauma; clinical features

\section{EPP0103}

Gut feeling: the connection between gut microbiota and bipolar affective disorder

\section{J. Brás*, R. Sousa, B. Guedes, A. Costa and A. Costa}

Centro Hospitalar Tondela-Viseu, Department of Psychiatry and Mental Health, VISEU, Portugal

${ }^{\star}$ Corresponding author.

Introduction: Bipolar affective disorder $(\mathrm{BAD})$ is a psychiatric disease characterized by changes in the mood and energy. BAD comprises depressive and manic/hipomanic episodes. The pathogenesis of $\mathrm{BAD}$ is not fully understood. However, interactions between genetic, environmental and biologic factors may play a role. Recent studies identified interactions between the gut and the brain, throughout neuroanatomical, neuroimmune and neuroendocrine pathways. Furthermore, gut microbiota's alterations were observed in several neuropsychiatric conditions, particularly in BAD.

Objectives: To present a non-systematic review of the literature examining the gut microbiota composition in BAD patients.

Methods: A literature search was conducted using the search engine Pubmed ${ }^{\circledR}$ and the keywords: gut microbiota and bipolar affective disorder.

Results: A variety of studies showed differences in gut microbiota's composition and diversity in BAD patients. Higher abundance of the Bacteroidetesphylum was observed by some authors. The phylum Firmicutesis more abundant in healthy individuals, however some species are increased in BAD patients. At genus level, the studies identified an higher prevalence of some genera, specifically 
Parabacteroides, Bacteroides, Weissela, Halomonas and Oscillibacter. The results about the genus Bifidobacterium are ambiguous. Some authors defended that probiotics improve mood, stress and anxiety, while other identified a higher number of Bifidobacterium in BAD patients. Moreover, compared to healthy individuals, decreased abundance of various butyrate-producing bacteria was found in BAD patients.

Conclusions: Significant alterations are present in gut microbiota of $\mathrm{BAD}$ patients. These alterations may clarify some aspects related to pathophysiology, diagnosis and treatment of bipolar disease. Nevertheless, more studies are necessary to better understand these differences.

\section{Conflict of interest: No}

Keywords: Bipolar disorder; gut microbiota; gut-brain axis; Inflammation

\section{EPP0104}

A clinical overview of bipolar disorder among voluntary and involuntary admitted patients in a psychiatric service during 2018: clinical differences and similarities

F. Caldas ${ }^{1}$, P. Frias ${ }^{1}$, R. Valido ${ }^{1}$, M. Barros ${ }^{1}$, D. Pires ${ }^{1}$, M. Gonçalves ${ }^{2}$ and M. Falcão

${ }^{1}$ Hospital de Magalhães Lemos, Psychiatry, Porto, Portugal; ${ }^{2}$ Hospital de Magalhães Lemos, Psychiatry, porto, Portugal and ${ }^{3}$ Centro Hospitalar do Porto, Centro Materno Infantil Do Norte, porto, Portugal

${ }^{\star}$ Corresponding author.

Introduction: Bipolar Disorder (BD) is a chronic and severe mental disorder, characterized by acute episodes that very frequently require hospitalizations. Compulsory admission is the last line of intervention in individuals who suffer from severe mental disorders and refuse treatment, based on the principles of therapeutic need and social protection.

Objectives: Retrospective and descriptive study to outline statistically significant differences (SSD) across socio-demographic, clinical and psychopathological variables of voluntary and compulsory (VC) hospitalizations among patients with BD.

Methods: Charts of all patients with a diagnosis of BD admitted in Hospital Magalhães Lemos (HML) during 2018 were reviewed to gather data. Statistical analysis of data with "SPSS21".

Results: In 2018, a total of 2890 patients were hospitalized in HML, 138 of which diagnosed with $\mathrm{BD}$, being $67 \%$ voluntary admitted and $33 \%$ involuntary. Data showed that there is SSD in the number of past decompensations, whether for depressive, maniac or hippomaniac episodes, as well as type of the current decompensation for VC patients. Additionally, there was a marginal SSD in the introduction of injectable treatment, as well as the age of $\mathrm{BD}$ diagnosis between those two populations. Nevertheless, we found no association or SSD in the other clinical and sociodemographic dimensions analyzed.

Conclusions: In general, compulsory hospitalizations create difficulties in the management, progress and response to treatment. Factors such as patient's vulnerability, defined by socio-demographic elements, clinical characteristics, social support, treatment adherence, have significant implications in prognosis, costs and care. The conducted study enabled us to outline a profile of voluntarily and involuntarily hospitalized and their differences.

Conflict of interest: No

Keywords: compulsory hospitalization; Bipolar disorder; voluntary hospitalisation
Bipolar disorders - Part II

\section{EPP0109}

\section{Gender differences among admitted patients with bipolar disorder during 2018}

F. Caldas ${ }^{1}$, P. Frias ${ }^{1}$, R. Valido ${ }^{1}$, M. Gonçalves ${ }^{2}$, D. Pires ${ }^{1}$, M. Barros ${ }^{1}$ and M. Falcão ${ }^{3}$

${ }^{1}$ Hospital de Magalhães Lemos, Psychiatry, Porto, Portugal; ${ }^{2}$ Hospital de Magalhães Lemos, Psychiatry, porto, Portugal and ${ }^{3}$ Centro

Hospitalar do Porto, Centro Materno Infantil Do Norte, porto,

Portugal

${ }^{\star}$ Corresponding author.

Introduction: Gender differences in Bipolar Disorder (BD) are becoming apparent but have been less studied compared with other disorders. Presentation, clinical features, course and evolution of BD differ between men and women. Research data on these differences will help determine whether gender is an important factor influencing BD.

Objectives: Retrospective and descriptive study to determine the influence of gender in socio-demographic, clinical and psychopathological variables of $\mathrm{BD}$.

Methods: Charts of BD patients admitted in Hospital de Magalhães Lemos (HML) during 2018 were reviewed. Statistical analysis of data.

Results: During 2018, 2890 patients were hospitalized in HML, 138 of which diagnosed with BD. The majority were female patients $(60 \%)$, married (41\%), retired (45\%) with ages between 20 and $82(\mathrm{M}=51,93)$ years old. Data showed statistically significant differences (SSD) in psychiatric comorbidity, such as Personality Disorder, Anxiety Disorder and Substance Use Disorder, whether present or past. Particularly, there are SSD in current alcohol and cannabis use. Also, men are more likely to smoke than women. The authors found marginal SSD in the length of hospitalization, when controlling for current episode type $(\mathrm{p}=.083)$. Men have longer hospitalizations $(M=47,88)$ than women $(M=22,56)$. Nevertheless, there was no association or SSD in other analyzed dimensions, such as bipolar subtype, age of onset, delay in diagnosis, number of episodes and suicide attempts, hospitalizations, family history and treatment options.

Conclusions: The importance of gender on course and outcome of $\mathrm{BD}$ has been acknowledged in the literature, suggesting that the prevalence is similar but the course of illness may be different.

Conflict of interest: No

Keywords: Bipolar disorder; Gender

Bipolar disorders - Part III

\section{EPP0123}

Metabolic disturbances as prognostic factors in bipolar disorder: a systematic review.

A. Giménez Palomo ${ }^{1}$, S. Dodd ${ }^{2}$, M. Berk ${ }^{2}$, S.P. Gomes ${ }^{1}$, M.T. Pons ${ }^{3}$, G. Anmella ${ }^{4}$, J. Pinzon Espinosa ${ }^{5}$ and I. Pacchiarotti ${ }^{6}$

${ }^{1}$ Hospital Clínic, Psychiatry, Barcelona, Spain; ${ }^{2}$ Barwon Health, Impact Src, Geelong, Australia; ${ }^{3}$ Hospital Clinic de Barcelona, Psychiatry, Barcelona, Spain; ${ }^{4}$ Hospital Clinic, University of Barcelona, IDIBAPS, 
CIBERSAM, Schizophrenia Unit, Institute Of Neuroscience, Barcelona, Spain; ${ }^{5}$ Hospital Clinic de Barcelona, Institut Clinic De Neurociencias, Barcelona, Spain and ${ }^{6}$ Bipolar and Depressive Disorders Unit, Hospital Clínic de Barcelona, Psychiatry, Barcelona, Spain ${ }^{\star}$ Corresponding author.

Introduction: There is evidence suggesting that individuals suffering from bipolar disorder (BD) are at increased risk for somatic diseases compared to healthy controls, such as obesity, hypertension, dyslipidemia, type 2 diabetes mellitus, and metabolic syndrome. Current literature suggests a strong relationship between metabolic disturbances and the course of BD. Nowadays, research pointing at potential implications of metabolic syndrome in worse clinical outcomes on BD is growing.

Objectives: We systematically reviewed the existing literature on the impacts of metabolic disturbances on the frequency of affective episodes, hospitalizations, cognitive impairment, suicidality and other potential outcomes on BD.

Methods: A careful search of articles on MEDLINE/PubMed and PsycINFO was conducted, including peer-reviewed articles from any time to August $5^{\text {th }}, 2019$. We selected articles assessing metabolic risk factors in the course of BD. We excluded studies not relating directly metabolic risk factors with $\mathrm{BD}$ outcomes, studies on the effects of metabolic disturbances without reporting results of each risk factor separately (apart from metabolic syndrome) and others not separating the bipolar group from other mental illnesses. Results: Thirty-six articles satisfied the selection criteria. Most evidence was focused on obesity as the main factor affecting the course of illness in BD, but others assessed blood lipid levels, metabolic syndrome and impaired glucose metabolism, showing inconsistent results.

Conclusions: Metabolic disturbances might contribute in the number of episodes, hospitalizations, suicide attempts, a chronic course of illness, cognitive impairment and poorer global functioning. Nevertheless, current evidence is controverted. Further research should elucidate the implications of each risk factor on the course of BD.

\section{Conflict of interest: No}

Keywords: Bipolar disorder; course of illness; prognosis; metabolic syndrome

\section{Bipolar disorders - Part V}

\section{EPP0145}

International convergences and divergences in metaanalyses of epidemiological studies of bipolar disorder

A.L. R. Moreira ${ }^{1 \star}$, E. Youngstrom ${ }^{2}$ and A. Van Meter $^{3}$

${ }^{1}$ Centro Hospitalar do Oeste, Departamento De Psiquiatria E Saúde Mental, Caldas da Rainha, Portugal; ${ }^{2}$ University of North Carolina at Chapel Hill, Department of Psychology and Neuroscience, Chapel Hill, NC, United States of America and ${ }^{3}$ The Donald and Barbara Zucker School of Medicine at Hofstra/Northwell, Department of Psychiatry, New York, United States of America

${ }^{\star}$ Corresponding author.

Introduction: Environmental and genetic factors that confer risk or protection for disease - including mental illness like bipolar disorder (BD) - are not uniformly distributed internationally.
Objectives: (1) To describe BD prevalence differences over time and across regions, and (2) to evaluate potential contributing factors.

Methods: Studies from a PubMed and PsycINFO search including the following terms: mania, bipolar, community, epidemiology, that included bipolar/mania prevalence rates were coded. Data were meta-analyzed using mixed models.

Results: There were 85 effect sizes from adult studies, dated 19802012 , from 44 countries, nearly one million people, 67,373 with BD. BDI prevalence was $0.62 \%$ and Spectrum $1.02 \%$. Rates are not changing over time; however, North and South America have rates two times higher than Africa and Asia. There were 19 effect sizes from pediatric studies, dated 1986-2017, from 12 countries, including 56,103 youths, 1383 with BD. BDI rate was $0.6 \%$ and Spectrum $3.9 \%$. Studies were heterogeneous; rates are not increasing over time, the use of broad criteria is associated with higher rates $(\mathrm{p}<.0001)$, as were older minimum age $(\mathrm{p}=.005)$, and lifetime prevalence $(\mathrm{p}=.002)$.

Conclusions: $\mathrm{BD}$ rates are not changing over time, but there are international differences with Asia and Africa having significantly lower rates. Pediatric BD is not more prevalent in the United States, but there is a lack of studies from non-western countries. Systematically evaluating $\mathrm{BD}$ prevalence internationally suggests risk for $\mathrm{BD}$ may grow internationally with globalization.

\section{Conflict of interest: No}

Keywords: epidemiology; community; Bipolar disorder; International

\section{Child and adolescent psychiatry - Part I}

\section{EPP0157}

\section{Autistic traits among Saudi university students; relations with emotional intelligence and alexithymia}

E.S. Soliman ${ }^{1}$, A. Almutairi ${ }^{2 \star}$, F. Almutairi ${ }^{2}$, H. Alswailem ${ }^{2}$, R. Salem ${ }^{2}$ and W. Albati ${ }^{2}$

${ }^{1}$ College of Medicine, Zagazig University, Psychiatry Department, Zagazig, Egypt and ${ }^{2}$ Princess Nourah Bint Abdulrahman University, College Of Medicine, Riyadh, Saudi Arabia

${ }^{*}$ Corresponding author.

Introduction: Autism spectrum disorder (ASD) is a developmental disability characterized by communication and social impairments. Alexithymia has been continuously associated with ASD due to deficiency in emotional component in both disorders. Measuring Emotional Intelligence (EI) in both disorders may reveal more insight about this relation.

Objectives: To determine the prevalence of ASD among Saudi university students and to explore the link between ASD and alexithymia focusing on EI.

Methods: We conducted a Cross-sectional study that included 600 students recruited via a convenient sampling technique. Data were collected using the following validated scales: Autism Spectrum Quotient (AQ10), Toronto Alexithymia Scale (TAS-20) and Trait Emotional Intelligence Questionnaire - Short Form (TEIQue-SF).

Results: revealed $7.7 \%$ prevalence of ASD, $34.8 \%$ possible Alexithymia and $42.2 \%$ true Alexithymia. Male students \& participants 
with a low GPA also were significantly associated with ASD (P = 0.032 and 0.012 , respectively). Females had higher emotional intelligence than males $(\mathrm{P}=0.022)$. Younger participants expressed a measurable link to emotional intelligence $(\mathrm{P}<0.05)$. Alexithymia score was significantly higher among females and among those below 21 years old $(\mathrm{P}=0.001)$. Approximately $16 \%$ of people with possible ASD also reported positive alexithymia. Total EI score showed no significant relation neither with ASD nor with Alexithymia.

Conclusions: Prevalence of ASD and Alexithymia were considerably high among Saudi University students. male gender and low GPA were significantly associated with ASD. Alexithymia was higher among females and younger participants. The association between total EI score and both ASD and Alexithymia was statically insignificant.

\section{Conflict of interest: No}

Keywords: Autism spectrum disease (ASD); Alexithymia; emotional intelligence; Saudi university students

\section{EPP0161}

Long acting injectable antipsychotics in children and adolescents with serious mental illness

M.D.P. Alvites Ahumada ${ }^{1 \star}$, E. Landa ${ }^{2}$, N. Serrano ${ }^{2}$, S. Guijarro ${ }^{2}$, M.D.C. Díaz Fernandez ${ }^{2}$ and M. Infante Sanchez De Lugarnuevo ${ }^{2}$

${ }^{1}$ Complejo Hospitalario Mancha Centro, Unidad De Salud Mental Infanto Juvenil, Alcázar de San Juan (Ciudad Real), Spain

${ }^{2}$ Hospital general de Tomelloso, Unidad De Salud Mental,

Tomelloso, Spain

${ }^{\star}$ Corresponding author.

Introduction: Children and adolescent are an special population. Althougth this type of medication is widely used in a aduls, there are few studies about the use of long acting injectable antipsychotics in this population.

Objectives: Educational objetives: Have a view of the treatment of children and adolescents with long acting antipsychotics in serious mental illness. Purpose: To make a theoretical review about the treatment of children and adolescents with a long acting antipsychotics in serious mental illness.

Methods: Case presentation and review of the literature.

Results: 16 year old male patient who began in Mental Health at 12 years old because difficulties to socialize with peers. He had an IQ of 71. He had the diagnosis of Autism Spectrum Disorder, and he started in a School for children with special needs. He always has been talking about death, accidents, murders, etc. He used to draw skulls, knifes, blood. Two years ago he started to say that a classmate insulted him. One day, with no provocation, he took a rake and assaulted him, causing a ulna fracture. In the inpatient Unit, he said that he insulted him, and he always used to did it. We began Aripiprazole in an ascending dose, then we switched to the long acting presentation. He is using this treatment during the last 20 months, and he is stable.

Conclusions: The literature review suggest long acting antipsichotics in children and adolescents with serious mental illness may improve clinical outcomes and adherence.

Conflict of interest: No

Keywords: long acting injectable antipsychotics; Children; adolescents; serious mental illness
Child and adolescent psychiatry - Part IV

\section{EPP0193}

From children pathology to psychosis. trauma and substancie use as risk marks.

E. Garcia ${ }^{\star}$, M. Garcia, P. Barredo, A. Sánchez Cabezudo and M. Soto

Sescam, Psychiatry, Toledo, Spain

${ }^{*}$ Corresponding author.

Introduction: Trauma is a well known independent factor of psychosis, however it has not been well discussed the rol of coping strategies between as a treatable target. ThisOral presentacion affords these two concepts and link them to clinical practise

Objectives: To determine how far we can achieve prevention as we treat trauma before psychosis may appear

Methods: We have a 300 adolescence patients with and without psychosis that have been stadistically studied, comparing coping strategies, trauma and clinical presentacion

Results: First, we have a wife range of stressors among our sample that may be alone, or combined. We selected a cualitative approach to analyse coping strategies, and linked them to biological and enviroment factors specially the ones refering to main caregiver. We observed obsesive and dissociative coping strategies were useful in focalized periods but not in a longitudinal way, as they become rigids patterns with low adaptability to Changes.

Conclusions: One target in our treatment has been recovery known as reaching asymptomathic condition. But when we observe patients in anlogitudinal way there are twokind of responses, short ones which take away symptoms and complex long ones which includes the management of stressors. We can only reach second onesof we worlk over coping patterns

Conflict of interest: No

Keywords: trauma; psychosis; Children; adolescence

\section{Child and adolescent psychiatry - Part V}

\section{EPP0207}

Acceptability and utility of psychoeducation and cognitive behavioural therapy for parents of children with functional neurological disorders: a pilot study

B. Baig ${ }^{1}$, H. Bhatt ${ }^{1}$, S. Gómez-Vallejo ${ }^{2 *}$, M. Cooney ${ }^{1}$ and N. Kuruppuarachchi ${ }^{1}$

${ }^{1}$ Kings College London, Child and Adolescent Psychiatry, Ioppn, London, United Kingdom and ${ }^{2}$ King's College London, Department of Child \& Adolescent Psychiatry, London, United Kingdom

${ }^{\star}$ Corresponding author.

Introduction: Functional Neurological Disorders (FND) are common and complex conditions in which medical explanatory models and treatment remain challenging. (1). Cognitive Behavioural Therapy (CBT) is an effective treatment for adults and group interventions in adolescents with anxiety and functional headaches 
are known to be effective. However, there is little literature regarding intervention with the families of children with FND.

Objectives: To analyse whether combined psychoeducational intervention and CBT for parents of children and adolescents with FND can reduce symptoms, increase function in children, manage parental distress through understanding their child's symptoms.

Methods: A workshop consisting of 8 sessions over 10 weeks will be delivered to 12 parents. The sessions will cover psychoeducation and CBT. Intrasubject differences in number of visits to Emergency Department (ED) or other health services, attendance to school, functioning and other measures such as anxiety will be assessed pre and post interventions for parents.

Results: The programme will be run twice over 6 month period. Results will be obtained from pre-post measures as well as therapy outcomes, qualitative interviews and child follow up measures including RCADS, SCARED, PedsQoL, family adjustment scale and Alexithymia scales.

Conclusions: We hypothesize that the workshop will reduce parents' isolation, increase understanding of diagnosis and will improve family engagement in the therapy. In addition, we also predict a decrease in the number of visits to the ED and the need of input from other health services. We hope this will add to treatment guidelines in children of FND.

\section{Conflict of interest: No}

Keywords: functional neurological disorders; Medically unexplained symptoms; family therapy; CBT

\section{Child and adolescent psychiatry - Part X}

\section{EPP0257}

"How to understand the development of identity and gender diversity in adolescents and young adults with autistic spectrum disorder (ASD)"

\section{T. Vandeusen}

Yale University School of Medicine, Psychiatry, New Haven, United States of America

Introduction: Recent literature has addressed the association between Autistic Spectrum Disorder (ASD) and Gender Dysphoria (GD) and or "gender diverse", a term used to describe those who identify as "non-binary", meaning somewhere on a continuum between male and female. Some studies have shown that 5-7\% of those with ASD have GD, compared to $1 \%$ in the general population, but others have wondered if those with ASD do not have GD but are simply "gender diverse".

Objectives: There are many unanswered questions pertaining to the association between the diagnoses of Autistic Spectrum Disorder (ASD) and Gender Dysphoria and/or Gender Diversity. This Oral Communication aims to shed light upon this topic offering clinical materials including a case vignette, to stimulate participant's questions, and provide sufficient time for a robust discussion. Methods: This presentation will provide a developmental perspective of ASD and illustrate the association of ASD and gender dysphoria/ gender diversity with a clinical case of a 23-year-old young adult.

Results: The presenter will give a broad overview of Autism through a developmental lens, focusing on the difficulty assessing the affirmed gender of the patient. He will describe a 23-year-old patient with a history of trauma, ASD, ADHD, and intellectual disability who stated he was a "girl because his mother dressed him that way". Conclusions: Psychiatrists are encouraged to use their developmental expertise to treat complex ASD patients and contemplate their patient's current cognitive state, social skill level, and cultural environment when making the diagnosis of Gender Dysphoria.

\section{Conflict of interest: No}

Keywords: child and adolescent development; gender identity; gender dysphoria; autistic spectrum disorder

\section{Classification of mental disorders}

\section{EPP0268}

\section{Social phobia and autism: two peas in a pod?}

F. Caetano ${ }^{1 \star}$, M. Araújo ${ }^{2}$, A. Samouco ${ }^{3}$, C. Priscila $^{1}$, P. Frias ${ }^{4}$ and S. Carvalho ${ }^{1}$

${ }^{1}$ Hospital de Magalhães Lemos, Serviço De Internamento B, Porto, Portugal; ${ }^{2}$ Hospital de Magalhães Lemos, Serviço De Internamento C, Porto, Portugal; ${ }^{3}$ Unidade Local de Saúde do Norte Alentejano, Department of Psychiatry and Mental Health, Portalegre, Portugal and ${ }^{4}$ Hospital de Magalhães Lemos, Psychiatry, Porto, Portugal ${ }^{*}$ Corresponding author.

Introduction: Autism Spectrum disorders, particularly highfunctioning autism (HFA), and Social Phobia (SP) can present with similar behavior patterns and sometimes be comorbid. In adults, ASD and SP may become increasingly more difficult to distinguish as maturation may lead to reduced symptom severity or more subtle deficits.

Objectives: Review the existing literature on HFA and SP and outline similarities, overlaps and differences to aid diagnosis.

Methods: Literature search on Pubmed and Google Scholar

Results: Both HFA and SP involve profound social interaction deficits. Although these disorders share some similar symptoms, they are conceptualized as distinct. To distinguish these two entities clinicians must rely on behavioral assessment. Major domains across which HFA and SP appear similar but which in fact differ in subtle ways include: eye contact; stereotypical nonverbal behaviors; beliefs; relating and communicating in a social context; interpreting social cues, reciprocal interaction, friendship patterns and others. Psychiatric comorbidities are common in HFA. Recent research suggests that these patients may exhibit significantly higher levels of anxiety than the general population with high rates of SP.

Conclusions: Due to overlapping of symptoms, diagnosis of HFA and/or SP can be challenging. Better understanding of similarities and differences can help clinicians to accurately diagnose these patients and to offer them better treatment.

Conflict of interest: No

Keywords: SOCIAL PHOBIA; Autism

\section{EPP0272}

\section{Major depression as an expression of frontal lobe dysfunction}

R. Valido*, F. Caldas, M. Barros and P. Ferreira 
Hospital de Magalhães Lemos, Psychiatry, Porto, Portugal

${ }^{*}$ Corresponding author.

Introduction: Frontal systems dysfunction can present clinically very heterogeneously. Frontal subcortical circuits are very important in a myriad of psychiatric conditions including depressive disorders. Objectives: We aim to establish the importance of the differential diagnosis between Frontal Lobe Syndromes, Affective Disorders, and Dementia resorting to a clinical case report.

Methods: Clinical case report and a brief review of the literature. Results: A 55-year-old caucasian male was admitted to the inpatient unit after developing depressive symptoms and suicidal attempts in the months following a striatal lacunar stroke. The patient had an MMSE of 26/30 and had criteria for a Major Depressive Episode according do DSM-5. However, he punctuated 13/30 in INECO Frontal Screening, a screening test for Executive Cognitive Functions, with a working memory index of 4/10. He also punctuated 6/12 in Apathy Inventory. We assumed the patient had a subcortical dementia and a prefrontal syndrome - dysexecutive type, mediated by the dorsolateral prefrontal lobe and its connections. It is characterized by diminished judgment, planning, insight and temporal organization. The neuropsychological evaluation revealed impaired attention and concentration, difficulty with registration and retrieval, and decreased verbal fluency. Additionally, cognitive impersistence and diminished self-care were present. The patient enrolled in a cognitive rehabilitation program and was medicated with an SSRI and quetiapine with good results.

Conclusions: Affective symptoms may occur subsequently to a dysexecutive syndrome rather than primary affective disorders or dementia syndromes. Prefrontal cortico-subcortical dysfunction originates altered emotional and motivated behavior processing. Clinicians need to be aware of this possible explanation for depressive-like symptoms.

Conflict of interest: No

Keywords: Frontal Lobe; Prefrontal syndrom; Major Depressive Episode; Executive Cognitive Functions

\section{Comorbidity / dual pathologies - Part I}

\section{EPP0281}

\section{Dual pathology and gender differences}

F. Caldas ${ }^{1 \star}$, P. Frias ${ }^{2}$, R. Valido ${ }^{2}$, M. Barros ${ }^{2}$, D. Pires ${ }^{2}$, M. Gonçalves ${ }^{1}$ and M. Falcão ${ }^{3}$

${ }^{1}$ Hospital de Magalhães Lemos, Psychiatry, porto, Portugal; ${ }^{2}$ Hospital de Magalhães Lemos, Psychiatry, Porto, Portugal and ${ }^{3}$ Centro Hospitalar do Porto, Centro Materno Infantil Do Norte, porto, Portugal

${ }^{*}$ Corresponding author.

Introduction: According to the National Survey on Drug Use and Health of 2017, the estimated prevalence of Dual Pathology (DP) is about $19 \%$, of which $4,5 \%$ severe. Psychopathology is undoubtedly a risk factor for additive disturbance, and vice versa, both associated with worse prognosis.

Objectives: A retrospective and descriptive study of the patients with DP hospitalized in Hospital Magalhães Lemos (HML) during 2018, analyzing several sociodemographic and clinical dimensions. Methods: Consulting clinical files and statistical analyzation of the data.
Results: During 2018, 2890 individuals were hospitalized at HML, of which 290 with PD (78\% males, $22 \%$ females). The high level of professional inactivity (47\%) and the large number of unmarried individuals $(48.6 \%)$ stand out in both genders. The age pattern found was considerably different (25 years for men and 51 for women) but both were more frequently voluntarily hospitalized. Men predominantly consume alcohol (60\%) and cannabis $(52.4 \%)$, while this sequence is reversed in females. There was also a slight difference in the most frequent psychiatric disorder, with women having a higher frequency of personality disorders (26.2\%) compared to schizophrenia (16.9\%), unlike men in that the proportion is similar ( $20 \%$ for both). Furthermore, statistically significant (SS) differences were found between gender and length of hospital stay and injectable treatment (before and after hospitalization), as well as SS associations between gender and inpatient regime.

Conclusions: These data reinforce the need to characterize the profile of these patients, anticipating difficulties and outcomes and understanding their needs in order to adequate prevention and reintegration.

Conflict of interest: No

Keywords: Gender; dual pathology

\section{Comorbidity / dual pathologies - Part II}

\section{EPP0291}

Interictal dysphoric disorder in patients with and without epilepsy

G. Kustov ${ }^{1 \star}$, F. Rider ${ }^{2}$, E. Pashnin ${ }^{3}$, K. Pochigaeva ${ }^{4}$, A. Yakovlev ${ }^{3}$ and M. Zinchuk ${ }^{5}$

${ }^{1}$ Moscow Research and Clinical Center for Neuropsychiatry, Department of New Methods Of Therapy Of Mental Disorders, Moscow, Russian Federation; ${ }^{2}$ Moscow Research and Clinical Center for Neuropsychiatry, Department of Epidemiology- Prophylaxis and Healthcare, Moscow, Russian Federation; ${ }^{3}$ Moscow Research and Clinical Center for Neuropsychiatry, Suicidology (crisis), Moscow, Russian Federation; ${ }^{4}$ Moscow Research and Clinical Center for Neuropsychiatry, Department of Nonpsychotic Mental Disorders In Neurologic and Geriatric Patients, Moscow, Russian Federation and ${ }^{5}$ Moscow research and clinical center for neuropsychiatry, Suicidology (crisis), Moscow, Russian Federation

${ }^{*}$ Corresponding author.

Introduction: Interictal dysphoric disorder (IDD) considered to be an affective disorder which occurs only in patients with epilepsy (PWE). Controversial data of the association of IDD with epilepsy characteristics and equal prevalence of IDD in PWE and patients with migraine or psychogenic non-epileptic seizures challenged its epilepsy-specific nature.

Objectives: To investigate epilepsy-specific nature of IDD

Methods: 142 consecutive hospitalized PWE and major depressive disorder (MDD) confirmed by structured clinical interview (SCID) for DSM-IV were included at the main group. The comparative group consisted of 222 inpatients with MDD alone. The groups were comparable by age and gender. We used IDD inventory to confirm the presence of IDD. Mann-Whitney, ANOVA, Pearson's chi-squared and Spearman's correlation were used as statistical methods 
Results: We didn't find any significant differences in prevalence of IDD between PWE and depression and patients with MDD alone ( $88.73 \%$ vs $85.13 \%: \chi^{2}=0.96, p=0.32$ ). There were no differences between groups regarding the mean number of all IDD symptoms and each symptom cluster (all $\mathrm{p}>0.05$ ). In both groups symptoms of IDD were clustered with the same frequency $\left(\chi^{2}=2.22 ; \mathrm{p}=0.13\right)$ and had an equal duration $\left(\chi^{2}=10.5 ; \mathrm{p}=0.06\right)$ and periodicity $\left(\chi^{2}=3.22\right.$; $\mathrm{p}=0.12)$. PWE with $(\mathrm{N}=126)$ and without IDD $(\mathrm{N}=16)$ didn't differed in terms of epilepsy type $\left(\chi^{2}=3.05 ; \mathrm{p}=0.08\right)$, duration $(p=0.86)$, age of onset $(p=0.49)$, seizure frequency $\left(\chi^{2}=1.81\right.$; $\mathrm{p}=0.4)$; epileptogenic zone localization $\left(\chi^{2}=5.71 ; \mathrm{p}=0.22\right)$ and number of antiepileptic drugs taken $\left(\chi^{2}=0.74 ; \mathrm{p}=0.68\right)$. There were no significant associations between epilepsy characteristics and total number of IDD symptoms (all p>0.05).

Conclusions: Our results couldn't confirm epilepsy-specific nature of IDD.

\section{Conflict of interest: No}

Keywords: Interictal dysphoric disorder; major depressive disorder; Dépression; Epilepsy

\section{EPP0292}

\section{Comorbidity of bipolar affective disorder and epilepsy}

G. Kustov $^{1 \star}$, E. Pashnin ${ }^{2}$, M. Zinchuk ${ }^{3}$ and R. Akzhigitov ${ }^{4}$

${ }^{1}$ Moscow Research and Clinical Center for Neuropsychiatry, Department of New Methods Of Therapy Of Mental Disorders, Moscow, Russian Federation; ${ }^{2}$ Moscow Research and Clinical Center for Neuropsychiatry, Suicidology (crisis), Moscow, Russian Federation; ${ }^{3}$ Moscow research and clinical center for neuropsychiatry, Suicidology (crisis), Moscow, Russian Federation and ${ }^{4}$ Moscow Research and Clinical Center for Neuropsychiatry, Deputy Director, Moscow, Russian Federation

${ }^{\star}$ Corresponding author.

Introduction: Patients with epilepsy (PWE) are at higher risk for active depression compared to general population. It's speculated that epilepsy and bipolar disorder (BD) share common pathophysiology due to their episodic and chronic course, efficacy of several antiepileptic drugs in treatment and so on. Studies regarding the prevalence of BD in PWE are limited and their results are controversial.

Objectives: To investigate the prevalence of $\mathrm{BD}$ in PWE and current major depressive episode (MDE).

Methods: 150 consecutive hospitalized PWE and current MDE were compared with 300 hospitalized patients with MDE episode alone. All patients were assessed with structured clinical interview (SCID) for DSM-IV to verify the presence of MDE and the lifetime history of mania/hypomania. PWE with manic/hypomanic symptoms associated with seizure activity or antiepileptic drug (AED) treatment were excluded from the analysis. The groups were comparable by age, gender and the duration of affective disorder. MannWhitney, Pearson's chi-squared and logistic regression were used as statistical methods.

Results: The prevalence of lifetime mania/hypomania in depressed PWE was $5.3 \%(\mathrm{~N}=8)$ compared with $26 \%(\mathrm{~N}=78)$ of those without epilepsy $(\chi 2=27.629, \mathrm{p}<0.001)$. The odds ratio of subjects with MDE alone having $\mathrm{BD}$ relative to those with epilepsy was $6.23(95 \%$ $\mathrm{CI}=2.92$-13.02: $\mathrm{p}<0.001)$. There were no significant associations between BD, epilepsy characteristics and AED with moodstabilizing properties treatment in PWE (all p>0.05).

Conclusions: PWE and MDE appeared less likely to have BD than those without epilepsy, but the causes of this discrepancy still have to be clarified.

Conflict of interest: No

Keywords: Epilepsy; Bipolar disorder; Bipolar depression

\section{Comorbidity / dual pathologies - Part IV}

\section{EPP0310}

\section{"Drink to forget" - a case report}

M. Lázaro ${ }^{1}$, L. Gil ${ }^{2}$, S. Ferreira ${ }^{3}$, P. Diegues ${ }^{3}$, J. Teixeira ${ }^{3 \star}$ and T. Mota $^{3}$

${ }^{1}$ Centro Hospitalar Psiquiátrico de Lisboa, Clínica 5, Lisboa, Portugal; ${ }^{2}$ Centro Hospitalar Psiquiátrico de Lisboa, Clínica 6, Lisboa, Portugal and ${ }^{3}$ Centro Hospitalar Psiquiátrico de Lisboa, Serviço Alcoologia E Novas Dependências, Lisboa, Portugal

${ }^{*}$ Corresponding author.

Introduction: Attention, working memory, speed of processing, visuospatial abilities, executive functions, impulsivity, learning, memory and verbal fluency are cognitive functions that can be impaired in alcoholism. These cognitive impairments are underestimated in clinical practice, even though they could limit alcohol treatment.

Objectives: Theme review on the available evidence regarding alcohol-related cognitive impairment. Presentation of a case report concerning a patient with alcohol dependence syndrome and cognitive complaints.

Methods: Non-systematic review of the literature was performed in PubMed/Medscape database. A patient's clinical record was reviewed.

Results: We present a case report of a 44-year-old male with alcohol dependence and comorbid anxiety disorder, who complains of memory and impulse control problems. The patient has alcohol consumption since 21 years old, with gradual increase. He drinks mainly beer and spirits drinks. He was admitted to inpatient care and after discharge, started the relapse prevention intervention. After 5 months of alcohol abstinence he maintained memory and impulse control problems. The patient was evaluated and some screening tools were applied (Montreal Cognitive Assessment, Addenbrooke Cognitive Examination and Frontal Assessment Battery). In this patient, verbal fluency, memory, attention, visuospatial capacities and impulsivity were impaired. The patient was included in a Neuromodulation Cognitive Program at Centro Hospitalar Psiquiátrico de Lisboa.

Conclusions: This case report highlights the neurocognitive effect of excessive and long-term consumption of alcohol, bringing a better understanding of alcohol-related cognitive disorders usually hidden by confounding factors. It is essential to take into account the cognitive dimension of alcohol-dependent patients in order to adapt their treatment.

\section{Conflict of interest: No}

Keywords: Alcohol dependence; Cognitive impairment; Anxiety; relapse 
Consultation liaison psychiatry and psychosomatics Part I

\section{EPP0323}

\section{CPAP-induced mania in a man with obstructive sleep apnea}

D. Esteves-Sousa ${ }^{1 \star}$, J. Facucho-Oliveira ${ }^{2}$, M. Albuquerque ${ }^{1}$, N. Moura ${ }^{3}$, P. Espada-Santos ${ }^{1}$ and L. Mendonça ${ }^{1}$

${ }^{1}$ Hospital de Cascais Dr. José de Almeida, Departamento De Psiquiatria E Saúde Mental, Alcabideche, Portugal; ${ }^{2}$ Hospital de Cascais, Department of Psychiatry, Cascais, Portugal and ${ }^{3}$ Centro Hospitalar Lisboa Ocidental, Department of Psychiatry, Lisboa, Portugal ${ }^{\star}$ Corresponding author.

Introduction: A connection between sleep disturbances and psychiatric disorders has been reported in the literature. Obstructive sleep apnea OSA has been systematically associated with depressive disorders. A recent large cohort study also revealed an association with anxiety disorders, post-traumatic stress disorder, psychosis and bipolar disorders. Additionally, there's a couple of case-reports of manic episodes associated with CPAP initiation.

Objectives: Our aim is to perform a systematic review of the literature about the association between OSA, continuous positive airway pressure (CPAP) and bipolar disorder. Additionally, we present a case-report about an onset of mania after CPAP in a patient with OSA.

Methods: Systematic review of the literature in scientific databases Google Scholar and Pubmed. The case-report was described with data retrieved from the clinical file.

Results: Here, we report, a case of a 53-year-old man with no personal or family history of psychiatric illness, with a severe OSA. Patient initiated CPAP and developed manic symptoms about one month after treatment. An underlying medical cause was ruled out. The psychiatry symptoms reverted upon cessation of CPAP. Two further manic episodes were observed with re-initiation of CPAP, the last one already with mood stabilizers and antipsychotics.

Conclusions: This case shows a OSA patient that displayed a first manic episode upon treatment with CPAP and two further manic episodes as CPAP was reinitiated. This adds present evidence to the previously raised hypothesis of a possible association.

Conflict of interest: No

Keywords: CPAP; Bipolar disorder; OBSTRUCTIVE SLEEP APNEA; mania

\section{Depressive disorders - Part I}

\section{EPP0369}

Examining the effectiveness of a culturally appropriate intervention in reducing postpartum depression among bedouin women in southern israel

S. Alfayumi-Zeadna

Ben-Gurion University, Public Health, Lehavem, Israel
Introduction: Postpartum depression (PPD) is a common mental health problem among mothers worldwide and is more frequent among minority mothers, who face multiple barriers in accessing and utilizing mental health services for PPD prevention and treatment.

Objectives: This study sought to develop and evaluate the effectiveness of a culturally appropriate intervention (CAI) in reducing PPD among Bedouin women in southern Israel.

Methods: A quasi-experimental intervention included 332 women, with 169 of the women participating in the intervention, and 163 acting as controls. The intervention group received social support, individual consultation on PPD treatment and group lectures on PPD symptoms and other women's health issues during pregnancy and after birth. The staff at the intervention clinic received a training on PPD We compared PPD symptom differences between the study groups pre- and post-intervention. Participants were interviewed before the intervention, at 26 to 38 weeks of pregnancy and 2 to 4 months postpartum.

Results: The CAI intervention was effective in reducing PPD symptoms, with a greater decrease seen in the intervention group (from $38.5 \%$ to $17.2 \%, \mathrm{PV}<0.001$ ) compared to the control group (31.9\% and 29.4\%, PV=0.664, respectively). The odds ratio of having PPD was 2.6 times higher among participants than in the control group. These PPD differences between study groups remained significant even after adjustment for different factors.

Conclusions: Our study sheds light on the impact of CAI on the reduction of PPD among ethnic minority mothers. CAI to reduce PPD can overcome barriers to PPD prevention and treatment and lead to a significant reduction of PPD.

\section{Conflict of interest: No}

Keywords: postpartum depression; Bedouin women in Israel; culturally appropriate intervention

\section{Depressive disorders - Part II}

\section{EPP0385}

Transcranial direct-current stimulation (tDCS) as an adjuvant therapy for treatment-resistant depression (TRD)

J. Facucho-Oliveira ${ }^{1 \star}$, D. Esteves-Sousa ${ }^{1}$, P. Espada-Santos ${ }^{1}$, M. Albuquerque ${ }^{2}$, M. Costa ${ }^{2}$, L. Mendonça ${ }^{2}$ and R. Neves ${ }^{3}$

${ }^{1}$ Hospital de Cascais, Department of Psychiatry, Cascais, Portugal; ${ }^{2}$ Hospital de Cascais Dr. José de Almeida, Departamento De Psiquiatria E Saúde Mental, Alcabideche, Portugal and ${ }^{3}$ Casa de Saúde da Idanha, Irmãs Hospitaleiras do Sagrado Coração de Jesus, Department of Psychiatry, Lisboa, Portugal

${ }^{\star}$ Corresponding author.

Introduction: Treatment-resistant depression (TRD) is a highly debilitating disease associated with a massive socioeconomic burden. Despite, electroconvulsive therapy (ECT) being well known for its therapeutic effects, a subset of these patients fail to attain remission or require maintenance ECT at an unfeasible frequency. Recent evidence points transcranial direct-current stimulation (tDCS) as an alternative treatment for TRD.

Objectives: Scrutinize the relevance of tDCS in TRD patients. 
Methods: Here, we report a case study, selected from a consecutive case-series study, of patient with a highly debilitating TRD that was treated with tDCS.

Results: A 45 years-old man, with a psychiatric history with 2 major depressive episodes, developed depressive mood, anhedonia and apathy leading to social isolation and inability to work (HAMD score of 33). Various trials of antidepressant were carried out but no significant clinical benefit was observed, and the patient further developed sense of hopelessness with suicidal ideation. ECT treatment resulted in temporary improvement of depressive symptoms but the patient experienced a full relapse just one week after the completion of the treatment. An attempt with tDCTs was then initiated and the patient experienced a gradual clinical improvement attaining complete symptomatic remission after 8 weeks (HAMD score of 6). Currently, the patient has endured 10 months of treatment with $\mathrm{tDCS}$ and Vortioxetine with no signs of relapse or evidence of side effects.

Conclusions: tDCS appears to be an efficient therapy for TRD patients and be should be considered upon exhaustion. We are currently following this approach in order to assess the reliability of our initial evidence.

Conflict of interest: No

Keywords: tDCS; Depression; TRD

\section{EPP0390}

Improvement in depressive symptoms and various functional aspects after treatment of major depression with vortioxetine. Interim results from the "real-life effectiveness of vortioxetine" (relieve) study

P.-M. Llorca ${ }^{1}$, R. Jain ${ }^{2}$, M. Touya ${ }^{3}$, K. Simonsen ${ }^{4}$ and L. HammerHelmich $^{5 *}$

${ }^{1}$ University Clermont Auvergne, Department of Psychiatry, Clermont Ferrand, France; ${ }^{2}$ Texas Tech University School of Medicine, Department of Psychiatry, Lubbock, United States of America; ${ }^{3}$ Lundbeck, Health Economics and Outcomes Research, Deerfield, United States of America; ${ }^{4} \mathrm{H}$. Lundbeck A/S, Health Economic and Epidemiology Statistics, Valby, Denmark and ${ }^{5} \mathrm{H}$. Lundbeck A/S, Medical Affairs Value Evidence, Valby, Denmark

${ }^{\star}$ Corresponding author.

Introduction: Clinical trials demonstrated vortioxetine as an efficacious, well-tolerated antidepressant. However, determination of real-world outcomes is needed.

Objectives: To assess real-life effectiveness of vortioxetine as routine treatment for MDD on functioning related to work/school, social life and family/home responsibilities.

Methods: RELIEVE is a non-interventional, multi-national, prospective cohort study of outpatients initiating vortioxetine treatment for MDD as routine care and followed for 6 months. Here we present interim results of the first 358 patients followed 3 months. Functioning was measured by Sheehan Disability Scale (SDS) total and sub-scores. Depressive symptoms were measured by Patient Health Questionnaire 9-item (PHQ-9). Baseline characteristics were summarised descriptively, mixed models assessed improvements between baseline and month 3 , adjusted for relevant confounders.

Results: Of the 358 patients, 26 were from Canada, 71 from France, 145 from Italy and 116 from the United States. Majority were women (65.6\%), mean age (SD) was 50.4 (14.9) years. At baseline, mean (SD) PHQ-9 score was 17.8 (6.4) indicating moderately severe depression. Mean (SD) SDS total score was 19.0 (6.7) and mean (SD) sub-scores for disruption of work/school; social life and family life/home responsibilities were 6.5 (2.6); 6.6 (2.3) and 6.7 (2.4) respectively, indicating marked functional impairment. Confounder-adjusted mean (SE) changes from baseline were -5.63 (0.35) in PHQ-9 score; -6.51 (0.43) in SDS total score; -2.16 $(0.19) ;-2.14(0.16)$ and $-2.23(0.16)$ in the sub-scores respectively, all indicating statistically significant improvements from baseline to month 3 .

Conclusions: Patients with MDD treated with vortioxetine as routine care experienced improved functioning overall and on all sub-scores within 3 months.

Disclosure: I am a full time employee of $\mathrm{H}$. Lundbeck A/S

Keywords: vortioxetine; Real World Evidence; functional impairment; Depressive symptoms

Eating disorders - Part I

\section{EPP0441}

Anorexia and autism: which spectrum are we speaking about?

T. Duarte ${ }^{1 *}$, F. Fernandes Órfão ${ }^{2}$, C. Cordeiro $^{1}$, I. Duarte Silva ${ }^{1}$, B. Côrte-Real ${ }^{1}$, G. Andrade ${ }^{1}$ and A. Neves ${ }^{1}$

${ }^{1}$ Centro Hospitalar Universitário Lisboa Norte, Serviço De Psiquiatria E Saúde Mental, Lisboa, Portugal and ${ }^{2}$ ACES Almada Seixal, Usf Cuidar Saúde, Seixal, Portugal

${ }^{*}$ Corresponding author.

Introduction: Anorexia nervosa (AN) and autism spectrum disorder (ASD) form a relatively common comorbidity, with up to one in four individuals with anorexia nervosa (AN) being on the autistic spectrum. Unfortunately, these autistic traits may not have been recognized or diagnosed prior to eating disorder (ED) treatment.

Objectives: Non-systematic literature review on the relationship between AN and ASD.

Methods: PubMed database was searched between 2011 and 2019and articles with the words "anorexia nervosa", "autism", "autism spectrum disorders", "female ASD" and "eating disorders" were included.

Results: There is a higher prevalence of diagnosed ASD in individuals with AN compared to the healthy population. This relationship between the two conditions appears to be underpinned by common underlying neuropsychological and social problems, with individuals with AN exhibiting neurocognitive problems more typically associated with ASD. AN has been associated with significant inefficiencies in theory of mind, cognitive flexibility and central coherence. Individuals with $\mathrm{AN}$ are also characterized by poor social and emotional functioning, including interpersonal problems, impaired facial emotion recognition, diminished facial emotion expression, and social anhedonia. These problems are associated with a longer illness duration, higher illness severity, and poor treatment outcomes. These cognitive, socio-emotional and interpersonal problems in fact act as maintaining factors for the disorder itself. It is therefore possible that the presence of underlying ASD traits effectively maintains AN. 
Conclusions: Co-morbid AN and ASD may require more intensive treatment or specifically tailored interventions. Future longitudinal research and female-specific diagnostic tools would help elucidate the relationship between these two disorders.

Conflict of interest: No

Keywords: Anorexia nervosa; autism spectrum disorders; eating disorders; female ASD

\section{Emergency psychiatry - Part I}

\section{EPP0508}

Practical experiences with agitation in psychiatric emergencies (practice): design and rationale

K. Kahl ${ }^{1 \star}$, T. Messer ${ }^{2}$, S. Bradl ${ }^{2}$ and J. Kuhn ${ }^{3}$

${ }^{1}$ Hannover Medical School, Psychiatry, Social Psychiatry and Psychotherapy, Hannover, Germany; ${ }^{2}$ Danuvius Klinik Pfaffenhofen, Psychiatry, Pfaffenhofen a.d. Ilm, Germany and ${ }^{3}$ Evangelisches Krankenhaus Niederrhein, Psychiatrie, Psychotherapie Und Psychosomatik, Oberhausen, Germany

${ }^{*}$ Corresponding author.

Introduction: Psychiatric emergencies belong to the most difficult and clinically challenging situations in psychiatry. The etiology of psychiatric emergency conditions is typically multifaceted. Clinicians often face the problem that diagnostic evaluation is difficult due to the patients condition, and therefore treatment recommendations are often preliminary and syndrome oriented. Interestingly, studies examining clinicians real life diagnostic assessment and treatment recommendations in emergency situations are sparse. Therefore, PRACTIcE aimed at assessing clinicians decisions and patients satisfation in emergency situations in different clinical settings.

Objectives: To determine whether psychopharmacological treatment of agitation in psychiatric emergencies is performed according to current treatment recommendations. To determine whether patients satisfactory with emergency treatment changes after "agitation treatment training (ATT)" in psychiatrists.

Methods: Experimental intervention: Agitation treatment training (ATT) based on current treatment recommendations . All participating clinics are observed 3 months before and 3 months after the specific ATT.

Results: of the first assessment are complete, and physicians in participating centers were trained in ATT.

Conclusions: Ineffective management of acute emergencies and agitation can result in unnecessary use of coercive measures, including manual or mechanical restraint. Training clinicans in psychiatry is important when trying to reduce restrictive practices. Recognising the early signs of agitation is crucial, with the aim of reducing further restrictive interventions, and staff should be trained in the use of techniques aimed at defusing anger. Evaluation of newer concepts such as ATT gives the opportunity to improve clinical emergency management, and to include the patients perspective.

Disclosure: The study was supported by an unrestricted grant by Trommsdorf.

Keywords: Agitation; PATIENT SATISFACTION; emergency treatment
Epidemiology and Social Psychiatry - Part I

\section{EPP0517}

Socio-demographic characterization of the population accompanied by the mental health home-based care team at the vale do sousa region

R. Barranha ${ }^{\star}$, D. Gomes, J.P. Ribeiro and O. Von Doellinger

Centro Hospitalar do Tâmega e Sousa, Department of Psychiatry and Mental Health, Penafiel, Portugal

${ }^{\star}$ Corresponding author.

Introduction: The Department of Psychiatry and Mental Health of Centro Hospitalar do Tâmega e Sousa provides specialized mental health care to a population of about 520,000 people. Our Department offers a home-based care team that aims to promote the therapeutic compliance of patients with major psychiatric disorders, particularly through home visits, monitoring and administrating long acting antipsychotics. At the end of 2018, this service integrated a total of 286 patients.

Objectives: To evaluate the degree of social and family support provided to patients with major psychiatric disorders in the Vale do Sousa region, and characterize their financial situation.

Methods: The data were collected through a structured interview, conducted during home visits throughout October and December of 2018, gathering demographic data, professional situation and monthly income, household and living conditions, personal and family psychiatric history, medical comorbidities and monthly expenses directly related to medication. From clinical records were extracted the psychiatric diagnosis and the antipsychotic administered. This investigation was approved by the hospital ethics committee.

Results: We collected data from 119 patients (41,6\% of total). From the results disclosed we can summarize that the vast majority of our patients is diagnosed with Paranoid Schizophrenia, is retired, and earning a monthly disability pension of less than 300 euros. Furthermore, a significant percentage lives with their parents or with relatives, often sharing (or being excused from) house expenses.

Conclusions: Severe mental illness is an important cause of loss of work functionality and social exclusion. Patients in this Portuguese region are mainly dependent on their families' support.

\section{Conflict of interest: No}

Keywords: Home-base care team; Severe mental illness; Social support; Socio-demographic characterization

\section{EPP0520}

Prescribing patterns of anxiolytic medication among physician specialties in the slovak republic in 2014-2017

A. Brazinova ${ }^{1 \star}$, V. Sivcova ${ }^{1}$ and D. Breznoscakova ${ }^{2}$

${ }^{1}$ Faculty of Medicine, Comenius University, Institute Of Epidemiology, Bratislava, Slovak Republic and ${ }^{2}$ PJ Safarik University, Department of Psychiatry, Kosice, Slovak Republic

${ }^{*}$ Corresponding author.

Introduction: Monitoring of prescription and consumption of psychopharmacological treatment is an important part of the use of mental health care services evaluation. As the assessment of such prescription according to the specialty of prescribing physician has 
not been conducted in the Slovak Republic yet, we have carried out a study of the use of anxiolytics in the country in 2014-2017.

Objectives: The objective of the study is to evaluate the patterns of anxiolytics prescription in the Slovak Republic in 2014-2017 by age and sex of the patients and by the specialty of the prescribing physicians.

Methods: We obtained the data from the database of healthcare claims. We extracted the numbers of individual patients that had any of the ATC groups N05, N06 and N07 prescribed. For the study we used the information on the prescription of benzodiazepine and non-benzodiazepine anxiolytics prescribed by patient age and sex and by the prescribing physician specialty.

Results: In the study period $9.4 \%$ of the population used anxiolytics in the Slovak Republic, more women (11.8\%) than men (6.9\%). Consumption increased with age, the highest was in men 90 years and older (52\%). Benzodiazepine anxiolytics are prescribed more often than non-benzodiazepine (5.7 vs.3.7\%). Benzodiazepine anxiolytics are prescribed mainly by general practicioners (GP), non-benzodiazepine up to the age of 35 mainly by psychiatrists, after that age mainly by the GP.

Conclusions: The monitoring of the psychopharmacological treatment prescription in a population allows for guidance on medication consumption and standard therapeutic measures.

Conflict of interest: No

Keywords: anxiolytic; médication; prescription; Psychiatric

\section{Forensic psychiatry - Part I}

\section{EPP0564}

Involuntarily admitted patients in a psychiatric hospital during 2018

F. Caldas ${ }^{1 \star}$, P. Frias ${ }^{1}$, R. Valido ${ }^{1}$, M. Gonçalves ${ }^{2}$, D. Pires ${ }^{1}$, M. Barros ${ }^{1}$ and M. Falcão ${ }^{3}$

${ }^{1}$ Hospital de Magalhães Lemos, Psychiatry, Porto, Portugal; ${ }^{2}$ Hospital de Magalhães Lemos, Psychiatry, porto, Portugal and ${ }^{3}$ Centro

Hospitalar do Porto, Centro Materno Infantil Do Norte, porto,

Portugal

${ }^{\star}$ Corresponding author.

Introduction: Compulsory admission is the last line of intervention in individuals who suffer from severe mental disorders and refuse treatment, based on the principles of therapeutic need and social protection. In Portugal, the last law regulating the compulsory admission is in forme since 1999 (Law 36/98 of 24 July), and configures this measure as a hospitalization by court order, happening the same in other European countries.

Objectives: To outline the profile of involuntarily admitted (IA) patients, assessing socio-demographic and multiple clinical characteristics.

Methods: Consultation of clinical files of patients IA in Hospital Magalhães Lemos during 2018. Statistical analysis of data with "SPSS 21".

Results: During 2018, a total of 2890 patients were hospitalized and about one third of these were IA. The majority were male patients. The authors characterized socio-demographic data such as age, level of education, marital and employment status and social support. Regarding clinical data, authors analyzed psychiatric diagnosis, comorbidities, suicide attempts, number of hospitalizations and length, course of disease, number of patients previously followed in psychiatric consultation, as well as the percentage of first compulsorily psychiatric hospitalization, type of medication, treatment adherence and outcome.

Conclusions: In general, IA patients create difficulties in the management, progress and response to treatment. Factors such as patient's previous vulnerability, type and severity of their mental illness and social support have significant implications on prognosis and health burden. The conducted study enabled us to outline a profile of IA patients, allowing physicians to reflect on therapeutic options and guidance after discharge in order to provide better care.

Conflict of interest: No

Keywords: involuntary admittion; serious mental illness

Genetics \& Molecular neurobiology

\section{EPP0585}

\section{No association between akt1 gene variants and} schizophrenia in the russian population

E. Poltavskaya $^{1 \star}$, E. Kornetova $^{2}$, A. Boiko ${ }^{3}$ and O. Fedorenko ${ }^{1}$

${ }^{1}$ Tomsk National Research Medical Center of the Russian Academy of Sciences, Mental Health Research Institute, Tomsk, Russian Federation; ${ }^{2}$ Mental Health Research Institute, Tomsk National Research Medical Center, Russian Academy of Sciences, Department of Endogenous Disorders, Tomsk, Russian Federation and ${ }^{3}$ Mental Health Research Institute, Tomsk National Research Medical Center of the Russian Academy of Sciences, Laboratory Of Molecular Genetics and Biochemistry, Tomsk, Russian Federation ${ }^{*}$ Corresponding author.

Introduction: The dopamine hypothesis is the longest standing pathoetiologic theory of schizophrenia. AKT1 take part in one of the intracellular cascades activated by the D2 dopamine receptor (DRD2). The single largest risk factor that predisposes people to schizophrenia is genetic risk. The SNPs rs 1130214 and rs3730358 in AKT1 are functional and showed association with lower protein levels of AKT1 (Emamian et al., 2004). Thus AKT1 can participate in impaired dopaminergic neurotransmission and may be involved in the development of schizophrenia.

Objectives: The aim of the study was to determine the association of polymorphisms of AKT1 gene (rs1130214, rs3730358) with schizophrenia in the Russian population.

Methods: Blood samples were taken from 474 Russian Caucasian patients with a clinical diagnosis of schizophrenia (ICD-10: F20) and from 134 healthy persons. Genotyping of polymorphic variants of AKT1 gene (rs1130214, rs3730358) was carried out by real-time PCR. Results: Comparison of genotypes frequencies of AKT 1 gene rs1130214 and rs3730358 polymorphisms in schizophrenic patients and control group has not revealed statistically significant difference. The genotypes distribution has been shown as follows: GG $(44.7 \%$ and $49.3 \%)$, GT $(44.9 \%$ and $39.6 \%)$, TT $(10.3 \%$ and $11.2 \%)$ (rs1130214) and AA (2.7\% and 2.2\%), AG (26.8\% and 26.9\%), GG (70.5\% and 70.9\%) (rs3730358) in schizophrenic patients and healthy persons, respectively.

Conclusions: There is no association between AKT1 gene (rs1130214, rs3730358) polymorphisms and schizophrenia in Russian population. The reported study was funded by RFBR according to the research project № 18-315-20019. 
Conflict of interest: No

Keywords: AKT1; gene; polymorphism; schizophrenia

\section{Intellectual disability}

\section{EPP0591}

Serotoninergic toxicity triggered by low doses of sertraline in a patient with intellectual disability.

A. Giménez Palomo*, M. Garriga, M. Gómez-Ramiro, V. Soler, M. Vázquez and S.P. Gomes

Hospital Clínic, Psychiatry Barcelona, Spain

${ }^{\star}$ Corresponding author.

Introduction: Case report presentation of a 30 year-old patient with moderate intellectual disability and psychosis who is admitted to the Emergency Room after the development of a fluctuating clinical picture within the last 7 days characterized by mutism, perplexity and diaphoresis. His relatives confirm a regular pharmacological treatment with risperidone $1 \mathrm{mg}$ for the last 10 years and also report the addition of sertraline $25 \mathrm{mg} 8$ days ago. They do not explain other changes in his treatment or environmental stressors.

Objectives: The aim in this clinical case was oriented to discard a psychotic episode and diagnose and treat the current clinical picture. Methods: At the physical examination, the patient is ill-appearing, showing diaphoresis, tachycardia, low-grade fever and limited episodes to a few seconds in length of mutism and a blank stare, with no response to orders. There are no other pathological findings at the neurological exam. Aggressiveness, suspicion or other psychotic symptoms are not noticed, but disorganization in his behavior and fluctuating mental confusion are shown. Subsequently an acute urinary retention is found. A cranial scan and an electroencephalogram are carried out, not showing abnormalities. The blood test shows mild neutrophilia, with normal kidney function, creatine kinase and C-reactive protein levels. A lumbar puncture is carried out, not showing pathological findings.

Results: This clinical picture is oriented as a behavioral disturbance in context of serotoninergic toxicity according to the recent introduction of sertraline $25 \mathrm{mg}$.

Conclusions: After sertraline removal, his clinical situation improves reaching a normal behavior, so ciproheptadine treatment is no needed.

Conflict of interest: No

Keywords: serotoninergic toxicity; sertraline; E-POSTER PRESENTATION: INTELLECTUAL DISABILITY

\section{Mental health care - Part II}

\section{EPP0623}

Satisfaction with services provided for people affected by severe mental illness and their family members in azerbaijan.

\section{J. Ismayilova}

National Mental Health Center of the Ministry of Health, Consulting and Treatment, Baku, Azerbaijan
Introduction: Despite ongoing mental health reform taking place in Azerbaijan, the services for people suffering from Severe Mental Illness (SMI) are still underdeveloped. Available services are limited with traditional psychiatric interventions while psychological counseling and psychotherapy are not accessible for people with SMI. Objectives: The study aimed to measure patient's satisfaction with mental health services and their families as well as to find possible relations between satisfaction, sociodemografic and clinical variables.

Methods: Sociodemographic questionnaire, BPRS and Verona Service Satisfaction Scale-EU was completed by 50 patients and 50 family members.

Results: The overall satisfaction with services scores among patients and their family members was relatively high $(\mathrm{M}=3.9$; 95\% $\mathrm{CI}=3.65-4.15)$ and $(\mathrm{M}=4.02$; 95\% $\mathrm{CI}=3.83-4.21)$, and did not differ significantly (Wilcoxon $\mathrm{Z}=-0.738 ; \mathrm{p}=0.460$ ). Age of onset, duration of illness, number of hospitalizations and BPRS scores did not associated with overall satisfaction with services, but there was a correlation between overall satisfaction scores estimated by patients and number of episodes $\left(\mathrm{r}_{\mathrm{s}}=-0.301 ; \mathrm{p}<0.05\right)$. The more episodes a patient had, the less satisfaction with services he/she reported. Moreover, the majority of patients and their families are not informed about various services other than drug treatment.

Conclusions: The services related to finding employment and sheltered work are wished the most of all by the patients and their relatives. However these services are not provided yet. Likewise, desirable services include practical help at home, leisure activities and group sessions. As these services are not familiar to mental health care providers in our country, it will be beneficial to obtain international experience on establishing them.

Conflict of interest: No

Keywords: schizophrenia; services; family; satisfaction

\section{Mental health care}

\section{EPP0635}

Patients with cardiometabolic disorders associated with antipsychotics who receive pharmacological treatment for those adverse effects. Differences according to the kind of mental health unit

O. Olivas $^{1 \star}$, A. Arnaiz ${ }^{2}$, L. Erkoreka ${ }^{3}$, F. Lázaro ${ }^{4}$, T. Moreno ${ }^{5}$, N. Varela ${ }^{6}$, N. Basterreche ${ }^{2}$, M. Zumarraga ${ }^{7}$ and A. Arrue ${ }^{7}$

${ }^{1}$ Osakidetza, Red De Salud Mental De Bizkaia, Gernika. Bizkaia, Spain; ${ }^{2}$ Bizkaia Mental Health Network, Zamudio Hospital, Zamudio, Spain; ${ }^{3}$ Galdakao-Usansolo Hospital, Psychiatry, Galdakao, Spain; ${ }^{4}$ Red de Salud Mental de Bizkaia, Bermeo Hospital, Bermeo. Bizkaia, Spain;

${ }^{5}$ Galdakao_Usansolo Hospital, Osakidetza, Psiquiatria, Galdakao, Spain; ${ }^{6}$ Red de Salud Mental de Bizkaia, Uipo Tratamiento Asertivo Comunitario Uribe, Getxo. Bizkaia, Spain and ${ }^{7}$ Bizkaia Mental Health Network, Neurochemical Research Unit, Barakaldo, Spain ${ }^{\star}$ Corresponding author.

Introduction: Treatment with antipsychotics can induce the development of obesity and metabolic syndrome (MS) that are often not treated properly; all of this contributes to the development of serious chronic health conditions

Objectives: To assess parameters that define metabolic syndrome (MS) in patients treated with antipsychotics and to estimate the 
percentage of patients receiving treatment for these cardiometabolic disturbances, according to the kind of psychiatric center where they receive care.

Methods: We included 214 patients, 62 women and 152 men, treated with atypical antipsychotics for a minimum period of three months. The proportion of patients with altered parameters and in treatment according to the center was compared.

Results: Table 1 shows the percentage of patients who present altered values in waist, in the rest of MS parameters, MS (3/5 criteria) and who receive pharmacological treatment according to the unit. Of the 161 patients with metabolic and/or blood pressure alterations, only 47 received treatment. No differences were observed in the percentage of patients treated according to the care center $(\mathrm{x} 2=3.94, \mathrm{p}=0.2685)$

Conclusions: In our health system, with free and universal access to healthcare, there are still a high percentage of patients with untreated side effects in units where they receive daily care as well as in outpatient units. It is necessary to improve the physical health control of psychiatric patients and the integration of patients in the primary health care services.

\section{Conflict of interest: No}

Keywords: antipsychotic; cardiometabolic disturbances; metabolic syndrome

\section{Mental health care - Part III}

\section{EPP0637}

Joint planning of discharge people with severe mental illnesses as a future model of continuity care and more quality mental health services

\section{G. Racetovic ${ }^{1 \star}$, M. Latinovic ${ }^{2}$, S. Branka ${ }^{3}$ and E. Mesic ${ }^{4}$ \\ ${ }^{1}$ Health Center Prijedor, Community Mental Health Center, Prijedor, Bosnia and Herzegovina; ${ }^{2}$ Ministry of Health and Social Welfare of Republic of Srpska, Department for Hospital Care, Banja Luka, Bosnia and Herzegovina; ${ }^{3}$ Ministry of Health and Social Welfare of Republic of Srpska, Department for Social, Family and Child Welfare, Banja Luka, Bosnia and Herzegovina and ${ }^{4}$ Asocijacija XY, Project Of Mental Health In Bosnia and Herzegovina, Sarajevo, Bosnia and Herzegovina \\ ${ }^{\star}$ Corresponding author.}

Introduction: In Bosnia and Herzegovina (BH) was noted that transparent and closer collaboration between hospital and community sectors is not developed enough beside very good network of 78 community mental health centers (CMHC) and almost 20 hospital/clinical departments of diferent level. Leaders of continuous care should be CMHCs, but still are present diferences between sectors, mostly in defining of they roles even they need to be more collaborative and transparent and patient oriented. Objectives: Third phase of the Mental Health Project in $\mathrm{BH}$ (MHPBH) focused also to bridging the gaps between hospitals and $\mathrm{CMHC}$ as providers of continuity of care trough creation of guidance for joint planning of discharge from hospital of people with broad spectrum of needs (medical, social and economic).

Methods: Review of proposed document of joint planing of discharge from the hospital developed in one $\mathrm{BH}$ entity by Task Force apponted by the entity Minister of Health.
Results: First draft includes reccomendations for joint planing discharge using good results of the reform in $\mathrm{BH}$ (i.e., case management). Pilot implementation of 6 months in three diferent hospitals and 10 CMHC were conducted in 2019 and final evaluation gave new practical inputs for finalization of the document and its connection with new $\mathrm{BH}$ mental health legislative.

Conclusions: Collaboration between services in the community must be continuous, transparent and always leading with users needs and their actual situation considering all kinds of the support after discharge fom the hospital. Planing in advance is the best way to realize those tasks for our patients.

Conflict of interest: No

Keywords: Discharge; Community; Collaboration; Hospital

\section{Mental health policies}

\section{EPP0657}

Integrating mental health in primary health care, success story (mental health gap action program (mhgap), egypt /2019)

\section{S. Noby}

General Secretariat of Mental Health \& Addiction Treatment, Ministry of Health, Egypt, Director Of Mental Health Gap Action Program (mhgap) Egypt, Executive Director Of Research Unit At Gsmhat., Cairo, Egypt

Introduction: Mental health services are the means by which effective interventions for mental health are delivered. The way these services are organized has an important bearing on their effectiveness and ultimately on whether they meet the aims and objectives of a mental health policy. The recently adopted national insurance system in Egypt could be a great opportunity for mhGAP implementation. Primary care sector would be more relied upon for early detection and management of mental health disorders as an integrated part of the new health system. Also trained medical personnel would be more sustainable in PHCs as the rapid turnover of medical personnel was found to be the greatest challenge facing the previous mhGAP implementation.

Objectives: To implement a more integrative and sustainable mhGAP in the primary health care centers in the light of the newly adopted health insurance system in the country.

Methods: Designing a plan of action for MHGAP implementation. - MHGAP TOT training. · Training of primary health care medical personnel on adapted MHGAP modules- Implementation of MHGAP in three governorates in Egypt. · Support, supervision and evaluation.

Results: Ministry of health and WHO country office in Egypt released a National recommendations for integration of mental health services in primary care sector in Egypt/2019. Description of integration of Mental health services in primary care sector in three governorates of Egypt (Port Said, Ismailia and Damietta) in 2019.

Conclusions: For the first time in Egypt, Primary care unit start to be the first gate to provide mental health services for psychiatric patients.

\section{Conflict of interest: No}




\section{Neuroscience in psychiatry - Part I}

\section{EPP0684}

\section{The clinical case of a 53-year-old-woman with atypical cognitive symptoms.}

F. De Oliveira Azevedo ${ }^{1 \star}$, M. Fernandes ${ }^{2}, H$. Barreto ${ }^{1}$ and A.S. Sequeira

${ }^{1}$ Centro Hospitalar Lisboa Ocidental, Psiquiatria E Saúde Mental, Lisboa, Portugal and ${ }^{2}$ Centro Hospitalar Lisboa Ocidental, Neurologia, Lisboa, Portugal

${ }^{*}$ Corresponding author.

Introduction: Early-onset Alzheimer's Disease(AD) may present atypical cognitive symptoms. Affective symptoms are common, so they can be misdiagnosed as depression-related Pseudodementia.

Objectives: To report the case of a patient with atypical cognitive symptoms diagnosed with AD.

Methods: We describe the patient's clinical history, diagnostic investigation and therapeutic approach. We searched the "PubMed" database with the terms "early onset" "AD".

Results: A 53-year-old woman was admitted to a psychiatry outpatient clinic for cognitive symptoms. For 2 years her family noticed periods of disorientation, memory problems, difficulty cooking and managing medication. She abandoned her teaching work as she felt incapable of performing her job. Nevertheless, she was able to leave the house alone and go grocery shopping. No family history of neurodegenerative diseases. One year ago she was diagnosed pseudodementia and medicated with sertraline200mg, bupoprion300mg and zotepine40mg with improvements in energy and apathy. Although, the cognitive complaints worsened over time. Mental state examination showed hyperfamiliarity, difficulty retaining/evoking information and anomic pauses. She was still able to carry her daily basic functions but the MMSE score was 11/30 and MoCA 3/10. A neuropsychological evaluation showed inconsistent findings. Brain-MRI presented bilateral cortical atrophy (anterior temporal and parietal lobes). She was referred to neurology and a CSF-examination showed decreased $\beta$-amiloid $(411 \mathrm{pg} / \mathrm{mol})$, increased t-tau $(493 \mathrm{pg} / \mathrm{mol})$ and $\mathrm{p}$-tau $(81.5 \mathrm{pg} / \mathrm{mol})$, compatible with $\mathrm{AD}$.

Conclusions: Cognitive complaints, especially in younger patients with no family history of neurodegenerative diseases, can be challenging and need a multidisciplinary approach. The correct diagnosis is essential and implies different psychopharmacological approaches, family education and care planning.

Conflict of interest: No

Keywords: neurology; Alzheimer; Pseudodementia; dementia

\section{EPP0685}

Dementia or pseudodementia in a 45 -year-old woman with a zolpidem dependence

F. De Oliveira Azevedo ${ }^{1 *}$, M. Fernandes ${ }^{2}$, C. Spínola ${ }^{1}$ and C. Almeida ${ }^{1}$

${ }^{1}$ Centro Hospitalar de Lisboa Ocidental, Departamento De Psiquiatria E Saúde Mental, Lisboa, Portugal and ${ }^{2}$ Centro Hospitalar Lisboa Ocidental, Neurologia, Lisboa, Portugal

${ }^{*}$ Corresponding author.

Introduction: The differential diagnosis between depression and dementia is usually prompted in the elderly.
Objectives: We present the case of a 45-year-old woman with zolpidem dependence, insomnia, apathy and applanated affect with brain CT abnormalities.

Methods: Records examination, and review of literature

Results: A 45 -year-old woman was admitted with a $200 \mathrm{mg}$ zolpidem intoxication. As motive she stated, "I was only trying to sleep". She had a depression following, taking $300 \mathrm{mg}$ venlafaxine, $300 \mathrm{mg}$ trazodone, $100 \mathrm{mg}$ quetiapine and $10 \mathrm{mg}$ zolpidem. Lately she abandoned all medication except zolpidem, increasing dosage to $50-60 \mathrm{mg} / \mathrm{d}$, sleeping a few interrupted hours. She presented psychomotor-retardation, with vague speech, applanated affect, indifference, mild cognitive complaints, and insomnia. Neurological examination showed primitive signs. She had three provoked tonic-clonic seizures related to zolpidem withdrawal. CT-scan revealed bilateral frontal atrophy. RM-CE showed findings suggestive of sagging brain syndrome, possibly mimicking bilateral frontal atrophy. CSF-examination revealed normal opening pressure $(15 \mathrm{~cm}-\mathrm{H} 2 \mathrm{O})$ with no abnormal findings. EEG showed global slowing with 7-8 $\mathrm{Hz}$ basal activity, without epileptiform discharges. She responded partially to antidepressants and is currently awaiting further evaluation in neurology outpatient clinic. Conclusions: Behavioral variant frontotemporal dementia (FTD) is characterized by a young onset and progressive changes in personality, cognition, impulse control and social behavior. Additional symptoms can include depressed mood, apathy, and anhedonia. When evaluating patients with depression, additional workup to exclude neurodegenerative diseases such as FTD should be considered. Certain types of dementia, especially with early presentations can mask or coexist with affective disorders, with clinical suspicion and multidisciplinary work being paramount for correct diagnosis and treatment.

\section{Conflict of interest: No}

Keywords: neurology; frontotemporal dementia; Pseudodementia; Zolpidem

\section{EPP0688}

'Research domain criteria' in context: $\mathrm{pi} 3 \mathrm{k} / \mathrm{pdk1} / \mathrm{pkb}$ signaling pathway, fear, anxiety and cognitive deficits

L. Giménez-Llort ${ }^{1 *}$, M. Santana-Santana ${ }^{2}$ and J.R. Bayascas ${ }^{3}$

${ }^{1}$ Universitat Autònoma de Barcelona, Institute Of Neuroscience \& Department of Psychiatry and Forensic Medicine, Barcelona, Spain;

${ }^{2}$ Universitat Autònoma de Barcelona, Institute Of Neuroscience \& Dept Of Psychiatry and Forensic Medicine, Barcelona, Spain and ${ }^{3}$ Universitat Autònoma de Barcelona, Institut Of Neurosciences \& Department of Biochemistry and Molecular Biology, Barcelona, Spain ${ }^{*}$ Corresponding author.

Introduction: The 'Research Domain Criteria' proposes a new biological psychiatry conceptualization of mental health and disease, considering phenotypic differences among disorders as explained by variations in the nature and degree of neural circuitry disruptions, under the modulation of several developmental, compensatory, environmental and epigenetic factors. Thus, dysfunction and dysregulation at genetic, neural and behavioral levels point at fine-tuning of broadly spread networks as critical for a wide array of behaviors and mental processes.

Objectives: In this context, we aim to characterize the behavioral domains of the PI3K/PDK1/PKB signaling pathway known to play an essential role in the regulation of cellular processes, leading to diverse physiological responses. 
Methods: Behavioral and functional screening of young and mature male and female PDK1 K65E knock-in mice.

Results: The double mutation of the PDK1 PH-domain results in an enhancement of negative valence system. That is, responses of fear, and anxiety-like behaviors which seems to be specific of young adulthood, found regulated at middle-age. In contrast, cognitive deficits as measured in a spatial working memory task, were found at both ages, and independently of their anxious-like profiles. This would be in agreement with the distinct cortical and hippocampal deficits in the Akt signaling found in the brain of these animals. The modulation by sex factor deserve further exploration.

Conclusions: The present results contribute to the in vivo characterization of the behavioral impact of $\mathrm{PKB} / \mathrm{Akt}$ signal pathway dysfunction and support the conceptualization of qualitative and quantitative variations of neural circuit disruptions underpinning phenotypic differences that are relevant to several mental disorders.

Conflict of interest: No

Keywords: Animal models; Negative Valence Systems; Behavioral neuroscience; RDoC

\section{Obsessive-compulsive disorder}

\section{EPP0706}

\section{Cognitive-behavioural conceptualization of e-poster presentation: obsessive-compulsive disorder}

\section{Fernandes Santos*, C. Miranda and A.B. Medeiros}

Hospital Garcia de Orta, Department of Psychiatry and Mental Health, Almada, Portugal

${ }^{\star}$ Corresponding author.

Introduction: Cognitive-behavioural therapy is effective in the treatment of obsessive-compulsive disorder (OCD), being recommended as first-line intervention in international guidelines. The understanding of OCD according to the cognitive-behavioural theory relies on works of different authors, namely Rachman and Salkovskis, who, since the 1970s, have developed an explanatory model of the disease.

Objectives: To review the conceptual model of OCD according to cognitive-behavioural theory, which explains a wide range of clinical phenomena and constitutes the basis for psychotherapeutic approach. Methods: Non-systematic review of literature through search on PubMed/MEDLINE electronic database, as well as on reference textbooks.

Results: Obsessions are conceptualized as normal intrusive thoughts, misinterpreted as a sign of harm to oneself or others, about which the patient feels responsible (or its prevention). This interpretation causes adverse mood, focusing attention on intrusions and environmental stimuli that may increase their occurrence. The inflated sense of responsibility leads the person to develop neutralizing behaviours, characterized by preoccupation and over-control, in addition to counterproductive safety strategies. These responses contribute not only to the maintenance of negative beliefs, but also to the emergence of further intrusion and perceived threat and responsibility, in a cycle of negative thinking and neutralization that reinforces OCD. There are key cognitions in patients with OCD: thought-action fusion, hyperactivated personal responsibility schemes, control of thoughts, perfectionism, overestimation of threat and intolerance to uncertainty.
Conclusions: The cognitive-behavioural model of OCD has produced a large body of empirical support and has led to the development of valuable treatments. It is important to understand its theoretical bases.

\section{Conflict of interest: No}

Keywords: obsessive-compulsive disorder; cognitive-behavioural model; cognitive-behavioural therapy

\section{Old age psychiatry - Part I}

\section{EPP0713}

\section{Systematic review of clinical evidence for the use of antipsychotic medication in the treatment of delirium}

J. Facucho-Oliveira ${ }^{\star}$, P. Espada-Santos, D. Esteves-Sousa, M. Albuquerque, M. Costa, A. Moutinho, P. Cintra, L. Mendonça and J. Tropa

Hospital de Cascais, Department of Psychiatry, Cascais, Portugal ${ }^{*}$ Corresponding author.

Introduction: Delirium is a neuropsychiatric syndrome with a prevalence of approximately $20 \%$ of hospitalized patients and is most frequently related to an underlying medical insult. Delirium is characterized by acute onset and characterized by fluctuant disabilities in attention, awareness and orientation along with cognitive disturbances in memory and perception. Despite no pharmacologic therapy are specifically approved, clinicians have used antiphsycotics (AP) to manage the agitation and the psychotic features of delirium for many decades.

Objectives: A systematic literature review attempts to elucidate whether treatment of hospitalized patients with delirium using AP improve clinical outcomes.

Methods: A literature search was conducted in PubMed to identify published randomized controlled trials (RCTs), prospective and case-control studies. Inclusion criteria included a literature focusing on treatment of delirium of adult inpatient. Data was reviewed, analyzed and discussed.

Results: Most recent data arising from large RCT and meta-analysis do not determine considerable clinical benefits comparing treatment of delirium with antipsychotics to placebo. Delirium duration, sedation, hospital length of stay and mortality was not found to be significantly different. Data on cognitive functioning, delirium severity and neurologic injuries also points to a limited benefit of the use of AP. Conclusions: There is insufficient evidence to support the routine use of AP to prevent or treat delirium in hospitalized patients. Clinical trials with specific protocols for prevention and management of delirium should perhaps focus on assessing risk of developing delirium along with the implemention of less dynamic environment with steady sensorial stimulation and increased nursing.

Conflict of interest: No

Keywords: Delirium; antipsychotic medication

\section{EPP0716}

How are we using twitter when referring to alzheimer?

M. Alvarez-Mon ${ }^{1 \star}$, M. Llavero-Valero ${ }^{2}$, V. Pereira-Sanchez ${ }^{3}$, R. Sanchez-Bayona ${ }^{1}$, M. Vallejo-Valdivielso ${ }^{4}$, J. Monserrat ${ }^{5}$, G. Lahera ${ }^{6}$, A. Asunsolo Del Barco ${ }^{5}$ and M. Alvarez-Mon ${ }^{5}$ 
${ }^{1}$ University of Navarra Clinic, Department of Psychiatry, Pamplona, Spain; ${ }^{2}$ University of Navarra Clinic, Department of Endocrinology, Madrid, Spain; ${ }^{3}$ New York University, Langone Child Study Center, New York, United States of America; ${ }^{4}$ University of Navarra Clinic, Child \& Adolescent Psychiatry Unit, Department of Psychiatry and Clinical Psychology, Pamplona, Spain; ${ }^{5}$ University of Alcala,

Department of Medicine and Medical Specialties, Madrid, Spain and ${ }^{6}$ University of Alcala, School Of Medicine, Alcalá de Henares, Spain ${ }^{*}$ Corresponding author.

Introduction: The analysis of tweets is a recent relevant area of study for understanding the sentiments of society, patients and health care providers.

Objectives: Analyze the content of tweets referring to \#Alzheimer. Methods: We focused on searching for tweets that referred to Alzheimer over a period of eight consecutive days in 2018. Each tweet's content was rated as nonmedical (NM: testimonies, health care products, advocacy or misuse) or medical (M: included a reference to the illness's diagnosis, treatment, prognosis, or prevention). NM tweets were classified as positive or pejorative; we also evaluated the appropriateness of the medical content.

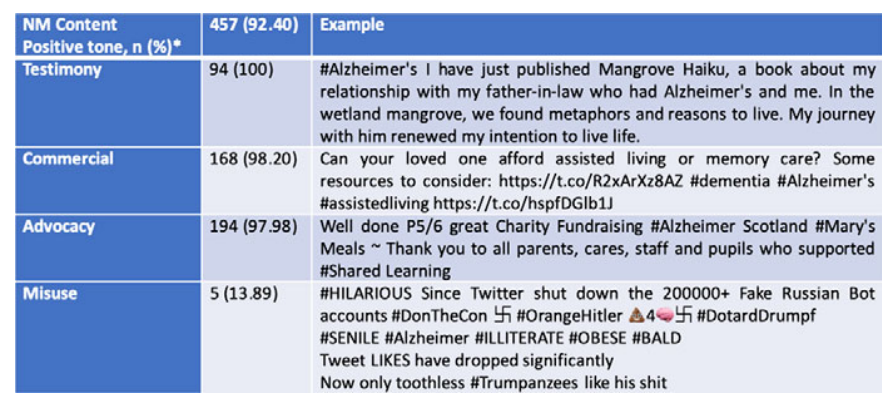

Results: We analyzed a total of 930 tweets: 374 were classified as M and 457 as NM. The medical content focused on the prevention of Alzheimer (76.74\%) and showed high scientific appropriateness $(89.05 \%)$. Non-Medical content (Table 1) focused on advocacy (42.45\%). Pejorative content was minimal (7.60\%). The hashtags most frequently associated with \#Alzheimer were: dementia, health, brain, care, memory, caregiver, aging and science.

Conclusions: Twitter is used as a relevant communication tool for distributing medically accurate information for the disorder as well as for advocacy efforts.

Conflict of interest: No

Keywords: Twitter; Advocacy; Prevention; Alzheimer

\section{Old age psychiatry - Part IV}

\section{EPP0746}

Description of the long-term individual course of neuropsychiatric symptoms in people with alzheimer's and lewy body dementia

\footnotetext{
A. Vik-Mo ${ }^{1 \star}$, M. Borda ${ }^{2}$ and D. Aarsland ${ }^{3}$

${ }^{1}$ Centre of Age-related Medicine, Centre Of Age-related Medicine, Randaberg, Norway; ${ }^{2}$ King's College London, Department of Old Age Psychiatry, Institute Of Psychiatry, Psychology and Neuroscience,
}

United Kingdom and ${ }^{3}$ Centre of Age-related Medicine, Stavanger University Hospital, Norway, stavanger, Norway ${ }^{*}$ Corresponding author.

Introduction: Understanding the natural course of NPS in dementia is important for planning patient care and trial design, but few studies have described the long-term course of Neuropsychiatric symptoms (NPS) in individual patients.

Objectives: We describe the course of NPS in patients with Alzheimer's disease $(\mathrm{AD})$ and Lewy-body dementia (LBD) from time of diagnosis until death.

Methods: Primary inclusion of 223 suspected mild dementia patients from general practice were followed with annual assessment including the Neuropsychiatric Inventory (NPI) for up to 12 years. Total and item NPI were classified as stable, relapsing, single episodic or not present based on 4.96 (SD 2.3) observations (98\% completeness of longitudinal data) for the $113 \mathrm{AD}$ and 84 LBD included.

Results: Eighty \% had stable NPI total $>1$, while $50 \%$ had stable modest NPI total $>12$ and $25 \%$ stable NPI total $>24$ NPI total scores. Very severe NPS ( $>48)$ were mostly single episodes, but $8 \%$ of $\mathrm{AD}$ patients had stable severe NPS. The AD patients with the highest 20\% NPI total scores had more stable or relapsing course of four key symptoms: aberrant motor behaviour, aggression/agitation, delusions, and irritability (Odds ratio 55, $\mathrm{p}<0.001$ ). This was not seen in DLB. Fifty-seven $\%$ of AD and $84 \%$ of DLB patients had reoccurring psychotic symptoms.

Conclusions: We observed a highly individual course of NPS, with most NPS presenting as a single episode or re-occurring, while a stable course was less common, especially in LBD. These findings demonstrate the importance of an individualized approach, i.e. personalized medicine, in dementia care.

Conflict of interest: No

Keywords: dementia; affective; psychosis; Neuropsychiatric

\section{EPP0750}

Brain aging and frontal lobe disorders - a review of the current evidence

\section{R. Valido* and P. Ferreira}

Hospital de Magalhães Lemos, Psychiatry, Porto, Portugal ${ }^{\star}$ Corresponding author.

Introduction: The aging brain as always been mysterious. However, in the last decades with the advances in neuroimaging and neuropsychology comprehension, there are new important insights about what brain aging is and what it implies.

Objectives: We aim to review the most up to date scientific knowledge about brain aging and demonstrate how clinically relevant this knowledge is to the XXI century Psychiatrist.

Methods: We performed a literature review using Pubmed databases and UpToDate.

Results: The frontotemporal areas are especially prone to the effects of aging, with some degree of atrophy being expected in normal aging. White matter integrity is also compromised in the aging brain. Age specifically exacerbates executive impairment after frontal injuries. The results also demonstrated that age significantly predicted the degree of global brain atrophy and white matter lesions. In normal aging, it is expected that there will be a progression towards the deterioration of processing speed, executive functions and episodic memory with relative 
preservation of verbal skills and knowledge of the surrounding world. Different regions of the Prefrontal Cortex when dysfunctional can present clinically as different syndromes, particularly apathy, disinhibited or the dysexecutive types.

Conclusions: Frontal Lobe functioning assessment is very important and should be implemented in all patients beyond 40 years of age with a history of significant risk factors for accelerated brain aging (for example head trauma, stroke or long life depression). The knowledge that specific domains of executive function are affected not only have diagnostic value as it can further inform treatment strategies.

Conflict of interest: No

Keywords: brain aging; executive impairment; frontotemporal regions; Prefrontal Cortex

\section{Oncology and psychiatry}

\section{EPP0753}

\section{Depression and cancer - a bidirectional relationship}

R. Barranha*, J. Quarenta and H. Pinto

Centro Hospitalar do Tâmega e Sousa, Department of Psychiatry and Mental Health, Penafiel, Portugal

${ }^{\star}$ Corresponding author.

Introduction: Cancer has a high rate of comorbidity with depressive disorders, with an estimated $16.3 \%$ of cancer patients suffering from major depression. Given the perceived existential threat associated with the disease and its treatment, the increased risk of depressive disorders is understandable. However, some researchers have recently proposed that the connection between these two diseases might not be unidirectional.

Objectives: To explore the biological mechanisms underlying the relationship between cancer and depression. To assess the impact of depression on cancer incidence, progression and mortality.

Methods: We undertook a literature review by searching in PubMed articles written in English. The key terms used were "cancer", "major depressive disorder", "survival" and "inflammation".

Results: There is mounting evidence that inflammation, particularly through proinflammatory cytokines, is involved in depression. That has been proved in cancer patients, in which the elevation of cytokines correlates with depressive symptoms. On the other hand, low-grade chronic inflammation also plays a role in the pathophysiology of cancer. This interplay has been corroborated by one large population-based epidemiological study that found an association of a lifetime history of major depression with a higher risk for cancer, and a statistically significant increase in risk for breast cancer. It has also been revealed that sustained depression after a cancer diagnosis influences cancer progression and shortens survival. Similarly, it is believed that this effect is mediated by the release of proinflammatory cytokines.

Conclusions: Proinflammatory cytokines may mediate a two-way relationship between cancer and depression. Further research is needed to assess the impact of depression treatment on cancer survival.

Conflict of interest: No

Keywords: major depressive disorder; cancer; Survival; Inflammation

\section{Others - Part I}

\section{EPP0770}

Mach-iv (test of machiavellianism): psychometric properties and normative data from the portuguese population

\author{
M. Esteves-Pereira ${ }^{1 \star}$, A. Azeredo ${ }^{2}$, D. Moreira $^{3}$, F. Almeida ${ }^{4}$ and \\ I. Brandão ${ }^{5}$ \\ ${ }^{1}$ University of Porto, Faculty Of Medicine, Porto, Portugal; ${ }^{2}$ University \\ of Porto, Laboratory Of Neuropsychophysiology- Faculty Of \\ Psychology and Educational Sciences, Porto, Portugal; ${ }^{3}$ University of \\ Porto, Laboratory Of Neuropsychophysiology- Faculty Of Psychology \\ and Educational Sciences, Porto, Porto, Portugal and ${ }^{4}$ Maia University \\ Institute, Social and Behavioral Sciences Department, Maia, Portugal, \\ ${ }^{5}$ University of Porto, Faculdade De Medicina, Porto, Portugal \\ ${ }^{*}$ Corresponding author.
}

Introduction: Machiavellianism addresses a distrustful view of the world, a disposition of the individual behaving in an unethical way, and a repertoire of manipulative tactics that can be used to secure power and gains for oneself.

Objectives: The main objective in this study was to assess the structure of the MACH-IV Scale, fitting various measurement models, especially those that integrate the distinction tactics-views, with the aspect of positively and negatively worded items.

Methods: We performed basic item and scales psychometric characteristics, as well as confirmatory and exploratory factor analyses in a Portuguese sample $(\mathrm{N}=293$; Mage $=35.3$; $\mathrm{SD}=12.6)$.

Results: The original factor structure of the MACH-IV was not confirmed in our sample. After exploratory factor analysis, a strong model fit was obtained. All factors (Positive Interpersonal Tactics, Negative Interpersonal Tactics, Positive View of Human Nature, and Cynical View of Human Nature) presented adequate internal consistency. As the psychometric analyses provided good evidence for the quality of this version of the scale, normative data is provided by sex, age, and school grade.

Conclusions: Based on the results: (a) the Portuguese version of MACH-IV may be easily applicable for clinicians, and (b) we presented average scores by sex, age, and education to provide normative data to facilitate its clinical use.

\section{Conflict of interest: No}

Keywords: MACH-IV; Test of Machiavellianism; Portuguese population; Personality Tests

\section{EPP0773}

Psychiatric conditions in sports: when stigma wins the match

\section{F. Caetano}

Hospital de Magalhães Lemos, Serviço De Internamento B, Porto, Portugal

Introduction: Mental health stigma is still a pivotal health problem in today's society. In sports, stigma can prevent athletes from seeking treatment and reaching their full potential. Moreover, this specific population has been rarely analyzed in the literature. 
Objectives: Review the existing literature on psychiatric conditions in sports, its trends and the associated stigma.

Methods: Literature search on PubMed and Google Scholar.

Results: While athletes might be less likely to develop certain psychiatric disorders, eating disorders, adjustment disorders and substance abuse are sometimes more common in this population. In addition to these more traditional diagnoses, the overtraining syndrome is a prototypical sport psychiatric illness with physical components, social impacts, and mental stress. Sports psychiatry is rapidly evolving. Using the keywords "sport psychiatry" and "stigma", we find an increasing trend in the number of articles available in Google Scholar over the years. For instance, from 1991 to 2000, only one article was published; from 2001 to 2010, 42 articles were published; from 2011 onwards, 167 articles were published. However, like other fields of psychiatry, stigma regarding mental health is the most common barrier to seek treatment. Factors such as perceived stigma, confidence in consolation, cultural preference, and openness have been shown to predict the likelihood of coaches to refer their players to a mental health professional

Conclusions: Recently professional athletes have been more vocal about their mental health struggles, such as the Italian goalkeeper Buffon. These testimonies, as well as quality research and well trained mental health professionals can help mitigate stigma.

Conflict of interest: No

Keywords: Stigma; Sports psychiatry

\section{Others - Part III}

\section{EPP0794}

Heterogeneity in the diagnostics of attention deficit hyperactivity disorder diagnosis in the Czech Republic among adults

\section{Vňuková, R. Ptáček and J. Raboch}

First Faculty of Medicine, Psychiatry, Prague, Czech Republic ${ }^{\star}$ Corresponding author.

Introduction: Attention Deficit Hyperactivity Disorder (ADHD) is a neurological disorder most commonly diagnosed at the age of 7-10 years, which persists in adulthood in $40-60 \%$.

Objectives: Previous years we reported the discrepancy of the diagnosis among children aged 6-10, as a follow up we looked at the prevalence and source of diagnosis among adults.

Methods: Data were collected using a combination of qualitative and quantitative methodology. In the first wave we used standardized questionnaires for symptomatology of ADHD, time perception as well as lifestyle and demographic surveys.

Results: A total of 1518 respondents participated in the research: 766 men and 752 women. $3 \%$ of respondents ( 31 men and 15 women) reported having been diagnosed with ADHD / hyperkinetic disorder throughout their lives. The average age at which they were diagnosed was 9.18 years. Most often, up to $39.1 \%$, the diagnosis came from an expert in the pedagogical-psychological counseling, followed by a child psychiatrist (21.7\%) and a general practitioner (19.6\%). Only in one case was the diagnosis made by a clinical psychologist.

Conclusions: The worldwide prevalence of ADHD is between 3-7\%. Our results suggest an adequate level of $\mathrm{ADHD}$ diagnosis. Similarly, as with children and adolescents, the problem may be that the diagnosis came from a number of sources. However, with children we saw that there was no ADHD diagnosed by general practitioner and far more cases were diagnosed by clinical psychologist. Therefore, we see further discrepancy even within Czech republic. This is only further proof that implementation of structured assessment is crucial.

Disclosure: Financial support provided by grant : GA ČR 18 11247S (research grant titled: ADHD symptomatology and Time perception)

Keywords: ADHD; diagnosis; structured assessment

\section{EPP0802}

\section{Mental health and climate change in the literature}

S. Ramos ${ }^{\star}$, D. Sousa and J. Mendes

Unidade Local de Saúde da Guarda, E.P.E., Psychiatry, Guarda, Portugal

${ }^{\star}$ Corresponding author.

Introduction: In the week of the Global Climate Strike, health professionals are hasted to prepare for the consequences of climate change. The hazards of climate change in human health is well documented and the consequences on the mental health of the populations has received increased attention by the scientific community in recent years.

Objectives: To characterize the available literature on climate change and mental health.

Methods: We analyzed the database PubMed for literature regarding climate change and mental health using the search terms climate change, global warming, mental health, psychiatry, depression and anxiety. We characterized our sample with descriptive statistics.

Results: We obtained a sample of 163 articles which fit inclusion criteria. We analyzed the evolution of literature production by year (figure 1) and observed an increase in the number of publications in recent years. Regarding the type of publication, 60 (36.9\%) were research articles, 8 (4.9\%) systematic-reviews or meta-analysis, $61(37.4 \%)$ non-systematic reviews, 17 (10.4\%) opinion articles and 17 (10.4\%) consisted in other types of publications. Most publications originated from North America (37.4\%), followed by Australia (28.8\%), Europe (23.3\%), Asia (9.8\%) and Africa (0.6\%). We did not found publications from South America in our sample. Most publications were written in English (95.7\%).

Conclusions: The number of publications concerning mental health and climate change is increasing. We observed an asymmetry in the publications' origins and an abundance of non-systematic reviews. Further studies are necessary to understand the consequences of climate change around the world and its impact on mental health.

Conflict of interest: No

Keywords: Climate Change; mental health

Pain

\section{EPP0805}

Fibromyalgia and bipolar affective disorder: a significant influence?

J. Brás*, R. Sousa, A. Costa, B. Guedes, A. Costa and N. Gil 
Centro Hospitalar Tondela-Viseu, Department of Psychiatry and Mental Health, VISEU, Portugal

${ }^{\star}$ Corresponding author.

Introduction: Fibromyalgia (FM) is a debilitating syndrome that affects muscles and soft tissues. It is characterized by the presence of chronic widespread pain associated with fatigue, sleep problems and psychological symptoms, including depression and anxiety. A large proportion of patients with FM have co-morbid mental disorders. The literature reported a strong association between FM and major depressive disorder, however the connection with bipolar affective disorder (BAD) is not so well known. The comorbidity with $\mathrm{BAD}$ have crucial implications for FM treatment because antidepressants used in FM treatment may induce mood destabilization, manic switches and episodes with mixed features.

Objectives: To present a non-systematic review of the literature about the relationship between fibromyalgia and bipolar affective disorder, particularly epidemiological associations and shared pathophysiological aspects.

Methods: A literature search was conducted using the search engine Pubmed ${ }^{\circledR}$ and the keywords: fibromyalgia and bipolar affective disorder.

Results: FM is closely associated with BD, with clinical implications. Studies suggested a prevalence of BD in FM patients of approximately $21 \%$. Furthermore, a myriad of studies proved the existence of a pathophysiological overlap among these two pathologies. Several hypotheses explain this connection, for example shared abnormalities in cortical areas involved in pain processing and emotional control, a hyperactivation of the hypothalamicpituitary-adrenal axis and an impaired glucorticoid receptor mediated signaling. Moreover, FM and BAD are characterized by chronic low-grade inflammation and share genetic polimorphisms in the monoaminergic systems.

Conclusions: The literature proves that exists a clear relationship between FM and BAD. These association has several clinical implications both in diagnosis and treatment.

Conflict of interest: No

Keywords: Bipolar disorder; fibromyalgia

\section{Personality and personality disorders - Part II}

\section{EPP0819}

\section{Personality traits among regular and occasional pokémon go users}

A. Giménez Palomo ${ }^{1 \star}$, G. Anmella ${ }^{2}$, J. Pinzon Espinosa ${ }^{3}$, S. P. Gomes ${ }^{4}$ and I. Pacchiarotti ${ }^{5}$

${ }^{1}$ Hospital Clínic, Psychiatry, Barcelona, Spain; ${ }^{2}$ Hospital Clinic, University of Barcelona, IDIBAPS, CIBERSAM, Schizophrenia Unit, Institute Of Neuroscience, Barcelona, Spain; ${ }^{3}$ Hospital Clinic de Barcelona, Institut Clinic De Neurociencias, Barcelona, Spain; ${ }^{4}$ Hospital Clinic de Barcelona, Psychiatry, Barcelona, Spain and ${ }^{5}$ Bipolar and Depressive Disorders Unit, Hospital Clínic de Barcelona, Psychiatry, Barcelona, Spain

${ }^{\star}$ Corresponding author.

Introduction: Pokémon Go is an augmented reality game that encourages players to walk and attend determined spots in order to battle in raids with other players. So far, poor attention has been paid on personality characteristics of this population and the game's benefits in sociability.

Objectives: To describe sociodemographic characteristics of population playing Pokémon Go and assess the impact of personality traits on the game outcomes.

Methods: A survey was distributed to Pokémon Go groups via different social networks from Spain throughout August of 2019. Descriptive analyses were conducted with SPSS v. 23.0.

Results: 1,211 individuals completed the survey, aged on average 30.8 years old. $64.9 \%$ were men and $35.1 \%$ women. $44.3 \%$ had reached the highest level (40). 33.8\% of participants referred substance consumption daily. They were more likely to have received psychiatric treatment than non-consumers. Level 40 players significantly referred less psychiatric consultations than non-level $40.48 .8 \%$ of subjects reported cluster A, 28.2\% cluster B, and $50 \%$ cluster $C$ personality traits. Level 40 participants were more unlikely to have cluster B personality traits $(p=0.037)$ than non-40. Women were more unlikely to have cluster A $(\mathrm{p}<0.001)$ but more likely to have cluster $\mathrm{C}(\mathrm{p}=0.01)$ traits than men. Cluster A predisposed to more reported harassement $(p=0.01)$ but not conflicts than non-cluster A, whilst cluster B predisposed to more harassment $(\mathrm{p}<0.001)$ and conflicts $(\mathrm{p}<0.001)$, and cluster $\mathrm{C}$ to none of them.

Conclusions: A better knowledge of players' characteristics could be useful for the future development of applications as a tool to better motivate subjects for specific interventions.

\section{Conflict of interest: No}

Keywords: Pokémon Go; Personality traits; substance use; sociability

\section{EPP0822}

\section{Relationship between dispositional optimism and road rage in adult}

A. Syed ${ }^{1}$, A. Asif ${ }^{2}$, J. Khalifa ${ }^{3 \star}$, M. Arif ${ }^{4}$ and S. Butt ${ }^{5}$

${ }^{1}$ Bahria University, Karachi Campus, Institute Of Professional Psychology, North Nazimabad Town, Karachi, Pakistan; ${ }^{2}$ Bahria University, Karachi Campus, Institute Of Professional Psychology, Nazimabad Block, Karachi, Pakistan; ${ }^{3}$ Bahria University, Karachi Campus, Institute Of Professional Psychology, DHA Phase, Karachi, Pakistan; ${ }^{4}$ Bahria University, Karachi Campus, Institute Of Professional Psychology, Malir Cantt,Gulshan-e-Umair, Karachi, Pakistan and ${ }^{5}$ Bahria University Karachi Campus, Institute Of Professional Psychology, Karachi, Pakistan, Pakistan ${ }^{*}$ Corresponding author.

Introduction: The phenomenon of road rage has been identified as a key contributor in traffic accidents (James, 2016). This study was conducted to explore the relationship linking dispositional optimism and road rage.

Objectives: It was hypothesized that there is a negative correlation between dispositional optimism and road rage, and that individuals who score low for dispositional optimism are more prone to display road rage as compared to those who score high for dispositional optimism.

Methods: A survey-based quantitative design was employed to establish the correlation. The survey was conducted at shopping malls of Karachi, Pakistan; and online; and the sample consisted of 101 bike riders and 146 car drivers $(\mathrm{N}=247, \mathrm{nb}=101, \mathrm{nc}=146)$ aged 18 and above, recruited via purposive convenient and 
snowball sampling, of which 56 (22.7\%) were female. Participants had a minimum 12 years of education and 1 year of driving or bike riding experience. Professional drivers and bike riders were excluded. Tests administered included the Life Orientation Test-Revised; and the Propensity for Angry Driving Scale. The Statistical Package for Social Sciences (SPSS 22) was used to analyze the data.

Results: The results did not support the hypothesis. Calculation of Pearson's coefficient for the two variables revealed a weak positive relationship $(\mathrm{r}=0.24, \mathrm{p}<0.01)$.

Conclusions: To find solutions for the issue of road rage, research must provide a deeper insight into the variables that perpetuate it. This study contributes to understanding the phenomenon of road rage by exploring its relationship with the variable of dispositional optimism.

\section{Conflict of interest: No}

Keywords: Optimistic Personality; Adult; Road rage; Aggressive Driving

\section{Posttraumatic stress disorder}

\section{EPP0841}

Complex post-traumatic stress disorder in combatants in the view of the eleven edition of the international classification of diseases

\section{A. Haydabrus \\ V.N.Karazin Kharkiv National University, Clinical Neurology, Psychiatry and Narcology., Kharkiv, Ukraine}

Introduction: The increase the number sufferers among military personnel and civilians on the territory of Ukraine of hostilities increases the likelihood of PTSD occurring among the contingent. But at present, in the world no much experience in the diagnosis of cPTSD, that may be in this category of people.

Objectives: The objective of our pilot study, along with the diagnosis of PTSD symptoms, is to use a quality of life questionnaire to evaluate the symptoms of cPTSD.

Methods: Semi-structured interviews were conducted with 26 combatants. We used questionnaire PCL-5 and quality of life (Mezzich and al.).

Results: The average age of patients was 41.3 years, the average length of stay in the army - 19.4 months, the duration of stay in the combat zone -8.2 months. The self-realization of the combatants had a low average level, which further influences the level of adaptation after the end of the service. Physical and psychological well-being were also low, and this indicator to the need for psychological and physical rehabilitation of demobilized soldiers. At the same time, communication with friends and family, performance were at a high enough level, and these were positive factors that influence the adaptation to civilian life.

Conclusions: Based on these results of pilot study, we conclude that combatants has emotional disregulation and negative self-view symptoms, that is part of symptoms of cPTSD and we must consider this in rehabilitation.
Conflict of interest: No

Keywords: complex post-traumatic stress disorder; post-traumatic stress disorder; Adaptation; combatants

\section{Promotion of mental health}

\section{EPP0875}

Mental health promotion strategies for migrants and refugees in Europe: main outcomes, recommendations and lessons learnt from myhealth european project

S. Evangelidou ${ }^{1 *}$, M. Schouler-Ocak ${ }^{2}$, N. Gionakis ${ }^{3}$, N. Movsisyan ${ }^{4}$, A. Qureshi ${ }^{1}$ and F. Collazos ${ }^{1}$

${ }^{1}$ Vall d'Hebron University Hospital / Research Institute, Psychiatry, Barcelona, Spain; ${ }^{2}$ Charité - Universitätsmedizin Berlin, Psychiatrische Universitätsklinik Der Charité, Berlin, Germany; ${ }^{3}$ Syn Eirmos NGO of Social Solidarity Astiki Etairia E, Scientific Coordination, Athens, Greece and ${ }^{4}$ St. Anne's University Hospital Brno, Cardiology, Brno, Czech Republic

${ }^{\star}$ Corresponding author.

Introduction: Upon their arrival in Europe, migrants and refugees often face multiple and highly complex stressors, which risk to have a lasting impact on their mental health. These may be related to traumatisation experiences before, during and after the migration process. Mental health promotion for vulnerable migrants and refugees (VMRs) has been a neglected area of intervention in transcultural psychiatry.

Objectives: MyHealth project (2017-2020) is a European initiative that aims to develop/reinforce models of increased health (and mental health) care access and improved health (and mental health) services for VMRs.

Methods: Four study sites (Barcelona, Berlin, Brno and Athens) were included. Through a participatory and social innovative approach and while engaging VMRs, professionals as well as the general public of the host community, an intercultural needs assessment took place followed by a tools' development phase and a pilot testing of specific tools identified.

Results: Cross-country observations conclude that the coverage of social needs may primarily determine the mental wellbeing of VMRs, and their mental health state can consequently affect their help-seeking behaviours for any experienced physical condition. The prioritization of needs and barriers as viewed by professionals may differ from how are perceived by VMRs. This gap is conditioned by professionals' bias and the intercultural contact between VMRs and host community. To overcome such barriers, specific interventions were piloted at every site (e.g. cultural competence trainings, empowerment of female refugees).

Conclusions: The context-specific results allow to draw outcomes, recommendations and lessons learnt which can inform the agendas of mental health promotion strategies and European mental health policies.

\section{Conflict of interest: No}

Keywords: migrants and refugees and Europe and mental health promotion 


\section{Psychoneuroimmunology}

\section{EPP0888}

\section{Enhanced bacterial translocation in major depressive disorder patients.}

M. Alvarez-Mon ${ }^{1 \star}$, A. Gomez ${ }^{2}$, A. Orozco ${ }^{3}$, G. Lahera ${ }^{4}$, M.D. Sosa ${ }^{2}$, D. Diaz ${ }^{2}$, E. Auba ${ }^{1}$, A. Albillos ${ }^{2}$, J. Monserrat ${ }^{2}$ and M. Alvarez-Mon ${ }^{2}$

${ }^{1}$ University of Navarra Clinic, Department of Psychiatry, Pamplona, Spain; ${ }^{2}$ University of Alcala, Department of Medicine and Medical Specialties, Madrid, Spain; ${ }^{3}$ Hospital Principe de Asturias, Division Of Psychiatry, Madrid, Spain and ${ }^{4}$ University of Alcala, School Of Medicine, Alcalá de Henares, Spain

${ }^{\star}$ Corresponding author.

Introduction: Gut microbial influence on depressive symptoms has recently been described.

Objectives: Investigate gut barrier permeability and bacterial translocation in Major Depressive Patients (MDD) patients.

Methods: We included 22 patients with a Psychiatrist-confirmed diagnosis of MDD, single or recurrent, according to the Diagnostic and Statistical Manual of Mental Disorders criteria (DSM-V). We also included 14 matched healthy controls (HCs). We measured the
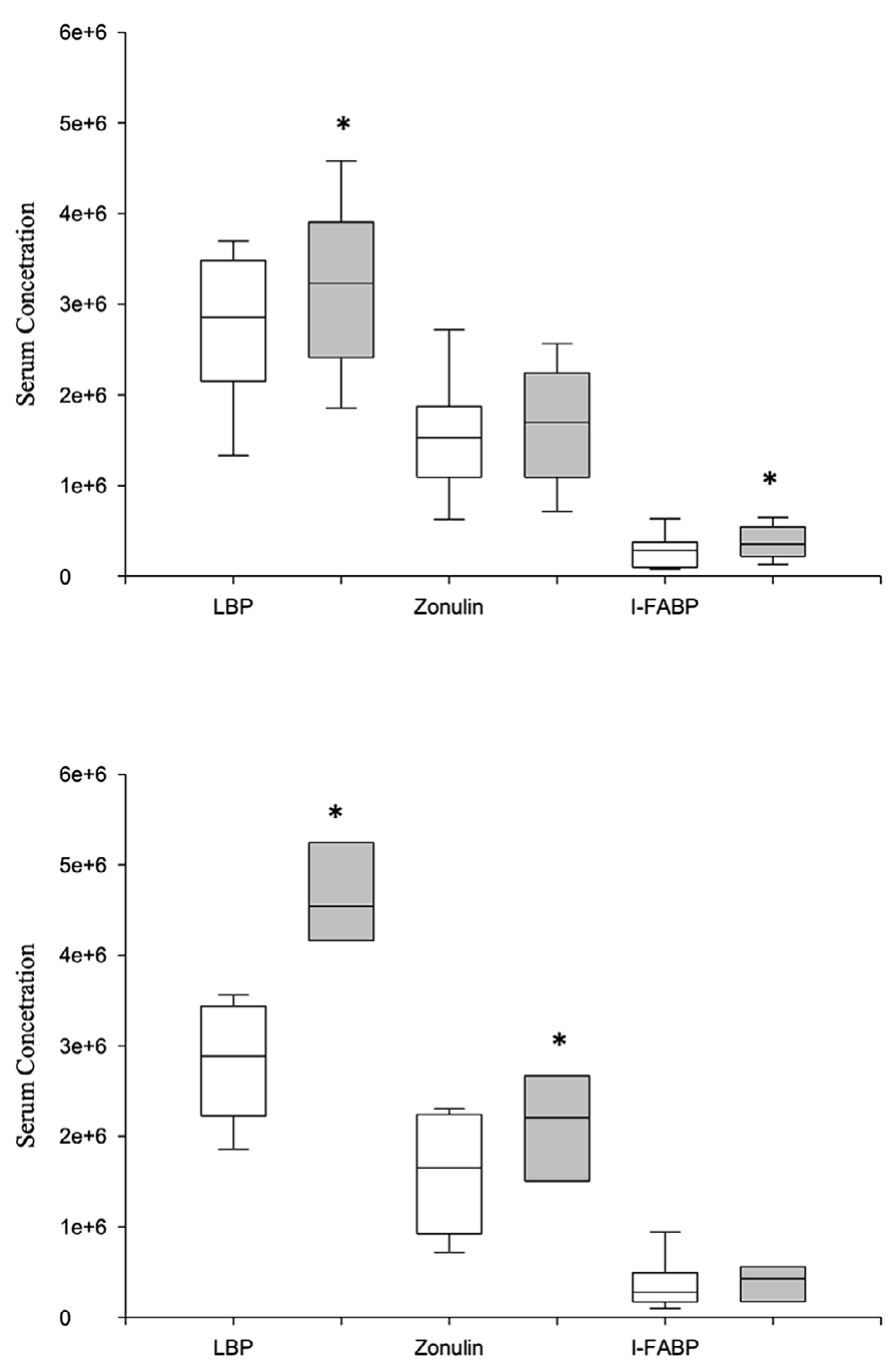

serum levels of LBP, I-FABP and Zonulin to assess bacterial translocation and gut barrier damage.

Results: MDD patients had significantly higher circulating levels of LBP and I-FABP than HC (Figure 1). We found that 7 out of 22 MDD patients had LBP levels higher than the $95^{\text {th }}$ percentile of those found in HCs. High-LBP MDD patients had significantly increased serum Zonulin concentrations compares to those with normal LBP levels (Figure 2).

Conclusions: MDD patients showed increased circulating levels of LBP and I-FABP, suggesting that these patients experience gut barrier damage and increased bacterial translocation.

\section{Conflict of interest: No}

Keywords: bacterial translocation; leaky gut; major depressive disorder; gut-brain axis

\section{EPP0890}

Autoimmunity makes me crazy: psychiatric manifestations of autoimmune encephalitis.

P. Espada-Santos ${ }^{*}$, J. Facucho-Oliveira, M. Albuquerque, M. Costa, D. Esteves-Sousa, L. Mendonça, P. Cintra, J. Tropa and A. Moutinho

Hospital de Cascais Dr. José de Almeida, Departamento De Psiquiatria E Saúde Mental, Alcabideche, Portugal

${ }^{\star}$ Corresponding author.

Introduction: Autoimmune mechanisms causing psychiatric symptoms are increasingly recognized. Autoimmune encephalitis constitutes a group of inflammatory brain diseases associated with autoantibodies against neuronal cell-surface proteins, ion channels or receptors. In $60 \%$ of the cases, psychiatric abnormalities are the most common clinical symptoms. Change in behavior, psychosis, memory and cognitive deficits, abnormal movements, dysautonomia, seizures and decreased level of consciousness are some of the clinical symptoms.

Objectives: To described two clinical cases of autoimmune encephalitis that presented with psychiatric symptoms.

Methods: A 25 years old woman was admitted at Hospital de Cascais with behavioral changes, catatonia, delirium and a recent history of seizures. Anti-NMDAR antibodies were positive in blood samples and cerebrospinal fluid. A 44 years old woman was also admitted Hospital de Cascais with temporo-spacial disorientation, altered speech and behavior, psychomotor agitation and dystonic episodes. Blood samples and cerebrospinal fluid were positive for anti-LGI1 antibodies. In both cases brain MRI were normal and EEG showed in the first case a permanent left fronto-temporal diffuse slowness and in the second case a unspecific diffuse moderated slowness. None of the patients were diagnosed with tumors or previous viral infections.

Results: Both women were treated with corticoids, IV immunoglobulin, cyclophosphamide or with plasmapheresis with small or no clinical improvement. Both responded progressively to Rituximab.

Conclusions: The awareness that autoimmune conditions can cause a broad spectrum of neuropsychiatric symptons, with unusual nature and courses, should prevent misdiagnosis of a primary psychiatric diseases and promote in time identification and management of patients since prompt immunotherapy has been associated with better outcomes.

\section{Conflict of interest: No}


Keywords: Autoimmune encephalitis; autoantibodies; neuropsychiatric symptoms

\section{Psychopharmacology and pharmacoeconomics - Part I}

\section{EPP0913}

\section{Lithium poisoning: a silent killer?}

F. Caetano ${ }^{1 \star}$, A. Samouco ${ }^{2}$, M. Araújo ${ }^{3}$, C. Priscila ${ }^{1}$, P. Frias ${ }^{4}$, M. Barros ${ }^{4}$ and S. Carvalho ${ }^{1}$

${ }^{1}$ Hospital de Magalhães Lemos, Serviço De Internamento B, Porto, Portugal; ${ }^{2}$ Unidade Local de Saúde do Norte Alentejano, Department of Psychiatry and Mental Health, Portalegre, Portugal; ${ }^{3}$ Hospital de Magalhães Lemos, Serviço De Internamento C, Porto, Portugal and ${ }^{4}$ Hospital de Magalhães Lemos, Psychiatry, Porto, Portugal ${ }^{*}$ Corresponding author.

Introduction: Lithium is the most effective treatment in preventing relapse in bipolar I disorder. Despite its benefits, toxicity is common.

Objectives: Review the existing literature on lithium poisoning and illustrate its clinical presentation with a complex clinical case from our practice.

Methods: Literature search and review of the clinical files.

Results: Types of lithium poisoning include acute, acute on chronic and chronic. Symptoms and signs of toxicity differ based upon the total burden of lithium and onset of toxicity. The clinical presentation correlates poorly with blood levels, and includes findings from several organ systems: (i) Gastrointestinal (nausea, vomiting and diarrhea); (ii) Cardiovascular (changes in the ECG, such as prolonged QTc and bradycardia); (iii) Neurological [sluggishness, ataxia, tremor, fasciculations, myoclonic jerks, seizures, extra-pyramidal signs and long term sequelae, including the syndrome of irreversible lithium-effectuated neurotoxicity (SILENT)]. We present the case of a 62-year-old male brought to the emergency department with behavioral alterations, slurred speech and gait abnormalities. During his stay at the hospital he showed multiple signs and symptoms of various stages of lithium poisoning: polyuria, polydipsia, hypotension, bradycardia, acute kidney injury, hypernatremia, nephrogenic diabetes insipidus, encephalopathy and suspected SILENT syndrome.

Conclusions: Close monitoring of patients is crucial to prevent toxicity. With this research we intend to signal red flags and alert clinicians to the clinical course of lithium poisoning and how to treat it.

Conflict of interest: No

Keywords Toxicity; SILENT; Bipolar; lithium

\section{EPP0916}

\section{A chart-based association study of newer}

antidepressant medication use and weight change in a population in Lebanon

J. El-Khoury $^{1 \star}$, A. Beayno ${ }^{1}$, P. Noufi ${ }^{1}$, M. Bejjani ${ }^{2}$, M. Abed Al Ahad $^{3}$, G. Majari ${ }^{4}$ and A. Ahmad ${ }^{1}$

${ }^{1}$ American University of Beirut Medical Center, Psychiatry, Beirut, Lebanon; ${ }^{2}$ American University of Beirut Medical Center, Clinical
Research Institute research Institute, Beirut, Lebanon; ${ }^{3}$ American University of Beirut Medical Center, Clinical Research Institute, Beirut, Lebanon and ${ }^{4}$ American University of Beirut Medical Center,

Radiology, Beirut, Lebanon

${ }^{\star}$ Corresponding author.

Introduction: Antidepressants are amongst the most widely prescribed medications globally and the rate of their use across populations is increasing. Weight changes during antidepressant treatment was found to create severe public health problems, and weight gain was the most commonly reported side effect in the treatment process.

Objectives: To study the association between antidepressant treatment and weight changes in a clinical sample of the Lebanese population.

Methods: A chart-based longitudinal study was used to describe the changes in weight in adult outpatients who started newer-generation antidepressants (SSRIs, SNRIs, Vortioxetine) for a period of six months. The weight of each patient was recorded at initiation of the antidepressant and at any subsequent clinical assessment in the following 6 months.

Results: In a sample of 198 adult outpatients, results revealed that weight did not significantly change from week 1 to week 4 (T1, $\mathrm{p}=0.53$ ), but significantly increased by 0.15 kilograms for each additional week after week 4 (T2, p<.01). Furthermore, there was no difference in weight change between patients on fluoxetine versus those on other anti-depressants $(\mathrm{p}=0.83 / \mathrm{p}=0.72$, for $\mathrm{T} 1$ and T2 respectively). Finally, no difference was found between patients diagnosed with depressive disorders and those with other diagnoses in either time intervals $(\mathrm{p}=0.26 / \mathrm{p}=0.85$, for $\mathrm{T} 1$ and $\mathrm{T} 2$ respectively).

Conclusions: Population-based studies remain the best tool for optimizing psychiatric pharmacotherapy specifically with regards to weight changes. The results of this study advocate for vigilance over weight gain beyond the first 4 weeks of treatment and throughout its course, regardless of external factors.

Disclosure: I have acted as a speaker and consultant for some of the companies that market the antidepressants in the study. These include Lundbeck, Pfizer and Lilly. This excludes all involvement or financing of this specific study. I do not have any other conflict

Keywords Lebanon; Antidepressants; Weight; pharmacology

Psychopharmacology and pharmacoeconomics - Part II

\section{EPP0929}

Applicability of pharmacogenomic testing in the treatment of major depressive disorder

\author{
S. Ramos ${ }^{\star}$, D. Sousa and J. Mendes \\ Unidade Local de Saúde da Guarda, E.P.E., Psychiatry, Guarda, \\ Portugal \\ ${ }^{\star}$ Corresponding author.
}

Introduction: The classical trial-and-error approach to treating major depressive disorder (MDD) provides insufficient results in clinical practice. Pharmacogenomic-guided selection of antidepressant medication has been proposed to enhance treatment response and reduce adverse effects, despite mixed results. 
Objectives: To ascertain the applicability of pharmacogenomic testing in adult patients with MDD.

Methods: We performed a rapid overview of reviews and metaanalyses from PubMed through 2010 to September 2019.

Results: Four studies met the inclusion criteria. The reviews reported mixed findings, compiled mostly from low quality primary studies affected by conflicts of interest. A meta-analysis presented a risk ratio (RR) for response of 1.36 in favor of guided treatment and an improved number needed to treat (NNT) in nonblinded primary studies compared to randomized controlled trials (NNT 6vs.8, respectively). Certain pharmacogenomics tools appear to improve short-term remission rates in women in their mid-40 with few comorbidities. A systematic review comparing GeneSightguided care to treatment as usual found improved response to depression treatment and greater improvements in measurements of depression. Finally, a systematic review on the predictive effect of CYP2D6 and CYP2C19 testing for antidepressant response or adverse drug reactions found mixed results, suggesting that CYP2D6 and CYP2C19 testing may predict response in certain individuals. However, the review failed to inform whether it translated to improved or safer clinical outcomes.

Conclusions: Available evidence on pharmacogenomic testing remains insufficient to support routine pharmacogenetic guided therapy for MDD. Robust and well-designed studies are necessary to evaluate clinical efficacy, tolerability and cost effectiveness of pharmacogenomic testing.

Conflict of interest: No

Keywords major depressive disorder; Pharmacogenomics

\section{EPP0936}

\section{A second generation of neuroleptic malignant syndrome - a case report \\ C. Vieira Mendes ${ }^{\star}$ and P. Ferreira \\ ULSAM, Psiquiatria E Saúde Mental, viana do castelo, Portugal ${ }^{*}$ Corresponding author.}

Introduction: The big new that second generation antipsychotics (SGA) brought when released was the absence of neuroleptic malignant syndrome (NMS). Unfortunately, although less frequent, NMS remains present. It has been reported atypical cases, misrepresenting the original nosological entity.

Objectives: Report a clinical case of an atypical NMS after monthly injectable paliperidone palmitate.

Methods: A 40-year old man, diagnosed with Squizophrenia and medicated with injectable paliperidone palmitate $150 \mathrm{mg}$ monthly for 4 years, presented at the emergency department after being met by his parents in the morning praying on his knees, telling he felt he was going to die (3 days after the antipsychotic medication). At the hospital, he complained about muscular pain and weakness, but no rigidity. Blood tests revealed acute renal failure, increased liver enzymes, rhabdomyolysis (creatine phosphokinase $>85340 \mathrm{UI} / \mathrm{L}$ ) and metabolic acidosis. Because of a short period of prostration, he had a brain computed tomography, with no relevant finding. At the Day 3 , he showed hyperthermia $\left(37,9^{\circ} \mathrm{C}\right)$, increased heart rate and arterial hypertension, tachypnea, leukocytosis (but no increased C-reactive protein) and worsening renal failure and CK levels.

Results: It was assumed a NMS, with atypical characteristics, after infectious causes excluded. Only supportive treatment was performed, with no possibility of antipsychotic agent removal.
Conclusions: These rare heterogeneous cases of NMS with SGA need a careful approach. Appart from clinical issues, this term should be revised for being no more suitable, since the term "neuroleptic" is decaying, and the clinical is no longer as lethal as with first generation antipsychotics.

\section{Conflict of interest: No}

Keywords antipsychotics; malignant neuroleptic syndrome; second generation

\section{Psychosurgery \& stimulation methods (ECT, TMS, VNS, DBS)}

\section{EPP0948}

\section{Neuroplastic effects of electroconvulsive therapy may be mediated by reduced IL- 6 levels}

J.-B. Belge ${ }^{1 \star}$, L. Van Diermen ${ }^{1}$, P. Sienaert ${ }^{2}$, K. Vansteelandt ${ }^{2}$, P. Parizel ${ }^{1}$, B. Sabbe ${ }^{3}$, E. Constant ${ }^{4}$, P. De Timary ${ }^{4}$, M. Morrens ${ }^{1}$, V. Coppens ${ }^{1}$, D. Schrijvers ${ }^{3}$ and P. Vaneijndhoven ${ }^{5}$

${ }^{1}$ Antwerp University, Adult Psychiatry, Antwerp, Belgium; ${ }^{2}$ Katholieke Universiteit Leuven, Adult Psychiatry, Leuven, Belgium; ${ }^{3}$ Antwerp University, Adult Psychiatry, antwerp, Belgium; ${ }^{4}$ Université Catholique de Louvain, Adult Psychiatry, Brussels, Belgium and ${ }^{5}$ Radboud University, Adult Psychiatry, Nijmegen, Netherlands ${ }^{*}$ Corresponding author.

Introduction: ECT has a powerful immunomodulatory function. A way to bridge the gap between immunomodulation and ECTs' therapeutic efficacity in depression could be ECT-induced neuroplasticity, as proinflammatory cytokines like IL-6 and TNFalpha tend to decrease after an ECT cure and are implicated in hippocampal neurogenesis, important for the antidepressant effect. I Objectives: To examine the immunomodulatory effect of ECT and analyze whether the hippocampal volumetric changes in depressed patients treated with ECT are related to changes in IL- 6 and TNFa levels.

Methods: Plasma levels of IL-6 and TNFa as hippocampal volume by magnetic resonance imaging were analyzed before and after an acute ECT course in respectively a total sample of 62 and a sub sample of 14 patients. Mood changes were assessed 1 week before and after ECT with the Hamilton Depression Rating Scale.

Results: Participants showed a significant improvement of mood after ECT $(\mathrm{p}<0.0001)$. We observed a significant decrease of IL-6 levels $(p<0.05)$. A significant volume increase of the hippocampus was found $(\mathrm{p}<0.001)$. We did notice a significant negative correlation between IL- 6 difference scores and the percentage change in hippocampal volumes $(\mathrm{r}=-0.8, \mathrm{p}<0.01)$. We found no significant correlation between changes in mood and volumetric nor inflammatory variables.

Conclusions: Our findings support the immunomodulatory effects of ECT and an association between a volumetric increase of the hippocampus and lower levels of IL-6. These findings suggest that ECTs neuroplastic effect may be mediated by its immunomodulating functions, but the clinical relevance of this biological phenomenon remains uncertain.

\section{Conflict of interest: No}

Keywords Major Depression Disorder; Interleukin 6; Electroconvulsive Therapy; Hippocampus 


\section{Rehabilitation and psychoeducation - Part I}

\section{EPP0989}

\section{Physical exercise interventions in first-episode psychosis}

R. Gasparinho*, N. Fernandes, N. Santos, M. Martins, A. Alho, L. Ferreira and A. Ribeirinho

Hospital Distrital de Santarém, Psychiatry and Mental Health, Santarém, Portugal

${ }^{*}$ Corresponding author.

Introduction: Patients with long-term schizophrenia are known to display lower levels of physical activity than general population. Usually this is linked to higher rates of obesity, cardio-metabolic diseases and premature mortality. Negative symptoms and cognitive deficits leading to impaired functionality are also a hallmark of long-term schizophrenia. Most of these features are already present in patients with a first-episode psychosis (FEP). In these patients lifestyle interventions, such as physical exercise (PE) may have an important role in health promotion, disease prevention, and in symptomatic and functional recovery.

Objectives: To review the effects of interventions focusing on physical exercise in patients with first-episode psychosis.

Methods: Pubmed search with MeSH terms: "Exercise", "Schizophrenia", "Psychotic Disorders". Search was restricted to articles written in English, temporal limitation of 10 years. Total of 261 results; 19 articles selected.

Results: PE is a critical component of lifestyle interventions, since it is associated with decreases in all-cause morbidity and mortality. $\mathrm{PE}$ as adjunctive interventions in patients with FEP have shown positive effects which include improving quality of life and body image, prevention of weight gain, reduction of cardiometabolic risk. Conclusions: Early lifestyle interventions such as PE in patients with a FEP are a promising field, but large randomized controled trials are lacking to define both feasibility and overall short- and long-term benefits.

Conflict of interest: No

Keywords: physical exercise; first-episode psychosis; Non-

pharmacological interventions; rehabilitation

\section{Schizophrenia and other psychotic disorders - Part I}

\section{EPP1018}

\section{When psychosis faces twitter.}

M. Alvarez-Mon ${ }^{1 \star}$, M. Llavero-Valero ${ }^{2}$, R. Sanchez-Bayona ${ }^{1}$, V. Pereira-Sanchez ${ }^{3}$, M. Vallejo-Valdivielso ${ }^{4}$, J. Monserrat ${ }^{5}$, G. Lahera ${ }^{6}$, A. Asunsolo Del Barco ${ }^{5}$ and M. Alvarez-Mon ${ }^{5}$

${ }^{1}$ University of Navarra Clinic, Department of Psychiatry, Pamplona, Spain; ${ }^{2}$ University of Navarra Clinic, Department of Endocrinology, Madrid, Spain; ${ }^{3}$ New York University, Langone Child Study Center, New York, United States of America; ${ }^{4}$ University of Navarra Clinic, Child \& Adolescent Psychiatry Unit, Department of Psychiatry and Clinical Psychology, Pamplona, Spain; ${ }^{5}$ University of Alcala,

Department of Medicine and Medical Specialties, Madrid, Spain and

${ }^{6}$ University of Alcala, School Of Medicine, Alcalá de Henares, Spain

${ }^{\star}$ Corresponding author.
Introduction: Psychotic disorders are among the world's leading causes of disability. There is evidence of persisting stigma that leads to negative stereotyping and to discriminatory behaviors.

Objectives: Analyze the content of tweets referring to Psychosis. Methods: We focused on searching over a period of eight consecutive days in 2018 for tweets with the following hashtags: \#psychosis, \#psychotic, \#schizophrenia, \#schizophrenic. Each tweet's content was rated as non-medical (testimonies, health care products, advocacy or misuse) or medical (included a reference to the illness's diagnosis, treatment, prognosis, or prevention). non-medical tweets were classified as positive or pejorative; we also evaluated the appropriateness of the medical content.

Results: We analyzed a total of 1029 tweets: 636 (61.81\%) were classified as non-medical and $393(38.19 \%)$ as non-medical. The medical content showed high scientific appropriateness and focused on promoting a healthy lifestyle $(67.5 \%)$, whereas non-medical content focused on advocacy and testimonies (63.2\%). However, misuse was present (10\%), reflecting the bad social habit of using "schizophrenia" or "psychosis" to refer to "madness" (Table 1).

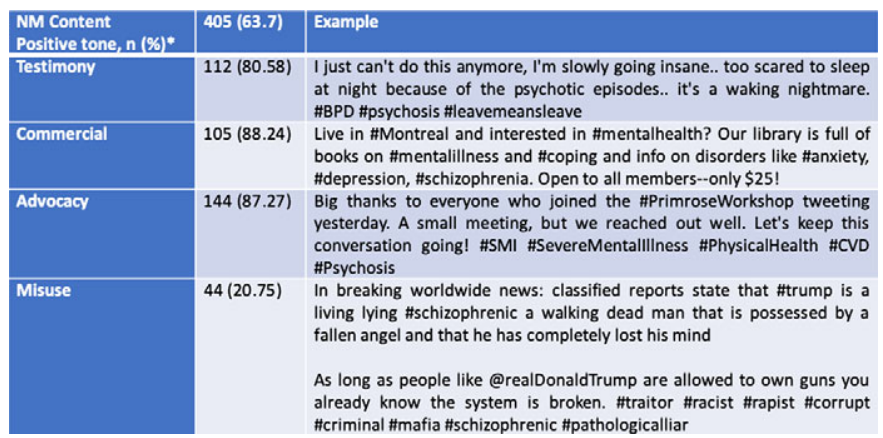

Conclusions: Twitter may be an adequate tool for antistigma campaigns and promotion of healthy habits.

Conflict of interest: No

Keywords: schizoprenia; psychosis; Twitter; Stigma

Schizophrenia and other psychotic disorders - Part IV

\section{EPP1052}

High doses of aripiprazol once-monthly in the treatment of patients with severe schizophrenia. A 36-month follow-up

J.J. Fernandez-Miranda ${ }^{1 *}$ and S. Diaz-Fernandez ${ }^{2}$

${ }^{1}$ AGC SM-V-HUCAB-SESPASTURIAN MENTAL HEALTH

SERVICE, Centro De Tratamiento Integral, GIJON, Spain and ${ }^{2}$ AGC-S MENTAL-V-SESPAASTURIAN MENTAL HEALTH SERVICE, Centro De Tratamiento Integral, GIJON, Spain

${ }^{*}$ Corresponding author.

Introduction: The current study explores whether high-dose therapy with a SGA ILD may be of benefit in patients with schizophrenia inadequately controlled on standard dose.

Objectives: To evaluate treatment retention, effectiveness and tolerability of high doses of aripiprazol once-monthly long-acting (AM) in the treatment of patients with severe (Clinical Global Impression-Severity (CGI-S) of 5 and over) schizophrenia. 
Methods: 36-month prospective, observational study of patients with severe schizophrenia who underwent treatment with $600 \mathrm{mg}$ and over of aripiprazole once-monthly $(\mathrm{AM})(\mathrm{N}=30)$ in order to get clinical stabilization due to lack of effectiveness. Assessment included the CGI-S, the WHO Disability Assessment Schedule (WHO-DAS), laboratory tests, weight, adverse effects and reasons for treatment discontinuation. Hospital admissions in the previous three years and during the follow-up were recorded.

Results: The average doses was 780 (120) mg/28 days. Tolerability was good, with few side effects reported and biological parameters alterations. There were two discharges due to side effects and one due to lack of effectiveness. Weight and prolactin levels decrease, but not significantly. After three years, CGI-S $(\mathrm{p}<0.01)$ and WHODAS in the four areas (self-care $\mathrm{p}<0.005$; occupational $\mathrm{p}<0.01$; family $\mathrm{p}<0.01$; social impairment $\mathrm{p}<0.05$ ) decreased. There were significantly less hospital admissions than during the previous 36 months: $1.3(1.1)$ vs. $0.3(0.2)(\mathrm{p}<0.001)$.

Conclusions: Tolerability of high doses of aripiprazol once-monthly was very good, being useful in improving treatment adherence in patients with severe schizophrenia, and helping this way to get clinical stabilization and better functioning.

Conflict of interest: No

Keywords: schizophrenia; antipsychotics; treatment outcomes; doses

\section{EPP1056}

\section{Clinical features of late-onset schizophrenia: a case report}

\section{Fernandes Santos*, C. Miranda and A.B. Medeiros}

Hospital Garcia de Orta, Department of Psychiatry and Mental Health, Almada, Portugal

${ }^{\star}$ Corresponding author.

Introduction: Manfred Bleuler introduced the term "late-onset schizophrenia" (LOS) in 1943, after accompanying 126 patients whose condition started between the ages of 40 and 60 , with symptoms fundamentally similar to the classic form, in the absence of organic brain disease. LOS presents clinical characteristics that distinguish it from schizophrenia in early life, concerning symptom profile, etiological and epidemiological aspects, risk factors and therapeutic response. Objectives: Description of a clinical case of LOS, followed by a review of its differentiating clinical features.

Methods: Non-systematic review of literature indexed to PubMed/ MEDLINE up to 2019, using the terms "schizophrenia" and "lateonset". To describe the clinical case, medical records were used.

Results: A 58-year-old widow woman is admitted to our inpatient unit for a clinical picture with 13 years of evolution, characterized by persecutory and mystical-religious delusions, auditory hallucinations, as third-person accusatory voices, and somatic passivity phenomena, with high behavioural impact. No cognitive deficits were evidenced and the patient remained globally functional. Diagnostic tests did not reveal any alterations. The patient initiated high-dose antipsychotic therapy with partial response. LOS is associated with a higher ratio of women and better occupational and marital histories. Negative symptoms and cognitive alterations are not prominent, although LOS may predict dementia. Risk factors include genetics, menopause, vascular/ neurodegenerative lesions, sensory deficits and age per se. Therapeutic response to antipsychotics is usually good, although postmenopausal women need higher doses and show worse prognosis.
Conclusions: There are clinical, etiological, epidemiological and therapeutic particularities that make LOS a valid subtype with clinical utility, as illustrated in our case.

Conflict of interest: No

Keywords: schizophrenia; psychosis; late-onset

Schizophrenia and other psychotic disorders - Part IX

\section{EPP1063}

Functioning in schizophrenia: a worldwide delphi study L. Nuño $^{1 \star}$, M. Barrios ${ }^{2}$, E. Rojo ${ }^{3}$, J. Gómez-Benito ${ }^{2}$ and G. Guilera ${ }^{2}$

${ }^{1}$ Hospital Clínic de Barcelona, Addictions Unit. Psychiatry and Psychology Service, Barcelona, Spain; ${ }^{2}$ University of Barcelona, Department of Social Psychology and Quantitative Psychology, Barcelona, Spain and ${ }^{3}$ Hospital Benito Menni, Casm, Sisters Hospitallers, Sant Boi de Llobregat, Spain ${ }^{*}$ Corresponding author.

Introduction: The International Classification of Functioning, Disability, and Health (ICF) Comprehensive Core Set for schizophrenia (CS-SZ) is a shortlist of ICF categories that are relevant for describing the functioning and disability of persons suffering from schizophrenia.

Objectives: This study aims to assess the common aspects and the differences regarding the relevance of the CS-SZ categories among health professionals experienced in schizophrenia treatment that belong to the six World Health Organization (WHO) regions (i.e. African, The Americas, Eastern Mediterranean, European, South East Asia, and Western Pacific).

Methods: In a three-round survey using the Delphi technique, 790 health professionals (i.e., psychiatrists, psychologists, nurses, occupational therapists, social workers, and physiotherapists) from 85 countries representing all six WHO regions were asked about the problems they commonly encounter in patients with schizophrenia. Their responses were linked to ICF categories and those that reached consensus $(\geq 75 \%)$ were selected.

Results: A total of 138 second-level ICF categories were selected, and 79 were common across all six WHO regions: 13 Body functions, 1 Body Structures, 33 Activities and Participation, and 32 Environmental factors. Most of the ICF categories of the CS-SZ $(78 \%)$ were also common across all six WHO regions. The range of concordance among WHO regions ranged from $79.4 \%$ (e.g., Americas and Europe) to $88.7 \%$ (i.e., Africa and Eastern Mediterranean). Conclusions: A large number of problems describing functioning and disability in individuals with schizophrenia are common in all six WHO regions. Moreover, data show that ICF categories from the CS-SZ are mostly accepted across all WHO regions.

Conflict of interest: No

Keywords: functioning; schizophrenia; Delphi study; WHO regions

\section{EPP1064}

Functioning in schizophrenia from the perspective of occupational therapists: an international delphi study

L. Nuño ${ }^{1 *}$, M. Barrios ${ }^{2}$, E. Rojo ${ }^{3}$, J. Gómez-Benito ${ }^{2}$ and G. Guilera ${ }^{2}$ 
${ }^{1}$ Hospital Clínic de Barcelona, Addictions Unit. Psychiatry and Psychology Service, Barcelona, Spain; ${ }^{2}$ University of Barcelona, Department of Social Psychology and Quantitative Psychology, Barcelona, Spain and ${ }^{3}$ Hospital Benito Menni, Casm, Sisters Hospitallers, Sant Boi de Llobregat, Spain ${ }^{*}$ Corresponding author.

Introduction: The International Classification of Functioning, Disability, and Health (ICF) offers a theoretical framework for assessing functioning and disability. The ICF Core Sets for schizophrenia are a list of ICF categories describing the most common problems in functioning of persons affected by this health condition. Objectives: This study aimed to identify the problems occupational therapists (OT) commonly encounter when treating individuals with schizophrenia and to validate these Core Sets from their perspective. Methods: In a three-round survey using the Delphi technique, OTs experienced in the treatment of individuals with schizophrenia were asked about the patients' problems and environmental factors they considered more relevant when treating this population. Two trained health professionals linked responses to the ICF; statistical measure of agreement and kappa coefficient was calculated.

Results: A total of 92 OTs from 29 countries covering the six World Health Organization regions named 2527 meaningful concepts in the first-round. After the linking process, 121 ICF categories and 31 Personal factors were presented to the expert panel. Consensus (agreement $\geq 75 \%$ ) was reached for 97 ICF categories and 27 Personal factors. All the categories present in the Brief ICF Core Sets for schizophrenia and 89 of the 97 categories represented at the Comprehensive version reached consensus.

Conclusions: The ICF Core Sets for schizophrenia were validated from the perspective of OTs and may offer a useful tool to be used by them at clinical practice, as cover a wide variety of problems that they deal with in their interventions with persons with schizophrenia.

Conflict of interest: No

Keywords: Occupational therapy; schizophrenia; functioning; Delphi study

\section{Schizophrenia and other psychotic disorders - Part V}

\section{EPP1083}

\section{Let the games begin}

J. Gonçalves Cerejeira*, I.D.L.M. Santos Carrasco, C. Capella, E. Rodríguez, M. Queipo De Llano, A. Gonzaga, G. Guerra, M. De Lorenzo, M. Gómez, N. De Uribe and O. Santiago

Hospital Clinic of Valladolid, Psychiatry, Valladolid, Spain ${ }^{\star}$ Corresponding author.

Introduction: It is widely known that schizophrenia is associated with some brain structural abnormalities such as lateral ventricular enlargement or total brain volume reduction. However, less frequent abnormalities such as ectopic grey matter have also been described. Periventricular heterotopia is the presence of grey matter foci adjacent to lateral ventricles caused by a neuronal migration abnormality during the neurodevelopment. This alteration was found occasionally in neuropsychiatric disorders such as psychosis, depression, anxiety and autism.

Objectives: To present a case of a first-psychotic episode in a patient with grey matter periventricular ectopia and to review the literature about brain structural abnormalities associated with psychosis.
Methods: Case report and literature review.

Results: We report a case of a 28 -year-old man, with no psychiatric nor substance abuse history. Both, mother and aunt, were already diagnosed of schizophrenia. Patient visited our psychiatric emergency department with auditory hallucinations, persecutory and referential delusions, confusion and psychomotor slowing. His family reported that the previous week the patient remained locked in his room playing videogames and accused them several times of being androids who wanted to harm him. Physical exam, blood analysis and EEG: no significant alterations. Brain MRI: bilateral foci of subependymal heterotopic gray matter adjacent to lateral ventricles in FLAIR sequence. We started treatment with paliperidone with clinical improvement in three weeks.

Conclusions: A few studies have suggested that periventricular heterotopia can be found in neuropsychiatric disorders such as psychosis and our case report support these findings.

\section{Conflict of interest: No}

Keywords: Periventricular heterotopia; first-psychotic episode

\section{Schizophrenia and other psychotic disorders - Part VI}

\section{EPP1085}

The study of needs and burden associated with severe mental illness in azerbaijan.

\section{J. Ismayilova}

National Mental Health Center of the Ministry of Health, Consulting and Treatment, Baku, Azerbaijan

Introduction: In Azerbaijan the same as in the other countries of Central and Eastern Europe mental health services remain to be resource-directed rather than need-directed. Therefore multitude of unmet needs in people with serious mental illness (SMI) is unarticulated and under-recognized by healthcare providers. At the same time mental health reform focusing on the transition from institutional towards community-based services requires better understanding of psychiatric, physical and psychosocial needs of mental health service users.

Objectives: The aim of this study was to assess met and unmet needs in patients with SMI and their family members, as well as their contribution to the burden of illness and satisfaction with services.

Methods: The study involved 50 patients with SMI and 50 family members who were applying for outpatient treatment to the National Mental Health Centre in Baku. Camberwell Assessment of Need Scale (CANSAS) and Verona Services Satisfaction Scale(VSSS-EU) were administered to both patients and family members, while Involvement Evaluation Questionnaire was used in family members only.

Results: Patients and their family members reported very similar need profiles irrespective of age, gender, educational and marital status. A significant burden in families caring for people with SMI was associated with support provided by mental health services. Despite the moderate level of satisfaction with care in patients and relatives, many services were unavailable or unaffordable for them. Conclusions: The implication of the study results may be used for the future planning and development of mental health service in Azerbaijan to achieve better outcomes in terms of addressing patients and their families' most prominent needs.

Conflict of interest: No

Keywords: schizophrenia; Burden; Severe mental illness; needs 
Schizophrenia and other psychotic disorders - Part VII

\section{EPP1103}

Impact of a first psychosis episode program on functional variables after two years of follow-up

M. Matilde ${ }^{1 \star}$, E. García De Jalón ${ }^{2}$, M. Otero ${ }^{3}$, N. Pêreda ${ }^{4}$ and M.C. Ariz ${ }^{5}$

${ }^{1}$ Servicio Navarro de Salud, Salud Mental Primeros Episodios, Pamplona, Spain; ${ }^{2}$ Complejo Hospitalario de Navarra, Servicio De Psiquiatría, Pamplona, Spain; ${ }^{3}$ OSASUMBIDEA, Salud Mental Primeros Episodios, Pamplona, Spain; ${ }^{4}$ osasunbidea, Salud Mental Primeros Episodios, Pamplona, Spain and ${ }^{5}$ Servicio Navarro de Salud, Servicio De Psiquiatría. Primeros Episodios., Pamplona, Spain ${ }^{\star}$ Corresponding author.

Introduction: Early Intervention Services for Early-Phase Psychosis have shown efficay and effectiveness (Correl C, JAMA). In Pamplona, Spain, there is an Early Intervention Program that has been providing assistance for First Psychotic Patients for the last two years.

Objectives: The aim of this study is to analyze the longitudinal effects of the different interventions in several functional variables: GAF, occupational state, CGI-CogS, QLS, SOFAS and WHODAS II scales applied to 204 patients during two years of follow-up.

Methods: We apply a standardized evaluation protocol to every patient at different times: premorbid, initial time, and every three months during follow up. The QLS, SOFAS and WHODAS II scales are applied at 6,12, 18 and 24 months of follow-up. We analyze the data with the SPSS statistical program to see the results in these variables.

Results: Occupational state: regarding baseline, patients with normalized jobs/studies are $60.7 \%$. This percentage persist during months 6, 12 and 18, but decline at month 24 (lower sample size) The GAF scale shows a decline during the first six months, but tends to reach the premorbid levels after a year of treatment. The WHODAS II results don't show any improvement until month 24 We haven't found any improvent in the SOFAS scale. The QLS results shows a progresional improvent in every subscale during the time of follow-up

Conclusions: The Early Intervention Services improve, not only psichopatological dimensions but also functional factors, what is important for the hole recovery of First Psychotic Patients.

Conflict of interest: No

Schizophrenia and other psychotic disorders - Part VIII

\section{EPP1106}

Acylcarnitines and amino acids change with schizophrenia

I. Mednova ${ }^{1 \star}$, M. Kassakin ${ }^{2}$, A. Chernonosov ${ }^{2}$, E. Kornetova ${ }^{3}$, A. Semke ${ }^{3}$, N. Bokhan ${ }^{4}$, V. Koval ${ }^{2}$ and S. Ivanova ${ }^{1}$

${ }^{1}$ Mental Health Research Institute, Tomsk National Research Medical Center of the Russian Academy of Sciences, Laboratory Of Molecular Genetics and Biochemistry, Tomsk, Russian Federation;
${ }^{2}$ Institute of Chemical Biology and Fundamental Medicine, Siberian Branch of the Russian Academy of Sciences, Core Facility Of Mass Spectrometric Analysis, Novosibirsk, Russian Federation; ${ }^{3}$ Mental Health Research Institute, Tomsk National Research Medical Center, Russian Academy of Sciences, Department of Endogenous Disorders, Tomsk, Russian Federation and ${ }^{4}$ Mental Health Research Institute, Tomsk National Research Medical Center of the Russian Academy of Sciences, Addictive States Department, Tomsk, Russian Federation ${ }^{\star}$ Corresponding author.

Introduction: Various factors and mechanisms, such as mitochondrial dysfunction, inflammation, lipid oxidation, DNA damage, oxidative stress, and apoptosis, may be involved in the pathogenesis of schizophrenia. These disturbances can be reflected in metabolomic profiles in patients with schizophrenia. Amino acids and acylcarnitines have an important role in the pathways mentioned above and may become potential biomarkers for identifying features in subjects with schizophrenia.

Objectives: Objective of this research is to study serum amino acids and acylcarnitine levels in schizophrenia.

Methods: Analysis of the 13 amino acid and 30 acylcarnitine was performed on an Agilent 6410QQQ tandem mass spectrometer (Agilent Technologies, USA) using the MassChrom ${ }^{\circledast 5} 5000$ test system (Chromsystems, Germany) using isotope-labeled standards for the quantification of acylcarnitines in dry serum stains. Statistical processing of findings was conducted with the use of the scripting language R using the packages "dplyr", "caret”, "factoextra", "gglplot2" and others.

Results: The study included 37 subjects with schizophrenia. Healthy controls $(n=36)$ included an age- and sex-matched cohort without known disease symptoms. We found a significant decrease in the concentration of valine, aspartate, citrulline, glycine, arginine, glutamate and ornithine as well as long-chain acylcarnitines (C14-C18) and C5:1 and increase C4DC in patients with schizophrenia in comparison to the control group. No other significant differences were established.

Conclusions: The obtained results allow us to consider amino acids and acylcarnitines, as potential biomarkers schizophrenia. This work is supported by RSF grant N 18-15-00011

Disclosure: This work is supported by RSF grant N 18-15-00011

Keywords: Acylcarnitine; Amino acid; schizophrenia; Metabolomics

\section{EPP1107}

"Every breath you take...I'll be watching you" celebrities' stalking and its connection with mental illness

\section{P. Melo-Ribeiro ${ }^{1 \star}$, F. Santos ${ }^{2}$, S. Batista ${ }^{3}$ and M. Mota-Oliveira ${ }^{3}$ \\ ${ }^{1}$ Centro Hospitalar Universitário do Algarve, Departamento De Psiquiatria E Saúde Mental De Faro, Faro, Portugal; ${ }^{2}$ Centro Hospitalar Universitário do Algarve, Psychiatry, Faro, Portugal and ${ }^{3}$ Centro Hospitalar Universitário do Algarve, Departamento De Psiquiatria E Saúde Mental - Faro, Faro, Portugal \\ ${ }^{\star}$ Corresponding author.}

Introduction: Public figures and celebrities are at a greater risk of being victims of stalking. Perpetrators are often diagnosed with mental disorders. Personality traits and pre-attack signs should be better understood given the higher risk of violent outcomes. 
Objectives: To review the literature on stalking behavior and to present a case report illustrating this issue.

Methods: Patients' data were collected from the database SClinico and literature review was performed using Pubmed.

Results: Although stalking behaviour is more frequently perpetrated by males, female stalkers tend to be younger. They also tend to be single, educated, employed and often have a mental disorder (most commonly delusional disorder and personality disorders). These situations present a forensic challenge regarding their legal consequences. We present the case of S., 49 years old, female, single, with a college degree, with an erotomaniac delusion with a musician, after attending one of his concerts, and her mother, B., 68 years old, also with a delusional disorder induced by S. (Folie à deux). Both engaged in stalking behaviours which intensified during a period of 5 years.

Conclusions: Female stalkers appear to have different characteristics than male ones. Despite this, the danger of violent attacks still exists. Therefore, in order to prevent these attacks, we should better understand the pre-attack signs, especially in an era of increased use of social media networks. A forensic psychiatric evaluation maintains an important role given the frequency of mental illness in these cases.

\section{Conflict of interest: No}

Keywords: stalking; Personality disorders; mental illness; Delusional disorder

\section{EPP1109}

\section{The role of the gut microbiome in schizophrenia: current evidence}

\section{A. Miguel ${ }^{\star}$, M. Basto and E. Mendes}

Centro Hospitalar Vila Nova de Gaia/Espinho, Serviço De Psiquiatria, Vila Nova de Gaia, Portugal

${ }^{\star}$ Corresponding author.

Introduction: In schizophrenia, chronic inflammation and oxidative stress occur at an earlier age. The gut microbiome is a population of microbes that form a super-organism with which we have co-evolved. It regulates many metabolic processes, stimulates immune maturation, defends against pathogens and stabilizes the gut barrier. Gut dysbiosis may underlie the pro-inflammatory milieu that is implicated in schizophrenia. Through it's manipulation, the microbiome may have an ability to improve psychiatric and cognitive well being. Given this recent knowledge, the concept of "psychobiotics" has emerged in recent years.

Objectives: This systematic review aims to evaluate the most recent evidence regarding the gut microbiome in populations with schizophrenia.

Methods: We are conducting a PubMed search with the following string: "Microbiome and (schizophrenia OR psychosis)". All abstracts are examined by two investigators separately and selected according to specific criteria.

Results: In the reviewed literature, six genera of bacteria seem to be increased in schizophrenia, while five other were decreased. Symptom severity was positively correlated with three genera and negatively with one. One study reported Ruminococcaceae to be inversely associated with severity of negative symptoms. Lactic Acid bacteria were relatively more abundant in serious mental illness and associated with worse outcomes.

Conclusions: Although our research is still ongoing, the potential clinical implications of gut microbiome modulation are enthusiastic.
It is common to find patients who are resistant to pharmacologic treatment and experience many adverse effects. Modulation of the gut microbiota may be a tractable strategy for developing novel treatments without major side effects or high costs.

Conflict of interest: No

Keywords: schizophrenia; Gut; Microbiome; psychosis

\section{EPP1115}

Falling in love with whom? A case report and brief literature review of coexistence of de clerambault and fregoli syndromes.

N. Moura ${ }^{1 \star}$, L. Santana ${ }^{2}$, J. Facucho ${ }^{3}$, S. Domingues ${ }^{4}$, D. EstevesSousa $^{5}$ and H. Canas-Simião ${ }^{2}$

${ }^{1}$ Centro Hospitalar Lisboa Ocidental, Psychiatry, Lisboa, Portugal;

${ }^{2}$ Centro Hospitalar Lisboa Ocidental, Departamento De Psiquiatria E Saúde Mental, Lisbon, Portugal; ${ }^{3}$ Hospital de Cascais Dr. José de Almeida, Psychiatry, Alcabideche, Portugal; ${ }^{4}$ Centro Hospitalar Médio Tejo, Psychiatry, Tomar, Portugal and ${ }^{5}$ Hospital de Cascais,

Department of Psychiatry, Cascais, Portugal

${ }^{\star}$ Corresponding author.

Introduction: Although originally present in the description by Courbon and Fail, the association of Fregoli delusion with Erotomania has rarely been reported

Objectives: Describe a case report of first-episode psychosis presenting with Fregoli syndrome and erotomania, prompting a literature review.

Methods: Clinical information was obtained by interview and electronic record, after obtaining informed consent. A semistructured review of Fregoli syndrome and Erotomania was conducted on Pubmed.

Results: A 51-year-old male, with no previous psychiatric history, married, working as an insurance broker, went to the emergency department by his wife's initiative, after 4-year history of psychotic symptoms. Mental state examination revealed paranoid delusions of erotomania, persecution and Fregoli syndrome, without insight. He claimed to see the woman who he believes is in love with him wherever he goes, even when abroad on business trips, and she frequently changes her appearance to get close to him without being found. Although his marriage had been affected, no relevant functional impact seemed present. Brain imaging (CT) and analytical study showed no relevant findings. Risperidone $3 \mathrm{mg}$-qd was started with discharge to outpatient assertive care.

Conclusions: The accurate relationship between these syndromes and their basic etiologies remains unclear. Although earlier literature suggested a role for pimozide in the treatment of delusional syndromes, atypical antipsychotics are being preferred given their more advantageous side-effect profile.

\section{Conflict of interest: No}

\section{EPP1116}

SocialMIND ${ }^{\circledR}$ improves attentional performance in schizophrenia spectrum disorder (SDD) patients.

A. Muñoz-Sanjose ${ }^{1 \star}$, R. Mediavilla ${ }^{2}$, A. Pinto García ${ }^{3}$, M.P. VidalVillegas $^{4}$, Í.I. Louzao ${ }^{5}$, P. Aguirre ${ }^{5}$, E.M. Román Mazuecos ${ }^{2}$, P. Sánchez ${ }^{6}$, J. Pastor Haro ${ }^{7}$ and B. Rodríguez-Vega ${ }^{2}$ 
${ }^{1}$ La Paz University Hospital Institute for Health Research (IdiPAZ), Psychiatry, Clinical Psychology and Mental Health, Madrid, Spain; ${ }^{2} \mathrm{La}$ Paz University Hospital Research Health Institute (IdiPAZ), Psychiatry, Clinical Psychology and Mental Health, Madrid, Spain; ${ }^{3}$ Fundación para la Investigación Biomédica del Hospital Universitario La Paz, Mental Health, Madrid, Spain; ${ }^{4} \mathrm{La} \mathrm{Paz}$ University Hospital Biomedical Research Foundation (F.I.B.H.U.L.P.), Psychiatry, Clinical Psychology and Mental Health, MADRID, Spain; ${ }^{5}$ Hospital

Universitario La Paz, Psychiatry, Clinical Psychology and Mental Health, Madrid, Spain; ${ }^{6} \mathrm{La}$ Paz University Hospital, Psychiatry, Clinical Psychology and Mental Health, Madrid, Spain and ${ }^{7}$ Hospital Universitario La Paz, Psiquiatría Y Salud Mental, Madrid, Spain ${ }^{*}$ Corresponding author.

Introduction: Cognitive impairment is usually found in people with Schizophrenia Spectrum Disorder (SDD) (Schaefer et al., 2013). Sustained attention is one of the most characteristic cognitive areas affected and has been largely studied for decades (Hoonakker et al., 2017). Sustained attention is defined as the ability to maintain attention on a given stimulus or task for a span of time (Parasuraman, 1984). Mindfulness is theorized to improve the attentional process. However, the effects of Mindfulness Based Interventions (MBIs) on cognition are still unclear (Lao \& Meadows, 2017).

Objectives: To test if a MBI (SocialMIND ${ }^{\circledR}$ ) improves attentional performance in SDD patients

Methods: A parallel-group, single-blind, randomized, controlled (SocialMIND ${ }^{\circledR}$ versus Psychoeducational Multicomponent Intervention $[\mathrm{PMI}]$ ), clinical trial has been implemented. The participants received 16 weeks group therapy of SocialMIND ${ }^{\circledR}$ or PMI. Sustained attention was measured with the Continuous Performance Test (CPT-IP).

Results: 23 participants were included. A better performance on CPT-IP was found in patients allocated to SocialMIND ${ }^{\circledR}(\mathrm{M}=3.46$, $\mathrm{SD}=0.73)$ compared to $\mathrm{PMI}(\mathrm{M}=2.79, \mathrm{SD}=1.18)$ after sixteen weeks of treatment $(\mathrm{T}=2.89, \mathrm{p}=0.009, \mathrm{~d}=1.19)$. However, this improvement was only found in the 2-digit task.

Conclusions: 3 -digit and 4-digit tasks increase the difficulty of the test involving greater implication of other cognitive processes such as working memory. Taking this into account, it's possible that MBIs improves sustained attention but has no effects on working memory.

Conflict of interest: No

Keywords: Schizophrenia Spectrum Disorder; attention; CPT-IP; Mindfulness

\section{Schizophrenia and other psychotic disorders - Part X}

\section{EPP1121}

\section{Common genes in neurodevelopment and immune- inflammatory pathways impacted in schizophrenia?}

N. Ramoz ${ }^{1 \star}$ and P. Gorwood ${ }^{2}$

${ }^{1}$ INSERM, Institute Of Psychiatry and Neuroscience Of Paris, Paris, France and ${ }^{2}$ Sainte-Anne Hospital, Cmme Department, Paris, France ${ }^{\star}$ Corresponding author.

Introduction: Schizophrenia is a severe disorder affecting $1 \%$ of the general population. Genetic factors have a high impact on this disorder with a heritability estimated between $80-85 \%$.
Furthermore, epidemiological studies showed that infections, autoimmune disorders and immune inflammations are risk factors for the development of psychosis and schizophrenia.

Objectives: The goal of this presentation is to identify the common genes from the neurodevelopment and immune-inflammatory pathways that are potential good biomarkers for the predisposition or the development of psychosis and schizophrenia.

Methods: We took advantages of large genetic studies by genomewide association studies (GWAS) in large cohorts, including investigations on copy number variations (CNVs) and single nucleotide variants, to identify genes of predisposition for schizophrenia and psychosis. Furthermore, integrated network-bases and post-GWAS analyses combined with other omics investigations (like transcriptomics) allowed the identification of enrichments in genes involved in neurodevelopment or in immuneinflammation pathways.

Results: CNVs are well characterized in schizophrenia. They harbor an enrichment of neurodevelopmental genes. Interestingly, some of these CNVs also present an enrichment in microRNAs that specifically target the regulation of expression of neurodevelopmental genes. Furthermore, GWAS and meta-analyses allowed to identify up to 643 risk gene candidates in schizophrenia and, a large set of them represent an enrichment of genes involved in brain development or immunity pathways. Finally, cross-disorder analysis of schizophrenia and 19 immune-mediated diseases identifies shared genetic risk factors.

Conclusions: Clinical diagnosis combined with omics tools will allow to decipher the schizophrenia cases to propose adapted specific treatments for a precision medicine in psychosis and schizophrenia.

\section{Conflict of interest: No}

Keywords: copy number variations; genetic correlation; genomewide association study; microRNA

\section{EPP1126}

\section{Diagnostic stability in the first-episode psychosis}

L. Santos Silva ${ }^{1 \star}$, M. Duarte ${ }^{1}$, R. Araújo ${ }^{1}$, M. Barbosa ${ }^{1}$, J. Miranda $^{1}$ and C. Almeida ${ }^{2}$

${ }^{1}$ Centro Hospitalar de Leiria, Serviço Psiquiatria E Saúde Mental, Leiria, Portugal and ${ }^{2}$ Centro Hospitalar de Leiria, Psychiatry, Leiria, Portugal

${ }^{\star}$ Corresponding author.

Introduction: Early intervention programs in psychosis have demonstrated efficacy in reduction the duration of untreated psychosis, relapse prevention, socio-professional integration and prognosis improvement. Establishing the right diagnosis should be a priority when considering a first-episode psychosis (FEP), as different diagnoses may require different therapeutic approaches. However, this diagnosis is often not possible in the first instance or may change over time.

Objectives: Our study aimed to confirm the diagnostic stability of FEP disorders and to determine whether certain socio-demographic or clinical parameters could influence it.

Methods: We analyzed the clinical date of all 86 patients ever followed at the specific consultation for FEP since its beginning in 2015, with a special focus on initial and current diagnosis.

Results: Among the patients analyzed, most were male and at the time of the first psychotic episode the most common diagnosis was 
Unspecified Nonorganic Psychosis (F29). In more than one third of the cases there was a change in diagnosis, mainly to schizophrenia or substance induzed psychosis. Some patients were excluded since they abandoned their follow-up appointments.

Conclusions: It's important to initially keep an unspecified diagnostic, with further progressive evaluation allowing a more accurate diagnostic, since the initial diagnostic specification is often found to be incorrect, with adverse consequences for the patient. It would be useful to compare the results with a sample of patients under "as usual" treatment.

Conflict of interest: No

Keywords: First-Episode; psychosis; diagnosis

\section{Schizophrenia and other psychotic disorders - Part XII}

\section{EPP1142}

\section{Severe catatonia following quetiapine abrupt withdrawal}

\author{
R. Valido*, P. Frias, F. Caldas and P. Ferreira \\ Hospital de Magalhães Lemos, Psychiatry, Porto, Portugal \\ ${ }^{*}$ Corresponding author.
}

Introduction: Catatonia is a complex psychomotor syndrome and it is recognized that the discontinuation of psychiatric medications can precipitate it.

Objectives: To our knowledge, there are no reports associating quetiapine discontinuation with catatonia. We aim to report a clinical case depicting the unexpected development of severe Catatonia following abrupt discontinuation of quetiapine.

Methods: Clinical case report and a brief review of the literature about withdrawal catatonia.

Results: Quetiapine is mentioned as a possible therapeutic strategy for managing catatonic stupor. The majority of the cases of Catatonia occurred upon benzodiazepines or clozapine discontinuation. None was described occurring with quetiapine. We describe the case of a 66 years old caucasian female admitted at the inpatient unit with a diagnosis of Psychotic Depression, that developed a sudden and unexpected Catatonic state characterized by stupor, negativism, mutism, rigidity, waxy flexibility, and autonomic abnormality 3 days after the abrupt discontinuation of $600 \mathrm{mg}$ of quetiapine because of the rapidly worsening of edema of both legs, presumably caused by the drug. It was excluded neurologic, metabolic or infectious causes. The patient required a nasogastric tube (for nutritional support and medication) and urinary catheterization (urinary retention). Improvements were slow but steady with the introduction of Clonazepam at high doses and Clozapine.

Conclusions: Withdrawal catatonia it's an uncommon yet threatening condition that may arise after a few days of the abrupt interruption of certain medications. However, the etiology of the syndrome is still unknown and we must be aware that additional drugs may precipitate catatonia.
Conflict of interest: No

Keywords: Catatonia; Withdrawal catatonia; Psychotic

Depression; Quetiapine

Sexual medicine and mental health

\section{EPP1155}

\section{Managing antidepressant-associated sexual dysfunction}

D. Esteves-Sousa $^{1 \star}$, N. Moura ${ }^{2}$, M. Albuquerque ${ }^{1}$, J. FacuchoOliveira $^{3}$, P. Espada-Santos ${ }^{1}$, L. Mendonça ${ }^{1}$ and J. Tropa ${ }^{1}$

${ }^{1}$ Hospital de Cascais Dr. José de Almeida, Departamento De Psiquiatria E Saúde Mental, Alcabideche, Portugal; ${ }^{2}$ Centro Hospitalar Lisboa Ocidental, Department of Psychiatry, Lisboa, Portugal and ${ }^{3}$ Hospital de Cascais, Department of Psychiatry, Cascais, Portugal

${ }^{\star}$ Corresponding author.

Introduction: Sexual dysfunction (SD) is a common side effect of antidepressants and can have significant impact on the person's quality of life, relationships, mental health, and recovery. Both depression and its treatments are associated with SD in men and women. The main stages of sexual activity that can be affected are libido, arousal/lubrification, erectile dysfunction (ED) and orgasmic disorders and are differently affected by depression or antidepressants. The rates of SD vary between all antidepressants and with individual susceptibilities. SD is frequently cited as a reason for nonadherence or discontinuation of treatment for depression.

Objectives: Our aim is to perform a review of the literature on the several approaches possible to manage antidepressant sexual dysfunction.

Methods: Our review focuses on articles published within the last 20 years on SD, antidepressants and depression, with particular emphasis on the possible approaches to minimize this side effects. Results: The literature suggests a step-by-step approach: The first being a careful selection of the antidepressant drug (agomelatine, bupropion, trazodone, mirtazapine, vortioxetine and moclobemide); followed by a panel of general interventions (delaying drug intake, omitting doses, waiting for spontaneous remission, or drugswitching); or by an adjunctive intervention (bupropion, buspirone, mirtazapine, trazodone, aripiprazole, PDE-5 inhibitors, testosterone gel, ginkgo biloba, cyproheptadine, loratadine, yohimbine, ropinirole, or amantadine).

Conclusions: Antidepressant sexual dysfunction seems to be dosedependent and normally reversible with discontinuation. Screening for SD and taking specific strategies can minimize or correct this problem. No single approach can be considered 'ideal' so individual assessment on a case-by-case basis is recommended.

\section{Conflict of interest: No}

Keywords: sexual dysfunction; Antidepressants; Depressive disorder; side-effects 
Suicidology and suicide prevention - Part III

\section{EPP1200}

\section{Oedipism - from sophocles's tragedy to present days}

R. Gasparinho*, N. Fernandes, M. Martins, N. Santos, A. Alho, L. Ferreira and A. Ribeirinho

Hospital Distrital de Santarém, Psychiatry and Mental Health, Santarém, Portugal

${ }^{\star}$ Corresponding author.

Introduction: Sophocles' epic Greek mythological tragedy, Oedipus Rex, tells the story of Oedipus whom, after fulfilling the prophecy of killing is father and incestuously marrying his mother, gouged his own eyes. The Bible portraits Oedipism and in myths, legends and cultural tales the concept has also been described. Selfenucleation, auto-enucleation or Oedipism is a major form of selfharm, seldomly reported in literature.

Objectives: Presentation and in-depth discussion of the concept of Oedipism.

Methods: Pubmed search with key terms: "Oedipism"; "Selfenucleation"; "Auto-enucleation"; "Self-harm". Pubmed search with MeSH terms: "Eye Enucleation" AND "Self-Injurious Behavior". Search was restricted to articles written in English, without temporal limitations.

Results: Historically, Oedipism cases were mostly depicted as psycho-sexual or religious conflicts. In psychoanalitic theory, Freud's Oedipal Complex suggests that self-enucleation might be the result of repressed impulses, self-punishment and inward aggression. However, incestuous acts and sexual guilt represent only a minority of the existing reports of Oedipism. It has been associated with severe psychotic disorders, substance-related disorders, severe obsessive-compulsive disorder, mood disorders with psychotic features, severe personality disorders, factitious disorders and incarceration. Remarkably it has also been associated with organic illnesses such as neurosyphilis.

Conclusions: Oedipism is a psychiatric and ophthalmologic emergency. The attempt, act or will to perform self-enucleation translates severe psychopathology yet to be fully understood. In it lies a possible truly devastating physical consequence of severe mental disorders. It is a real challenge to clinicians worldwide. Oedipism demands a tailored approach and remains a rare and serious form of self-harm.

\section{Conflict of interest: No}

Keywords: self-injurious behavior; Oedipism; Eye Enucleation; Self-enucleation

\section{EPP1210}

Autolytic ideation among medical residents in spain: a cross-sectional study

D. Hernández-Calle ${ }^{1 \star}$, A. Cano Arenas ${ }^{1}$, D. Carracedo Sanchidrián ${ }^{1}$ and M.F. Bravo-Ortiz ${ }^{2}$

${ }^{1}$ Hospital Universitario La Paz, Psychiatry, Madrid, Spain and ${ }^{2} \mathrm{La} \mathrm{Paz}$ University Hospital Research Health Institute (IdiPAZ), Psychiatry, Clinical Psychology and Mental Health, Madrid, Spain

${ }^{\star}$ Corresponding author.

Introduction: Several studies have shown an increase in suicidal ideation among physicians and medical students, but there is a lack of information concerning medical residents. This population has high prevalence of depression, anxiety disorders, burnout, bullying and face a tough work-life balance well known risk factors for suicidal thoughts.

Objectives: To study the prevalence and characteristics of suicidal thoughts of medical residents in Spain.

Methods: We conducted a cross-sectional online survey directed to all medical residents in Spain. We analysed the proportion of passive and active suicidal ideation and its association with relevant clinical and sociodemographic variables.

Results: From a total of 653 medical residents, 13.69\% (CI 95\% $11.05-16.34 \%)$ reported passive suicidal ideation, from which $22.47 \%$ were rated as strong. Active suicidal ideation prevalence was $3.52 \%$ (CI 95\% 2.11-4.94), 13.04\% of high intensity. Passive suicidal thoughts were higher in the $3^{\text {rd }}$ year and active in $4^{\text {th }}$, being the $2^{\text {nd }}$ the one with lowest prevalence in both. Between the different specialities groups the rates of active suicidal ideation was estimated to be Central Services: 6.45\% (0.33-12.57\%); Clinical Specialities $4.03 \%$ (2.10-5.96\%); Psychiatrist 2.12\% (0-5.04\%) and Surgical $1.00 \%(0-2.95 \%)$. the proportion of residents with suicidal ideation was attended to mental health consultation was $78.26 \%$.

Conclusions: The prevalence of passive an active suicidal ideation among medical residents in Spain is $13.69 \%$ (CI 95\% 11.05 $16.34 \%$ ) and $3.52 \%$ (CI 95\% 2.11-4.94\%) respectively. The highest risk of active suicidal ideation was founded in last year of the residency program. Central Services specialities had the highest rates.

\section{Conflict of interest: No}

Keywords: Suicidal ideation; Medical Residents

\section{Women, gender and mental health - Part II}

\section{EPP1263}

Women, loneliness, false stigmas and management on frequent attenders of primary care system

L. Giménez-Llort ${ }^{1 \star}$, M. Vidal-Llaudes ${ }^{2}$, A. Odriozola-García ${ }^{2}$ and F. Nunes ${ }^{2}$

${ }^{1}$ Universitat Autònoma de Barcelona, Institute Of Neuroscience \& Department of Psychiatry and Forensic Medicine, Barcelona, Spain and ${ }^{2}$ Universitat Autònoma de Barcelona, Department of Psychiatry and Forensic Medicine, Barcelona, Spain ${ }^{*}$ Corresponding author.

Introduction: There is a trend to define a hyper-frequent patient like the one who, for its age group and sex, has a greater frequency of attending primary care services in a year. No characteristics associated with this patient are defined but the phenomenon it is commonly associated to pluripathology, somatization, depression, anxiety, pessimism and other mental disorders and personality traits. Hyper-attendance seems highly influenced by age (progressive increase) and sex (women more than men). However, the label of frequent users is more a problem than a solution, since it carries a series of prejudices. This stigma points at the elderly population, mostly women, as those 'collapsing' waiting rooms. Although it is known that the phenomenon of frequent attendance has multiple causes and associated factors, the patient is seen as the only responsible. 
Objectives: To perform a survey exploring the perception and clinical practice of physicians and nurses of primary care health centres regarding this issue.

Methods: Retrospective cross-sectional study carried out during three years, in Catalonia, País Valencià and Bask Country.

Results: The characteristics of the frequent attenders, their relationship with the concept of health including well-being, and the professional management of these patients unveiled false stigmas (mostly regarding old women). Results pointed at a current model with poor management of bio-psycho-social dimensions and the loneliness feeling as an important component in frequent attenders of primary care centres.

Conclusions: The bio-psycho-social complexity needs to be taken into account for a better management and improvement of health care quality of the frequent attenders of primary care.

Conflict of interest: No

Keywords: women; Gender Medicine; Loneliness; primary care

\section{EPP1270}

\section{The role of perfectionism and repetitive negative} thinking in prenatal OC phenomena

D. Mota ${ }^{1 \star}$, A.T. Pereira ${ }^{2}$, S. Xavier ${ }^{2}$, J. Azevedo ${ }^{3}$, M.J. Soares ${ }^{2}$, C. Marques ${ }^{3}$, M. Marques ${ }^{4}$, A. Araújo ${ }^{3}$ and A. Macedo ${ }^{2}$

${ }^{1}$ Centro Hospitalar Universitario de Coimbra, Psiquiatria, Coimbra, Portugal; ${ }^{2}$ University of Coimbra, Faculty Of Medicine, Department of Psychological Medicine, Coimbra, Portugal; ${ }^{3}$ Faculty of Medicine, University of Coimbra, Institute Of Psychological Medicine, Coimbra, Portugal and ${ }^{4}$ Coimbra Hospital and University Centre, Clinical Psychology, Coimbra, Portugal

${ }^{*}$ Corresponding author.

Introduction: Perfectionism and repetitive negative thinking has already been associated to perinatal depression and anxiety, but its role in perinatal obsessive-compulsive/OC phenomena has not yet been investigated.

Objectives: To analyze if repetitive negative thinking/RNT mediates the relationship between perfectionism and OC symptoms severity and interference in pregnancy.

Methods: 284 women (Mean age $=32.20 \pm 5.241$ ) in the second trimester of pregnancy (Mean weeks of gestation=17.86 \pm 4.744 ) filled in the Portuguese validated versions of the Prenatal Obsessive Compulsive Scale/POCS, the Hewitt \& Flett Multidimensional Perfectionism Scale-13 and the Perseverative Thinking Questionnaire-15. Hayes' Process Macro for SPSS was used.

Results: POCS_Severity and POCS_Interference significantly ( $\mathrm{p}>$.05) and moderately correlated with Socially prescribed perfectionism/SPP ( $\mathrm{r}=.258, \mathrm{r}=.238$ ), Self-Oriented Perfectionism/SOP ( $\mathrm{r}=.223$ only with POCS_Severity), Repetitive Thinking/RT $(\mathrm{r}=.450, \mathrm{r}=.493)$ and Cognitive Interference and Unproductivity/ $\mathrm{CIU}(\mathrm{r}=.387, \mathrm{r}=.489)$. A mediation analysis (Hays' Model 4) showed that RT/CIU were partial mediators between SPP and POCS_Severity and full mediators between SPP and POCS_Interference. These models explained $20-30 \%$ of the POCS_Severity/Interference variance. The total, direct and indirect effects of SOP on POCS_Severity were not significant.
Conclusions: Negative perfectionism effect on OC Severity and Interference operates via RNT. Given that OC manifestations often first emerges in the antenatal phase and worsens in the postpartum, RNT, as a modifiable cognitive factor, should be targeted in pregnancy to prevent the suffering of new mothers and babies.

\section{Conflict of interest: No}

Keywords: prenatal; obsessive-compulsive disorder; perfectionism

\section{Intellectual disability}

\section{EPP1293}

Features of complex support for children with a delay in the development of psychogenic genesis in an inclusive practice

M. Bratkova, Y. Afanasyeva ${ }^{*}$ and Y. Sidneva

Russian Federation

${ }^{*}$ Corresponding author.

Introduction: Among children with developmental disorders, there is a group with a delay in mental development (DMD), which are most successfully integrated. At present, a group of children with a delay in psychogenic genesis is considered, which needs medical and pedagogical rehabilitation, due to the presence of neurotic disorders and mental distortion.

Objectives: Objective: the study of the psychophysical characteristics of children with DMD psychogenic origin.

Methods: Materials and methods 120 children with DMD psychogenic genesis (2017 - 2018). Methods: medical and pedagogical, observation, examination, assessment.

Results: Variants of the child's psychophysical development: Option 1. (60\%): children with a predominance of violations in behavior, emotional-volitional sphere. Aggression, inconsistency and impulsiveness of actions, lack of distance with adults and difficulties in complying with accepted norms and rules are noted. Option 2 (40\%): children with the following manifestations: shyness, tearfulness, incredulity, fears, lack of creative imagination and expressed interests. Difficulties with sleep, eating disorders, frequent diseases due to psychosomatics.

Conclusions: Children with a delay in psychogenic genesis in conditions of inclusive practice need comprehensive assistance, taking into account different options for their psychophysical characteristics. Neurotic and neurosis-like disorders, pathological personality development were revealed in the studied children. All children do not have enough knowledge about the environment. Low working capacity, lability of the nervous system, lack of arbitrary regulation, impaired activity, learning difficulties. Violations of behavior and psyche are noted, which requires medicalpsychological-pedagogical rehabilitation.

Disclosure: No significant relationships.

Keywords children with a delay in mental development (DMD); inclusive education, developmental delay of psychogenic origin 


\section{E-Poster Viewing}

\section{Addictive disorders}

\section{EPV0015}

\section{Valproate to the rescue: treating a psychotic episode with polymorphic features \\ R. Facão ${ }^{1 \star}$, M. Corvacho ${ }^{2}$, L. Borges $^{1}$, P. Canelas $^{1}$ and J. Silva ${ }^{1}$ \\ ${ }^{1}$ Centro Hospitalar Universitário do Algarve, Psiquiatria, Portimão, Portugal and ${ }^{2}$ Centro Hospitalar Universitário do Algarve, Departamento De Psiquiatria E Saúde Mental, Faro, Portugal ${ }^{\star}$ Corresponding author.}

Introduction: Heterogeneity in treatment response to antipsychotics in schizophrenia is well known. There has been some investigation regarding valproate augmentation therapy in these patients, with positive results in symptoms such as hostility, but also in global function. The literature is scarce concerning patients with substance-induced psychosis.

Objectives: To assess the response to valproate augmentation therapy in a patient diagnosed with psychosis, along with a brief review of the literature on this subject.

Methods: A brief review of the literature is made by using the Pubmed platform applying the terms "psychosis" and "valproate". The patient's clinical status is assessed by means of the Brief Psychiatric Symptom Scale (BPRS) at admittance, and by the BPRS and the Clinical Global Improvement scale (CGI) immediately before and one month after implementing valproate therapy.

Results: Here we report the case of a 25 -year-old male with a history of substance-induced psychosis who was admitted in a psychiatric ward with a psychotic episode. Given that the response to treatment with antipsychotics fell short of what was expected and that we found coexisting affective symptoms, on the 38th day we decided to add valproate to his ongoing treatment. The study is still taking place, thus we have yet to assess the patient's clinical status one month after implementing valproate therapy.

Conclusions: We expect to highlight the relevance of a symptomoriented approach to treating patients with polymorphic clinical pictures, as well as the need for further research regarding this subject.

Conflict of interest: No

Keywords: psychosis; Valproate

\section{EPV0034}

Gamma-hydroxybutyric acid (GHB) detoxification: a review.

\section{E. Rodríguez}

Hospital Clínic Barcelona, Institute of Neuroscience, Barcelona, Spain

Introduction: Gamma-hydroxybutyric acid (GHB) is a substance used as a recreational drug with a significant dependence potential. Its consumption has lately increased, the same as cases suffering withdrawal syndrome, which appears rapidly and can become critical. Benzodiazepines are used as first choice agent to treat GHB withdrawal syndrome.

Objectives: To analyse gamma-hydroxybutyric detoxification treatment and alternatives studied since the moment.
Methods: Bibliographic review to analyse gamma-hydroxybutyric acid detoxification: pharmaceutical GHB compared to benzodiazepines.

Results: Due to specificity of gamma-hydroxybutyric acid's action, its withdrawal syndrome's management can be difficult using only benzodiazepines, gabapentin or antipsychotics because withdrawal symptoms can not all be related to a single dominant mechanism or neurological pathway. Analyzing neurobiological mechanisms of GHB dependence, it would make sense to treat its abstinence syndrome with the same substance, expecting it to be more specific and effective. An observational study developed a Detoxification procedure by Titration and Tapering (DeTiTap procedure) with off-label use of pharmaceutical GHB as an alternative treatment option to benzodiazepinas.

Conclusions: 1 . The daily amount of gamma-hydroxybutyric acid use has been rapidly increased (1). 2. Gamma-hydroxybutyric acid withdrawal syndrome can be life threatenting. Its treatment is difficult with a single drug class because of the high complex interaction of neurobiological mecanisms where GHB involves $(2,3)$. 3. Since the moment, only observational studies have been performed to determinate efficacy of GHB to treat its withdrawal syndrome using the Detoxification procedure by Titration and Tapering. Randomized controlled trials are required to establish efficacy and safety (4).

\section{Conflict of interest: No}

Keywords: Gamma-hydroxybutyric acid; Dependence; withdrawal; Detoxification

\section{EPV0035}

Psychopharmacological management of the abstinence and craving stages of alcohol dependence: evidencebased recommendations

\section{P. Sousa Martins ${ }^{\star}$ and M. Bettencourt}

Unidade Local de Saúde do Nordeste, E.P.E., Departamento De Psiquiatria E Saúde Mental, Bragança, Portugal

${ }^{\star}$ Corresponding author.

Introduction: Therapeutic intervention of alcohol abstinence is usually the first step of an intricate treatment process that is followed up by the control of the "irresistible desire to drink"commonly known as craving. In both moments, resorting to the aid of psychopharmacology (a strategy not sufficient, but oftentimes necessary) should be done in a highly careful manner as to obtain the desired results with the least possible side effects.

Objectives: With the current review, we set out to pinpoint which medications have clear and robust evidence for usage in both the abstinence and craving stages of alcohol dependence.

Methods: A systematic review of the current literature, using the words "alcohol”, "dependence", "abstinence”, "craving", "pharmacological" and "evidence" in the PubMed database's search domain. Results: Current literature suggests that benzodiazepines are the best drugs to control the abstinence symptoms that mark the first stage of the alcohol dependence treatment; benzodiazepines with a more diffuse plasmic peak concentration and intermediate half-life should be given preference; in special situations like alcoholic delirium or sub-acute delirium, neuroleptic medication should be the first choice. Craving reduction resorting to psychopharmacological methods hasn't always received robust evidence; acamprosate and naltrexone are the drugs with better evidence for usage; selective serotonin reuptake inhibitors (SSRI), disulfiram or other molecules like nalmefene can be useful in particular clinical situations. 
Conclusions: Psychopharmacology is an invaluable tool for the management of the various stages of alcohol dependence. Its usage should be done with extreme precaution and always bearing in mind what interventions are clearly based on robust scientific evidence.

Conflict of interest: No

Keywords: Alcohol; abstinence; craving; psychopharmacology

\section{EPV0043}

\section{Alcoholic hallucinosis, a case report.}

Y. Dhiver Cantalejo $^{1 \star}$, G. Sánchez Amador ${ }^{2}$ and P. Padilla Romero ${ }^{2}$

${ }^{1}$ Hospital General Nuestra Señora del Prado, Psychiatry, Talavera de la Reina, Toledo, Spain and ${ }^{2}$ Hospital General Nuestra Señora del Prado, Psychiatry, Talavera, Toledo, Spain

${ }^{\star}$ Corresponding author.

Introduction: Alcoholic hallucinosis is an organic psychotic disorder that goes with vivid and persistent hallucinations and usually appears within 48 hours after the interruption or reduction of prolonged and intense alcohol consumption. When it stays beyond six months, maintaining abstinence, it can evolve into an organic dementia or schizophrenia paranoid

Objectives: Presentation of a clinical case

Methods: We analyze the case of a 44 -year-old male who enters due to persistence of auditive hallucinations that he describes as murmurs, insulting and accusatory voices in the third person, delusional interpretations with affective repercussions. In addition to that dull thought and intense asthenia. Dipsomaniac drinker for years of high amounts of alcohol. Total abstinence for 15 days.

Results: during admission, doses of up to $20 \mathrm{mg}$ of olanzapine were prescribed, which were reduced to $5 \mathrm{mg}$ due to excessive drowsiness, lack of concentration and attention and bradyness; with adequate response. Do not be objective after decreased recurrence of sensory symptoms. You do not have alcohol withdrawal symptoms during your stay. Analytics were performed that included blood count, biochemistry, coagulation, lipid, ferric and vitamin profile, resulting normal and negative toxins. Brain MRI was normal.

Conclusions: It is important in the cases of patients with alcohol consumption and hallucinations clinic, to rule out a delirium tremens, since this could lead to the death of the patient if not treated. In certain cases it will also be necessary to make a differential diagnosis with schizophrenia, schizophreniform disorder and brief reactive psychosis.

Conflict of interest: No

Keywords: Alcoholic hallucinosis; delirium tremens; schizophrenia; Dipsomaniac drinker

\section{EPV0044}

\section{Amphetamine psychosis, a case report}

Y. Dhiver Cantalejo ${ }^{1 \star}$, P. Padilla Romero ${ }^{2}$, G. Sánchez Amador ${ }^{2}$, E. Mancha Heredero ${ }^{3}$ and M.T. Pérez Castellano ${ }^{2}$

${ }^{1}$ Hospital General Nuestra Señora del Prado, Psychiatry, Talavera de la Reina, Toledo, Spain; ${ }^{2}$ Hospital General NuestraSeñora del Prado, Psychiatry, Talavera, Toledo, Spain and ${ }^{3}$ Hospital ClínicoUniversitario, Psiquiatria, Valladolid, Spain ${ }^{\star}$ Corresponding author.

Introduction: During chronic consumption of psychostimulants, many consumers may experience a psychotic disorder. It is a group of psychotic phenomena that occur during the consumption of a psychoactive substance or immediately after it, but are not explained by acute poisoning alone and are not part of the withdrawal syndrome. Objectives: Presentation of a clinical case

Methods: Analyzing the caso of a 50-year-old male with a history of polyconsumption (heroin, cocaine, cannabis and alcohol) and diagnosis of adult $\mathrm{ADH}$, who is admitted for an episode of agitation in the context of chronic consumption of prescription amphetamine derivatives used for the treatment of childhood ADH. Delusional interpretations of the environment are collected with ideation of persecution and vigilance, irritability, distrust and finally aggressive behavior.

Results: Dosage of methylphenidate was reduced gradually with adequate response and aripiprazole was introduced up to a dose of $30 \mathrm{mg} / 24 \mathrm{~h}$. The psychotic clinic completely yielded with complete criticism of the experience. Interventions are carried out to promote awareness of symptoms, but achieving it partially. Analytics were performed that included hemogram, biochemistry, coagulation, lipid, ferric and vitamin profile, resulting normal. Brain MRI was normal. Conclusions: It is important to make a proper differential diagnosis between acute episodes of substance intoxication / withdrawal and psychotic episodes. Psychosis caused by amphetamines, cocaine, or phencyclidine may persist for several weeks. Because some young people with schizophrenia in the prodromal or initial phase consume substances that can induce psychosis, it is essential to see the evolution.

\section{Conflict of interest: No}

Keywords: ADH; psychosis; Amphetamine; psychostimulants

\section{EPV0045}

\section{Psychopharmacologic automedication in medical} residents in spain: a cross-sectional study.

\section{Hernández-Calle ${ }^{1 *}$, D. Carracedo Sanchidrián ${ }^{1}$, A. Cano Arenas $^{1}$ and M.F. Bravo-Ortiz ${ }^{2}$ \\ ${ }^{1}$ Hospital Universitario La Paz, Psychiatry, Madrid, Spain and ${ }^{2} \mathrm{La} \mathrm{Paz}$ University Hospital Research Health Institute (IdiPAZ), Psychiatry, Clinical Psychology And Mental Health, Madrid, Spain ${ }^{*}$ Corresponding author.}

Introduction: Medical residents suffer from high rates of depression and anxiety disorders, as well insomnia due to long working shifts. This could increase the risk of using psychoactive medication without being assessed by a specialist (auto medication), which can lead to substance-related disorders due to its dependence potential. Objectives: To estimate the prevalence of automedication in Medical residents in Spain.

Methods: We conducted a cross-sectional online survey directed to all medical residents in Spain and analysed the proportion of automedication and clinically significant related variables. A multivariate analysis was performed to study potential risk factors.

Results: From a total of 656 Medical residents, 19.20\% (95\%CI 16.19-22.22\%) reported to have medicated themselves, mostly with Benzodiazepines (15.87\%). Psychiatrist had the highest estimated prevalence $(36.84 \%$; $27.14-46.54 \%)$ with an OR of 3.09 (1.86-5.15) compared their fellow residents. Passive suicidal ideation was founded to be most important risk factor (OR 3.68; 1.98-6.82).

Conclusions: The estimated prevalence of automedication with psychoactive drugs in Medical residents in Spain was $19.20 \%$ (95\%CI 16.19-22.22\%). More than one third of psychiatric resident reported to medicate themselves. Being a psychiatric resident (OR $3.09 ; 1.86-5.15)$ and suffering from passive suicidal ideation (OR 3.68; 1.98-6.82 were founded to be the highest risk factors. 
Conflict of interest: No

Keywords: Medical Residents; Automedication

\section{Anxiety disorders and somatoform disorders}

\section{EPV0090}

\section{Takotsubo myocardiophaty and anxiety: a case report}

L. Carpio Garcia*, C. Martín Villarroel, J. Dominguez Cutanda, G. Belmonte Garcia and M. Fernandez Torija Daza

HOSPITAL PROVINCIAL DE LA MISERICORDIA, PsiquiatrÍa (consultas Externas), TOLEDO, Spain

${ }^{\star}$ Corresponding author.

Introduction: Tako-Tsubo Syndrome (TTS) is a reversible clinical entity that simulates acute myocardial infarction without the evidence of obstructive-coronary-artery-disease. Takotsubo myocardiopathy is closely related to intense emotional events, and usually occurs in postmenopausal women. Whether the vulnerable substrate for TTS is the history of a mood or anxiety disorder in itself, due to the chronic distress, or the antidepressant drugs, both psychiatric illness and treatment remains unclear.

Objectives: To evaluate the association between anxiety disorders and TTS.

Methods: We analyse through two cases how anxiety and mood disturbance may be linked to the evolution of TTS. A bibliographic search was performed from different databases (Pubmed, Ovid, ScienceDirect) about this topic. We present two patients that have been referred in the last year to our unit due to this myocardiopathy. One of them was a 48-year-old-woman, diagnosed with anxiety disorder and suffered two episodes of takotsubo reactive to intense anxiety crises. The other woman was diagnosed with MixedAdaptive Disorder and suffered this cardiac event on one occasion. The level of anxiety was very high and was poorly controlled in both patients.

Results: Both cases were managed through medication based on antidepressants, anxiolytics and psychotherapy. Both women are still in treatment showing a significant improvement in the level of anxiety, and have not presented recurrence of new cardiac events. Conclusions: This cardiac pathology can be serious if it is not treated in an appropriate manner. The treatment of anxiety disorders through medication and psychotherapy techniques that help to improve skills that allow its management, is very important.

Conflict of interest: No

Keywords: Anxiety; stress; Tako-Tsubo cardiomyopathy

\section{EPV0091}

Resistant anxiety and affective instability as a marker of abuses in the past: a case report

L. Carpio Garcia*, C. Martín Villarroel, J. Dominguez Cutanda, G. Belmonte Garcia and M. Fernandez Torija Daza

HOSPITAL PROVINCIAL DE LA MISERICORDIA, PsiquiatrÍa (consultas Externas), TOLEDO, Spain

${ }^{\star}$ Corresponding author.

Introduction: In our clinical practice we find patients with anxiety and mood instability that are resistant to different strategies of medical treatment and psychotherapy. It is relevant that many of them have recognized a history of abuses in their past that never before was detected neither solved. It is known that Affectiveinstability (AI), childhood trauma, and mental illness are linked, but evidence in mood disorders is limited.

Objectives: The aim of this paper is to document resistant anxiety and AI cases with history of adverse childhood experiences, in order to determine refractory mood disorders as a marker of history of abuses in the past.

Methods: A bibliographic search was performed from different databases (Pubmed,Ovid,ScienceDirect)about this topic, from a case series report, showing throw aspects related to mood dysregulation in adults with childhood maltreatment.

Results: A remarkable percentage of the evaluated patients diagnosed with refractory anxiety or AI, had a history of childhood trauma, what might be an adaptive response to stressors and attenuate affective instability.

Conclusions: Childhood-maltreatment has been shown in metaanalyses to be a major risk factor for mood and anxiety-disorders, also there is a strong link between childhood traumatic events and AI levels. For that reason, if we identify a refractive course of anxietymood disorder as a marker of vulnerability of history of trauma in the childhood, that could potentially optimize service delivery and therapy outcomes among that profile of patients. More studies are required to achieve an adequate evaluation, allowing a different inicial boarding that could modify the course,thanks to its early detection.

Conflict of interest: No

Keywords: Affective instability; trauma; Anxiety; Abuse

\section{EPV0093}

\section{Specific phobia, a case report}

Y. Dhiver Cantalejo ${ }^{1 \star}$, G. Sánchez Amador ${ }^{2}$ and P. Padilla Romero ${ }^{2}$

${ }^{1}$ Hospital General Nuestra Señora del Prado, Psychiatry, Talavera de la Reina, Toledo, Spain and ${ }^{2}$ Hospital General Nuestra Señora del Prado, Psychiatry, Talavera, Toledo, Spain

${ }^{\star}$ Corresponding author.

Introduction: A specific phobia consists of an intense and persistent pathological fear or anxiety for a specific object, animal, situation or activity that is not proportional to the real risk, there being an oversize of it.

Objectives: Presentation of a clinical case

Methods: Analyzing the case of a 49-year-old woman who claims to never have gone to the dentist because of the fear and anxiety that it causes, a situation that has deteriorated her teeth over time, presenting root remains that advise her to be extracted. From the knowledge of the procedure, she presents a state of generalized anxietyand avoidance behaviors such as looking in the mirrors and that people see it as a shame ... she says she fears local anesthesia since "I am not going to overcome it" while demanding a solution. To the psychopathological exploration, resonant mood, syntonic contact, fluid, structured and coherent discourse, cognitive and perceptual distortions congruent with the phobic content are objective with avoidance behaviors without a delusional component or sensory perception alterations. Insomnia of conciliation

Results: The patient has a specific phobia to go to the dentist with marked impact and interference in their daily functioning. A possible delusional character is ruled out in it. It is advisable to treat your dental problem with general anesthesia to be able to work later in therapy with a cognitive-behavioral approach. Pharmacological treatment with paroxetine $20 \mathrm{mg} / 24 \mathrm{~h}$ and diazepam $10 \mathrm{mg} / 12 \mathrm{~h}$ is prescribed.

Conclusions: The first-line treatment for specific phobia is cognitive behavioral therapy 
Conflict of interest: No

Keywords: cognitive behavioral; phobia; Anxiety; SSRI

\section{EPV0101}

\section{Psychological factors predict electrodiagnostic studies results}

M. Papadopoulou ${ }^{1}$, I. Michopoulos ${ }^{2 \star}$, G. Tsivgoulis ${ }^{3}$, L. Palaiodimou ${ }^{3}$, M. Bregianni ${ }^{3}$ and K. Voumvourakis ${ }^{3}$

${ }^{1}$ University of West Attica, Department of Physiotherapy, Athens, Greece; ${ }^{2}$ National and Kapodistrian University of Athens, 2nd Department of Psychiatry, Attikon General Hospital, Athens, Greece and ${ }^{3}$ National and Kapodistrian University of Athens, 2nd Department of Neurology, Attikon Hospital, Athens, Greece

${ }^{*}$ Corresponding author.

Introduction: There is a great amount of normal results in requested medical exams. Electrodiagnostic studies (EDX) investigate peripheral nervous system and produce normal results in a high proportion (35\% to 69\%). Several studies have attributed this to the inappropriateness of the referral and even related it to the specialty of the referral physician. Other studies do not confirm the above notion.

Objectives: The aim of our study was to investigate psychometric characteristics of the referred population and their impact on EDX result.

Methods: We prospectively enrolled 100 patients that were referred for EDX study of the upper limbs and recorded their demographic, biochemical and psychometric characteristics (applying HADS and WHODAS).

Results: Normal results were elicited in 56 patients. From all factors examined, only age and numbness in right hand were associated to EDX result. Although, women were the majority (78\%), there was no statistical difference of abnormal EDX studies between the two sexes. Moreover, men and women did not differ in any other factor, except HADS-D and WHODAS.

Conclusions: Women more often than men, most probably due to psychological reasons, seek medical attention for symptoms that prove to be innocent and might reflect patients' vague agony regarding their health.

Conflict of interest: No

Keywords: Electrodiagnostic studies; Psychological factors; HADS; WHODAS

\section{EPV0112}

\section{Revisiting psychogenic non-epileptic seizures: a literature review}

\author{
L. Santos Silva ${ }^{\star}$ C. Almeida, M. Barbosa, J. Miranda and M. Duarte \\ Centro Hospitalar de Leiria, Serviço Psiquiatria E Saúde Mental, Leiria, \\ Portugal \\ ${ }^{\star}$ Corresponding author.
}

Introduction: Initially identified by Jean-Martin Charcot, nonepileptic psychogenic seizures (PNES) are today described as paroxysmal episodes that resemble and are often diagnosed as epileptic seizures, however, having a psychological rather than neurological origin.

Objectives: The authors propose to carry out a review of the available literature regarding these relatively common phenomena, reflecting on the epidemiology, etiology and frequent comorbidities, diagnosis, therapeutic interventions and expected prognosis. Methods: Medline database search through the Pubmed search engine with the terms "Psychogenic non-epileptic seizures", and the articles that best corresponded to the authors' objectives were selected.

Results: According to the existing literature, 5-10\% of outpatient epileptics and $20-40 \%$ of hospitalized epileptics have PNES. Onset of seizures occurs most often in young adults, mostly affecting women. Common psychiatric comorbidities include depressive disorders, PTSD, other dissociative or somatoform disorders, and personality disorders (especially borderline). Many patients have a history of traumatic events (eg sexual abuse or physical violence). VEEG is the gold standard for diagnosis and post-diagnosis therapeutic interventions include discontinuation of antiepileptic drugs, referral to mental health care and cognitive behavioral therapy as an important part of treatment.

Conclusions: Patients with PNES are often misdiagnosed and treated as having epilepsy over several years, with all the consequences this entails, including the use of antiepileptic drugs and various unnecessary invasive interventions, as well as the associated costs. It is therefore important for the clinician to know the signs indicating the presence of PNES in order to ensure a correct diagnosis in a timely manner.

Conflict of interest: No

Keywords: psychogenic seizures; non-epileptic seizures; seizures

\section{EPV0116}

Psychosomatic symptoms and affective disorders: an exploratory data analysis.
R. Fernández ${ }^{1}$, N. López ${ }^{2}$, N. Mesa ${ }^{1}$ and L. Caballero Martínez ${ }^{1}$
${ }^{1}$ HM CINAC, Servicio De Psiquiatría Y Psicología Clínica Hm
Hospitales, Móstoles, Spain and ${ }^{2}$ Universidad San Pablo CEU, Facultad De Medicina, Móstoles, Spain
${ }^{\star}$ Corresponding author.

Introduction: Clinical and epidemiological studies have found that the co-occurrence of some somatoform symptoms with anxiety and depressive disorders is common (Lieb R, 2007). These results are highly relevant in terms of health policies.

Objectives: We intend to carry out an exploratory data analysis of all the patients who attend a Psychiatry consultation due to probable psychosomatic symptoms.

Methods: We have conducted a search of all patients who attend a psychiatric consultation and have classified them according to referral for psychosomatic symptoms or not. We subsequently carried out a descriptive analysis in which we included: age, time in days until referral, diagnostic group and type of symptomatology.

Results: The median age of patients referred for psychosomatic symptoms is 43 years (standard deviation of 14.49). The average number of days it took to refer the patient to Psychiatry was 124.56 , with a range between 1 and 912 days. The majority of patients were referred by Internal Medicine (42.1\%) and Neurology (47.4\%). $21.1 \%$ of referred patients had a psychiatric history of anxiety disorder, $15.8 \%$ had a history of depressive disorder. The most frequent diagnoses made in this population were depressive disorders (36.9\%) and anxiety disorders (63.2\%), followed by personality disorder (10.5\%). Interestingly, the diagnosis of somatomorphic disorder was only codified in $10.5 \%$ of patients. 


\begin{tabular}{lll}
\hline & Frequenty & Percentage \\
\hline Primary Care & 1 & 5.3 \\
\hline Cardiology & 1 & 5.3 \\
\hline Internal Medicine & 8 & 42.1 \\
\hline Neurology & 9 & 47.4 \\
\hline Total & 19 & 100 \\
\hline
\end{tabular}

Conclusions: These results underline the necessity to implement appropriate strategies to improve early detection of patients (Piontek K., 2018).

Conflict of interest: No

Keywords: anxiety disorders; depressive disorders; somatoform disorders; exploratory data analysis

\section{Bipolar disorders}

\section{EPV0121}

\section{Steroid-induced mania. Clinical features and treatment. About a case.}

C. Carrajo Garcia ${ }^{\star}$, M. Rodriguez De Lorenzo, E. Alonso Sánchez and C. Loeck De Lapuerta

HOSPITAL UNIVERSITARIO RAMON Y CAJAL, Psychiatry, MADRID, Spain

${ }^{\star}$ Corresponding author.

Introduction: A 68-year-old woman, who goes to the Mental Health Center, accompanied by her daughter, claiming to ignore the reason for consultation. Presents a speech of megalomaniac dyes on his intellectual capacity, a noble title and an alleged power to cure cancer. Verbalize delusional ideas of damage with a high level of structuring, around his sister-in-law, the porter and financial entities. He has increased his expenses in recent times and has in mind to initiate several projects, such as a charitable foundation for children. His relatives comment that he began with behavioral alterations of this type, irritability and episodes of verbal heteroagressivity compared to minimum confrontations two months before coinciding with the end of a treatment with chemotherapy ( 3 cycles of ifosfamide) and corticosteroids at high doses due to a uterine carcinosarcoma currently in remission.

Objectives: The aim of this case is to show the neuropsychiatric effects of steroid drug and its treatment.

Methods: The superior psychic functions are preserved and in the imaging tests there are no significant findings that explain the clinic we expose.

Results: The management was ambulatory with olanzapine (10 mg / day), thanks to good family containment and the patient presented a favorable evolution, improving behavioral alterations and mitigating the delusional theme.

Conclusions: Steroid-induced mania is a dose dependent reaction that has a remission close to $90 \%$ after withdrawing the drug. Olanzapine has been placed as the gold standard in cases in which symptomatology persists despite withdrawal or when high functional impact is presented.

\section{Conflict of interest: No}

Keywords: olanzapine; corticosteroids; mania; oncological patient

\section{EPV0135}

Is functional impairment stable in patients with bipolar disorder and schizophrenia over time?

A. López-Villarreal ${ }^{1 \star}$, E. Jiménez-López ${ }^{2}$, E.M. Sánchez-Morla ${ }^{3}$, A. I. Aparicio ${ }^{4}$, R. Rodriguez-Jimenez ${ }^{3}$, P. Vidal Pérez ${ }^{5}$ and J.L. Santos ${ }^{4}$

${ }^{1}$ Hospital Virgen de La Luz, Departament Psychiatry, Cuenca, Spain;

${ }^{2}$ Universidad de Castilla La Mancha, Health And Social Research Center Of Cuenca, Cuenca, Spain; ${ }^{3}$ Hospital Universitario 12 de Octubre, Madrid, Spain, Department of Psychiatry, Madrid, Spain;

${ }^{4}$ Hospital Virgen de la Luz, Department of Psychiatry, Cuenca, Spain and ${ }^{5}$ Consorci Corporació Sanitària Parc Taulí de Sabadell, Psychiatry, Barcelona, Spain

${ }^{\star}$ Corresponding author.

Introduction: Patients with schizophrenia (SZ) and bipolar disorder (BD) share a wide range of neurocognitive deficits, whose longterm course stills nuclear (Balanza et al., 2009). Some studies have found a progressive impairment of cognitive function in patients with SZ, whereas others have observed stability, or even an improvement in some cognitive domains (Szoke et al., 2008). Additionally, it has been suggested that neurocognitive deficits of patients with $\mathrm{BD}$ remain stable throughout time.

Objectives: To examine the course of psychosocial functioning in a sample of patients stable with a diagnosis of SZ and euthymic patients with $\mathrm{BD}$, related to healthy controls ( $\mathrm{HC}$ )

Methods: Fifty-five euthymic patients with BD, 76 stabilized patients with SZ and $25 \mathrm{HCs}$ were assessed at the beginning of the study (T1) and after five years (T2). The clinical status was determined using the Spanish version of the Positive and Negative Syndrome Scale (PANSS), the Hamilton Depression Rating Scale (HDRS), and the Young Mania Rating Scale (YMRS). Additionally, the functional outcome was assessed with the Spanish version of the Quality of Life Scale (QLS) and the Global Assessment Functioning Scale (GAF). The statistical analysis was carried out with repeated measures MANOVA. Age, sex and education were used as covariates.

Results: The repeated-measures MANOVA did not show the existence of a significant group $\mathrm{x}$ time interaction for the Clinical and functional variables. There was no effect of time for any functional measure.

Conclusions: The impairment of psychosocial function in patients with $\mathrm{BD}$ and $\mathrm{SZ}$ is stable.

Conflict of interest: No

Keywords: Bipolar disorder; follow up; psychosocial functioning; schizophrenia

\section{EPV0146}

\section{Impulsivity in bipolar disorder. Comorbidity with} attention deficit hyperactivity disorder.

A. Rodríguez-Quiroga ${ }^{1 \star}$, P. $\mathrm{Nava}^{2}$, A.M. Matas Ochoa ${ }^{1}$, R. Martinez De Velasco ${ }^{2}$, C. Banzo ${ }^{2}$ and F. Mora ${ }^{2}$

${ }^{1}$ Hospital Universitario Infanta Leonor, Psychiatry, Madrid, Spain and ${ }^{2}$ Hospital Infanta Leonor, Psychiatry, Madrid, Spain

${ }^{*}$ Corresponding author. 
Introduction: $\mathrm{BD}$ and ADHD are highly prevalent neurodevelopmental disorders, with an early onset age and a chronic course. Comorbidity rates between ADHD and BD vary between 9.5 and $30 \%$. This has been systematically associated with an earlier age at the onset of bipolar disorder and a more chronic and disabling course of the disorder.

Objectives: The aim of the study is to shed light on the comorbidity of $\mathrm{BD}$ and $\mathrm{ADHD}$ in routine clinical practice.

Methods: We describe the case of a 20 -year-old male patient diagnosed with bipolar disorder (BD), attention deficit hyperactivity disorder (ADHD) and tic disorder. Following the initial evaluation, in which both diagnoses were confirmed, he begun with frequent tics and intense mood swings.

Results: The psychopathological examination was characterized by recurring distractions and difficulties in maintaining attention. Slight psychomotor restlessness. Mood swings showing a low mood tendency, without major depressive episode criteria. Impulsiveness. Judgment of reality preserved.

Complementary tests (complete blood and urine analysis, electrocardiogram, as well as imaging test consisting of computerized axial tomography) were within the normal range, except for cannabis positivity.

The differential diagnosis was made between TBP and ADHD. Valproic acid and guanfacine were prescribed, which has been shown to improve the tics and symptoms of ADHD in a specific group of patients.

Conclusions: This particular case made us consider the spectrum of impulsivity, in which many patients with different diagnoses converge. Knowing the high comorbidity rates between $\mathrm{BD}$ and $\mathrm{ADHD}$, as well as the different therapeutic options, is essential, since it is the only way to improve the outcome.

Conflict of interest: No

Keywords: Bipolar disorder; Tics; ADHD; Impulsivity

\section{Bipolar disorders}

\section{EPV0152}

\section{Blood test to diagnose bipolar disorder using rna editing-related biomarkers}

F. Chimienti ${ }^{\star}$, N. Salvetat, P. Dupré, B. Dubuc, C. Cayzac, F. Checa-Robles, V. Patel and D. Weissmann

ALCEDIAG/SYS2Diag, Montpellier, France

${ }^{\star}$ Corresponding author.

Introduction: Bipolar disorder (BD) is comprised of fluctuating episodes of both depression and mania or hypomania. During depression stage bipolar patients are often misdiagnosed. There is no objective laboratory-based test or biological markers to discriminate unipolar from bipolar depression; the average interval between onset and proper diagnosis and treatment is estimated to be 5.8 years. Altered epigenetic mechanisms, including abnormal RNA editing, are involved in neuropsychiatric disorders like schizophrenia, Bipolar Disorder and Major Depressive Disorder. Objectives: We focused on transcriptome-wide RNA editing modifications on Unipolar and BD patients to identify RNA editing variants that could be used as biomarkers to set up a clinically useful diagnostic assay for differential diagnosis of BD.

Methods: We used whole-transcriptome sequencing (RNA-Seq) to identify differential A-to-I editing events in blood samples of
Unipolar $(n=13)$ and BD $(n=14)$ patients and Targeted Next Generation Sequencing to validate those biomarkers in a large cohort of 297 patients (Unipolar, $n=160$ and $B D, n=96$ ).

Results: We identified significant differential editing variants in a number of genes involved in different pathways relevant for mood disorders, e.g. immune system and CNS function. We selected a panel of 8 biomarkers which are simultaneously measured in patients' blood and rise specific signatures in man and women related to unipolar and bipolar depressive conditions. The test presents high performances ( $>80 \%$ specificity and selectivity).

Conclusions: Our findings will contribute to a better understanding of the molecular pathophysiology of $\mathrm{BD}$, and pave the way for the development of a diagnostic assay for BD with clinical application.

Conflict of interest: No

Keywords: diagnostic; RNA editing; Bipolar disorder

\section{Child and adolescent psychiatry}

\section{EPV0188}

\section{Emotional regulation in infants born after a threatening preterm labour: a prospective study.}

L. Campos-Berga $^{1 \star}$, A. Moreno-Giménez ${ }^{2}$, R. Sahuquillo-Leal ${ }^{2}$ and A. García-Blanco

${ }^{1}$ UNIVERSITY AND POLYTECHNIC HOSPITAL LA FE, VALENCIA, Psychiatry And Psychology, VALENCIA, Spain;

${ }^{2}$ University of Valencia, Psychology, VALENCIA, Spain and

${ }^{3}$ University of Valencia// Health Research Institute La Fe, Psychology// Neonatology, VALENCIA, Spain

${ }^{\star}$ Corresponding author.

Introduction: Prematurity is associated with neurodevelopmental disorders such as cognitive impairment, psychomotor delay, autism spectrum disorders (ASD), and attention deficit hyperactivity disorder (ADHD), among others. Both psychomotor and emotional competences are considered early manifestations of neuropsychiatric disorders. On the other hand, maternal stress and obstetric events can also affect infant neurodevelopment and has been linked to emotional disturbances at later stages, regardless of not being premature.

Objectives: A threatened preterm labour (TPL) episode represents a stressful prenatal event and a potential risk factor for neuropsychiatric disorders. The aim of this study was to characterize temperament and emotional regulation of infants whose mothers suffered from a TPL although finally completed a full-term gestation.

Methods: This prospective cohort study recruited mothers who suffer from a TPL $(n=144)$ and conducted a follow-up of their offspring. A control group of mothers without TPL and full-term gestation $(n=63)$ completed the sample. Newborn outcomes were registered and infants' temperament assessment was performed at 4-6 months using The Infant Behaviour Questionnaire-Revised (IBQ-R) short version.

Results: Using ordinal regression models, after including delivery week, multiple pregnancy, in vitro fertilization, and infant sex as covariates, TPL infants showed emotional dysregulation (higher negative affect, lower positive affect, and worse emotional regulation) compared to the control group.

Conclusions: TPL, regardless of the delivery week, may represent a risk factor for neurodevelopmental disturbances in the offspring, 
with a detrimental impact on emotional competences, which have been considered as early manifestations of later neuropsychiatric disorders.

\section{Conflict of interest: No}

Keywords: threatening preterm labor (TPL); prenatal stress; neurodevelopment; emotional regulation

\section{EPV0191}

\section{The boy who needed more than pills}

C. Carrajo Garcia ${ }^{1}$, C. Loeck De Lapuerta ${ }^{1}$, M. Rodriguez De Lorenzo $^{1}$, E. Alonso Sánchez ${ }^{1}$ and P. Del Sol Calderón ${ }^{2}$

${ }^{1}$ HOSPITAL UNIVERSITARIO RAMON Y CAJAL, Psychiatry, MADRID, Spain and ${ }^{2}$ HOSPITAL UNIVERSITARIO PUERTA DE

HIERRO MAJADAHONDA, Psychiatry, MADRID, Spain

${ }^{\star}$ Corresponding author.

Introduction: 16-year-old male in follow-up with Neurology and Psychiatry from the age of 6 , with poor tolerance to different pharmacological lines. He has received the diagnoses of Autism Spectrum Disorder, Attention Deficit Hyperactivity Disorder and Tics Disorder. He has not performed psychotherapeutic treatment prior.

Objectives: The main objective is to highlight the importance of psychotherapy in neurodevelopmental disorders.

Methods: He was adopted alone by his mother at age three. In the initial joint interview, an authoritarian parental style is observed, with deregulation between affection / communication and control / demand. Subsequent individual evaluations reveal difficulties in communication and social interaction, the need for rigid routines and poor cognitive flexibility, as well as depressive symptoms derived from poor self-concept. There is a disagreement between the demand raised by the mother (pharmacological adjustment) and the son, who focuses on interpersonal relationship difficulties (difficulty inferring mental states from others, impulsivity and inadequacy in response).

Results: In the therapeutic setting (outpatient intervention), in addition to the pharmacological adjustment, the following objectives were raised: -To promote autonomy and interpersonal functioning on the one hand and effective and bidirectional communication on the other hand, through family and interpersonal therapy. -Increase cognitive flexibility and reduce the level of anxiety in situations beyond control, through cognitive-behavioral interventions. -Training in identification and regulation of emotions, assertive communication and social cognition.

Conclusions: Although the psychotherapeutic intervention on the nuclear problem of neurodevelopment may be minimal, the rest of the objectives focused on improving the quality of his relationships (and his life) if they are attainable.

Conflict of interest: No

Keywords: psychotherapy; Adoption; Asperger; attention deficit

\section{EPV0198}

Mental health care needs and access to specialized services in children with mental disorders

L. Díaz-Castro $^{1 *}$, M. Márquez-Caraveo ${ }^{2}$ and H. Cabello-Rangel ${ }^{3}$

${ }^{1}$ National Institute of Psychiatry Ramon de la Fuente Muñiz, Direction Of Epidemiological And Psychosocial Research, Mexico City, Mexico;
${ }^{2}$ Child Psychiatric Hospital "Dr. Juan N. Navarro"., Division Of Research, Mexico City, Mexico and ${ }^{3}$ Psychiatric Hospital "Fray Bernardino Álvarez"., Division Of Diagnostic Assistants, Mexico City, Mexico

${ }^{\star}$ Corresponding author.

Introduction: The prevalence of mental disorders (MD) and contribution to the global burden of disease (GBD) is greater in children (1), but they are the population with less access to mental health services (MHS) (2).

Objectives: To explore the characteristics of help-seeking and access to specialized MHS in children with MD in Mexico.

Methods: A cross-sectional study was carried out in Children's Psychiatric Hospital (HPI), through the survey method. The project was approved by the HPI Ethics Committee. Descriptive statistics for the characteristics of the sample applying measures of central tendency, Inferential statistics with t-test for differences in means between groups (girls and boys, diagnosis), and variables associated with the help-seeking to MHS. Finally, a classification method was applied through a decision tree to observe the natural behavior in the access to MHS.

Results: 456 participants. $22 \%$ female with an average age of 9.8 years $(\mathrm{SD} \pm 2.65), 72 \%$ had a diagnosis of hyperkinetic disorder (HD) and 28\% depressive disorder (DD). Medical comorbidity was

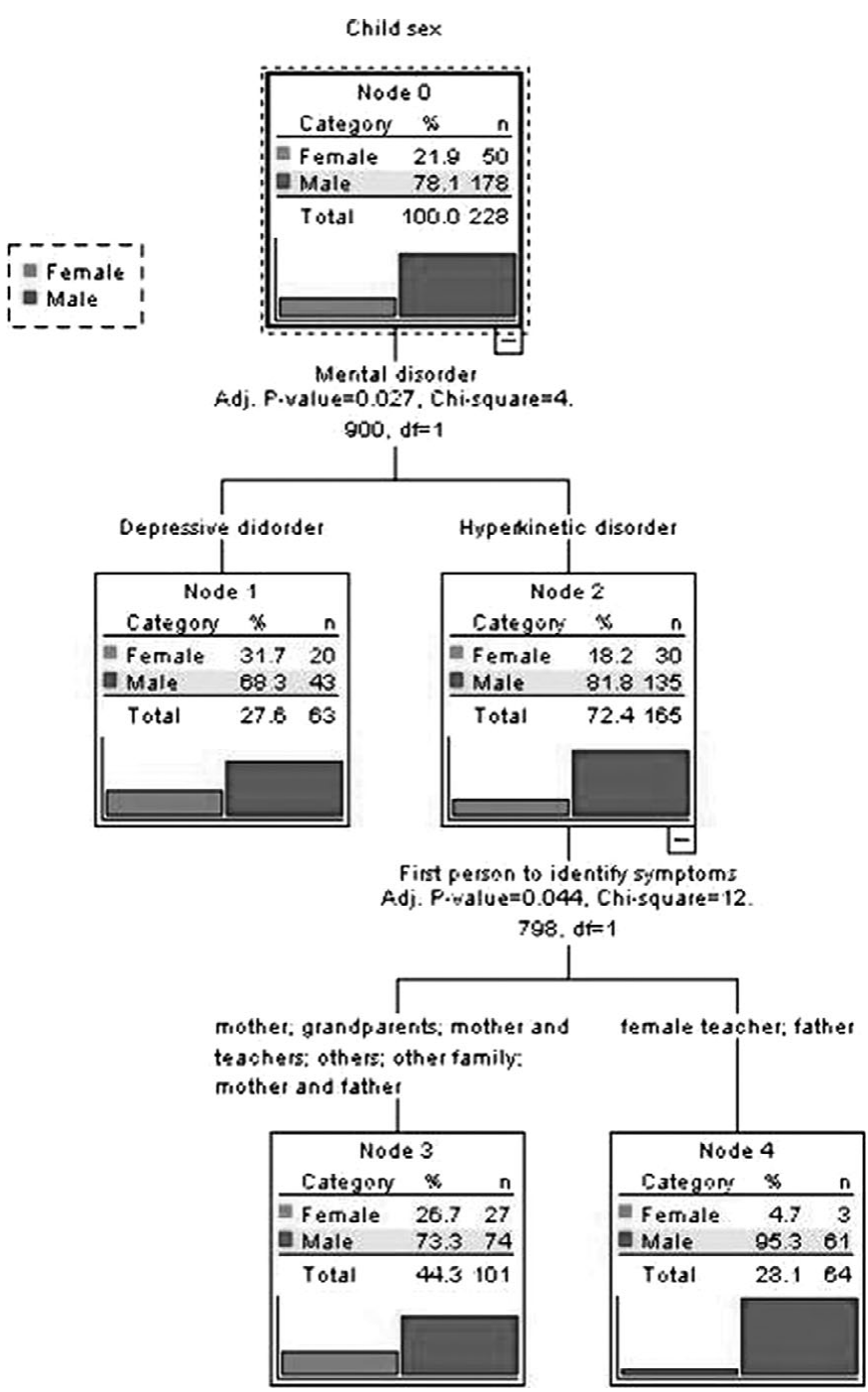


higher in girls than boys ( $26 \%$ vs $7 \%, \mathrm{X} 2=62, \mathrm{p}=.000)$. The average age to seek MHS with DD was $6.97(\mathrm{SD} \pm 2.1)$ vs $5.98(\mathrm{SD} \pm 2.3)$ in $\mathrm{HD},(\mathrm{T}=3.08, \mathrm{p}=0.003)$. The mother was the first person to notice symptoms. $43 \%$ of children received mental health-care prior to HPI. 58\% had a family with MD. (Figue 1 ).

Conclusions: The seek for MHS differs by sex, diagnosis and family, it is necessary to design mental health interventions with an emphasis on gender.

Conflict of interest: No

Keywords: Mental health needs; Access; Children; mental disorders

\section{EPV0199}

\section{Diagnostic stability in early-onset first-episode psychosis}

\section{E. Dutilh Fernandez ${ }^{\star}$ and M. Mendez Maga;a}

Hospital Virgen del Puerto, Unidad De Hospitalizacion Breve, Paraje de Valcorchero, Spain

${ }^{\star}$ Corresponding author.

Introduction: A heterogeneous group of disorders which includes different diagnoses such as schizophrenia, bipolar disorder, schizoaffective disorder, psychosis nor otherwise specified or developmental disorders with psychotic features are all included within the title of "Early onset psychosis" when they appeared prior to 18 years old. Due to this variety of psychosis-like symptoms and the variety of diagnosis, low rates of diagnostic stability is proven to last during the first two years after first psychotic episode as the symptoms changes presentation. Studies have shown that the lack of diagnostic stability still represents a pervasive difficulty in the diagnostic legitimacy of some types of early onset psychosis.

Objectives: Discuss the diagnosis of a 12 year old patient that presents whiting 5 months a psychotic episode with all possible positive symptoms (hallucinations, delusions, thoughts disorder and behaviour disorder) followed by a maniac episode.

Methods: Explained the patients' two episodes and present all the clinical reviews he has had during the time he was admitted in a child and adolescent psychiatry unit at a university hospital in Spain.

Results: As a result the patient was diagnosed Schizoaffective disorder treated with Clozapine and Lithium as he was resistant to high dose of Risperidone and Aripiprazole. Discharged completely well and asymptomatic with no positive symptoms and euthymic.

Conclusions: Diagnostic stability has been defined as the degree to which an original diagnosis at intake is confirmed during follow-up. The degree of diagnostic stability is important as regards the validity of the different diagnostic criteria which are established.

Conflict of interest: No

Keywords: Schizoaffective disorder; first-episode psychosis; psychosis; early-onset psychosis

\section{EPV0202}

\section{Relation between trauma and psychosis at transition} period (late adolescente and young adult)

\footnotetext{
E. Garcia ${ }^{1 \star}$ and P. Barredo ${ }^{2}$

${ }^{1}$ Sescam, Psychiatry, Toledo, Spain and ${ }^{2}$ Sescam, Psychology, Toledo, Spain

${ }^{\star}$ Corresponding author.
}

Introduction: It has been proved the relation between first infancy traumas (sexual or physical abuse), as independent factor, to increase the risk of developping psychosis. However in spite or recent findings and biological vulnerbality associated, its influencie in late adolescence has not been well studied. The so called transition evolutive stage is an important period of live in which many changes happen at the same time, so many changes imply a special vulnerability to stressors, and therefore to trauma. A wide definition of psychosis, following pinel or llopis statements colud be a failure to buid a flexible, adaptative identity Question is to clarify the rol of trauma and its relation to psychosis at this age in which an independent identity emerge.

Objectives: prove trauma at transition stage acts as independent risk factor to psychosis Discuss medical models vs phenomenological ones as it may proved better to reach more therapeutic targets Discuss multiintervention transdisciplinary models to improve clinical

Methods: Prospective two years long 300 patients descriptive study transition program

Results: Trauma is high represented at our sample but influenced by others factors multiintervention model reduces the prognosis factor of delayed treatment thanks to reach a high risk group in the early stages

Conclusions: We should look for a better way to clarify syndromes in order to match our speciality with medical ones, Linking to last neurofindigs which confirm pinel and llopis theories so we could achieve better results using new diagnoses and treatmets models that include the rol of factors trauma and developmental

Conflict of interest: No

Keywords: trauma; psychosis; Transition; Multiintervention

\section{EPV0222}

\section{Generalized problematic internet use, use of social} networks, and appearance schemas in late adolescence

\section{B. Maia $^{1 \star}$, H. Moreira ${ }^{2}$, A.T. Pereira ${ }^{3}$ and A. Macedo ${ }^{4}$}

${ }^{1}$ Universidade Católica Portuguesa, Centre For Philosophical And Humanistic Studies, Braga, Portugal; ${ }^{2}$ University of Coimbra, Center for Research In Neuropsyhology And Cognitive Behavioral

Intervention, Coimbra, Portugal; ${ }^{3}$ Faculty of Medicine, University of Coimbra, Institute of Psychological Medicine, Coimbra, Portugal and ${ }^{4}$ University of Coimbra, Faculty of Medicine, Department of Psychological Medicine, Coimbra, Portugal

${ }^{\star}$ Corresponding author.

Introduction: Research on the association between internet use and concerns about body image is still scarce.

Objectives: To explore the associations between generalized problematic internet use, number of social networks, and appearance schemas.

Methods: 216 Portuguese late adolescents (89.3\% females), with a mean age of 18.62 years old ( $\mathrm{SD}=.488$, range: $18-19)$ filled in an internet patterns of use questionnaire, the Generalized Problematic Interne Use Scale 2 (GPIUS2) and The Appearance Schemas Inventory-Revised (ASI-R).

Results: A total of $99.6 \%$ of the students use social networks. Subjects were divided into three groups (group1: 1-2 social networks; group 2: 3 social networks, and group 3: $>3$ social networks). There was a statistically difference in Motivational Salience scores (ASI-R) for the three groups $[\mathrm{F}(2,503)=6.0, \mathrm{p}=.003]$. Post-hoc comparisons indicated that the mean score for group $3(\mathrm{M}=28.29$, 
$\mathrm{SD}=4.95)$ was significantly different from group $1(\mathrm{M}=26.69$, SD $=4.55)$, and from group $2,(\mathrm{M}=26.87, \mathrm{SD}=4.95)$. Generalized problematic internet subscales (Mood Regulation, Self-Deficient Regulation, and Negative Consequences) and total score were significantly correlated with both dimensions of ASI-R: SelfEvaluation Salience (coefficients varied from $r=.31^{\star}$ to $r=.47^{\star}$ ) and Motivational Salience (from $r=.14^{\star}$, to $r=.31^{\star}$ ).

Conclusions: Generalized problematic internet use and the number of social networks are associated with adolescent's cognitivebehavioural investment in one's own appearance. This study was carried out under the strategic project of the Centre for Philosophical and Humanistic Studies (CEFH) UID/FIL/00683/2019, funded by the FCT.

Conflict of interest: No

Keywords: Problematic Internet Use; appearance schemas; use of social networks; Late Adolescence

\section{EPV0223}

Associations between social anxiety and avoidance, attachment styles and parental marital status, in late adolescence

B. Maia $^{1 \star}$, C. Coelho ${ }^{2}$, M. Marques ${ }^{3}$ and F. Carvalho ${ }^{4}$

${ }^{1}$ Universidade Católica Portuguesa, Centre For Philosophical And Humanistic Studies, Braga, Portugal; ${ }^{2}$ The Catholic University of Portugal, Faculty of Philosophy And Social Sciences, Braga, Portugal; ${ }^{3}$ Coimbra Hospital and University Centre, Clinical Psychology, Coimbra, Portugal and ${ }^{4}$ Espaço Psicológico - Consultório de Psicologia, Consultório De Psicologia, Coimbra, Portugal

${ }^{\star}$ Corresponding author.

Introduction: The relation between insecure general attachment and social anxiety has long been established.

Objectives: To explore the associations between social interaction and performance anxiety and avoidance, attachment styles, and parental marital status.

Methods: 146 Portuguese adolescents, with a mean age of 18.99 years old $(\mathrm{SD}=.848$; range: $18-20)$, filled in the Social Interaction and Performance Anxiety and Avoidance Scale and the Experiences in Close Relationships-Relationship Structures Questionnaire.

Results: Distress/Anxiety was correlated with avoidance attachment to mother and father ( $\mathrm{rs}=.17^{\star}, \mathrm{p}=.04$; $\mathrm{rs}=.18^{\star}, \mathrm{p}=.03$ ), to anxious attachment to romantic partner ( $\mathrm{rs}=.21^{\star}, \mathrm{p}=.01$ ), and to anxious and avoidance attachment to best friend ( $\mathrm{rs}=.25^{\star}, \mathrm{p}=.00$; $\left(\mathrm{rs}=.17^{\star}, \mathrm{p}=.035\right)$. Avoidance was significantly correlated with avoidance to father and to romantic partner $\left(\mathrm{rs}=.18^{\star}, \mathrm{p}=.03\right)$, and to anxious and avoidance attachment to best friend ( $\mathrm{rs}=.21^{\star}, \mathrm{p}=$ .009 ; $\mathrm{rs}=.18^{\star}, \mathrm{p}=.03$ ). A significant difference was found in avoidance attachment to father $\mathrm{X}^{2}=10.246(4, \mathrm{n}=146), \mathrm{p}=$ .036 , by parental marital status, with the adolescents with single/ divorced parents presenting a higher mean score $(\mathrm{Md}=111.10$; $\mathrm{Md}$ $=82.93$ ) than the other groups.

Conclusions: Distress/anxiety seems to be associated with more close relationships, and a single/divorced status with Avoidance. Longitudinal studies are needed to explore if insecure attachment to parents predicts insecure extra-familiar attachment, and to explore the long-term effects of parental marital status.

Conflict of interest: No

Keywords: parental marital status; attachment; Late Adolescence; social anxiety and avoidance

\section{EPV0226}

\section{The influence of new technologies in adolescents and young people}

R. Molina Cambra*, M. García-Poggio Fernández-Renau, A. Muñoz Domenjó, E. Ramos García, Â. Martínez Fernández, F.L. Bianchi Ramos, R. Sagarra Arruego, M. Ortega Moreno and M. Hernández Barrera

Hospital Universitario de Móstoles, Psiquiatría, Móstoles, Madrid, Spain

${ }^{\star}$ Corresponding author.

Introduction: Information and Communication Technologies (ICT) have changed lifestyle of most of the population, modifying how people relate to each other. The impact has been remarkable in all ages, but it highlights in children, adolescent and young adults, where virtual world has as much reality as face-to-face relationships and represents a way of fleeing from a daily routine and getting lost in a fantasy universe.

Objectives: To review the current situation of scientific knowledge on addiction and abuse of new technologies by adolescents and young people, in order to describe and analyze the dangers entailed and to incise the importance of prevention strategies.

Methods: We review recent literature using PubMed and MeshDatabase, related to several patients of our Child Psychiatry Outpatient Clinic.

Results: During adolescence there is a process of personal identity acquisition, with both physical and psychological changes that make them emotionally vulnerable. Therefore, the probability of suffering from an addiction to new technologies increases at these ages. Behavioral addictions exhibit similar characteristics of established addictions: inability to self-control, psychological dependence and personal, family and social misadjustments.

Conclusions: Given the high prevalence of these type of addictions in our society, we must value the need to apply psychoeducational methods both in the family and at school. The aim of the treatment for new technologies addiction, unlike other addictions, cannot be total abstinence, but rather controlled use. The gold standard treatment appears to be stimulus control and gradual exposure, followed by a cognitive-behavioral intervention in relapse prevention.

\section{Conflict of interest: No}

Keywords: adolescents; young people; new technologies; behavioral addictions

\section{EPV0238}

\section{Common aspects of behavioral disorders and related diagnoses.}

V. Quero-Palomino*, I. Diaz-Quero, V. Muñoz-Martínez, B. MataSaenz, A. Gomez-Peinado and R. Torralba-Viorreta

Hospital General Universitario de Ciudad Real, Psychiatry, Ciudad Real, Spain

${ }^{\star}$ Corresponding author.

Introduction: Behavioral disorders (BD) constitute some of the most prevalent diagnosis in children and adolescents' mental health. Investigation has been increased because of this high frequency and the need of an early diagnosis and searching the most efficient therapeutic alternatives. Besides the symptoms, they cause 
an important dysfunction in different contexts, especially in the family environment.

Objectives: To analyze the common characteristics of BD in adolescents and relate to a bibliographic review.

Methods: A retrospective, descriptive study was performed. It included BD cases, according to DSM- 5 criteria, admitted at Child and Youth Hospitalization Unit. We selected ten patients who met the inclusión criteria. Data and variables collected were: age, sex, admission and discharge diagnosis, hospitalization time, previous and discharge treatment, use of psychoterapeutic approach focused on emotion management and family intervention.

Results: About $80 \%$ of the sample were males. Mean age was 15.6 (standard deviation 1.35). All of them were diagnosed at admission of behavioral alterations. Mean hospitalization time was 3.9 weeks (standard deviation 1.85). Diagnoses at discharge included: neurodevelopment disorders, psychotic disorders, anxiety and depression disorders. The main drugs used were aripiprazole, olanzapine, risperidone, guanfacine and clonazepam. Emotional management strategies and family interventions were applied to all patients.

Conclusions: Behavioral disorders require a specific approach to the underlying disease beyond symptomatic control. This one will change the prognosis and the need of pharmacotherapy. Atypical antipsychotics are the most used drugs. The family impact makes essential its management as part of the treatment both at the hospital and outpatient level.

Conflict of interest: No

Keywords: family intervention; pharmacotherapy; adolescents; Behavioral disorders

\section{EPV0242}

\section{Avoidant restrictive food intake disorder: an illustrative case example}

V. Taillefer Aguanell ${ }^{1 \star}, F$. Barquero $\mathrm{Paz}^{2}$, A. Más Villaseñor ${ }^{3}$, M. Plaza Yuste ${ }^{4}$ and A. Muñoz Domenjó

${ }^{1}$ Hospital Universitario Guadalajara, Psiquiatría, Guadalajara, Spain; ${ }^{2}$ Hospital Universitario Badajoz, Psiquiatría, Badajoz, Spain; ${ }^{3}$ Hospital Nuestra señora de Sonsoles Ávila, Psiquiatría, Ávila, Spain; ${ }^{4}$ Hospital Central de la Defensa Gómez Ulla, Psychiatry And Mental Health Department, Madrid, Spain and ${ }^{5}$ Hospital universitario móstoles, Psiquiatría, Madrid, Spain

${ }^{\star}$ Corresponding author.

Introduction: ARFID is a new Psychiatric Disorder included in DSM-V. People with ARFID have underlying biological traits that initially made their eating habits a logical choice. This behavior is often associated with unintentional weight loss, failure to achieve expected weight gain or height, nutritional deficiency, impact on daily functioning, and even dependence on enteral feeding. Objectives: This case of a 13-year old girl with ARFID illustrates key issues in diagnosis and treatment planning. At the age of two, she experienced an allergic reaction to peanuts and two years later she suffered severe diarrhea. After both episodes she has been very skeptical to food. In order to solve these problems certain foods were eliminated from her diet. Nevertheless, the patient continued to eat little justifying it with ongoing digestive discomfort. Due to this attitude she lost weight and had to receive pediatric treatment. Since no improvement was made, the patient was then referred to the eating disorders psychiatric specialists for one year. However, hospital admission has been performed given her weight stagnation. Methods: During admission, long term bad eating habits are revealed, accompanied by regressive reactions. These behaviors have required psychotherapeutic and pharmacological interventions to eliminate the symptoms

Results: After hospitasization, the BMI has been increased and food behaviors have been modified in order to continue treatment in the day hospital.

Conclusions: ARFID is a heterogeneous diagnosis with diverse etiologies. Thus, identification of best practice guidelines is challenging. Existing literature promotes a multidisciplinary care approach that integrates behavioral, cognitive behavioral, and family-based interventions.

\section{Conflict of interest: No \\ Keywords: ARFID; Feeding; disorder; childhood}

\section{EPV0251}

\section{Is there a specific developmental organization of graphomotor gesture in school aged children with handwriting disabilities?}

\section{Lopez ${ }^{1}$, H. Brunel ${ }^{2}$ and L. Vaivre-Douret ${ }^{3 \star}$}

${ }^{1}$ Faculty of Medicine, University of Paris, Paris Descartes ; INSERM Unit1178/1018-CESP, Necker-Enfants Malades University Hospital, AP-HP, France, Department of Child Psychiatry, Paris, France, Paris, France; ${ }^{2}$ INSERM Unit1178/1018-CESP, Necker-Enfants Malades University Hospital, AP-HP ; Department of Engineering of Automatic Systems and Industrial Computing, Polytecnic University of Ca, Department of Child Psychiatry, Paris, France, Paris, France and ${ }^{3}$ Faculty of Medicine, University of Paris, Paris Descartes ; INSERM Unit1178/1018-CESP, Necker-Enfants Malades University Hospital, AP-HP, France ; France University Institute (IUF), Department of Child Psychiatry, Paris, France, Paris, France ${ }^{*}$ Corresponding author.

Introduction: The handwriting gesture organization is poorly studied in international researches on handwriting. We have previously shown that there is a maturative developmental organization of the handwriting gesture in school aged typical children (Lopez et al., 2019).

Objectives: The aim of our study is to examine the handwriting gesture organization in children with handwriting disabilities.

Methods: 31 right-handed children (6-11 years old) with handwriting disabilities, in primary school, were included in the study and compared to 135 typical children. The articular (shoulderelbow-wrist) and segmental (arm-forearm-hand) organization of the handwriting arm was observed in an ecological way, in a paperpencil situation, thanks to two cameras (3D reconstruction) filming the child during of a copy of cycloid loops with the dominant hand. Results: The articular / segmental variables correlate with each other to form patterns organization of the graphomotor gesture in children with handwriting disabilities. This gestual organizations are statistically significantly different from those of typical children $(\mathrm{p}<0.01)$, highlighting less mature organization than younger typical children and more variability of organizations in children with handwriting disorders.

Conclusions: There is an immaturity of the segmental organization of the graphomotor gesture in children with handwriting disabilities in comparison with typical children. This specific sensori-motor organization can participate in the symptomatology of handwriting disorders with kinetic mesures and must be considered in the understanding of neurodevelopmental process for the diagnosis of handwriting disorders. Remediation strategies should take account the developmental organization of handwriting gesture. 
Conflict of interest: No

Keywords: handwriting gesture; child; development; handwriting disabilities

\section{EPV0266}

\section{The mental health of minors residing in centers}

\section{C.L. Gómez González ${ }^{1 \star}$, I.P. Imma ${ }^{2}$ and C.F. Marta ${ }^{2}$}

${ }^{1}$ Hospital Sant Joan de Déu of Barcelona, Child And Adolescent Psychiatry And Psychology Department, Esplugues de Llobregat, Barcelona, Spain and ${ }^{2}$ Sant Joan de Deu Hospital, Child/youth Mental Health Centre, Barcelona, Spain

${ }^{\star}$ Corresponding author.

Introduction: There are special residential programs for minors that are guarded by the government and have severe behavioral problems. Adolescents who suffer from severe emotional or behavioral disorders are at an increased risk for negative outcomes: academic dropouts, drug problems, difficulty with their peers, unemployment, and problems with authority.

Objectives: The purpose of this study is to examine the mental health of the minors that reside in one center to further understand what the intervention is needed.

Methods: We gathered 58 minors that were living at de center from November 2018 to October 2019 and that was assessed by psychiatry. DSM-5 criteria were used to evaluate.

Results: They were all males, ages 12 to 18 . The most frequent diagnosis was attention deficit hyperactivity disorder (37.93\%), followed by oppositional defiant disorder (20.68\%) and conduct disorder (10.34\%). More than 50\% received more than one diagnosis. Other diagnoses included intellectual disability (15.52\%), psychosis $(8.62 \%)$ and affective disorders (8.62\%). 21 of the minors didn't take pharmacological treatment $(36.21 \%)$. The most common treatment was antipsychotics, followed by stimulants and other ADHD medication.

Conclusions: We found coherence with the literature, being the behavioral diagnosis more frequent in this type of patient. The diagnosis added to the high prevalence of antisocial conduct and prevalence of drug abuse can explain some of the problems the adolescents had at home or in other less structured fostered centers. Something to take in mind is the low ratio of pharmacology treatment, which could indicate that other psychological and educational interventions are more effective in these patients.

Conflict of interest: No

Keywords: fostercare; behavioral problems; residential centers; adolescents

\section{EPV0282}

Some gifted children are diagnosed with developmental coordination disorder (DCD) but have they identical clinical features compared to typical children with DCD?

L. Vaivre-Douret $^{1 \star}$, S. Hamdioui ${ }^{2}$ and A. Cannafarina ${ }^{2}$

${ }^{1}$ Faculty of Medicine, University of Paris, Paris Descartes; INSERM UMR 1178/1018-CESP, and University Institute (IUF), Department of Child Psychiatry, Necker-enfants-malades University Hospital, Ap-hp, Paris, France and ${ }^{2}$ Faculty of Medicine, University of Paris, Paris Descartes, INSERM Unit 1178/1018-CESP, Department of Child Psychiatry, Necker-enfants-malades University Hospital, Ap-hp,

Paris, France

${ }^{\star}$ Corresponding author.
Introduction: Normal distribution considers a high Intellectual Quotient (HIQ) at 120 (8.9\%) and gifted children (GC) beginning at 130 IQ (2.2\%). Some of them also happen to have DCD, but are rarely diagnosed for it.

Objectives: to explore IQ in a sample of DCD and to analyze clinical features regarding HIQ and GC compared to typical children with DCD.

Methods: In a sample of 74 children (9 years 1 month, $2.7 \mathrm{Sd}$ ) diagnosed with DCD according to DSM-5 without comorbidity were split into two paired of DCD with $19 \mathrm{HIQ}$ and 19 GC matched with DCD typical group $(90<\mathrm{IQ}<110)$. The sample was divided into 3 pure subtypes of DCD according previous studies (ideomotor (IM), visual-constructive (VC), visual-spatial (VS)), and a mixed DCD. All children were assessed with neuropsychomotor, neuropsychological and neurovisual tests.

Results: HIQ and GC did not significantly differ from DCD typical group regarding to VS and mixed DCD. However, they are significantly less IM $(\mathrm{p}=.03)$ and $\mathrm{VC}(\mathrm{p}=.02)$. In addition, we have found in HIQ and GC significant correlations between IM and executive functions $(\mathrm{r}=.519 \mathrm{p}<.05=.001)$, also between Khos cubes test and $\mathrm{VC}(\mathrm{r}=.815, \mathrm{p}<.05)$. Regarding neuropsychomotor assessment, these two groups answer significantly $(\mathrm{p}=.05)$ correctly about object's spatial orientation but not for body integration of spatial orientation. Conclusions: HIQ and GC with DCD have similar clinical features compared to DCD typical group. However, HIQ and GC with DCD seem to use more mental representations and planning which appear as a good cognitive approach to compensate ideomotor or visual-constructional DCD.

\section{Conflict of interest: No}

Keywords: high intellectual quotient; IQ; gifted children; developmental coordination disorder

\section{Classification of mental disorders}

\section{EPV0284}

\section{The schizo-obsessive spectrum. About a clinical case.}

A. Rodríguez-Quiroga ${ }^{1 *}$, P. $\mathrm{Nava}^{2}$, A.M. Matas Ochoa ${ }^{1}$, R. Martínez De Velasco ${ }^{1}$, C. Banzo ${ }^{2}$ and F. Mora ${ }^{2}$

${ }^{1}$ Hospital Universitario Infanta Leonor, Psychiatry, Madrid, Spain and ${ }^{2}$ Hospital Infanta Leonor, Psychiatry, Madrid, Spain

${ }^{*}$ Corresponding author.

Introduction: The coexistence of psychotic symptoms and obsessive-compulsive symptoms (OCS) has been known since the 19th century, with a high prevalence of both OCS (25\%) and obsessive compulsive disorder (OCD) (12\%) in patients with schizophrenia.

Objectives: The main objective is to assess the validity of the schizoobsessive disorder as a diagnostic entity for those patients who show symptoms of schizophrenia and OCD.

Methods: We present the case of a 61-year-old male with a diagnosis of schizophrenia from the age of 28 and obsessive-compulsive symptoms in the last 5 years.

Results: The patient has important negative symptomatology, with ideoaffective impoverishment, short speech, abulia, motor slowing, poor social skills, abandonment of self-care, tendency to isolation, obsessive thoughts and compulsions of doubt and verification. $\mathrm{He}$ describes the presence of magical thinking, having to leave the doors opened, with the fear of bad things happening instead, he 
washes hands ten times a day to get rid of the negative energy of the objects, leaves the shoes in the room always in the same way and in the same place to prevent something bad from happening. He also maintains a delusional ideation of unstructured, chronic damage, without behavioral repercussion. Complementary tests (blood test and cranial CT) were normal.

Conclusions: The differential diagnosis is made between schizoobsessive disorder and comorbid schizophrenia with OCD. He is being treated with clozapine, which has shown an exacerbation of obsessive symptoms, which is why amisulpride and sertraline are added. The schizo-obsessive disorder has clinical, neurobiological and neurocognitive features distinct from both schizophrenia and OCD.

\section{Conflict of interest: No}

Keywords: schizophrenia; magical thinking; obsessive-compulsive disorder; clozapine

\section{EPV0285}

The IQ profile (homogeneous versus heterogeneous) in children with high intellectual potential (HIP): what are the links with some emotional, interactional and neurodevelopmental disorders?

\author{
L. Vaivre-Douret ${ }^{1 \star}$ and P. Planche ${ }^{2}$ \\ ${ }^{1}$ Faculty of Medicine, University of Paris, Paris Descartes ; INSERM \\ UMR 1178/1018-CESP, Necker-Enfants-Malades University Hospital, \\ AP-HP, France University Institute (IUF), Department of Child \\ Psychiatry, Paris, France and ${ }^{2}$ Faculty of letters and Human Sciences, \\ Brest University, Laboratory Cread (ea 3875), Brest, France \\ ${ }^{*}$ Corresponding author.
}

Introduction: In clinical practice, some emotional, interactional and neurodevelopmental disorders are often observed in children with high intellectual potential (HIP).

Objectives: The homogeneity of the cognitive profile often accompanies a good daily adaptation, so we hypothesize that these disorders are more likely to affect children with HPI with a heterogeneous profile than those with a homogeneous Wechsler-scale profile.This study aims to verify these assumptions.

Methods: All children > 130 IQ, 7 to 15 years old were assessed with the Wechsler Intelligence scale for children. We have established two groups, homogeneous IQ profile group (HOIQ, $n=13$ ) and heterogeneous IQ profile group (HEIQ, $n=24$ ). The heterogeneous profile is defined by a difference of at least 23 points between the higher and the lower index score. It was also administrated BarnCohen's Empathizing Quotient (EQ) and Autism spectrum Quotient (AQ) tests, theMultiscore Depression Inventory for Children (MDI-C), the Beery Developmental Test of Visual-Motor Integration (VMI), and a motor coordination test (jump) of the VaivreDouret NeuroPsychoMotor functions battery (NP-MOT).

Results: We found significative differences $(\mathrm{p} \leq .05)$ between the two groups: a higher score to the AQ test, a lower score of visuomotricity and motor coordination, higher scores of depression symptoms, in HEIQ group compared to HOIQ group.

Conclusions: Our results confirm the higher frequency of disorders in the heterogeneous profile group than in the homogeneous profile group. They could also provide indicators for making a differential diagnosis between the heterogeneous HPI group and people with high-level autism associated with HIP.

Conflict of interest: No

Keywords: High intellectual potential child; homogeneous IQ profile; heterogeneous IQ profile; Neurodevelopmental disorders

\section{EPV0287}

\section{Dimensional analysis of adolescent attention-deficit/ hyperactivity disorder}

P. Fernández-Martín ${ }^{1 \star}$, J.J. León ${ }^{1}$, R. Rodríguez-Herrera ${ }^{1}$, R. Cánovas ${ }^{2}$, A. Martínez De Salazar ${ }^{1,3}$, L. Cobos-Sánchez ${ }^{1,4}$, F. Sánchez-Santed ${ }^{1,2}$ and P. Flores ${ }^{1,2}$

${ }^{1}$ University of Almería \& Health Research Centre, Department of Psychology, Faculty of Psychology, Almería, Spain; ${ }^{2}$ InPaula, Institute of Child Neurorehabilitation, Department of Neuropsychology, Almería, Spain; ${ }^{3}$ Torrecárdenas University Hospital, Child And Adolescent Mental Health Unit, Almería, Spain and ${ }^{4}$ Sol de Portocarrero High School, Department of Educational Guidance, Almería, Spain ${ }^{\star}$ Corresponding author.

Introduction: In recent years, we are witnessing a crisis in diagnostic classification systems. Prevalence of mental health disorders has increased considerably, among various reasons, because traditional diagnostic criteria are not sensitive enough to detect their great heterogeneity. Attention-Deficit/Hyperactivity Disorder (ADHD) is one of the most prevalent mental health disorders, whose dimensional character has been pointed out.

Objectives: The aim of this study was to improve ADHD diagnosis by identifying ADHD specific dimensional profiles.

Methods: 100 adolescents (12-16 years old) were recruited from the local community, $45 \mathrm{ADHD}$ and 55 controls, and were assessed with AULA Nesplora, a virtual-reality Continuous Performance test (CPT) specially designed for the assessment of attentional processes, impulsivity, processing speed and motor activity level. Hierarchical and non-hierarchical cluster analysis were performed to identify new homogeneous groups based on specific behavioural symptoms. One-way ANOVA was used to compare between-group differences in performance.

Results: Five dimensional profiles were obtained: two normative groups and three groups with different impairments that don't match with the traditional ADHD subtypes. These last three groups shared difficulties in selective and sustained attention but differed in the presence and severity of impulsive and/or hyperactive behaviours. Conclusions: These findings represent an advance in the understanding of ADHD symptoms, highlight the need to update diagnostic criteria and could be useful to design most effective intervention strategies based on specific behavioural dimensions. Funding: Ministerio de Ciencia, Innovación y Universidades and Fondo Europeo de Desarrollo Regional (MINECO-FEDER) [Grant numbers: PSI2017-86847-C2-1-R and PGC2018-099117-B-C21].

\section{Conflict of interest: No}

Keywords: Dimensional diagnosis; attention; ADHD; inhibitory control

\section{EPV0289}

\section{A critical overview of the schizophrenia-obsessive- compulsive disorder interphase}

\section{J.E. Ibáñez Vizoso*, B. Rodado León and M.D. Saiz González}

Hospital Universitario Clínico San Carlos, Instituto De Psiquiatría Y Salud Mental, Madrid, Spain

${ }^{\star}$ Corresponding author.

Introduction: The presence of obsessive-compulsive symptoms (OCS) in schizophrenia and of psychotic features (from lost of insight to real psychotic symptoms) in some OCD has long been 
recognized. Bleuler and Mayer Gross considered chronic obsessives as schizophrenics; Ey and Huber described obsessive symptoms as prodromes, and Leonhard described the anancastic forms of systematic schizophrenias. Nowadays, OCD and schizophrenia remain different diagnostic categories; the high comorbidity of both of their symptoms has driven the creation of constructs such as a schizo-obsessive spectrum and a schizo-obsessive subtype of schizophrenia.

Objectives: To review the association between Schizophrenia and OCS/OCD and to discuss the rationale for a schizo-obsessive spectrum/subtype of schizophrenia.

Methods: A comprehensive literature search was conducted via pubmed, using the terms "Schizoobsesive", "Schizophrenia" and "Obsessive-Compulsive Disorder" or"Obsessive compulsive symptoms".

Results: The term "schizoobsessive disorder" was coined in 1993, including in it the schizophrenia-OCS comorbidity, and severe OCD patients with lost of insight or schizotypic traits. Some authors have later approached the issue from a spectrum or interface perspective. Transition from OCD to Schizophrenia is uncommon. Schizophrenia frequently presents with OCS, which are similar to those of OCD, may present along the whole course of disease and relate to a poorer prognosis. Low-insight OCD seems a more severe form of OCD, while OCD-schizotypy comorbidity may represent a different subgroup. Antipsychotics may induce OCS.

Conclusions: Given the complex association of Schizophrenia and OCD/OCS, a careful assessment is mandatory in order to ascertain the primary diagnosis and the direction of the association. Therapeutic issues must be taken into account.

Conflict of interest: No

Keywords: obsessive-compulsive symptoms; schizophrenia; schizoobsesive; Obsessive-Compulsive Disorder

\section{Comorbidity / dual pathologies}

\section{EPV0305}

\section{Ticking time-bomb}

C. Loeck De Lapuerta ${ }^{1 *}$, M. Rodriguez De Lorenzo ${ }^{2}$, C. Carrajo Garcia $^{3}$ and E. Alonso Sánchez ${ }^{4}$

${ }^{1}$ RAMON Y CAJAL UNIVERSITY HOSPITAL, Psychiatry, MADRID, Spain; ${ }^{2}$ Hospital Universitario Ramon y Cajal, Psychiatry, Madrid, Spain; ${ }^{3}$ HOSPITAL UNIVERSITARIO RAMON Y CAJAL, Psychiatry, MADRID, Spain and ${ }^{4}$ HOSPITAL UNIVERSITARIO RAMON Y

CAJAL, Psychiatry, Madrid, Spain

${ }^{\star}$ Corresponding author.

Introduction: Brugada syndrome is an inherited disease characterized by a coved-type ST-segment elevation in the right precordial leads and increased risk of sudden cardiac death (SCD), in the absence of structural abnormalities. We present the case report of a 20 -year-old male who was diagnosed with paranoid schizophrenia at the age of 17. During his assessment at the hospital he presented an EKG with alterations compatible with brugada syndrome. In addition, the patient consumes cannabis and alcohol with a daily abuse pattern wich is really harmful for both conditions.

Objectives: The case was difficult to handle due to the possible cardiological damage derived from the use of antipsychotics and psychiatric drugs. In this poster we review the safest psychiatric drugs for the management of these patients and those that we should avoid.

Methods: Representative case report. Research in pubmed about Brugada Syndrome and Psycotropic drugs. Research in http://www. brugradadrugs.org/ Key words: Brugada, schizophrenia, antipsychotic drugs, dual patology

Results: Psycotropic drugs that you should avoid completely in Brugada syndrome: bupropíon, carbamazepina, ciamemazina, clotiapina, dosulepina, doxepina, fluoxetina, fluvoxamina, imipramina, lamotrigina, maprotilina, paroxetina, perfenazina, fenitoína, tioridazina Psycotropic drugs that you should avoid as possible in Brugada syndrome: amitriptilina, clomipramina, desipramina, litio, loxapina, nortriptilina, oxcarbazepina, trifluoperazina

Conclusions: The patient was treated with aripiprazol which was well tolerate, safe and effective.

\section{Conflict of interest: No}

Keywords: brugada; schizophrenia; dual patology; antipshycotic drugs

\section{EPV0313}

Stabilization of a patient with a major depressive sindrome with psychotic and obsessive symptoms and hiperthyroidism

\section{J. Vilas Boas ${ }^{\star}$ and S. Torres}

Centro Hospitalar Barreiro Montijo, Psychiatry And Mental Health, Barreiro, Portugal

${ }^{\star}$ Corresponding author.

Introduction: This is the clinical case of a patient that went through thyroidectomy, was doing hormonal replacement therapy, while being treated for a major depressive disorder. When he knew he was going to be a father he underwent through great stress and run away into the woods and took no endocrinological or psychiatric medication for 6 days. He was then hospitalised.

Objectives: The objective is to correlate the clinical presentation of this patient at ER admission with the cessation of therapeutic intake.

Methods: He was stabilized with 100mg of Sertraline at breakfast, $100 \mathrm{mg}$ of Lamotrigine at lunch and dinner, $5 \mathrm{mg}$ of Aripiprazol at breakfast, $300 \mathrm{mg}$ of Quetiapine before bed, $1 \mathrm{mg}$ of Clonazepam at breakfast and $2 \mathrm{mg}$ at bedtime and $125 \mathrm{mg}$ of Levotiroxine before breakfast.

Results: Initially the patient referred to himself as a cold blooded person, without feeling and affection for others including his pregnant wife, friends or family. After 10 days of treatment he gained insight for his cathastrophic delirium and verbalized that he actually loves his wife and about to be born daughter but the pressure of being about to be a father made him runaway and escape realitiy impulsively - without taking medication with him - beacause it was overwhealming.

Conclusions: The patient is still being studied and we want to correlate the possible interference of the absense of hormonal therapy in a patient that went through thiroydectomy with this psychiatric case.

\section{Conflict of interest: No}

Keywords: Depressive; Obessive; Psychotic; Hiperthyroidism 


\section{EPV0338}

\section{Is there a role for aripiprazole in alcohol use disorders?}

R. Gasparinho ${ }^{1 \star}$, V. Nogueira ${ }^{2}$, A. Alho ${ }^{1}$, N. Santos ${ }^{1}$, M. Martins ${ }^{1}$, N. Fernandes ${ }^{1}$, L. Ferreira ${ }^{1}$ and J. Teixeira ${ }^{3}$

${ }^{1}$ Hospital Distrital de Santarém, Psychiatry And Mental Health, Santarém, Portugal; ${ }^{2}$ Centro Hospitalar Psiquiátrico de Lisboa, Psiquiatria, Lisboa, Portugal and ${ }^{3}$ Centro Hospitalar Psiquiátrico de Lisboa, Alcoologia E Novas Dependências (and), Lisboa, Portugal ${ }^{\star}$ Corresponding author.

Introduction: Aripiprazole, an atypical antipsychotic that is a dopamine and serotonin receptor partial agonist is currently approved for the treatment of schizophrenia, bipolar disorder and treatment-resistant major depression. It is also commonly prescribed for disorders associated with problems with impulse control. Recent evidence showed that dopaminergic mechanisms are involved in motivation, reward, and reinforcement of substance abuse. Given this, Aripiprazole is a potential candidate for treatment of different types of substance dependence, including Alcohol Use Disorders (AUD).

Objectives: To review the role of aripiprazole as a therapeutic agent in AUD.

Methods: Pubmed search with MeSH terms: "Aripiprazole”, “Alcoholic Intoxication", "Alcoholism”. Search was restricted to articles written in English, without temporal limitation. Total of 27 results; 22 articles selected.

Results: Aripiprazole has been proved to be safe and well tolerated, even among those with active drinking. It has been associated with a possible modulation of circuits associated with alcohol reward and craving, therefore posing as a possible therapeutic agent in the promotion of alcohol abstinence and reduction of alcohol seeking behaviour, particularly in those with high impulsivity, alcoholrelated anxiety/low mood and anhedonia.

Conclusions: Given aripiprazole's pharmacodynamic signature it might prove to be beneficial for conditions in which dysregulation of reward and impulsivity, among them AUD, are a core feature. Scientific research seems promising, nevertheless due to the current lack of large randomized controlled studies a definitive conclusion cannot be drawn for the role of Aripiprazole in AUD.

Conflict of interest: No

Keywords: aripiprazole; alcoholism; Alcohol Use Disorders

\section{Consultation liaison psychiatry and psychosomatics}

\section{EPV0355}

\section{Cannabinoid hyperemesis in liaison psychiatry. Case report.}

M. Rodriguez De Lorenzo ${ }^{1 *}$, E. Alonso Sánchez ${ }^{1}$, C. Carrajo Garcia $^{2}$, C. Loeck De Lapuerta ${ }^{3}$, A. Correa ${ }^{4}$, A. Guillama Henriquez ${ }^{1}$ and P. Del Sol Calderón ${ }^{5}$

${ }^{1}$ Hospital Universitario Ramon y Cajal, Psychiatry, Madrid, Spain; ${ }^{2}$ HOSPITAL UNIVERSITARIO RAMON Y CAJAL, Psychiatry, MADRID, Spain; ${ }^{3}$ RAMON Y CAJAL UNIVERSITY HOSPITAL, Psychiatry, MADRID, Spain; ${ }^{4}$ Ramon y Cajal Hospital, Psychiatry, Madrid, Spain and ${ }^{5}$ HOSPITAL UNIVERSITARIO PUERTA DE HIERRO MAJADAHONDA, Psychiatry, MADRID, Spain ${ }^{\star}$ Corresponding author.
Introduction: 24 -year-old patient who uses cannabis at a moderate dose every diary who, after giving up, presents a withdrawal picture consisting of incohertitible vomiting for 15 days in the ER.

Objectives: The aim of this case report is to present a case in which a cannabinoid hyperemesis can improve with hot showers.

Methods: Case report and literature review

Results: The patient is presented in the ER with a vomiting picture of 5 days of evolution. Despite the volume replacement and administration of drugs for 48 hours, the patient remains dehydrated and does not tolerate orally, so it is decided to enter Internal Medicine. Haloperidol is tested up to $15 \mathrm{mg}$ daily and Diazepam $30 \mathrm{mg}$ daily without success. Before the improvement of the picture and difficulties in handling, Link Psychiatry is notified. Acute psychopathology is ruled out at that time, it is recommended to continue with the guideline, and hot showers on demand are added to the treatment. After this, within two weeks, the symptoms gave way.

Conclusions: The syndrome of cannabinoid hyperemesis consists of nausea and vomiting that normally appear at 12 hours after cessation of consumption. These symptoms can last 3 weeks and they improve with the use of frequent hot showers. It has been proposed, as a possible explanation, an increase in the proemetic metabolites of cannabis, as well as a splenic vasodilation, which decreases when applying the hot showers that redistributes the blood flow to the skin and relieves the symptoms. It is important to think of this chart in chronic cannabis users.

Conflict of interest: No

Keywords: cannabis; Liaison Psychiatry; shower; cannabinoid hyperemesis

\section{EPV0364}

\section{Korsakoff síndrome. Clinical features and treatment. Case report}

C. Carrajo Garcia ${ }^{1 \star}$, M. Rodriguez De Lorenzo ${ }^{1}$, E. Alonso Sánchez $^{2}$, C. Loeck De Lapuerta ${ }^{1}$ and P. Del Sol Calderón ${ }^{3}$

${ }^{1}$ HOSPITAL UNIVERSITARIO RAMON Y CAJAL, Psychiatry, MADRID, Spain; ${ }^{2}$ HOSPITAL UNIVERSITARIO RAMON Y CAJAL, Psychiatry, Madrid, Spain and ${ }^{3}$ HOSPITAL UNIVERSITARIO PUERTA DE HIERRO MAJADAHONDA, Psychiatry, MADRID, Spain

${ }^{*}$ Corresponding author.

Introduction: A 58-year-old male, who is admitted to Internal Medicine due to behavioral disturbance after being found on public roads, with bruises and temporo-spatial disorientation. In medical history, multiple visits to the Emergency Department are highlighted due to alcohol poisoning.

Objectives: The aim of this case is to show the importance of cognitive screening in alcohol abuse

Methods: During the anamnesis performed by Link Psychiatry, the patient verbalizes religious-spiritual content, with a low degree of systematization, in addition to referring to recurring phenomena of false recognition of family members. The affection is incongruous and the degree of consistency and coherence shown in the discourse is low, modifying throughout the days the content and thematic of the ideas presented, giving contradictory information regarding the same subject depending on the moment. In the last year the family reports apathetic / abustic functioning as well as deterioration for self-care. Cranial CT, analytical with serologies and toxic screening without findings are performed. A neurological examination 
highlights an emerging difficulty in executive tests and immediate memory.

Results: During hospitalization, the patient received treatment with thiamine at a dose of $300 \mathrm{mg} /$ day and risperidone $1 \mathrm{mg} /$ day. The diagnosis at discharge was of amnesic-confabulatory syndrome, compatible with Korsakoff syndrome (F10.6) and harmful alcohol consumption (F10.1)

Conclusions: Korsakoff Syndrome is a residual and irreversible syndrome that occurs when Wernicke's Encephalopathy has not been treated immediately and properly. The interventions will be aimed at improving the remaining skills, as well as suppressing symptoms that interfere with normal functioning.

\section{Conflict of interest: No}

Keywords: Alcohol; Cognitive evaluation; thiamine; amnesicconfabulatory syndrome

\section{EPV0365}

Tacrolimus associated with reversible posterior encephalopathy in liver transplant recipient

C. Carrajo Garcia ${ }^{1 \star}$, E. Alonso Sánchez ${ }^{2}$, C. Loeck De Lapuerta ${ }^{1}$, M. Rodriguez De Lorenzo ${ }^{1}$ and P. Del Sol Calderón ${ }^{3}$

${ }^{1}$ HOSPITAL UNIVERSITARIO RAMON Y CAJAL, Psychiatry, MADRID, Spain; ${ }^{2}$ HOSPITAL UNIVERSITARIO RAMON Y CAJAL, Psychiatry, Madrid, Spain and ${ }^{3}$ HOSPITAL UNIVERSITARIO PUERTA DE HIERRO MAJADAHONDA, Psychiatry, MADRID, Spain

${ }^{*}$ Corresponding author.

Introduction: 62-year-old woman, admitted to the Gastroenterology Department for liver transplantation secondary to cryptogenic cirrhosis. During admission she presents episodes of tempospace disorientation and psychomotor agitation, for which assessment is requested by Psychiatry and Neurology.

Objectives: The objective is to show part of the post-transplant neurological clinic and how to manage it

Methods: Upon examination, the patient is conscious and alert but disoriented in time and space. It presents a globally coherent and well-articulated discourse plagued with spontaneous confabulations, congruent with behavior and consistent in time that limits the grandiosity and overestimation of one's abilities. Emotional lability and irritability at times. No insight. Judgment of altered reality. In the cognitive assessment carried out, short-term memory deficits and intrusions are observed. During admission, EEG was performed, lumbar puncture, analytical with serologies and toxic detection that were anodyne. In cranial NMR, diffuse parenchymal atrophy, periventricular leukoaraiosis and hyperintensity of the signal in both pale ones are observed, in relation to cation deposition compatible with suspected encephalopathy.

Results: Given the suspicion of encephalopathic symptoms in relation to post-transplant drug treatment, the change of tacrolimus to everolimus is decided. The patient presents an ad integrum resolution of the picture

Conclusions: Reversible posterior encephalopathy syndrome can occur in transplanted patients of solid organs associated with calcineurin inhibitors. It should be suspected when neurological symptoms associated with characteristic lesions appear on CMR that revert after decreasing the dose of tacrolimus or discontinuing it. From a timely diagnosis and suspension of the calcineurin inhibitor dependent on the resolution of the condition without neurological sequelae.

\section{Conflict of interest: No}

Keywords: solid organ transplant; calcineurin inhibitors; confabulations; tempospatial disorientation

\section{EPV0368}

Psychotic symptoms in parkinson disease. About a case

A. Rodríguez-Quiroga ${ }^{1 \star}$, P. Nava $^{2}$, A.M. Matas Ochoa ${ }^{1}$, R. Martinez De Velasco ${ }^{2}$, C. Banzo ${ }^{2}$ and F. Mora ${ }^{2}$

${ }^{1}$ Hospital Universitario Infanta Leonor, Psychiatry, Madrid, Spain and ${ }^{2}$ Hospital Infanta Leonor, Psychiatry, Madrid, Spain

${ }^{*}$ Corresponding author.

Introduction: Psychosis is a frequent complication of Parkinson disease. It is mainly characterized by visual hallucinations and delusions. Hallucinations are the most common manifestation and affect up to $40 \%$ of patients, particularly those in an advanced stage of the disease.

Objectives: To describe the relationship between Parkinson disease and psychosis.

Methods: We present the case of a 75-year-old patient diagnosed with Parkinson disease (stage II) who began with visual hallucinations and then developped delusions of harm.

Results: Complementary tests (cranial CT, blood test, ECG) were normal. Ophthalmic pathology was ruled out. Exploration: Hypomimia, monotonous voice. Asymmetrical resting hands tremor, predominantly right, bilateral mild postural tremor. Bradykinesia Right, mild left spontaneous stiffness. Spastic paraparesis Paretoespastic march with support. Conscious and globally oriented. Fluent and spontaneous language, with coherent and structured discourse. Low mood, preserved emotional resonance. Denies autolytic planning. Delusional ideation of harm. Visual and auditory hallucinations. Chronobiological rhythms preserved.

Conclusions: The adverse effects of dopamine agonists are probably the most important cause of psychosis in these patients. The differential diagnosis would include a late onset psychosis, Charles Bonnet syndrome and a secondary psychosis. Oral treatment was started with $100 \mathrm{mg}$ / day of quetiapine in three doses, with good tolerance and effectiveness on the previously described symptomatology. The recommendations for the management of psychotic symptoms are: 1) Discard intercurrent process. 2) Avoid anticholinergic medication. 3) Reduce or suspend antiparkinsonian medications. 4) Antipsychotic treatment.

\section{Conflict of interest: No}

Keywords: Parkinson disease; psychosis; visual hallucinations; dopamine agonists

\section{EPV0369}

\section{Cardiac rehabilitation. A program that saves lives}

A. Rodríguez-Quiroga ${ }^{1 *}$, P. $\mathrm{Nava}^{2}$, A.M. Matas Ochoa ${ }^{1}$, R. Martinez De Velasco ${ }^{2}$, C. Banzo ${ }^{2}$, M. Villacañas ${ }^{1}$ and F. Mora ${ }^{2}$

${ }^{1}$ Hospital Universitario Infanta Leonor, Psychiatry, Madrid, Spain and ${ }^{2}$ Hospital Infanta Leonor, Psychiatry, Madrid, Spain ${ }^{\star}$ Corresponding author.

Introduction: There is a significant relationship bewtween ischemic heart disease (IHD) and depression or anxiety, with $20 \%$ of patients having a depressive disorder after acute myocardial infarction (AMI). The first symptoms of depression appear between 
48 and 72 hours after AMI and in most patients disappear within a week. The incidence of depression and anxiety symptoms is important because of the potential impact of these variables on subsequent morbidity and mortality of these patients.

Objectives: To describe the cardiac rehabilitation program designed in our hospital.

Methods: We describe an intensive, multimodal, 8-week cardiac rehabilitation treatment program, for patients who have had a cardiac event.

Results: The program is comprised by different professionals: cardiologist, nurse, physiotherapist, psychologist and psychiatrist, who work in a coordinated manner to ensure the optimal and comprehensive treatment of all patients who are part of the program. So far, up to four patients, out of sixty, have required specific treatment with psychotropic drugs for depressive and anxious symptoms. Both at baseline and after 8 weeks of treatment, the Hamilton Depression Rating Scale (HAM-D) and the Montgomery Asberg Depression Rating Scale (MADRS) were applied to patients who showed depressive symptoms, with significant improvement in the scores of both scales at the end of the treatment program.

Conclusions: Anxiety and depression should be closely monitored in all patients with cardiovascular disease using simple screening methods, as they are important predictors of the outcome in these kind of patients.

Conflict of interest: No

Keywords: Dépression; ischemic heart disease; adjustment disorder; acute myocardial infarction

\section{EPV0372}

\section{Sexual improvement in hepatitis $\mathrm{C}$ infected patients} cured after direct acting antivirals: one-year follow-up.

M. Cavero ${ }^{1 \star}$, R. Navines ${ }^{1}$, S. Rodriguez-Tajes ${ }^{2}$, S. Lens ${ }^{2}$, C. Bartres ${ }^{2}$, L. Nácar ${ }^{1}$, A. Pla ${ }^{2}$ A. Miralpeix ${ }^{2}$, M. Gonzalez ${ }^{3}$, J. Sarquella ${ }^{3}$, L. Sastre ${ }^{2}$, R. Martin-Santos ${ }^{1}$, X. Forns ${ }^{2}$ and Z. Mariño ${ }^{2}$

${ }^{1}$ Hospital Clinic, UB, IDIBAPS, CIBERSAM, Psychiatry And Psychology, Barcelona, Spain; ${ }^{2}$ Hospital Clinic, UB, IDIBAPS, CIBERehd, Liver Unit, Barcelona, Spain and ${ }^{3}$ Fundació Puigvert, Andrology Department, Barcelona, Spain

${ }^{\star}$ Corresponding author.

Introduction: Hepatitis $\mathrm{C}(\mathrm{HCV})$ infected patients have a high rate of sexual dysfuntion associated with age, depression and subjective limitations due to HCV (Mariño et al., 2017). Current antiviral therapies with direct acting antivirals (DAA) improve quality of life, although especific analysis of sexuality has been limited.

Objectives: To assess the impact of substained virological response after DAA on sexual quality of life of HCV patients.

Methods: Prospective inclusion of patients receiving DAA (AprilDecember, 2018) recording Clinical and socio-demographic variables. Patients were assessed at baseline and 48-weeks after DAA (FU48) using the Spanish validated versions of Changes in Sexual Functioning Questionnaire (CSFQ-14/CSFQ-VAS) (GarcíaPortillo et al., 2011), and Patient Health Questionnaire (PHQ-9) (Navines et al., 2012). They were asked about fear for HCV sexual transmission and HCV-related limitations in their sexual lifes. Results were expressed in median/IQR(25-75) and n(\%). Paired samples were analyzed by McNemar and T-tests. Logistic regression analysis was applied.

Results: One-hundred-eighty-six (83\%) accepted to participate, $55 \%$ men, median age of 55(49-61) years, $20 \%$ have a current major depression, 64\% have sexual dysfunción (62\% males). At FU48 $(\mathrm{n}=151,81 \%)$ it was observed an improvement in Sexual dysfunction $(p<0.035)$ mainly in youngers, CSFQ-14 score $(p<0.002)$, CSFQ-VAS $(\mathrm{p}<0.001)$, Fear to HVC transmission $(\mathrm{p}=0.03)$. No differences was observed in depression prevalence $(p=0.85)$ nor in sexual limitation atributable to HCV $(\mathrm{p}=0.4)$. Age $(\mathrm{p}<0.001)$ and PHQ-9>10 $(\mathrm{p}<0.001)$ were associated to sexual dysfunction persistence at FU48.

Conclusions: The results support to explore sexual dysfunction in patients with hepatitis $\mathrm{C}$ before and after DAA treatment, due the highly prevalence and their improvement after cure.

Conflict of interest: No

Keywords: antivirals; Sexual improvement; hepatitis C

\section{EPV0375}

Mutism culminating in a diagnosis of creutzfeldt-jakob disease - a case report

\section{E. Machado*, L. Fonseca, J. Fonseca, R. Rodrigues and S. Simões}

Hospital da Senhora da Oliveira, Psychiatry And Mental Health,

Guimarães, Portugal

${ }^{\star}$ Corresponding author.

Introduction: Mutism, defined as an inability or unwillingness to speak resulting in an absence or marked paucity of verbal output, is a common clinical symptom seen in psychiatry as well as neurology illness. It rarely presents as an isolated disability and often occurs in association with other disturbances of behavior, thought, affect or state of consciousness.

Objectives: To report a case of Creutzfeldt-Jakob Disease (CJD) and to discuss the Clinical and diagnostic considerations in the workup for mutism.

Methods: Assessment of the patient and interview with family members.

Results: A 77-year-old woman presented with a 6-month history of rapidly progressive cognitive impairment and gait decline. She had a history of mixed anxiety and depressive disorder and a convexity meningioma in the right parietal lobe. She was admitted to the medicine ward because of temporo-spatial desorientation of sudden onset, being first treated for a urinary tract infection. Throughout her hospitalisation the most prominent symptoms were mutism and food refusal. In this context, an observation by neurology and psychiatry was requested. Following an extensive battery of tests, the established diagnosis was probable CJD. She passed away 12 weeks after his initial presentation to hospital, and an autopsy confirmed a diagnosis of sporadic CJD.

Conclusions: Our case highlights the importance of a high index of suspicion for non-psychiatric causes for mutism before atributing a definite psychiatric ethiology, as this will decide the management and prognosis of the patient.

Conflict of interest: No

Keywords: mutism; creutzfeldtjakobdisease

\section{EPV0378}

Complications of alcohol use disorder: a view from the liaison psychiatry service

P. Muñoz-Calero ${ }^{1 *}$, A. Muñoz Domenjó ${ }^{2}$, N. Rodríguez Criado ${ }^{3}$ and E. Ramos García ${ }^{2}$ 
${ }^{1}$ Hospital Universitario de Móstoles, Psychiatry, MADRID, Spain;

${ }^{2}$ Hospital Universitario de Móstoles, Psiquiatría, Móstoles, Madrid, Spain and ${ }^{3}$ Hospital Infantil Universitario Niño Jesús, Child And Adolescent Psychiatry And Psychology Department, Madrid, Spain ${ }^{\star}$ Corresponding author.

Introduction: Alcohol use disorder is one of the most prevalent disorders that we treat in the Liaison Psychiatry Service in our hospital. The complications related with this disorder are many and can affect nearly every system in our body.

Objectives: Our objectives in this poster is to describe the main complications of alcohol use disorder so we can detect and treat them in their early presentation.

Methods: Systematic review of the scientific literature related with complications of alcohol use disorder. We will also use some of our cases to describe these complications.

Results: Alcohol use disorder and its complications is the third cause of referral in our Liaison Psychiatry Service.

Conclusions: Complications related with alcohol use disorder are one of the main causes of global burden.To detect and treat these complications in their early presentations may change the prognosis of alcohol use disorder.

Conflict of interest: No

Keywords: complications; Alcohol; Liaison Psychiatry

\section{Cultural psychiatry}

\section{EPV0392}

\section{Culture and personality disorders-a case series.}

I. Cuevas ${ }^{\star}$, L. Fontecha Banegas, I. Moreno Alonso and M. Blanco Prieto

Hospital Universitario Principe de Asturias, Psiquiatria, Alcala de Henares, Spain

${ }^{\star}$ Corresponding author.

Introduction: Personality disorders comprise a set of diagnosis characterized by inflexible, pervasive and enduring patterns of cognition, affect, behavioural and social interaction. The status of research on the personality among different cultures implies the universality of traits and disorders, as well as, their measures across cultures.

Objectives: To study the prevalence of personality disorders in foreigner patients.

Methods: In this retrospective case series, clinical data was collected from 40 patients who were hospitalized at the short-stay inpatient psychiatric service of the Príncipe de Asturias University Hospital during 2018.

Results: Nineteen (47.5\%) patients were European, ten (25\%) were from South America, nine (22.5\%) were African and two (5\%) were Asian. Eight patients were diagnosed of diverse personality disorders. Seven (87.5\%) of them were European, and only one (12.5\%) was from South America.

Conclusions: This case series suggests various directions for future research. The fact that patients diagnosed with personality disorders were mainly European could indicate diverse conclusions. It would question the universality of personality disorders out of a Euro-american frame of reference. It would also point out the difficulty of diagnosing personality disorders, taking into consideration language, awareness of cultural values, traditions, interactional patterns, and social norms. More studies of traits and personality are needed, taking into account the culture and the society in which patients have grown and in which they currently live.

Conflict of interest: No

Keywords: Personality disorders; cross-cultural

\section{Depressive disorders}

\section{EPV0412}

\section{¿Dead or alive? cotard syndrome: a case report.}

M.P. Algarín Moriana ${ }^{1 \star}$, A.H. Guzmán Del Castillo ${ }^{1}$, E.J. López Sánchez $^{2}$, A. Sánchez Gayango ${ }^{2}$ and G. Velarde Pedraza ${ }^{1}$

${ }^{1}$ Hospital Universitario Nuestra Señora Virgen de Valme, Psiquiatría, Sevilla, Spain and ${ }^{2}$ Hospital Nuestra Señora de Valme, Psiquiatría, Sevilla, Spain ${ }^{\star}$ Corresponding author.

Introduction: Electroconvulsive therapy involves the induction of a seizure by applying an electrical stimulus through electrodes, usually on both sides of the scalp. It remains being one of the main treatments for severe and psychopharmacological treatmentresistant mental illnes.

Objectives: This case aims to show the evolution of a severe depressive episode after electroconvulsive therapy.

Methods: Case report.

Results: We present a case of a 75-year-old woman, with no previous history in Mental Health who consults in the emergency department for a psychomotor inhibition and negative for both water and food intakefor a month of evolution. The patient began presenting excessive fear of being alone and of performing activities, showing herself more and more clumsy, and finally becoming completely dependent on the basic activities of daily living. During hospitalization in Psychiatry, the patient repeatedly referred to the conviction of being dead and of not needing to eat or drink because of it. Inhibition episodes alternated with psychomotor restlessness, and olfactory and gustatorysensory perceptual changes appeared during hospitalization. Symptons did not improve after the introduction of antidepressant or antipsychotic treatment, being finally necessary the application of electroconvulsive therapy, presenting the patient clinical improvement progressively until the depressive symptomatology finally disappeared.

Conclusions: Serious depressive episodes, associated or not with psychotic symptoms, are an indication of electroconvulsive therapy. In this case, the rapid onset of the condition, the state of inhibition she presented and the short time of evolution were predictive factors of good evolution, objectifiable from the first sessions.

Conflict of interest: No

Keywords: cotard; syndrome; electroconvulsive; therapy

\section{EPV0452}

Depression and academic learning in children with high intellectual potential (HIP): is there a relationship?

L. Vaivre-Douret 
Faculty of Medicine, University of Paris, Paris Descartes ; INSERM UMR 1178/1018-CESP, Necker-Enfants-Malades University Hospital, AP-HP, France University Institute (IUF), Department of Child Psychiatry, Paris, France

Introduction: The symptoms of the depression in high intellectual potential (HIP) children (IQ > 130) are little studied.

Objectives: The aim of the study is to understand depression versus no depression in a sample of gifted children, with a standardized and normative scale.

Methods: We assessed the depression with the Multiscore Depression Inventory for Children (MDI-C) in a national sample of 361 HIP children (primary school and college) requesting association of gifted children. This assessment is a self-report instrument administrered to 8 to 17 years old, enabling to measure multicomponent symptoms of depression represented in 8 subscales (Anxiety, Self-Esteem, Sad mood, Instrumental helplessness, Social introversion, Low energy, Pessimism, Defiance). All the parents answered to a questionnaire about academic learning of their child. Results: IQ 130-139 versus $>140$ IQ are not significant with depression in primary school $(\mathrm{p}<.43)$ and college $(\mathrm{p}<.26)$. We found $47 \%$ of depression. All the symptoms are associated to the depression score $(\mathrm{p}<.001)$. HIP children with depression have significantly $(\mathrm{p}<.001)$ difficulty at primary school in reading, $23 \%$ (vs) $6 \%$ without depression, respectively $7 \%$ vs $2 \%$ for written expression $(\mathrm{p}<0,003)$ and $7 \%$ vs $2 \%$ for arithmetic $(\mathrm{p}<.003)$. At the college, there is an increase of learning disabilities.

Conclusions: IQ score is not in relationship with depression. However IQ > to 130 may mask school disabilities, so it appears necessary to examine the subtests of IQ which are significantly low, and assess neuropsychological and motor brain functions.

Conflict of interest: No

Keywords: Dépression; Academic learning; High intellectual potential child; IQ

\section{Eating disorders}

\section{EPV0489}

Neuropsychological aspects in eating disorders: the rey complex figure test

B. Mata-Saenz ${ }^{1}$, V. Muñoz-Martinez ${ }^{1}$, I. Diaz-Quero ${ }^{1 \star}$, V. QueroPalomino $^{1}$, G. Belda-Moreno ${ }^{1}$, M. Leon-Velasco ${ }^{1}$, L. BeatoFernandez $^{1}$ and T. Rodriguez-Cano ${ }^{2}$

${ }^{1}$ Hospital General Universitario de Ciudad Real, Psychiatry, Ciudad Real, Spain and ${ }^{2}$ Servicios Centrales SESCAM, Coordinación Regional De Salud Mental, Toledo, Spain

${ }^{*}$ Corresponding author.

Introduction: Eating disorders (ED) present low diagnosis stability based on current diagnostic classifications. That's why, transdiagnosis approach and endophenothype concept take relevance.

Objectives: The aim of the present study was to perform a descriptive analysis of neuropsychological variables, which could be related, considering demographical and psychopathological aspects.

Methods: A descriptive analyse was performed with 81 female patients with ED between 18-50 years old. Socio-demographical, Clinical and neuropsychological variables were collected.

Results: From the 81 initially included patients, 7 did not meet some of the inclusion criteria and 24 were lost during the follow-up.
The sample was reduced to 51 patients. The Rey Complex Figure Test (ROCFT) analysis yielded the following results: Copy time: mean (M) 2.94 (standar deviation, SD 1.12), Copy: M 34.28 (SD 2.76), Memory: M 19.47 (SD 6.02), Order: M 2.14 (SD 0.73), Style: M 1.54 (SD 0.32), Coherence: M 1.42 (SD 0.34).

Conclusions: Time of ROCTF copy was found in the 50th percentile. The accuracy and richness of the copy were on average in the 80th percentile, due to the perfectionism patients' tendency. However, in the memory figure, the mean was in the 40th percentile with greater variability. Probably, because of fact that $27 \%$ of the patients developed it with an unusual pattern in order to their age, similar found in others studies that highlighted deficiencies in central coherence. The global analysis of the sample, only show promising results regarding executive functions, especially central coherence, congruent with those expected and found in previous studies.

Conflict of interest: No

Keywords: Neuropsychology; eating disorders; The Rey Complex Figure Test; Executive Function

\section{EPV0490}

\section{Assessment of autistic traits in adolescents with eating disorders.}

V. Muñoz-Martínez, B. Mata-Saenz, T. Rodriguez-Cano, I. DiazQuero $^{*}$, V. Quero-Palomino and L. Beato-Fernandez

Hospital General Universitario de Ciudad Real, Psychiatry, Ciudad

Real, Spain

${ }^{*}$ Corresponding author.

Introduction: The overlap between autism spectrum disorders (ASD) and eating disorders (ED) has been studied for years, finding similarities in certain cognitive characteristics.

Objectives: The aim of this study is to analyze the available literature about the relationship between autism spectrum symptoms with different pathologies which are include in eating disorders.

Methods: Information was collected from different databases (Pubmed, Cochrane, Tripdatabase, UpToDate) between 2013 and 2018. Descriptors as "eating disorders", "autism", "treatment", "psychotherapy", "anorexia nervosa", are used. "Bulimia nervosa”, "food avoidant / restriction intake disorder", "binge eating disorder", "obesity" and "adolescence" and the same ones in Spanish are used

Results: The published studies, referred throughout the text, mainly refer to anorexia nervosa (AN) with less research in others eating disorders such as obesity, bulimia, and binge eating disorder. Conclusions: However, despite of the fact that the relationship with the AN is especially mentioned, there are cognitive characteristics that are shared between the ED and the ASD that must be taken into account due to their implications, not only in treatment, also in the prognosis.

\section{Conflict of interest: No}

Keywords: Autism; Eating Disorders; Adolescents; Outcome; Treatment.

\section{EPV0506}

\section{How dissociation influences in eating disorders} prognosis

V. Muñoz-Martínez, B. Mata-Saenz, T. Rodriguez-Cano, V. QueroPalomino* and I. Diaz-Quero 
Hospital General Universitario de Ciudad Real, Psychiatry, Ciudad Real, Spain

${ }^{\star}$ Corresponding author.

Introduction: Eating disorders (ED) are illnesses with high sanitary repercussions because of their frequency and prognosis. Different investigations were conducted to determine which factors can influence the prognosis. Disassociation is one of them. Disassociation is the separation of the contents of the mind, which normally appear to be integrated into the conscious.

Objectives: The hypothesis proposes that high disassociation levels can interfere in the therapeutical response and, contrarily at lower levels, disassociation can show improvement in these patients.

Methods: 119 patients were recruited from the Eating Disorders outpatient unit. Different questionnaires were administered. These questionnaires analysed general and eating psychopathology, the "Dissociative Experiences Scale" -DES- and the "Attitudes Toward Change in Eating Disorders" -ACTA-. After a year, the questionnaires were re-administered analysing the relationship between "DES" scores with other psychopathological scores.

Results: The dissociative levels which patients presented at the beginning influenced general and eating psychopathology. Moreover, we determined that final dissociative levels had influence on the attitude toward change and this one had influence in both general and eating psychopathology, except in "anxiety trait" which was associated with disassociative levels despite the "ACTA"scores.

Conclusions: Dissociation has influence on the prognosis of patients with an Eating Disorder, independent of the diagnosis category, because of the relationship with changes in eating and general psychopathology. Attitude towards change mediate this effect; lower levels of disassociation were associated with a proactive attitude in therapy and in the maintenance of change.

Conflict of interest: No

Keywords: Eating Disorders; Dissociative Experiences. Attitude Towards Change.

\section{EPV0507}

Neuropsychological impairment associated with depression in a sample of patients with eating disorders

B. Mata-Saenz ${ }^{1}$, V. Muñoz-Martinez ${ }^{1}$, V. Quero-Palomino ${ }^{1 \star}$, I. Diaz-Quero ${ }^{1}$, M. Leon-Velasco ${ }^{1}$, G. Belda-Moreno ${ }^{1}$, T. Rodriguez-Cano ${ }^{2}$ and L. Beato-Fernandez ${ }^{1}$

${ }^{1}$ Hospital General Universitario de Ciudad Real, Psychiatry, Ciudad Real, Spain and ${ }^{2}$ Servicios Centrales SESCAM, Coordinación Regional De Salud Mental, Toledo, Spain

${ }^{\star}$ Corresponding author.

Introduction: Neuropsychological disturbances are shown in studies about eating disorders (ED). On the other hand, attention impairment is related with cronic depression and anxiety, with poor improvement with pharmachological treatment. Important comorbility between ED and other psychiatric diseases is described, specially with depression and anxiety.

Objectives: The aim of this study was to evaluate the influence of depression in neuropsychological variables in eating disordered patients.
Methods: A descriptive analyse was performed with 81 female patients with ED between 18-50 years old. Socio-demographical, Clinical and neuropsychological variables were collected. Depression was measured by Beck Depression Inventory (BDI).

Results: A relationship between depression (dicotomic variable) and Symbol Digit Modalities Test (SDMT) was found $(\mathrm{p}<0.05, \mathrm{Z}$ -1.69 , average range without depression 32.64 vs average range with depression 23.49). Attending to cuantitative result of BDI, relationships with Letter and Number Sequencing (total score: $\mathrm{p}<0.05$, rho -0.29; SpanLN: $\mathrm{p}<0.05$, rho -0.28$)$ and SDMT $(\mathrm{p}<0.05, \rho$ Pearson -0.28 ) were found.

Conclusions: In ED impairment of attention and working memory have been related to executive function or stimuli distortion. In our sample the scores are similar to normal population but they're related to severity of depression.

\section{Conflict of interest: No}

Keywords: Dépression; Neuropsychology; Cognitive impairment; Eating Disorders

\section{EPV0511}

Is family therapy really helping our patients with eating disorders? A 6-year retrospective study

B. Martins Ramos ${ }^{\star}$, F. Coutinho, A. Delgado, D. Barbosa, E. Osório and I. Brandão

São João Hospital and University Center, Psychiatry And Mental Health Department, Porto, Portugal

${ }^{*}$ Corresponding author.

Introduction: Ongoing research has highlighted the efficacy of family therapy (FT) in the treatment of adolescent anorexia nervosa (AN) and bulimia nervosa (BN).

Objectives: In this study we aim to characterize patients treated in a specialized Eating Disorder Unit in São João University and Hospital Center over a period of 6 years with a treatment plan including FT. We aim to present the clinical results from engaged patients, comparing the mean Body Mass Index (BMI) and the number of inpatients admissions before and after they started FT and try to stablish a correlation between those.

Methods: Retrospective analysis of clinical processes of patients with ED enrolled in family therapy between 2013 and 2018. Statystical analysis using IBM SPSS Statistics 26.

Results: 49 patients were monitored during this period and had FT as a part of treatment plan (45 female and 4 male). Regarding diagnosis, 42 had $\mathrm{AN}$ and 7 had BN. After this period of treatment the number of inpatient admissions significantly decreased for all diagnosis patients. Patients with AN had an increase in the mean BMI after concluding FT, also statistically significant.

Conclusions: This program provides a stable and prolonged treatment aiming to be flexible and adapted to the patient needs. Despite limitations considering the small population and that FT was not the only intervention administered to these patients, our data suggests that FT was a useful intervention in the sample.

\section{Conflict of interest: No}

Keywords: eating disorder; family therapy; Anorexia nervosa; Bulimia nervosa 


\section{Emergency psychiatry}

\section{EPV0547}

\section{Corticosteroid-induced mania in the emergency room:} a case report.

A. Bermejo Pastor ${ }^{1}$, R. Galerón Guzmán ${ }^{1}$, E. Herrero Pellón ${ }^{1}$, M. Huete Naval ${ }^{1}$, P. Albarracín Marcos ${ }^{1}$ and Í. Alberdi Páramo ${ }^{2}$

${ }^{1}$ Hospital Clínico San Carlos., Institute of Psychiatry And Mental Health., Madrid, Spain and ${ }^{2}$ Hospital Universitario de la Princesa, Psychiatry Department, Madrid, Spain

${ }^{*}$ Corresponding author.

Introduction: Corticosteroids are commonly used for several conditions. Among their various adverse effects, psychiatric conditions have been described. We present a 53-year-old woman on treatment with $60 \mathrm{mg}$ prednisone for eosinophilic pneumonia, who enters to the emergency room. Interviewing the patient and her family, they describe a clinical picture, in the last 4 days, of euphoria, insomnia, irritability, increase of activity, restless and transient verbalization of delusional ideas. The patient has no psychiatric personal history, although a significant family history of depression, mania and psychosis. Olanzapine $7,5 \mathrm{mg}$ was added and the symptoms disappeared gradually in 3 weeks.

Objectives: To review the epidemiology and clinical features of the corticoid induced mania, as well as its clinical management in the emergencies room.

Methods: Review of the literature on manic episodes induced by corticosteroids applying the information to this case.

Results: Among patients receiving doses greater than $40 \mathrm{mg}$ prednisone, $20 \%$ will suffer psychiatric disorders severe enough to require medication. Manic symptoms are the most common manifestation of these disorders and account for about $40 \%$ of them. The onset of these symptoms is commonly after 10 days of sustained corticosteroid treatment. Reducing doses or cessation, if possible, should be the first line for treatment. Antipsychotic treatment, such as olanzapine or quetiapine is supported by literature.

Conclusions: Corticosteroid induced mania is the most common severe neuropsychiatric disorder caused by corticosteroids. -Doses greater than $40 \mathrm{mg}$ of prednisone and longer than 10 days increase the risk. -Cessation of corticosteroids and antipsychotics are effective treatments.

Conflict of interest: No

Keywords: mania; corticosteroids; Bipolar; Emergency

\section{EPV0549}

\section{The lunatic association between full moon and psychiatric emergency care visits}

D. Hernández-Calle ${ }^{1}$, A. Garcia Cardenal ${ }^{1}$, G. Kollias ${ }^{1}$, A. Suárez Lorenzo $^{1}$ and M.F. Bravo-Ortiz ${ }^{2}$

${ }^{1}$ Hospital Universitario La Paz, Psychiatry, Madrid, Spain and ${ }^{2} \mathrm{La}$ Paz University Hospital Research Health Institute (IdiPAZ), Psychiatry, Clinical Psychology And Mental Health, Madrid, Spain

${ }^{\star}$ Corresponding author.

Introduction: Even though that the etiopathogenic hypothesis that associates cerebral changes produced by the Moons gravity and mental disorders has been disproved, when the night shift has been thought it is often heard "It surely was full moon!".
Objectives: To study de association of full moon and number of Psychiatric Emergency Care visits

Methods: We obtain the daily number of Psychiatric Emergency Care visits from September 2018 to July 2019 and compared the median of full moon days and not-full moon days using the Wilcoxon rank-sum test.

Results: The median of the number of Psychiatric Emergency Care visits of full moon days was 7 (95\%CI 2.65- 9.35) and without full moon was 7 (95\%CI 6.75-7.25). The difference between both medians was not statistically significant (WRS p 0.51).

Conclusions: There are not more Psychiatric Emergency Care visits in full moon days.

\section{Conflict of interest: No}

\section{EPV0566}

Flumazenil response in hepatic encephalopathy: a case report.

R. Fernández ${ }^{1 \star}$, C. Harkous $^{1}$, R. Blanco ${ }^{2}$, A. Rodríguez ${ }^{3}$, M. Vizcaíno Da Silva ${ }^{3}$ and O. Mendez Gonzalez

${ }^{1} \mathrm{HM}$ CINAC, Servicio De Psiquiatría Y Psicología Clínica Hm Hospitales, Móstoles, Spain; ${ }^{2}$ HOSPITAL UNIVERSITARIO PUERTA DE HIERRO MAJADAHONDA, Psychiatry, MADRID, Spain and ${ }^{3}$ Centro de Salud San Carlos, Psiquiatría, San Lorenzo del Escorial, Spain

${ }^{*}$ Corresponding author.

Introduction: A 47-year-old woman requires urgent transfer to the hospital due to a low level of consciousness. When she arrives, flumazenil is given with good response, the toxins are positive for benzodiazepines, however, the patient denies having performed a medication overdose and comments on taking lorazepam to sleep. It was not possible to reduce the infusion of flumazenil due to persistence of symptoms, we found a severe alteration of liver transaminases and an increase in alkaline phosphatase; given the diagnostic suspicion of hepatic encephalopathy, treatment with lactulose is initiated with a good response.

Objectives: The case of a woman with hepatic encephalopathy and response to flumazenil is described.

Methods: We made a literature review.

Results: Exposed in conclusions.

Conclusions: Hepatic encephalopathy is a neuropsychiatric condition that can begin as drowsiness and alteration of the normal sleep pattern, with a progressive alteration of consciousness and generalized motor dysfunction. Ammonium is the main substance involved in the pathophysiology, but there are other theories that refer to GABA produced at the intestinal tract can act as a neuroinhibitor, relating to the identification of endogenous benzodiazepines (Idrovo, 2003), or modulators of the non-benzodiazepine GABA-A complex (Ahboucha, 2005). Numerous studies have proposed the effectiveness of flumazenil as a treatment of hepatic encephalopathy, however we must be cautious: in a systemic review published in the Cochrane Database (Goh, 2017) there is low quality evidence suggesting a short-term beneficial effect of flumazenil on hepatic encephalopathy in people with cirrhosis, but no evidence of an effect on all-cause mortality, recommending longterm studies.

Conflict of interest: No

Keywords: flumazenil; hepatic encephalopathy; benzodiacepines; GABA 


\section{Epidemiology and social psychiatry}

\section{EPV0577}

Back pain treatment for patients with mental disorders

M. Lachina ${ }^{1 \star}$, P. Ljungdalh ${ }^{2}$, O. Garvik ${ }^{1}$, E. Stenager ${ }^{3}$, B. SchiøttzChristensen $^{4}$ and B. Nørgård ${ }^{1}$

${ }^{1}$ Center for Clinical Epidemiology, Odense University Hospital and University of Southern Denmark, Department of Clinical Epidemiology, Odense C, Denmark; ${ }^{2}$ The Spine Centre of Southern Denmark, Medical Research Department, Middelfart, Denmark;

${ }^{3}$ Psychiatric Research Unit, Department of Regional Health Research, University Of Southern Denmark, Åbenrå, Denmark and ${ }^{4}$ The Spine Centre of Southern Denmark and Institute of Regional Health Science, Medical Research Department, Middelfart, Denmark

${ }^{\star}$ Corresponding author.

Introduction: Prior studies have shown that back pain and mental disorder are common, and sometimes related, conditions in the general populations. A better understanding of the relationship between mental disorders, back pain conditions, and the offered therapies is essential for clinicians.

Objectives: To examine, association between pre-existing mental disorder and the chance of having proper back pain.

Methods: This study was a retrospective cohort study utilizing Danish registries. Using the Danish Registers we identified patients with back pain diagnosis and who of those had a pre-existing mental disorder. For the study purpose we defined two outcome measurements related to hospital treatment: surgery and establishment of a rehabilitation plan and one related to treatment outside of the hospital: physiotherapy or chiropractic treatment.

Results: Over study time period 2011-2015, we identified 39,380 patients with a back pain diagnosis, 2,097 of those had a preexisting psychiatric disorder. We found that patients with a pre-existing psychiatric disorder do not have less chance of receiving a surgical back pain treatment or establishment of a rehabilitation plan. The chance of receiving a physiotherapy or chiropractic treatment for patients with pre-existing psychiatric disorder even after adjusting for age, sex, socio-economic status, education, social group, and somatic co-morbidity was on the other hand significantly lower with an estimated HR at 0.73 (95\%CI $(0.62 ; 0.88)$ ).

Conclusions: Our study shows that patients with a pre-existing psychiatric disorder need special attention when it comes to back pain treatment outside the hospital setting.

Conflict of interest: No

Keywords: back pain; pre-existing mental disorders

\section{EPV0583}

\section{Cyberbullies or cybervictims: does gender and age} matter?

L. Sahli ${ }^{1}$, S. Bourgou ${ }^{1}$, W. Askri ${ }^{1}$, S. Haj Amor ${ }^{2}$, R. Fakhfakh ${ }^{2}$ and A. Belhadj ${ }^{1}$

${ }^{1}$ Mongi Slim Hospital, Child Psychiatry Department, Tunis, Tunisie, Tunisia and ${ }^{2}$ Medical Center, Birsa, Tunis, Tunisia, ${ }^{3}$ National Public Health Institute, Preventive Medecine Department, Ariana, Tunisia ${ }^{\star}$ Corresponding author.
Introduction: Cyberbullying is the act of using unjustified aggression to harm or harass via digital devices. This widespread phenomenon has serious consequences for individuals and societies. Objectives: The aim of our study was to study the nature of cyberbullying among tunisian and the difference by gender.

Methods: Pupils enrolled in middle and high schools in Tunis, (Tunisia) completed the tunisian adaptation of the Second Revised Cyberbullying Inventory (RCBI-II). This study was developed by the Child Psychiatry Department of Mongi Slim Hospital, (Tunis, Tunisia). Results: A sample of 935 adolescents aged between 13 and 19 years old (average age: 14.2 years old) completed the questionnaire. The sex ratio was 0,85 . The results reveal that $17,9 \%$. were pure cybervictims, $8,4 \%$. pure cyberbullies, $17,1 \%$ cyberbully-victims. There were significant gender differences in cyberbullying. Boys were significantly more likely to carry out cyberbullying than girls $\left(\mathrm{p}=0,011 ; \chi^{2}=6,427 ; \mathrm{dl}=1\right)$; whereas girls were more likely to be cybervictims $(\mathrm{p}=0,014 ; \chi 2=5,981 ; \mathrm{dl}=1)$. Cybervictims were significately aged under the age of 16 years $(p=0.000)$. no significtive difference was find for the cyberbullies statut.

Conclusions: Our findings show that cyberbullying is considerably prevalent among tunisian adolescents. Increased awareness and interventions are now required within schools to tackle this escalating phenomenon.

Conflict of interest: No
Keywords: adolescent; aggression

\section{EPV0589}

\section{Academic burnout in third year students of medicine.}

V. Quero-Palomino*, R. Torralba-Viorreta, A. Gomez-Peinado, B. Mata-Saenz, V. Muñoz-Martínez and I. Diaz-Quero

Hospital General Universitario de Ciudad Real, Psychiatry, Ciudad Real, Spain

${ }^{\star}$ Corresponding author.

Introduction: The stress experienced by university students in the academic context constitutes a topic of increasing interest in recent years, and especially among students of Health Sciences, due to its implications on student health, learning and academic performance. Objectives: To analyze the presence and intensity of academic bournout ande the main academic stressors involved.

Methods: A retrospective, descriptive study was performed. The study population included 80 students of the third course of Medicine of the UCLM in Ciudad Real. Two self-administered questionnaires were used: the SISCO inventory of academic stress and the one-dimensional scale of student burnout.

Results: All of them reported having suffered academic burnout, with a predominance of medium-high intensity. Women have presented higher levels compared to men. The main demands of the academic environment most frequently valued as stressors have been: the overload of homework and work, the limited time to do homework and teacher evaluations.

Conclusions: The growing concern of universities to offer quality education should consider all the variables involved in the teachinglearning process. In this sense, it is important to identify the role of stress factors during the student's training and their relationship with their mental health and academic performance, as well as the moderating role of other variables (age, self-concept, coping strategies ...). Early detection will help to contemplate the need to review 
the teaching methodology, providing contextualized attention and oriented to the improvement of the quality of life of the student, the training institution and the health institutions where their future clinical practice will be developed.

Conflict of interest: No

Keywords: academic burnout; academic stressors; Medicine students; mental health

\section{EPV0590}

\section{Academic stress and coping strategies in medical university students}

V. Quero-Palomino*, A. Gomez-Peinado, R. Torralba-Viorreta, B. Mata-Saenz, V. Muñoz-Martínez and I. Diaz-Quero

Hospital General Universitario de Ciudad Real, Psychiatry, Ciudad Real, Spain

${ }^{*}$ Corresponding author.

Introduction: Academic stress is a widespread phenomenon among students worldwide, being especially high among the university population and even more so in the area of Health Sciences. Studies in this academic context have focused on assessing the main stressors and coping strategies used. The concept of stress coping strategies has received increasing attention from psychologists and health professionals. It is one of the most popular concepts in current psychological research. Two coping styles predominate: focused on solving the problem (active), or focusing on emotion control (passive). The search for social support has mixed components and can be considered as an intermediate coping modality.

Objectives: To analyze the coping styles most frequently used by medical university students.

Methods: A retrospective, descriptive study was performed. The study population included 80 medicine students of the UCLM. The Academic Stress Coping Scale (A-CEA) was used as a measuring instrument. It is a measure of self-report that measures to what degree students use certain coping strategies to respond to academic stress.

Results: The strategy most commonly used by medical students is the search for support. This is also the most used among women followed by emotion-centered strategies, while men show a greater preference for active strategies.

Conclusions: Students use different mechanisms to overcome and process the stressful context. Coping is part of the individual psychological resources that influences on the quality of life and psychological well-being. One of the most emblematic topics in this field of study focuses on differences from a gender perspective.

Conflict of interest: No

Keywords: coping style; medical university students; academic stress; coping strategies

\section{Ethics and psychiatry}

\section{EPV0612}

\section{Advance decision to refuse treatment in anorexia nervosa. A case report.}

I. Moreno Alonso $^{1 *}$, C. García Guixot ${ }^{1}$, L. Fontecha Banegas ${ }^{1}$ and I. Cuevas ${ }^{2}$
${ }^{1}$ Hospital Universitario Principe de Asturias, Psiquiatria, Alcala de Henares, Spain and ${ }^{2}$ Hospital Universitario Principe de Asturias, Psiquiatria, Alcalá de Henares, Spain

${ }^{*}$ Corresponding author.

Introduction: An Advance Decision to Refuse Treatment (ADRT) is a written legal statement which contains treatment preferences in case the patient is declared incompetent to make a decision. This helps users to either establish or reject future treatment options. In the case of patients with anorexia nervosa, rejection can lead to malnutrition, ionic alterations and even death.

Objectives: The purpose of this study is to review the ethical debate around advance decisions in these kinds of patients, the limitation of therapeutic effort vs. the principle of charity.

Methods: A 40-year-old patient diagnosed with anorexia nervosa for over 20 years with very frequent and long-term admissions. During her last admission she requests to sign an ADRT document to limit her treatment and to not be re-nourished.

Results: The hospital's ethics board, the patient psychiatrist, palliative care and endocrinology members met to discuss the situation. Conclusions: During the meeting, there was a debate involving the patient's autonomy vs. the principle of charity. Due to the fact that this is a chronic illness involving several relapses, the chances of complete recovery were scarce. Therefore, the patient's will was respected and she was offered palliative treatment.

Conflict of interest: No

Keywords: 'TCA'; 'ethical'; 'advance decision'

\section{EPV0620}

\section{Restraint in psychiatry: the perception of caregivers}

N. Messedi ${ }^{\star}$, F. Charfeddine, L. Aribi and O. Amami

Hédi Chaker university Hospital, Department of Psychiatry (b), Sfax, Tunisia

${ }^{\star}$ Corresponding author.

Introduction: The practice of restraint in psychiatry has been steadily increasing in the past few decades. What about caregivers' perception and feelings when it becomes the last therapeutic resort?

Objectives: study caregiver's experience and feelings facing restraint and its impact on caregiver-patient relationship.

Methods: A cross-sectional descriptive study among 38 doctors and 30 nurses was performed on June 2019 in the 3 departments of psychiatry, Hedi Chaker University Hospital, Sfax in Tunisia. We used anonymous questionnaire exploring their feelings, perception facing restraint, and impact on caregiver-patient relationship.

Results: The average age of participants was 35.19 years; the sexratio $(\mathrm{H} / \mathrm{F})=0.38$. The mean years of exercise in psychiatry was 2.52 for residents, 7.38 for psychiatrists and 9.07 for nurses. When describing patients' feeling, caregivers identified anger in $60.3 \%$ and dehumanization in $32.4 \%$ cases. Satisfaction was described by $43.3 \%$ from nurses and only by $5.3 \%$ of doctors. Both of them describe also fear with respectively $20 \%$ and $7.9 \%$, but at the same time, this procedure give security especially for patient $(82.4 \%)$. Restraint increase caregiver's availability which make a trustful relationship perceived by $61.8 \%$ for doctors. However, for $54.4 \%$ of physicians, restraint leads to patient obedience for fear of being attached again. The use of restraint was abusive according to $33.8 \%$, the main reason was related to lack of resources for $30.8 \%$ of them. 
Conclusions: Our study shows a rich emotional experience related to the practice of restraint. The best way to prevent abusive restraint and painful experiences seems to be the ongoing questioning of practices.

Conflict of interest: No

Keywords: restraint; feelings of caregivers; Psychiatry; patients' impact

\section{EPV0621}

\section{Restraint in psychiatry: assessing the knowledge of nurses and trainee doctors}

N. Messedi ${ }^{\star}$, A. Chamseddine, L. Aribi, F. Charfeddine and O. Amami

Hédi Chaker university Hospital, Department of Psychiatry (b), Sfax, Tunisia

${ }^{\star}$ Corresponding author.

Introduction: Physical restraint is a common practice in psychiatry, yet little explored in literature. Nurses and doctors in training had participated usually in experiments of restraint, and had a crucial role for its practice and its efficiency.

Objectives: To assess the knowledge and practice of nurses and trainee doctors towards physical restraint.

Methods: This is a cross-sectional, descriptive and comparative study among 30 nurses and 26 trainee doctors practicing in the psychiatric departments of university Hospital in Sfax, Tunisia. We used anonymous questionnaire exploring practical and legal knowledge about physical restraint.

Results: The most common indications were: aggressiveness against other persons (78.6\%), self-aggressiveness (55.4\%), and agitation (62.5\%). According to $43.3 \%$ of nurses, restraint can be performed even without medical prescription, which is not true for trainee doctors (15.4\%). The restraint should be performed if it is the only way to prevent immediate harm to the patient or others in $56.7 \%$ of nurses and $69.7 \%$ of trainee doctors. Trainee doctors regularly inform each patient about reasons for being restrained in $46.2 \%$ of case and nurses in $63.3 \%$. They identified the overwork $(23.3 \%)$ and patient's inability to discern $(20 \%)$ as reasons for lack of information transmitted to patients. The use of restraint is abusive for $40 \%$ of nurses and $23.1 \%$ of doctors.

Conclusions: This study showed some important misunderstandings of nurses and trainee doctors about using physical restraint. Findings of this study serve as a supporting reason for importance of educating psychiatric staff about the use of physical restraint, especially to avoid its misuse.

Conflict of interest: No

Keywords: Physical restraint; nurses and trainee doctors'

knowledge; practice towards physical restraint

\section{Forensic psychiatry}

\section{EPV0629}

\section{Suspicion of chemical submission in a hospital emergency department: a case report}

D. Rentero Martin ${ }^{1 \star}$, A. Parra Gonzalez ${ }^{1}$, B. Zamora Banegas ${ }^{2}$, V. Santana Florido ${ }^{2}$ and J.L. Eguía Barbarin ${ }^{1}$
${ }^{1}$ Hospital 12 de Octubre, Psiquiatría, Madrid, Spain and ${ }^{2}$ Hospital 12 de Octubre, Psiquiatría, madrid, Spain

${ }^{*}$ Corresponding author.

Introduction: Chemical submission (CS) is a term used to describe incidents of violence assault in which the victim experiences changes in the level of consciousness or in its ability to judge, as a result of drug or alcohol consumption.

Objectives: To present a case which took place in 12 de Octubre Hospital emergency department.

Methods: A 63 years old woman, without psychiatric antecedents, arrived to emergency unit with a variety of symptoms: wiziness, sedation, palpitations as well as amnesia of episode. At that time, the CS protocol was launched. Detailed history was performed. Blood and urine samples were taken. The patient denied active substance use and had only prescribed $0.5 \mathrm{mg}$ per day alprazolam in her treatment regimen.

Results: In the blood test, no significant changes were observed, but in the urine test, positivity was obtained for amphetamines, methyldioxymethamphetamine, methamphetamine and benzodiazepines. After a period of observation, the patient remembered that she went to a bar with some roommates who had been insisting all night to drink liquids and she believes that they wanted to take money from its bank account.

Conclusions: Although there are several chemical submission protocols, the best strategy to identify it early is having a high clinical suspicion of it. Occasionally, it may be confused with any psychotic disorder. To avoid this, it is necessary to verify the story with a toxicological analysis and, if in doubt, conduct an interconsultation to psychiatry department.

Conflict of interest: No

Keywords: Drugs; forensic; Chemical submission; Toxicology

\section{Intellectual disability}

\section{EPV0665}

Depression in patients with mental retardation: a case report

\section{S. Dehanov ${ }^{\star}$ and T. Ferreira}

Hospital Prof. Doutor Fernando Fonseca, Psychiatry Department, Amadora, Portugal

${ }^{*}$ Corresponding author.

Introduction: Depression in patients with mental retardation (PWMR) is a relatively common condition in this population. Nonetheless, studies in this group about the diagnosis and treatment of depression and other psychiatric conditions are relatively scarce. Typically, the criteria of classification systems can be applied to this population, although some specificities may be common, leading to diagnostic questions.

Objectives: To describe and discuss a case report of a patient with mental retardation and depression

Methods: Review of the clinical case and literature

Results: A 56-year old woman with previous history of depressive episodes was observed in a psychiatric appointment due to complaints of restlessness, memory loss, reduction of selfcare and trouble in completing activities of daily living. Although not having an official diagnosis of mental retardation, the patient had a history 
of difficulties during the academic period, having only completed the primary school with two failed years due to difficulties in learning. During the observation, the memory complaints were the most prominent symptom, conditioning the patient's daily life activities. Treatable causes of dementia were excluded, and the patient had clinical features of pseudodementia (depressive symptoms prior to the memory difficulties, 'don't know' responses, and a past history of memory difficulties during previous depressive episodes). Treatment with sertraline was initiated and titrated to $150 \mathrm{mg}$ /day with progressive improvement.

Conclusions: This case illustrates some of the challenges of the phenomenology, diagnosis and treatment of depression in PWMR. Despite not being an uncommon condition in this population, there are few case reports of its clinical presentation and treatment.

Conflict of interest: No

Keywords: mental retardation; Dépression

\section{Mental health care}

\section{EPV0689}

Evaluation of a coordination program between mental health and primary care

I. Diaz-Quero*, B. Mata-Saenz, V. Muñoz-Martínez, V. QueroPalomino and L. Beato-Fernandez

Hospital General Universitario de Ciudad Real, Psychiatry, Ciudad Real, Spain

${ }^{*}$ Corresponding author.

Introduction: Nowadays, the treatment of patients with mental illness is carried out at community level, the collaboration between Mental Health and Primary Care has become something essential in our Unit. This is why we have created a coordination program between both services to facilitate communication through learning sessions, co-therapy and the use of new technologies.

Objectives: The aim was to evaluate the results of the implementation of our coordination program.

Methods: An observational, analytical study was performed with a sample of patients derived from Primary Care and attended in 42 months. Variables were collected in a database and analyzed with the SPSS 19.0.

Results: Our sample includes 2956 patients. $25.7 \%$ did not attend the assessment, $50 \%$ were discharged at first or second assessment, $4.9 \%$ are currently undergoing evaluation, and $4.1 \%$ have abandoned treatment. 78 visits have been made to 19 health centers in order to take part in the training of general practitioners (GPs) in detection and management of psychiatric problems, as suicidal behaviors, and assessment about specific patients. 140 consultations have been attended via email or telephone, with 34 preferential consultations. A total of 8 home visits have been made to 3 patients in situations of greater vulnerability.

Conclusions: It will be necessary to evaluate changes in the level of satisfaction after adding the suggestions made by GPs and to include every patient in the program (at the moment only $60 \%$ of them are firstly attended by the professional who coordinates with GPs).

Conflict of interest: No
Keywords: mental health care; epidemiology and social psychiatry; Quality Management

\section{EPV0700}

Stigmatizing attitudes of mental health professionals and their familiarity with mental disease.

F. Del Olmo ${ }^{1 *}$, A.C. Martinez ${ }^{2}$, M. Gonzalez-Blanco ${ }^{3}$ and C. Rebolleda ${ }^{4}$

${ }^{1}$ CLINICA SAN MIGUEL, HERMANAS HOSPITALARIAS, Medical Director, Madrid, Spain; ${ }^{2}$ CLINICA SAN MIGUEL, HERMANAS

HOSPITALARIAS, Crps Arturo Soria, Madrid, Spain; ${ }^{3}$ CLINICA SAN MIGUEL, HERMANAS HOSPITALARIAS, MrAravaca, MADRID, Spain and ${ }^{4}$ CLINICA SAN MIGUEL, HERMANAS

HOSPITALARIAS, Crps Aranjuez, ARANJUEZ, Spain

${ }^{*}$ Corresponding author.

Introduction: Research show mental health professionals share some social prejudices, as they have stigmatizing attitudes or behaviours towards mental illness. Studies on general public suggest that prior personal contact or familiarity with mental illness correlates with less stigmatizing attitudes, which has led to design strategies for stigma reduction based on contact with users. However, similar studies in mental health professionals show conflicting results, which has been related to either a differential effect of contact on professionals, or to the non-distinction between professional and personal contact.

Objectives: A study was designed to determine the relationship between attitudes towards, and the level of familiarity with, mental illness, in mental health professionals.

Methods: Professionals from centres belonging to Hospital Sisters of Spain, Portugal and Italy entered the study. CAMI scale was used to measure attitudes towards mental illness. It includes both "negative" (authoritarianism and social restriction) and "positive" (benevolence and community mental health support) attitudes. Level of Contact Scale was used to measure prior contact with mental illness. It has 11 items, three measuring personal contacts.

Results: 1525 professionals completed the questionnaires. A statistically significant but weak correlation $(\mathrm{r}<0.2)$ was found between familiarity and attitudes in the expected direction (previous contacts with positive attitudes and vice versa). In addition, subscale of personal contacts correlated even weaker $(r<0.1)$ or not significantly.

Conclusions: The results suggest that previous contact or personal familiarity of mental health professionals with the mental illness is only slightly related to their attitudes towards it.

Conflict of interest: No

Keywords: Stigma; attitudes; Mental Health Professionals

\section{Neuroimaging}

\section{EPV0748}

Is it mandatory to perform systematically a brain computed tomography in first-episode psychosis?

J.A. Nova Marqués*, D. Hernández Huerta and E.B. Alonso Cano 
University Hospital Ramon y Cajal, Psychiatry Department, Madrid, Spain

${ }^{*}$ Corresponding author.

Introduction: Recommendations for brain imaging in first-episode psychosis (FEP) have been incorporated into practice guidelines although considerable disparity remains about whether scanning should be routinely undertaken (Forbes et al., 2019).

Objectives: Review the radiological findings of brain computedtomography (CT) of FEP admitted to psychiatry inpatient unit.

Methods: Retrospective study of FEP admitted to psychiatry inpatient unit in a tertiary hospital (Spain) from 01/01/2017 to $31 / 01 / 2018$. Descriptive analysis of gender, age, length of stay, DSM-5 diagnosis and classification of radiological findings into four numbered groups (according to Goulet et al., 2009) with the statistical program IBM SPSS v.22.

Results: 36 patients were included; $26(72.2 \%)$ were males, the mean age was 39.36 (SD:15.254) and the average length of stay was 12.92 (SD: 8.443 ) days. Regarding diagnosis $33.3 \%$ were brief psychotic disorder, $25.0 \%$ unspecified psychotic disorder, $19.4 \%$ substance induced psychotic disorder, $16.7 \%$ schizophreniform disorder, $2.8 \%$ schizophrenia and $2.8 \%$ delusional disorder. With respect to radiological findings, $32(91.4 \%)$ brain CT were classified as 1 group, 1 (2.9\%) as 2 group and $2(5.7 \%)$ as 3 group. None was classified as 4 group. One patient did not have brain CT.

Conclusions: Only 3 (8.6\%) patients had radiological findings and none of them justified the psychotic symptoms. All of these findings were found in patients older than 50 years. This results are in line with recent investigations (Coentre et al., 2016, Forbes et al., 2019) that suggest brain neuroimaging in FEP should be reserved for patients with neurological/organic signs, atypical clinical presentation or old age ( $>50$ years).

Conflict of interest: No

Keywords: neuroimaging; first-episode psychosis; brain computed-tomography

\section{Neuroscience in psychiatry}

\section{EPV0783}

\section{Neurofeedback to treat mild cognitive decline: what scientific literature says}

\author{
M. Suárez-Gómez ${ }^{1 \star}$, A. Suárez-Gómez ${ }^{2}$ and F. Suárez-González ${ }^{3}$ \\ ${ }^{1}$ Unidade Local de Saúde do Baixo Alentejo, Psychiatry, Beja, Portugal; \\ ${ }^{2}$ Centro de Saúde, Comunitary Nursery, Borba, Portugal and ${ }^{3}$ Centro \\ de Salud San Roque, Practice Medicine, Badajoz, Spain \\ ${ }^{*}$ Corresponding author.
}

Introduction: Neurofeedback is an effective, noninvasive technique used mainly in anxiety and problems with hiperactivity and attention. Objectives: To know the clinical befefits in support treatment with Neurofeedback of old age people with mild cognitive decline.

Methods: To do a syatematic review of the recently scientific literature at main database such as Pubmed, Medline, Chocrane, Scopus. Results: This review describes the protocol most frequently used with Neurofeedback for the treatment of old age people with mild cognitive decline and the results found about clinical benefits, side effects and costs. In most studies, benefits are seen from the sixth training session, with each season during about 20 minutes, increasing brainwave speed with a video game that feeds brain activity. Neurofeedback is a not invasive therapeutic support well tolerated with no side effects and a good economical perfil, but more used on private context.

Conclusions: Neurofeedback is an effective, noninvasive technique used to treat mild cognitive decline with satisfactory results, allowing changes associated no with dementia not to be as rapid or acute and less related effects, improving quality of life of the subjects.

\section{Conflict of interest: No}

Keywords: neurofeedback; Mild cognitive impairment; old age psychiatry; treatment

\section{Obsessive-compulsive disorder}

\section{EPV0786}

\section{Neurological soft signs in obsessive-compulsive disorder}

\section{Fernandes Santos ${ }^{1 \star}$ and I. Canelas Da Silva ${ }^{2}$ \\ ${ }^{1}$ Hospital Garcia de Orta, Department of Psychiatry And Mental Health, Almada, Portugal and ${ }^{2}$ Hospital Vila Franca de Xira, Department of Psychiatry, Vila Franca de Xira, Portugal ${ }^{\star}$ Corresponding author.}

Introduction: Neurological soft signs (NSS) are minor nonlocalizing neurological abnormalities without diagnostic specificity, involving defects in motor coordination, sensory integration and primitive reflexes, normally present in the course of early development but considered abnormal when present beyond childhood. NSS, indicative of subtle brain dysfunction, have been described in a variety of psychiatric entities, such as schizophrenia spectrum disorders and bipolar disorder. There is evidence linking neurological dysfunction to obsessive-compulsive disorder (OCD), but NSS have been inconsistently reported.

Objectives: Our aim is to review current knowledge on the presence of NSS in OCD patients and their potential clinical importance.

Methods: Non-systematic review of literature through search on PubMed/MEDLINE electronic database for publications up to 2019, in English, following the terms "neurological soft signs" and "obsessive-compulsive disorder".

Results: The majority of studies indicate that OCD patients exhibit significantly higher rates of NSS on both sides of the body, with impairments in multiple domains: motor coordination, sensory integration, complex motor tests, right/left and spatial orientation, and hard signs. NSS positively correlate with the severity of disease, measured with The Yale-Brown Obsessive Compulsive Scale, and the presence of alexithymia and neuropsychological deficits. Left-sided visuospatial soft signs were intensified in pharmacological treatment non-responders compared to responders. OCD patients with poor insight appear to have higher NSS scores than those with good insight, with more impaired neurological motor performance.

Conclusions: Evidence supports that patients with OCD have increased rates of diverse NSS. OCD patients with NSS may have a more complicated expression of symptomatology, requiring greater clinical care.

\section{Conflict of interest: No}

Keywords: obsessive-compulsive disorder; neurological soft signs 


\section{EPV0787}

\section{Refractory obsessive-compulsive disorder. A case report.}

C.M. Franch ${ }^{1 \star}$, R. Gómez Martínez ${ }^{1}$, A. Serrano García ${ }^{1}$, E. Calzada Miranda $^{1}$, A. Ugidos Fernandez ${ }^{2}$ and A. Alonso De La Torre ${ }^{2}$

${ }^{1}$ CAULE, Psychiatry, Leon, Spain and ${ }^{2}$ CAULE, Psychiatry, León, Spain

${ }^{\star}$ Corresponding author.

Introduction: A single 22 year-old-single man. Psychiatric history from the age of 15. Clinical start due to alterations of the circadian rhythm linked to video-games, as well as different obsessions and compulsions, being diagnosed of Obsessive Compulsive Disorder. Objectives: He required multiple admissions, both in Adolescent Psychiatry Unit and in Acute Unit of Psychiatry due to anxious symptoms, obsessive thoughts, compulsions, dysmorphophobicsomatic ideation associated with sexual functioning and alteration of male hormones, and suicidal gestures. He had also performed therapy in different devices, such as Day Hospital and Convalescence Unit.

Methods: He strongly resists to psychopharmacological treatment, due in part to the different side effects actually suffered with diferents drugs (being clear the high sensitivity of the patient to neuroleptics and stabilizers) and partially influenced by close to delirious phase beliefs about his problems in the sexual/hormonal areas.

Results: Last years' evolution show a deterioration in academic functioning (abandonment of studies), work (inability to keep a job) and even in social and family networks, reaching a point where home management has been impossible because of the continuous demands of analytical-hormonal tests, and behavioral alterations because of the refusal to yield to their demands.

Conclusions: Given this situation, and in agreement with the family, it is decided to seek new treatment alternatives, so the case is brought to the attention of the Reference Neurosurgery Service for Obsessive Compulsive Disorder surgery, being the case studied and accepted by the same one and being in these moments realizing psychosurgery preoperative tests.

Conflict of interest: No

Keywords: Refractory; Psychosurgery; Neurosurgery; ocd

\section{EPV0788}

\section{Depression or obsessive compulsive disorder suicidal subtype? A case report.}

M. Jiménez Cabañas*, M. Sánchez Amores, L. Chiva San Román, M. Huete Naval, V. Pérez Navarro and C. Carrascosa Carrascosa

Hospital Universitario Clínico San Carlos, Instituto De Psiquiatría Y Salud Mental, Madrid, Spain

${ }^{\star}$ Corresponding author.

Introduction: We describe the case of a 21 year old women, without personal or family psychiatric history, who has been with low mood, high anxiety, conciliation insomnia, decreased appetite, weight loss and social withdrawal. Persistent thoughts of suicide, which are egodystonic, intrusive and rumiatives, are present since she was 15 years old. She also presents impulse phobia and fear of losing control which, together with the longstanding autolytic idea, has created the important discomfort nowadays. We consider that the possible diagnosis could be major depressive disorder (MDD) or obsessive compulsive disorder (OCD) of suicidal type.

Objectives: To review the literature of MDD and suicidal OCD, study the differences between both and finally, make a differential diagnosis.

Methods: Literature review of scientific papers searching in Pubmed. We considered papers in English.

Results: Suicidal OCD is a disorder in which the patient present thoughts related to suicide and they can also present compulsions that try to neutralize the discomfort generated by those thoughts. Patients with this type of disorder believe that they will finally carry out these ideas. For this reason, they are insecure and afraid. Like any type of OCD, it is closely related to depression. However, in this subtype, due to the content of their thoughts, it is difficult to assess whether suicidal ideation is a consequence of depression or if, on the contrary, depression derives from maintaining uncontrollable, irrational and egodystonic thoughts for a long time.

Conclusions: OCD is a difficult disorder to diagnose and differentiate from MDD. Therefore, it is an underdiagnosed disorder.

Conflict of interest: No

Keywords: obsessive-compulsive disorder; ocd; Suicidal OCD; Suicide

\section{EPV0791}

\section{Effect of family dynamics in patients with severe obsessive-compulsive disorder.}

\section{P. García-González ${ }^{*}$ and E. Rodríguez Muro \\ Hospital de Día de Villaverde, Psychiatry, Madrid, Spain ${ }^{\star}$ Corresponding author.}

Introduction: Villaverde's day hospital is a psychotherapeutic treatment Center for adult patients (from 18 to 65 years old) with severe mental disorder. It is an intensive treatment center focused mainly in group therapy, with a therapeutic community approach. Our patients are not referred by diagnosis but by the little improvement they have with ambulatory treatment so they are usually chronic, treatment-resistant cases.

Objectives: A small proportion of our cases have ObsessiveCompulsive Disorder (OCD) as a main diagnosis. One of the usual demands we receive from these patients is a worsening of their OCD symptoms without a clear cause. We have observed that, after addressing this worsening in different therapies, it usually had some relation to complicated family dynamics so we wanted to study how these dynamics affected their symptoms.

Methods: We selected cases with severe treatment-resistant OCD treated in our day hospital during 2018 and 2019 to analyze their family dynamics and how changes on this dynamics affected their symptoms.

Results: In this period, $9,8 \%$ of our patients had OCD as main diagnosis. All the cases we received were affected by different maladjusted family dynamics and their symptoms improved once these where addressed in different ways (group and individual therapy, family interviews).

Conclusions: Treatment-resistant OCD patients pose an important challenge to mental health professionals. In many cases we tend to focus on their symptoms, disregarding their family dynamics. Addressing their social context and these dynamics may improve their symptoms and quality of life. 
Conflict of interest: No

Keywords: DAY HOSPITAL; obsessive-compulsive disorder; systemic therapy

\section{Old age psychiatry}

\section{EPV0798}

Is exercise effective in reducing symptoms of depression in alzheimer's dementia? a systematic literature review

L.V. Banerjee ${ }^{1 *}$ and S. De Souza ${ }^{2}$

${ }^{1}$ University of Bristol, Medical School, Bristol, United Kingdom and

${ }^{2}$ Somerset Partnership NHS Foundation Trust, Old Age Psychiatry,

Taunton, United Kingdom

${ }^{\star}$ Corresponding author.

Introduction: An estimated 46.8 million people live with Dementia worldwide. Approximately $30 \%$ of patient's with dementia will suffer with a major depressive disorder. Treatment with antidepressant medication can be problematic in older adults with dementia, and engagement with psychological therapy may be more challenging. Therefore, we undertook a review of the literature for evidence that exercise could reduce the symptoms of depression in Alzheimer's Dementia.

Objectives: To establish if there is an evidence base for the treatment of depressive symptoms in Alzheimer's dementia.

Methods: A literature search was undertaken in July 2019. Papers were identified from the Medline database. Search terms focused on the disease (Alzheimer's dementia), depression and exercise and its synonyms. The search was limited to papers from after 1999, English language and randomised trials.

Results: Using the criteria above a total of 13 papers were identified. 3 papers were excluded on initial screening. Full text was accessed for the remaining 10 papers. Of these 5 of these papers were excluded as they were not limited to Alzheimer's Dementia. The remaining 5 papers were analysed. The results of these papers were variable, with little evidence of significant improvement in depressive symptoms in dementia.

Conclusions: Currently there is a lack of evidence to recommend treatment of depressive symptoms within a dementia, with exercise. However, given the neurochemical changes associated with exercise there is good reason to believe that exercise may well improve these symptoms. Our study should be a call to arms. It is imperative there are further studies are need in the area.

Conflict of interest: No

Keywords: dementia; Dépression; Exercise; treatment

\section{EPV0808}

Charles bonnet syndrome as the first stage of dementia development. A case presentation.

R. Manzanero Estopiñan ${ }^{\star}$ and C. Pérez Da Silva

University Hospital Ramon y Cajal, Psychiatry Department, Madrid, Spain

${ }^{*}$ Corresponding author.

Introduction: Charles Bonnet Syndrome is characterized by visual hallucinations in the absence of cognitive impairment, and often associated with visual deficits. Some risk factors for its development are advanced age, optic pathway injuries, social isolation, depression, or sleep disturbances.
Objectives: Review of the relationship between CBS and the development of dementia.

Methods: Illustrative case report. Bibliographic search in PubMed about the link between CBS and dementia. Introduced terms: CBS, dementia, Lewy Body Dementia (DLB).

Results: A 93-year-old women with history of dysthymia, was referred for complex visual hallucinations since several months, associated with behavioral impact, disturbing her sleep. There were no other psychiatric or cognitive symptoms. The patient also had marked visual impairment due to bilateral macular degeneration. In CT study is detected chronic microangiopathy and diffuse bilateral cortical-subcortical atrophy. Given the absence of other psychopathology, CBS was the accepted diagnosis, and low-dose antipsychotic treatment was introduced. Initially impact of the hallucinations diminished and insight was gained. At follow-up, she developed Parkinsonism, repeated falls, cognitive impairment, and auditory hallucinations, being now the diagnosis compatible with LDB. LDB core features are progressive memory deficits, Parkinsonism and repeated falls. Due to its frequent debut with isolated visual hallucinations, on cross-sectional observation both entities can be misdiagnosed, with therapeutic consequences.

Conclusions: Careful longitudinal observation is recommended to exclude DLB in elderly patients with CBS, being useful the detection of neuropsychological changes distinctive of the early stages of DLB. Currently, the nature of the relationship between the two entities continues to be uncertain.

\section{Conflict of interest: No}

Keywords: Charles-Bonnet syndrome; Lewy body dementia; case report; visual hallucinations

\section{EPV0812}

Use of visual hallucinations characteristics to help in the differential diagnosis between charles bonnet syndrome and lewy body dementia initial stage.

\section{Pérez Da Silva ${ }^{1 \star}$ and R. Manzanero Estopiñan ${ }^{2}$}

${ }^{1}$ University Hospital Ramon y Cajal, Psychiatry Department, Madrid, Spain and ${ }^{2}$ Hospital Universitario Ramon y Cajal, Psychiatry, Madrid, Spain

${ }^{*}$ Corresponding author.

Introduction: Lewy body dementia (DLB) is the second most prevalent neurodegenerative dementia. However, it is often not detected or misdiagnosed. DLB diagnostic criteria (McKeith et al.2017) list four core items: visual hallucinations, motor parkinsonism, cognitive fluctuations, and REM sleep disorders. However, it often debuts with isolated visual hallucinations, leading to misdiagnosis with Charles Bonnet Syndrome (CBS), which consists in visual hallucinations in the absence of other psychopathological alterations Objectives: Evaluate whether visual hallucination characteristics can help to make an early differential diagnosis between CBS and LBD.

Methods: Research in PubMed of literature about the characteristics of visual hallucinations. Terms introduced: LBD, CBS, visual hallucinations, diagnostic criteria.

Results: Both entities include complex visual hallucinations. They usually last minutes in LBD while in CBD can be longer and more frequent. Whilst in CBS consciousness state is always preserved, other sensory disturbances are not present and hallucinations are experienced as unreal, lacking affective impact, in LBD these aspects can present altered: visual hallucinations usually appear related to times of wakefulness decreased level, they are often 
polymodal (the most distinguishing characteristic), with associated affective impact, altering reality judgment.

Conclusions: Because of the different presentation of visual hallucinations in LBD, it is not possible to make an accurately early clinical diagnosis in the absence of other symptoms, although some data may increase suspicion degree fot the diagnosis. We recommend close follow up in CBS cases with particular attention to LBD other symptoms.

\section{Conflict of interest: No}

Keywords: visual hallucinations; differential diagnosis; CharlesBonnet syndrome; Lewy body dementia

\section{EPV0815}

\section{Communication skills with the elderly patients}

M. Suárez-Gómez ${ }^{1 *}$, F. Suárez-González ${ }^{2}$ and A. Suárez-Gómez ${ }^{3}$

${ }^{1}$ Unidade Local de Saúde do Baixo Alentejo, Psychiatry, Beja, Portugal;

${ }^{2}$ Centro de Salud San Roque, Practice Medicine, Badajoz, Spain and

${ }^{3}$ Centro de Saúde, Comunitary Nursery, Borba, Portugal

${ }^{\star}$ Corresponding author.

Introduction: adults over 65 years of age have special characteristics, since they are usually patients with great comorbidity and polymedics. Their sensory and functional limitations make them dependent on third parties, even to communicate with the doctor, which can lead to a difficulty in understanding the way they should take the medication, In addition, they may often feel not enough confidence to talk about problems such as loneliness, depression, fear of death, memory loss, alcohol abuse or abuse.

Objectives: To know the most effective communication techniques between the psychiatrist and the elderly patients to improve medical attention.

Methods: A literature review of the last 5 years was carried out in the main medical search.

Results: The literature shows that often the patient and the caregiver do not understand the information about the treatment. In addition, the doctor usually addresses the caregiver, rather than the patient. The social circumstances of the elderly may also be related to the communication problems.

Conclusions: An adequate relationship between the psychiatrist and the patient improves the patient's adherence to the treatment. It is important that the doctor directs the conversation to the patient and not only to the caregiver. The doctor must create the right environment so that the patient can talk about their problems in the time they need, always guaranteeing confidentiality. It is important to consider social aspects, assess sensory and functional limitations, involve caregivers in care and ensure coordination with the team of Primary care and the other specialists.

Conflict of interest: No

Keywords: Geriatric Psychiatry; Adherence; Communication; old age

\section{EPV0834}

Neuropsychiatric symptoms in a intracranial lipome: a clinical case

\footnotetext{
M. Suárez-Gómez ${ }^{1 \star}, \mathrm{P}$. Gonçalves Pereira ${ }^{2}$ and P. Rodrigues ${ }^{1}$

${ }^{1}$ Unidade Local de Saúde do Baixo Alentejo, Psychiatry, Beja, Portugal and ${ }^{2}$ Algarve Medical School, Portugal, Neuroradiology, Faro, Portugal ${ }^{\star}$ Corresponding author.
}

Introduction: The term lipoma leads us to think that we are facing a neoplasm, but in reality it is an embryological defect with neuropsychiatric clinics in about one third of case.

Objectives: To present a clinical case about an old age patient follow up in the geriatric psychiatry team at Local Health Unit of Baixo Alentejo in Portugal, who describes headaches and depressive symptoms and in whom is found a intracranial lipoma in neuroimaging test. To analyze the Clinical and epidemiological relation among psychiatric, neurological symptoms and intracranial tumors.

Methods: It is presented the clinical case of a 68 years old man who is sent to the geriatric psychiatry team describing depressive symptoms and headaches without previous personal or familiar story in psychiatry and neurology. After neuroimaging test, it is found an small intracranial pericallosal lipoma. It was performed a neurpsycological and genetic tests. Then, authors present a review of recent scientific literature of the last 5-years about the relation among psychiatric, neurological symptoms and intracranial lipoma. The results were collected from databases (Pubmed, Cochrane and Scopus).

Results: Half of the Intra-cranial lipomas are pericallosal. Clinical manifestations include headache (25\%), epilepsy (30\%), mental disorders (15\%), one third being asymptomatic and normal neurological examination. Indeed, the quadrigeminal lipoma had no clinical expression, being those of this location prone to cause ataxia, hydrocephalus, gaze paralysis or enveloping the trochlear nerve.

Conclusions: It is important to know psychiatric manifestations of neurological syndromes and to do a good diferential diagnoses to better enhance the Clinical and therapeutic approach.

\section{Conflict of interest: No}

Keywords: neuroimaging; lipoma; old age psychiatry; Neuropsychiatric symptoms

\section{Oncology and psychiatry}

\section{EPV0846}

\section{Interventions on distress in terminally ill cancer} patients: the state of the art

\author{
S. Ramos ${ }^{1 \star}$ and M.A. Tavares ${ }^{2}$ \\ ${ }^{1}$ Unidade Local de Saúde da Guarda, E.P.E., Psychiatry, Guarda, \\ Portugal and ${ }^{2}$ Instituto Português de Oncologia do Porto FG, EPE, \\ Psycho-oncology, Porto, Portugal \\ ${ }^{\star}$ Corresponding author.
}

Introduction: Cancer is the second leading cause of the death worldwide - it has been responsible for approximately 9.6 million deaths in 2018. Psychological and existential distress affects up to $49 \%$ of cancer patients and is often experienced by the terminally ill. To ameliorate such suffering, psychologic and psychiatric support is often requested. Such a particular time of a patient's life requires equally particular interventions, which mental health professionals may be ill equipped to provide.

Objectives: We aim to review the recent advances in interventions on distress in terminally ill cancer patients.

Methods: We conducted a comprehensive non-systematic review on PubMed.

Results: We identified pharmacologic and non-pharmacologic interventions. Regarding pharmacologic interventions, classic 
psychedelics have been recently revisited with favorable results in phase II clinical trials, suggesting decreased rates of psychologic distress following single dose administration integrated in psychotherapy. Several psychotherapeutic interventions in recent years have focused on the terminally ill patient, though effectiveness studies have relayed inconsistent results and little evidence to support their utility in clinical practice.

Conclusions: There are several interventions available for existential distress in the terminally ill patient, though effectiveness results are not promising. Classic psychedelics may be a useful tool to ameliorate psychologic suffering in advanced cancer. Further studies are necessary to develop more efficient strategies.

Conflict of interest: No

Keyword: Existencial distress

\section{Others}

\section{EPV0861}

The relationship within the first job and the emotional intelligence in graduates of the health area of two universities in santa marta - colombia

A. Guardiola Esmeral ${ }^{1}$, L.F. Miranda ${ }^{2 \star}$, J. Viloria Escobar ${ }^{3}$ and K. Pérez Correa ${ }^{4}$

${ }^{1}$ Universidad Cooperativa de Colombia, Facultad Ciencias Contables Administrativas Y De Comercio Internacional, Santa Marta, Colombia; ${ }^{2}$ Universidad del Magdalena, Facultad De Educación, Santa Marta, Colombia; ${ }^{3}$ Universidad del Magdalena, Facultad De Ciencias De La Educación, Santa Marta, Colombia and ${ }^{4}$ Universidad Cooperativa de Colombia, Facultad De Psicología, Santa Marta, Colombia

${ }^{\star}$ Corresponding author.

Introduction: This research is of a non-experimental, transversal and field design; the research it is set from exploration to explanation, establishing cause-effect relationships, with a quantitative paradigm and an inductive empirical approach.

Objectives: The objective of this research is to determine the relationship between the first employment and the emotional intelligence in a group of graduates from the health area of two universities in the city of Santa Marta, Colombia.

Methods: The sample corresponds to 41 graduates from health area with geography location in the City of Santa Marta and the municipalities of Ciénaga and Fundación, Magdalena - Colombia. TMMS24 was used to assess emotional intelligence, and for the relationship between EI and the first job, a questionnaire was designed with a licker-type scale, and to measure its reliability the Cronbach's Alpha Coefficient $\mathrm{rtt}=0.92$ and The judgment of 5 experts was used. The data analysis techniques were inferential statistics, Analysis of variance (ANOVA) of one factor and SPSS statistical software.

Results: $63 \%$ of graduates meet the criteria of the Evaluation of emotional intelligence by the TMMS24. As for the analysis, it shows an adjustment of $\mathrm{R}^{2}=0.694$. The result indicates that the higher the degree of emotional intelligence there was a faster work placement than those who had a lower degree of it.

Conclusions: Therefore, graduates were located faster and have more time in the jobs mentioned; additional they assure they do not take personal positions in front of the episodes of pressure in their jobs.

Conflict of interest: No

Keywords: emotional intelligence; job; graduates; health

\section{EPV0877}

Jung and pop culture: the archetypes in harry potter.

\section{Fontecha Banegas}

Hospital Universitario Principe de Asturias, Psiquiatria, Alcala de Henares, Spain

Introduction: Carl Gustav Jung was an importante psychoanalyst whose theories regarding the collective unconscious revolutionized psychoanalytic thinking. Almost a century later, the Harry Potter books marked and accompanied a whole generation that grew up learning to pronounce complicated spells, dreaming of one day receiving the desired letter of acceptance at the Hogwarts School of Witchcraft and Wizardry. Once the "fan phase" of reading is over, many symbols can be extracted from it, finding in its story some of the main Jungian archetypes such as the person, the shadow or the self.

Objectives: To think on Jung's references present in the Harry Potter heptalogy.

Methods: For the preparation of this poster, The Archetypes and The Collective Unconscious and the seven Harry Potter books were carefully read.

Results: Abundant allusions were found to many of Jung's archetypes.

Conclusions: The reading of the classics is of vital importance in the training of future specialists. Approaching them through literatura and cinema can be a pleasant and fun way to do it.

Conflict of interest: No

Keywords: Collective Unconscious; Harry Potter; Jung; Archetypes

\section{EPV0878}

\section{Cryothermic dermatitis artefacta in adolescence.}

P. García Vázquez ${ }^{\star}$, A. Serrano García and R. Gómez Martínez

Complejo Asistencial Universitario León, Psiquiatría, Leon, Spain ${ }^{*}$ Corresponding author.

Introduction: Dermatitis artefacta, also known as factitial dermatitis, is a condition whereby self-induced skin damage is the means used to satisfy a conscious or unconscious desire to assume the sick role. The diagnosis is one of exclusion, and it is often difficult to confirm, with patients rarely admitting their role in the creation of lesions.

Objectives: This pathology is a challenge at the diagnostic level, whose clinical suspicion must be supported by an adequate history and physical examination.

Methods: A 15-year-old woman, who come to the Child Psychiatry consultations derived by the Dermatology Service, which is attended by the appearance of multiple bullous lesions throughout the body. Since August, she has reported a worsening of her mood, with feelings of loneliness and vital emptiness, with somatic and psychic anxiety referred. Finally, she recognizes that burns occur with a deodorant spray. When asked about the cause, she says he has been doubting his sexuality for several months, claiming to be homosexual for the first time. When she burns she felt pleasure and relief.

Results: Restructuring of potentially pathological personality traits of the patient was made and behavioral therapy. Psychopharmacological treatment was not necessary. After these interventions the patient improved her mood, and the burns were not repeated. 

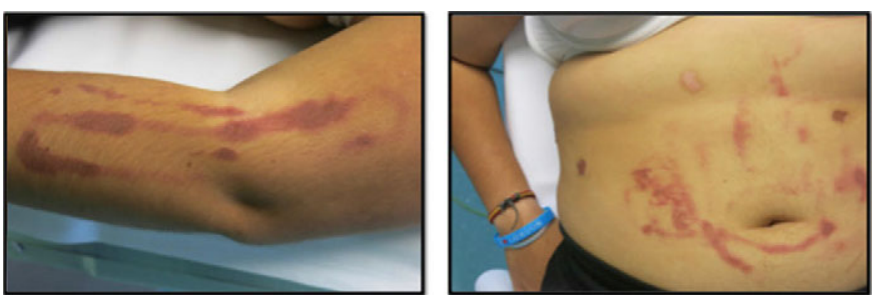

Figure 1. Transdiagnostic network of cognition and symptoms.

Conclusions: Establishing a strong physician-patient-family relationship is important in managing this condition. The prognosis of the condition is variable, but it has been shown that resolution of the underlying psychosocial stressor leads to improvement of the skin. We advocate a multidisciplinary team approachment as it has shown to improve outcomes.

Conflict of interest: No

Keywords: Dermatitis artefacta; Factitial dermatitis; adolescence; Impulsivity

\section{EPV0880}

Academic results as a predictor of automedication and suicidal ideation among medical residents.

D. Hernández-Calle ${ }^{1 \star}$, D. Carracedo Sanchidrián ${ }^{1}$, A. Cano Arenas $^{1}$ and M.F. Bravo-Ortiz ${ }^{2}$

${ }^{1}$ Hospital Universitario La Paz, Psychiatry, Madrid, Spain and ${ }^{2} \mathrm{La}$ Paz University Hospital Research Health Institute (IdiPAZ), Psychiatry, Clinical Psychology And Mental Health, Madrid, Spain

${ }^{*}$ Corresponding author.

Introduction: The association of academic results and psychopathology has been extensively studied and contradictory results have been reported. One important estimator of academic abilities in Spain is the residency entry exam, a mandatory test which result serve as a way of ordering medical students to choose the hospital in which they will perform their medical training.

Objectives: To analyse the association of the residency entry exam and the presence of automedication and suicidal ideation within the residency program.

Methods: We conducted a cross-sectional online survey directed to all medical residents in Spain and analysed the association of the order number in the residency entry exam and the presence of automedication and suicidal ideation within the residency program with a non-parametric approach (Wilcoxon-MannWhitney test).

Results: The median difference of the order number of the residency entry exam between the medical residents with and without active suicidal ideation was 45 (WMW p 0.49), 11 in the case of passive suicidal ideation (WMW $\mathrm{p}=0.75$ ) and 43 in automedication (WMW p=0.34).

Conclusions: Academic results, measured with the medical residency exam grade, is not associated with the presence of suicidal ideation or automedication in medical residents in Spain.

\section{Conflict of interest: No}

Keywords: Suicidal ideation; Medical Residents; Academic results; Automedication

\section{EPV0886}

Dissociation and confusion: the presentation of a susac syndrome. What the psychiatrist should know.

M. Pérez-Lombardo ${ }^{1 \star}$, Í. Alberdi Páramo ${ }^{2}$, A. Bermejo Pastor ${ }^{3}$, A. García Carpintero ${ }^{1}$, V. Crossley López ${ }^{1}$ and M.D. Gimeno Álvarez ${ }^{1}$

${ }^{1}$ Hospital Universitario Clínico San Carlos, Instituto De Psiquiatría Y Salud Mental, Madrid, Spain; ${ }^{2}$ Hospital Clínico San Carlos., Institute of Psychiatry And Mental Health., Madrid, Spain and ${ }^{3}$ Hospital Clínico San Carlos, Institute of Psychiatry And Mental Health, Madrid, Spain ${ }^{\star}$ Corresponding author.

Introduction: Susac syndrome is an autoimmune-mediated microangiopathy, which affects vessels from brain, retina and inner ear. The classical triad consist on encephalopathy, branch retinal artery occlusion and sensorineural hearing loss.

Objectives: The aim of this review is to outline that Susac Syndrome can make a different debut, so that we can suspect it and treat it aggressively before it causes bigger damages to the patient.

Methods: We conducted a review of the scientific literature following a clinical case registered in our center. A search was carried out on Pubmed, focused on the symptomatology, diagnosis and treatment of this disease.

Results: Susac syndrome affects women in a range of age between 20 to 40 years old ${ }^{1}$. Only $13 \%$ of patients had the typical clinical triad at the disease onset. Other symptoms are headache, cognitive impairment, confusion, emotional disturbance or behavioural changes. Some cases can also presents dissociation symptoms. Mechanisms involved in the development of the disease are still unclear. As it is thought to be an autoimmune disease, first line treatments are focussed on the immune system.

Conclusions: Susac syndrome is a rare disease whose diagnosis has increased in the past years. The early outcome of this disease is normally unspecific, with a wide range of neuropsychiatric symptoms. Suspicion on Susac Syndrome when the classical triad is not complete, ensures a faster diagnosis and an earlier treatment. Neuroimaging is essential in this disease; the presence of snowball lesions in corpus callosum is almost pathognomonic, as they are present in almost all cases.

Conflict of interest: No

Keywords: diagnosis; susac; dissociation; Magnetic Resonance Imaging

\section{EPV0891}

\section{Pathological bereavement. A case report.}

N. Serrano ${ }^{1 \star}$, M. Infante Sanchez De Lugarnuevo ${ }^{1}$, S. Guijarro ${ }^{1}$, M.D.C. Díaz Fernandez ${ }^{1}$, E. Landa ${ }^{1}$ and M.D.P. Alvites Ahumada ${ }^{2}$

${ }^{1}$ Hospital general de Tomelloso, Unidad De Salud Mental, Tomelloso, Spain and ${ }^{2}$ Complejo Hospitalario Mancha Centro, Unidad De Salud Mental Infanto Juvenil, Alcázar de San Juan (Ciudad Real), Spain ${ }^{*}$ Corresponding author.

Introduction: Pathological bereavement refers to those symptoms related to bereavement, distinguishable from depression, and which predict significant functional deterioration.

Objectives: Breravement, in the DSM-IV, is included as a "V Code" and does not recommend the diagnosis of depression, until two 
months after the loss; ICD-10 considers it an adaptive disorder and as a possible diagnosis. It is often difficult to distinguish between current and pathological bereavement. It was proposed as diagnostic criteria: presence of symptoms of intrusion, avoidance and dysfunctional adaptation, and/or somatic symptoms after one month of loss, and/or failure to form new relationships beyond 13 months later, considering the person's beliefs and socialcontext. Methods: Case report.

Results: A case report.

Conclusions: A continuum between usual and pathological bereavement is proposed, characterized by an intensification or extension of the norm. Various formal criteria are proposed such as the duration, intensity, presence of some differential symptoms and the criterion of deterioration in the functioning of the individual, not being without difficulties. Pathological bereavement has been shown to be a different stress-related disorder than others and that its symptoms are different from those of depression and anxiety-related grief. They have raised empirical criteria for diagnosing pathological bereavement in DSM 5 but there are still discrepancies that discourage this and propose to include it in other categories. All of this has led to the inclusion of Persistent Troubled Bereavement Disorder in the chapter "Affections to Continue Study".

\section{Conflict of interest: No}

Keywords: bereavement; pathological bereavement; Dépression; duel

\section{EPV0906}

Preeminence of psychopathological over other medical references in names of spanish punk groups (19812010)

\author{
F. Pavez ${ }^{1,2 *}$, E. Saura ${ }^{3}$ and P. Marset ${ }^{4}$ \\ ${ }^{1}$ University of Murcia, The International School of Doctoral Studies, \\ Murcia, Spain; ${ }^{2}$ University of Otago Wellington, Suicide And Mental \\ Health Research Group, Wellington, New Zealand; ${ }^{3}$ Centro de \\ Mediación y Terapia Familiar de Águilas, Department of Psychology, \\ Águilas, Spain and ${ }^{4}$ University of Murcia, Departamento De Ciencias \\ Sociosanitarias, Murcia, Spain \\ ${ }^{\star}$ Corresponding author.
}

Introduction: The relevance of the study of the social representations of mental disorders in the products of the popular culture has been argued previously. In a subcultural level, we hypothesize that the psychiatric field could be more present than other areas of the Medicine. Regarding the studies of the popular music, and taking in account subcultural aspects of identity affirmation, this communication addresses the references to Psychiatry and mental disorders and its comparison with other medical references in names of Punk groups (Spain, 1981-2010).

Objectives: To address the relative importance of psychiatric disorders versus other medical problems (non psychiatric) in a subcultural musical genre.

Methods: Quantitative content analysis.

Results: The names of 418 Spanish Punk groups were analyzed. $16.50 \%$ of them were associated with the Psychiatry and mental disorders versus a $5.98 \%$ for other medical references. Most of psychiatric names were associated with substance use and psychotic disorders (50.72\% and $15.94 \%$, respectively). Regarding "non psychiatric names", references to medical treatments (16\%), infectious diseases (16\%), and genital diseases (16\%) were the most frequent.

Conclusions: Psychiatric disorders are more frequent than other medical references in the names of Spanish Punk groups. It can be related to subcultural identity processes in which mental disorders work as an expression of "otherness". Despite of the existence of highly dramatic medical diseases (e.g., cancer), mental disorders remain the main focus of attention if we attend to the artistic interest expressed in our sample. For comparisons with other music genres more studies are warranted.

\section{Conflict of interest: No}

Keywords: Content analysis; Psychiatry in the arts; social representations; Culture and Psychiatry

\section{Pain}

\section{EPV0918}

\section{Years of pain in a patient with high doses of opioid} treatment: opioid induced hyperalgesia or somatic symptom disorder

\author{
A. Correa*, E. Ochoa Mangado, I. Caballero and S. Guillen \\ University Hospital Ramon y Cajal, Psychiatry Department, Madrid, \\ Spain \\ ${ }^{*}$ Corresponding author.
}

Introduction: Opioid induced hyperalgesia(OIH) is a reaction of enhanced pain perception related to the use of these drugs in the absence of disease progression or withdrawal syndrome and its different to tolerance because lack of improvement with escalation. Objectives: To describe a case showing the difficulty of differentiating Somatic Symptom Disorder (SSD) vs OIH

Methods: Illustrative case

Results: We present a case of a 45 year-old woman who was hospitalized to reduce the total opioid dose that she was taking for a severe generalized pain that limited her mobility, mostly in cervical and lumbar spine since any organic cause for her symptoms was ruled out. She also was diagnosed of Fibromyalgia and anxious-depressive syndrome in the past. She had prescribed Oxycodone at a maximum $230 \mathrm{mg} /$ day for several years in growing doses without improvement. We prescribed buprenorphine and tapered-off oxycodone, without having abstinence symptoms and experiencing a significant improvement in pain and mobility. At discharge, she was taking buprenorphine/naloxone. The final diagnosis was Opioid induced disorder and SSD. At follow-up, where she described worsening of pain and peripheral oedema, in relation to the buprenorphine, leading to its suspension, with relief of oedema but not of pain. She is having significant depressive symptoms but had not returned to her previous state when she was not able to move.

Conclusions: Chronic opioid prescription has risks of perpetuating pain, like in the OIH. Patients with psychiatric comorbidity could have more risk of having problematic use of legal opioids and differentiating both conditions may be difficult if present together. 
Conflict of interest: No

Keywords: hyperalgesia; opioid; somatic symptom disorder

\section{EPV0920}

\section{Burning mouth syndrome associated to quetiapine} treatment.

P. García Vázquez ${ }^{\star}$, A. Serrano García and R. Gómez Martínez

Complejo Asistencial Universitario León, Psiquiatría, Leon, Spain

${ }^{*}$ Corresponding author.

Introduction: Burning mouth syndrome (BMS) is a chronic condition characterized by a burning sensation of the oral cavity and is often associated with taste disturbances and xerostomia. Idiopathic or primary BMS can occur spontaneously and without any identifiable precipitating factors.

Objectives: The existence of multiple factors related to this pathology, which are involved in the appearance or maintenance is a wide field of study today.

Methods: A 67-year-old woman complained of burning pain in the tongue and oral mucosa, taste disorder, and sensory impairment. Slight improvement after treatment with Gabapentine 300mg (1-0-0). Carrier of dental prostheses, in good condition since years before the beginning of the pain. The pain is constant, with sharp characteristics. Improves when eating, the ability to taste is preserved. Subsequently, treatment with $2 \%$ lidocaine rinse (3-4 times / day) is tested, with temporary pain relief. Lorazepam $1 \mathrm{mg}$ (1-0-0), without improvement. Patient in follow-up by the Neuropsychiatry consultation for 3 years, due to major depressive disorder in treatment with Quetiapine 100mg (0-0-1).

Results: In the first consultation the treatment is modified, adding Duloxetine $60 \mathrm{mg}$ and Alprazolam $0.5 \mathrm{mg}$ and reducing the dose of Quetiapine to $75 \mathrm{mg}$ and then 50mg. After two months, she has completely removed the quetiapine, and completely disappearing the burning mouth sensation, improving his affective clinic in the same way.

Conclusions: It is a multifactorial pathology. In many cases, correcting or eliminating these etiologic agents does not improve or stop the initial symptoms, but sometimes, it does.

Conflict of interest: No

Keywords: Burning mouth syndrome; Quetiapine; Xerostomia; Psychotropics

\section{Personality and personality disorders}

\section{EPV0928}

Instinct to kill: organic personality disorder or just an antisocial brain?

\section{J. Borba Martins ${ }^{\star}$, S. Batista and A. Trindade \\ Centro Hospitalar Universitário do Algarve, Departamento De Psiquiatria E Saúde Mental - Faro, Faro, Portugal ${ }^{\star}$ Corresponding author.}

Introduction: Violence is particularly common in people with antisocial personality traits who misuse alcohol or drugs, or who have marked paranoid or sadistic traits. Organic personality disorder (OPD) is primarily characterized by change of premorbid behaviour due to an organic impairment or disease of the central nervous system.
Objectives: Presenting a case of OPD after traumatic brain injury in a man with premorbid antisocial personality traits, reflecting about the aetiology of his aggressive ideation and its potential impact in a forensic evaluation.

Methods: Case report and review of literature.

Results: A 65-year-old male was admitted to the inpatient unit with anxiety, depressed mood, suicidal and homicidal ideation and recent history of cannabinoids and benzodiazepines abuse. He has been involved in a car accident about 25 years ago, with no history of clinical follow-up. Since then, he got in conflict with almost all of his direct family members and he confesses changes in his behaviour with memory issues and increase of impulsivity and frustration intolerance recognized by his family as prior to the accident, although in a minor degree. Recent brain MRI (T2):

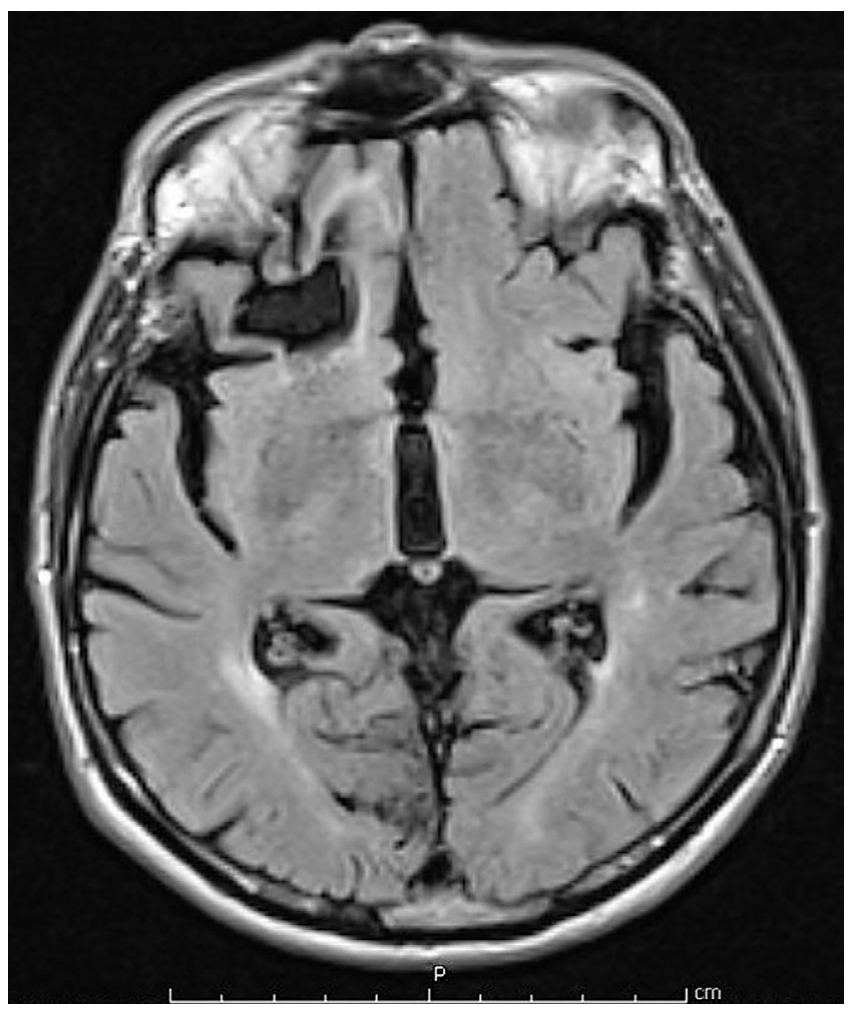

Conclusions: It is not clear whether the aggressive ideation of this patient is due to the OPD or to the premorbid antisocial traits exacerbated by the abuse of cannabinoids in the context of depression. The assessment of premorbid personality and a complete neuropsychological evaluation with follow-up after the accident would have been of uttermost importance for a forensic assessment, in order to assess his imputability.

Conflict of interest: No

Keywords: Organic personality disorder; Antisocial personality; Aggressive ideation

\section{EPV0933}

Difficulties in approaching the organic personality disorder: a case report.

A.H. Guzmán Del Castillo ${ }^{1 *}$, O. Santamaría Gómez ${ }^{1}$, A. Sánchez Gayango $^{2}$, M.P. Algarín Moriana ${ }^{3}$, E.J. López Sánchez ${ }^{2}$ and I. Martín Talavera ${ }^{1}$ 
${ }^{1}$ Hospital Universitario Nuestra Señora Virgen de Valme, Psiquiatría, Sevilla, Spain; ${ }^{2}$ Hospital Nuestra Señora de Valme, Psiquiatría, Sevilla, Spain and ${ }^{3}$ Hospital Virgen de Valme, Unidad De Hospitalización De Salud Mental, Sevilla, Spain

${ }^{\star}$ Corresponding author.

Introduction: Organic personality disorder (F07.0) as it is described in ICE-10 is characterized by a significant alteration of the habitual patterns of premorbid behaviour. The expression of emotions, needs, and impulses is particularly affected.

Objectives: Identify the difficulties involved in approaching a patient with the aforementioned diagnosis and design a multidisciplinary treatment plan that addresses the different affected areas.

Methods: We present the case of a 33 year old male, with no previous history of Mental Health (MH). Consumer of alcohol and cannabis. He presents behavioral disorders after severe TBI (left frontotemporal epidural extraxial hemorrhage and probable diffuse axonal type III lesion with subarachnoid hemorrhage in convex grooves and silviana fissure) in December 2018, leading to family conflict and denunciation of gender violence. Previously outgoing, affective and impulsive personality. Comprehensive approach in $\mathrm{MH}$ communitar y and interdisciplinary (neurology, neurosurgery and neurological rehabilitation) requiring hospitalization at 9 months with difficulties of containment of the mentioned behavioral alterations. It remains for 3 weeks until psychopathological stabilization. Treatment plan: -Pharmacological adjustment. -Family psychoeducation - Reorganization of support system.

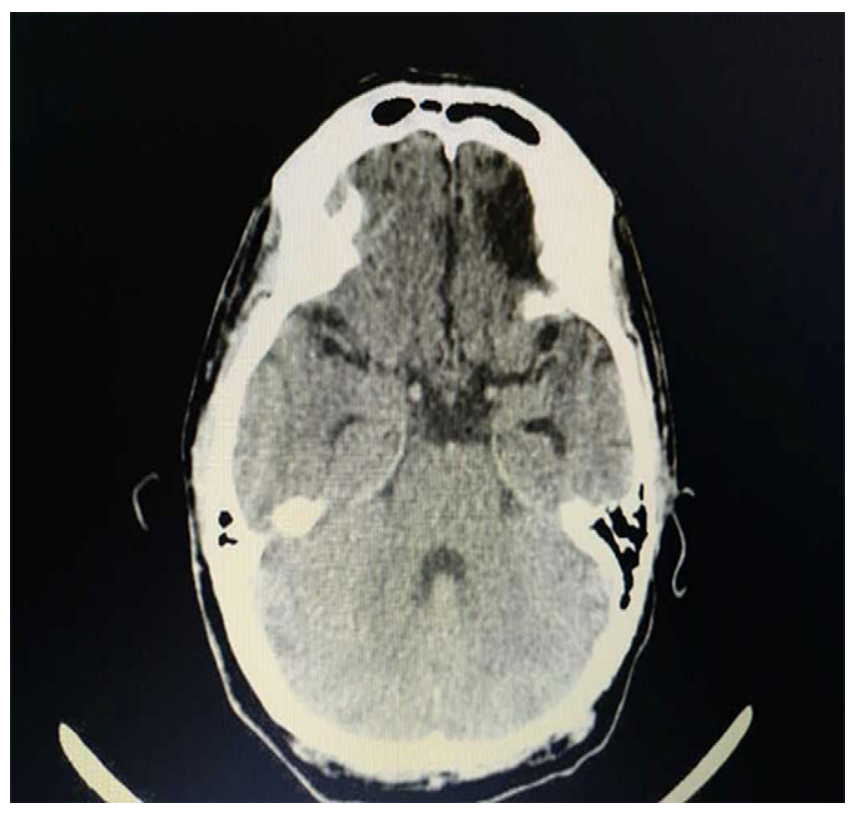

Results: We observed a clinical improvement in the patient's symptoms such a decreased number of agitation episodes. The final evaluation showed that the objectives formulated with the patient and his family were achieved.

Conclusions: The impact on the individual, family and social level of this diagnosis, as well as the therapeutic limitations and prognosis to chronicity, make necessary an specific and multidisciplinary intervention adapted to each individual case.

Conflict of interest: No

Keywords: Disorders; behavior; ORGANIC; Personality

\section{EPV0934}

\section{Is neuroticism personality trait related to neurotic disorders in hiv patients?}

D. Hernández Huerta ${ }^{1 \star}$, C. Parro Torres ${ }^{2}$, A. Madoz Gúrpide ${ }^{1}$ and E. Ochoa Mangado

${ }^{1}$ University Hospital Ramon y Cajal, Psychiatry Department, Madrid, Spain and ${ }^{2}$ Gregorio Marañón University Hospital, Psychiatry Institute, Madrid, Spain

${ }^{\star}$ Corresponding author.

Introduction: High neuroticism indexes a risk factor to the development of any common mental disorders (Jeronimus et al., 2016). Equally, this personality trait has been associated with greater risk of HIV infection and poor adherence to antiretroviral therapy (ART) (Hutton and Treisman, 2017).

Objectives: The aim of this study is to determine if neuroticism is associated with psychopathology in HIV patients.

Methods: 85 HIV patients were assessed in an observational casecontrol study. Neuroticism and neurotic disorders (major depressive disorder (MDD), dysthymic disorder, panic disorder (PD), agoraphobia, social phobia, obsessive-compulsive disorder (OCD), posttraumatic stress disorder (PTSD) and generalized anxiety disorder (GAD)) were measured with NEO PI-R questionnaire and Mini-International Neuropsychiatric Interview v5.0.0, respectively. A multivariate model (ANOVA lineal regression) was carried out with IBM SPSS v22.0. Variables included in the analysis were age, gender, economic status, educational level, alcohol dependence, other substances dependence (cannabis, cocaine, heroin), adherence to ART and the presence of neurotic disorders.

Results: $43.5 \%$ of HIV patients were diagnosed of any neurotic disorder (22.4\% PD, 17.6\% MDD, 17.6\% agoraphobia, 9.4\% social phobia, 5.9\% GAD, 3.5\% PTSD, 2.4\% dysthymic disorder). Neuroticism was significantly related to neurotic disorders $(\mathrm{p}=0.001$; $\mathrm{B}=17.747$; CI95\%:7.742-27.753) and other substances dependence ( $\mathrm{p}=0.030 ; \mathrm{B}=11.670 ;$ CI95\%:1.137-22.204).

Conclusions: High levels of neuroticism are associated with greater risk of neurotic disorders (PD, MDD and agoraphobia, mainly) and substances dependence in HIV patients. Early evaluation of this personality trait could identify an HIV patients profile at risk for psychiatric complications and/or poor adherence to ART.

Conflict of interest: No

Keywords: Personality; Neuroticism; HIV; psychopathology

\section{EPV0951}

\section{A personological dimensional perspective in pathological gambling}

A. Nirestean ${ }^{1 \star}$ and E. Lukacs ${ }^{2}$

${ }^{1}$ University of Medicine, Pharmacy, Science and Technology of Targu Mures, Psychiatry, Targu Mures, Romania and ${ }^{2}$ George Emil Palade University of Medicine, Pharmacy, Science and Technology of Targu Mures, Psychiatry, Targu Mures, Romania

${ }^{*}$ Corresponding author.

Introduction: We intend to approach this interference from a personological dimensional perspective.

Objectives: We intend to approach this interference from a personological dimensional perspective. 
Methods: We assessed the personality of the studied patients diagnosed with pathological gambling - from a dimensional perspective by means of DECAS personality inventory elaborated according to the Big Five Factors dimensional model. We observed the relations between the values of the personality dimensions and the types of pathological gambling, respectively their Clinical and evolutional characteristics. We have determined that the personality dimensions condition both the clinical symptomatology and the integration in the therapeutic strategy of the subjects. To that effect, the values of the dimension Conscientiousness have the most obvious predictive role.

Results: The delineation of the dimensional profile and its integration in the diagnostic and therapeutic approach, possible also in the absence of a categorical diagnosis of personality disorder, allows a personalized version and a more accurate one to approach the phenomenon.

Conclusions: This dimensional approach of gambling needs further and more comprehensive studies and confirmations.

Conflict of interest: No

Keywords: Gambling; dimensional perspective; Personality

\section{EPV0952}

Diagnostic profile of patients treated in villaverde's day hospital for adults

\section{P. García-González ${ }^{*}$ and E. Rodríguez Muro}

Hospital de Día de Villaverde, Psychiatry, Madrid, Spain ${ }^{*}$ Corresponding author.

Introduction: Villaverde's day hospital for adults is an intensive psychotherapeutic treatment Center for patients with severe mental disorder. It is an intensive multidisciplinary treatment center focused mainly in group therapy, with a therapeutic community approach. We receive adult patients (from 18 to 65 years old) with severe mental disorder from the southern area of Madrid. We are a nonspecific center, so our patients are not referred by diagnosis. They are usually chronic patients with little improvement with previous ambulatory treatment.

Objectives: Even without being an specific center, we have observed differences in the profile of patients referred to our center, so we wanted to study the proportion of patients with different diagnoses.

Methods: We made a qualitative analysis of the diagnostic profile of patients admitted to our center during 2018 and 2019.

Results: We found that, even without a focus on an specific disorder, $88 \%$ of our patients had a main diagnosis of personality disorder, with a big gap compared to other diagnoses (6,8\% psychotic disorders, $8,8 \%$ bipolar disorder, $9,8 \%$ OCD). In addition to this, the vast majority of patients undergoing treatment at our center have comorbidity, with $97 \%$ of patients with two or more psychiatric diagnoses.

Conclusions: Our findings could be explained by two main reasons. On the one hand, the difficult management of patients with these disorders on an outpatient setting and, on the other, the few specific resources that exist in our community for the treatment of patients with personality disorder.

Conflict of interest: No

Keywords: personality disorder; DAY HOSPITAL; comorbidity

\section{Psychoneuroimmunology}

\section{EPV1011}

\section{Pediatric acute-onset neuropyquiatric syndrome. An atypical case}

N. Serrano ${ }^{1 \star}$, M.D.C. Díaz Fernandez ${ }^{1}$, S. Guijarro ${ }^{1}$, M. Infante Sanchez De Lugarnuevo ${ }^{1}$, E. Landa ${ }^{1}$ and M.D.P. Alvites Ahumada ${ }^{2}$

${ }^{1}$ Hospital general de Tomelloso, Unidad De Salud Mental, Tomelloso, Spain and ${ }^{2}$ Complejo Hospitalario Mancha Centro, Unidad De Salud Mental Infanto Juvenil, Alcázar de San Juan (Ciudad Real), Spain ${ }^{*}$ Corresponding author.

Introduction: PANS is a clinical diagnosis given to children with dramatic neuropsychiatric symptoms, almost overnight, including obsessive-compulsive disorder(OCD), tics, and/or eating disorder. Children can get irritable and anxious, and struggle with school work. The cause of PANS is unknown in most cases, but is believed to be triggered by infections, metabolic disorders and other inflammatory reactions.

Objectives: Presentation a case with obsessive-compulsive disorder associated with recurrent urinary tract infections.

Methods: Case presentation The case concerns a woman 62 years old, without history of mental health, who presents autolysis ideation, with 5 attempts of autolysis, in last a year and a half. As a concomitant fact there were urinary tract infection of repetition, which was present in all episodes that the patient presented attempts of autolysis, with complete resolution of the picture after treatment of the infectious process. The patient was given a sublingual bacterian vaccine for urinary tract infections, and after a few months of administration, infections had disappeared, and episodes of autolysis hadn't occurred again. The patient now is in complete remission, although we maintenance treatment at low doses to avoid a relapse. Current treatment: Amisulpiride, mirtazapine, fluoxetine and lormetazepam.

Results: Patient who had recurrent attempts of autolysis, needed a lot of hospital admissions, recovered when a sublingual bacterial vaccine was administered for recurrent urinary infections.

Conclusions: -Diagnosis of OCD secondary to medical disease (recurrent urinary tract infections). -Always to rule out disease. -In this case, there may be recurrence of the clinic if the infection reappears. In this case we'll revaccination .

Conflict of interest: No

Keywords: PANS; PANDAS; OCD,recurrent urinary infection

\section{Psychopathology}

\section{EPV1017}

Association between the degree of irritability caused by trigger sounds and the presence of depression and anxiety in adults with different levels of misophonia

\footnotetext{
F.T. Antonia ${ }^{1 \star}$ and L. Giménez-Llort ${ }^{2}$

${ }^{1}$ Universidad de Barcelona, Departamento Psiquiatría, BARCELONA, Spain and ${ }^{2}$ Universidad de Barcelona, Departamento Psiquiatría, Barcelona, Spain

${ }^{\star}$ Corresponding author.
} 
Introduction: Misophonia can affect the individual's happiness, however, there is little evidence about its effects on the integral health. In addition, there is no consensus on diagnostic criteria, which hinders the correct identification of this neurobiological syndrome.

Objectives: This study aims to determine the association between misophonia, anxiety and depression in 88 adult misophonic patients (age of $44.83 \pm 14.05$ years, $65.5 \%$ were women).

Methods: The MAC2 Scale was used to measure misophonia and to establish four levels of severity: mild (scores of 11-22), moderate (23-34), severe (35-46) and extreme (47-54). Anxiety was measured with the Anxiety Questionnaire State-Risk (STAI) (Leibovich de Figueroa, 1991), while depression was assessed with the Tetradimensional Structural Questionnaire for Depression (Alonso-Fernández, 1986). The patients were exposed to 341 trigger stimulus (sounds and movements), and they rated the degree of irritability produced by each stimulus on a scale of 1 to 10 .

Results: The results showed a statistically significant correlation between the irritability produced by oral sounds and the patient's depression in all its dimensions, except for depressive mood. The irritability caused by kissing sounds and breathing sounds, especially from close friends, was statistically related to the patients' anxiety state $(\mathrm{R} 2=0.271)$ and anxiety trait $(\mathrm{R} 2=0.212)$. The telephone sound (as the vibration or tone) was significantly correlated with state-trait anxiety and with all dimensions of depression. Conclusions: These findings lead us to conclude that misophonia is related to depressive and anxious symptomatology, therefore it is important to take it into account during the patient's diagnosis and treatment.

\section{Conflict of interest: No}

Keywords: Misophonia; Anxiety; sounds; Dépression

\section{EPV1018}

Association between psychological well-being and the degree of irritability caused by trigger sounds in adults with different levels of misophonia

F.T. Antonia ${ }^{1 \star}$ and L. Giménez-Llort ${ }^{2}$

${ }^{1}$ Universidad de Barcelona, Departamento Psiquiatría, BARCELONA, Spain and ${ }^{2}$ Universidad de Barcelona, Departamento Psiquiatría,

Barcelona, Spain

${ }^{\star}$ Corresponding author.

Introduction: Misophonia is a neurobiological disorder that is not currently recognized in the DSM5, although our clinic experience of more than 30 years leads us to demonstrate that misophonia affects negatively the patients' quality of life. This study is innovative because nowadays there is little evidence on the understanding of this condition.

Objectives: Our aim is to identify the types of sounds that cause greater irritability in the patients, and its association with their psychological well-being (PWB)

Methods: A correlational design was carried out between 2015 and 2019, and 110 patients with four levels of misophonia were selected, measured with the MAC2 scale. The patients were exposed to 341 trigger stimulus (sounds and movements) grouped into 18 dimensions. The PWB was measured through the Psychological Well-being Scale of Sánchez-Cánovas (2013).

Results: The results showed that oral sounds were statistically significant and inversely associated to the patient's PWB $(\mathrm{R} 2=-0.238)$ and to the subjective PWB $(\mathrm{R} 2=-.0220)$. The body movements were correlated to the PWB related to the couple. The level of irritability produced by sounds and movements related to breathing and kissing was statistically correlated with occupational PWB $(\mathrm{R} 2=-0.194)$. Moreover, telephone sounds were significantly correlated with material PWB $(\mathrm{R} 2=-0.270)$. It was found that the level of misophonia was inversely associated to the patients' PWB.

Conclusions: Based on these findings, it is concluded that misophonia influences the PWB. Also, it is important to assess the irritability produced by the trigger stimulus, since it facilitates the patient diagnosis and treatment.

Conflict of interest: No

Keywords: Misophonia; trigger sounds; Adults; psychological well-being

\section{Psychopharmacology and pharmacoeconomics}

\section{EPV1067}

Efficacy of aripiprazole as a corrector of risperidoneinduced hyperprolactinemia.

\author{
C. Carrajo Garcia ${ }^{1 \star}, M$. Rodriguez De Lorenzo ${ }^{1}$, E. Alonso Sánchez ${ }^{2}$ \\ and C. Loeck De Lapuerta \\ ${ }^{1}$ HOSPITAL UNIVERSITARIO RAMON Y CAJAL, Psychiatry, \\ MADRID, Spain and ${ }^{2}$ HOSPITAL UNIVERSITARIO RAMON Y \\ CAJAL, Psychiatry, Madrid, Spain \\ ${ }^{\star}$ Corresponding author.
}

Introduction: A 33-year-old woman with a diagnosis of schizoaffective disorder, who goes to the mental health center of the day hospital to continuity of care. Background of two admissions (the first two years) in the Unit of Acute Psychiatry for psychotic episodes.

Objectives: Hyperprolactinemia (HPRL) has been considered until recently an inevitable side effect and inherent to antipsychotic treatment, and probably for this reason its clinical effects are poorly evaluated in the usual clinical practice. The purpose of this study is to refute this belief.

Methods: Upon arrival, the pharmacological treatment consists of risperidone $6 \mathrm{mg} /$ day. It has a good response to neuroleptic medication; However, given the appearance of undesirable side effects such as extrapyramidalism, weight gain $(10 \mathrm{~kg}$ in the last 6 months) and amenorrhea for 6 months (prolactin levels above the upper limit of normal: $40.2 \mathrm{ng} / \mathrm{dl}$ ) the change to oral aripiprazole and subsequently to its prolonged release form ( $400 \mathrm{mg} /$ day) with good tolerance is decided. The patient remained stable from the psychopathological point of view throughout the process.

Results: Virtually all antipsychotics drugs can produce HPRL, although to a different degree according to their profile of blocking and binding to D2 receptors, their relative limbic versus striatal selectivity, dose-response separation and interactions with other non-D2 receptors.

Conclusions: Aripiprazole is associated with a low prevalence of HPRL (3-5\%). Due to its partial agonism in D2 and because this union is prolonged, in monotherapy it has been seen that prolactinemia even decreases to values below normal.

\section{Conflict of interest: No}




\section{EPV1069}

Does long acting antipsychotic treatment reduce antipsychotic polypharmacy at discharge from hospitalization in psychiatric inpatient units?

C. Pérez Da Silva ${ }^{\star}$ E. Polonio Fuentes and D. Hernández Huerta University Hospital Ramon y Cajal, Psychiatry Department, Madrid, Spain

${ }^{\star}$ Corresponding author.

Introduction: Nowadays, second generation antipsychotic longacting injectable (SGA-LAI) use had widespread. Practical guidelines recommend then to ensure treatment compliance, and to avoid polypharmacy and high dose medication adverse effects. Yet there is little data about the real adherence to these recommendations.

Objectives: Prevalence description of polypharmacy with SGA-LAI and oral antipsychotics (OAPs) post-hospital discharge.

Methods: Retrospective study of hospital discharges on SGA-LAI treatment and frequency of concurrent OAPs treatment, between 01/01/2017-01/02/2018, in a tertiary hospital psychiatric inpatient unit (Spain). Loading oral dose in LAI-aripiprazole introduction is not included as polypharmacy. Descriptive analysis with the statistical program IBM SPSS v.22.

Results: 79 hospital discharges were included; 42 (53.2\%) were males, being the mean age 39.8 (SD: 10.238) years and average length of stay 15.23 (SD: 3.8 ) days. $44.3 \%$ were diagnosed of schizophrenia, $17.7 \%$ schizoaffective disorder, $10.1 \%$ bipolar disorder, $8.9 \%$ unspecified psychotic disorder, $7.6 \%$ delusive disorder, the rest $(11.4 \%)$ had brief psychotic disorder, substance-induced psychotic disorder, neurodevelopmental disorders, schizophreniform disorder and borderline personality disorder. 38(48.1\%) patients were treated with LAI-aripiprazole, 38 (48.1\%) had LAIpaliperidone and 3(3.8\%) LAI-risperidone. Regarding polypharmacy, 57 (72.2\%) had at least an OAPs, and 17 (21.5\%) had two different OAPs prescribed. $42 \%$ of discharges with OAPs had olanzapine prescribed, $21 \%$ quetiapine, $15.8 \%$ clotiapine, $14 \%$ risperidone, $12.3 \%$ haloperidol, $12.2 \%$ paliperidone, $5 \%$ aripiprazole, $3.5 \%$ asenapine, and 3.5\% levomepromazine.

Conclusions: Most of patients discharged with SGA-LAI had concurrent OAPs treatment. These data highlight the gap between research and prescribing practices in routine care and the need for further adaptation of clinical guidelines.

Conflict of interest: No

Keywords: Polypharmacy; hospitalization; long-acting injectable antipsychotics; oral antipsychotics

\section{EPV1071}

\section{Heart rate and QT interval in patients with clozapine vs controls}

P. Andres-Olivera ${ }^{*}$ E. Dominguez-Alvarez, R.S. GamonalLimcaoco, A. Bullón-Sáez and M.Á. Garzón-De Paz

CAUSA, Psychiatry, Salamanca, Spain

${ }^{\star}$ Corresponding author.

Introduction: Clozapine is the treatment of choice for patients with Refractory Schizophrenia. Sinus tachycardia is a side effect. There are different strategies for the control of the heart rate: decreasing the dosage, replacing for another antipsychotic or adding a negative chronotropic drug. In addition Clozapine is a drug that could prolong the QT.

Objectives: To study the length of the QT interval and the heart rate in beats per minute (bpm) in patients diagnosed with Refractory Schizophrenia treated with Clozapine. Comparing this group, to a control group of healthy people without any treatment. To determine changes in the heart rate or length of QT interval in both groups.

Methods: We review the electrocardiograms of 13 inpatients diagnosed with Refractory Schizophrenia admitted between June and July of 2019 in the Psychiatric Rehabilitation Unit undergoing monotherapy with Clozapine. Compared to13 healthy subjects. The registered heart rates and QT intervals were measured and corrected in both groups according to the Bazzet formula: QT ÖRR.

Results: 1 . In the patient group, the average measurement of QT obtained was more than $380.61 \mathrm{~mm}$; the corrected QT interval (QTc) was $419.76 \mathrm{~mm}$. In every case the QTc were within normal limits $(350-480 \mathrm{~mm})$. The average heart rate was $81.92 \mathrm{bpm}$. 2. In the control group the average QT length was $340,23 \mathrm{~mm}$; the QTc was $412.23 \mathrm{~mm}$. The average heart rate was $70.5 \mathrm{bpm}$

Conclusions: There are no significant differences in the QTc interval and heart rate observed in the sample of patients with Clozapine compared to the control group.

Conflict of interest: No

Keywords: clozapine; QT interval; Tachycardia; heart rate

Psychosurgery \& stimulation methods (ECT, TMS, VNS, DBS)

\section{EPV1103}

\section{Improvement strategies in the application of} electroconvulsive therapy

R. Martinez De Velasco ${ }^{1 \star}, \mathrm{P} . \mathrm{Nava}^{1}$, A. Rodríguez-Quiroga ${ }^{2}$, A. Matas ${ }^{1}$, C. Banzo ${ }^{1}$ and F. Mora ${ }^{1}$

${ }^{1}$ Hospital Infanta Leonor, Psychiatry, Madrid, Spain and ${ }^{2}$ Hospital Universitario Infanta Leonor, Psychiatry, Madrid, Spain ${ }^{\star}$ Corresponding author.

Introduction: Electroconvulsive therapy (ECT) is one of the main treatments available in psychiatry. Today the treatment is performed with devices that monitor the seizure induced by electrical stimulation by a short or ultra-short pulse wave. In this way, it has been possible to specify a minimum charge in electrical stimulation, with the consequent decrease in side effects. The main side effects are: Hemodynamic changes such as bradycardia, asystole and cardiac arrhythmias. There may also be episodes of desaturation, prolonged apnea, asthmatic exacerbation, airway obstruction in patients with apnea, bronchospasm pneumonia, bronchospasm and laryngospasm. Prolonged seizures, late seizures, nonconvulsive epileptic status) are rare and may go unnoticed if the EEG is not monitored, since they may not be accompanied by motor manifestations. The most frequent sequential effects are: headaches, nausea and fatigue. Cognitive effects are frequent during treatment: confusional syndrome, mania and amnesia.

Objectives: 1. Expose the modifications applied in our electroconvulsive therapy protocol. 2. Expose results obtained in our patients 
and the appearance of side effects. 3 To expose the reduction in the average stay of our patients.

Methods: Bibliographic review y statistical analysis of data collected from treatment by electroconvulsive therapy in patients admitted to the psychiatry unit of the Infanta Leonor University Hospital during the last two years.

Results: The results obtained show a decrease in secondary fects with special attention to respiratory, hemodynamic and cognitive complications.

Conclusions: The modifications made in our preparation protocol for electroconvulsive therapy reduce the side effects and reduce the average stay of patients.

\section{Conflict of interest: No}

Keywords: Electroconvulsive Therapy; mania; Dépression; schizophrenia

\section{EPV1107}

Electroconvulsive therapy in the treatment of comorbid obsessive-compulsive disorder and anorexia nervosa: a case report.

A. García Carpintero ${ }^{1 \star}$, B. Rodado León ${ }^{1}$, Í. Alberdi Páramo ${ }^{2}$ and A. García Recio ${ }^{1}$

${ }^{1}$ Hospital Universitario Clínico San Carlos, Instituto De Psiquiatría Y Salud Mental, Madrid, Spain and ${ }^{2}$ Hospital Clínico San Carlos., Institute of Psychiatry And Mental Health., Madrid, Spain

${ }^{*}$ Corresponding author.

Introduction: We present the case of a 19-year-old woman diagosed of restrictive anorexia nervosa since the age of 14 . She required eleven hospitalizations for nutrition in the last five years. She maintained a chronic BMI between 12 and 14 and suffers a chronic malnutrition state. In recent years, she developed mood worsening, suicidal thougths and obsessive-compulsive symptomatology with important fuctional impact. She was referred to our hospital in september 2019 due to a new weight loss episode. Given the lack of response of psicofarmacologic treatment we decided to iniciate electroconvulsive therapy (ECT).

Objectives: To study the efficacy of ECT in the treatment of obsessive-compulsive disorder (OCD) and anorexia nervosa.

Methods: We carried out a literature review of scientific papers in Medline data base. We used the followig terms: "obsessive compulsive disorder", "anorexia nervosa" and "electroconvulsive therapy". We considered english and spanish papers for the last 5 years.

Results: After twelve sessions of ECT our patient improved notably her mood state, eating habits and obsesions and compulsions were reduced. Literature research revealed that ETC could be efecctive in a significant percetage of patients diagnosed of OCD and also in some anorexia nervosa cases. One explanation of these findigs could be ECT affects the regulation of the cortico-limbic circuit, involved in both disorders.

Conclusions: ECT could be effective in mental disorders in which this tecnique is apparently not indicated for. More studies will be necessary to establish the mecanism of action and the profile of patients who coud benefit from this therapy.

Conflict of interest: No

Keywords: ECT; Electroconvulsive Therapy; obsessive-compulsive disorder; Anorexia nervosa

\section{Psychotherapy}

\section{EPV1124}

\section{A psychoanalytical way of re-constructing the speech in psychosis}

\author{
D. Gorgoli ${ }^{1 \star}$ and G. Mitropoulos ${ }^{2}$
}

${ }^{1}$ Constantopouleio General Hospital of Nea Ionia, Psychiatric

Department, Athens, Greece and ${ }^{2}$ Psychiatric Hospital of Attica, 5th

Department, Athens, Greece

${ }^{*}$ Corresponding author.

Introduction: It is very well known that psychoanalysis is not recommended for the psychotherapeutic treatment of psychotic patients. Indeed, the classical free association approach on the couch, is known to deconstruct the speech, whereas in psychosis we are struggling hard for the patient to re-organise his/her speech. Objectives: What we examine in this presentation, is the different approach of Lacanian psychoanalysis on psychotic patients. How is this approach different and why does there seem to be an advantage compared to the other classical analytical approaches? How does Lacan's work on the signifier play its part in the better understanding of psychosis?

Methods: We use here several examples to show that a patient with psychosis can get very well organised (and thus re-organised in psycotherapy) around a certain signifier and that this psychoanalytic approach can thus help the patient "anchor" his/her speech around this signifier and stop hallucinating. In order to do so, of course, we explain the basic lacanian terms of the signifier, the "other", how auditory hallucinations are regarded as speech hallucinations etc.

Results: We see here, how this different approach, based on the signifier can help the speech of the patient get re-organised, in addition to pharmacological treatment.

Conclusions: The way the brain works and how psychotherapy is working to create certain new paths in it, is a mesmerizing new world for psychiatry. This "speech alteration" that we present here, created by the "signifier" lacanian approach in psychosis, can therefore have significant both Clinical and scientific interest in the near future.

Conflict of interest: No

Keywords: psychosis; lacanian; Psychoanalysis; psychoanalytical

\section{Quality management}

\section{EPV1154}

Is there correlation between subjective patient satisfaction and psychiatric clinical evaluation in patients of day hospital?

P. García Vázquez ${ }^{\star}$, A. Serrano García and R. Gómez Martínez

Complejo Asistencial Universitario León, Psiquiatría, Leon, Spain ${ }^{\star}$ Corresponding author.

Introduction: The improvement perceived by the patients is a subjective measure of the psychic state, while clinical evaluation corresponds to an objective one performed by a psychiatrist. It is relevant to assess whether these parameters evolve in a common way after an intervention in Day Hospital. 
Objectives: Study the relationship between subjective improvement and clinical evaluation

Methods: This is a prospective study, which includes consecutive patients admitted to Day Hospital during 2018. Their objective clinical improvement was assessed by means of the PANSS and GAF scales at admission and discharge. Subjective clinical improvement was assessed using an anonymous Likert scale with a score between 1 and 7. Sociodemographic data and other satisfaction parameters were also collected. A statistical analysis was performed using Pearson's correlation.

Results: A total of 73 patients were included. The perception of improvement on the part of the patients is very high presenting average values close to the maximum. TCorrelation between subjective improvement and PANSS variation presented a Pearson value $0.008 ; \mathrm{p}=.957$ and with the GAF variation presented a Pearson correlation of $-0.066 ; \mathrm{p}=.578$ indicates that there is no significant correlation between the variables.

\begin{tabular}{llrr}
\hline \multicolumn{1}{l}{ CORRELATIONS } & & & \\
\hline \multirow{2}{*}{ STATE } & & STATE & VARIATION PANSS \\
\hline & PEARSON CORRELATION & 1 & 0,008 \\
\hline & Sig. (bilat) & & 0,957 \\
\hline VARIATION PANSS & PEARSON CORRELATION & 0,008 & 51 \\
\hline & Sig. (bilat) & 0,957 & 1 \\
\hline & N & 51 & \\
\hline
\end{tabular}

\begin{tabular}{llrr}
\hline CORRELATIONS & & & \\
\hline & & STATE & VARIATION GAF \\
\hline STATE & PEARSON CORRELATION & 1 & $-0,066$ \\
\hline & Sig. (bilat) & & 0,578 \\
\hline & N & 73 & 73 \\
\hline VARIATION GAF & PEARSON CORRELATION & $-0,066$ & 1 \\
\hline & Sig. (bilat) & 0,578 & \\
\hline & N & 73 & 73 \\
\hline
\end{tabular}

Conclusions: Clinical evaluation and subjective perception of improvement are completely independent parameters.

Conflict of interest: No

Keywords: PATIENT SATISFACTION; PSYCHIATRIC CLINICAL EVALUATION; DAY HOSPITAL

\section{Rehabilitation and psychoeducation}

\section{EPV1180}

\section{Patients perception of occupational therapy at} villaverde's day hospital.

\section{B. Márquez, T. Tomás and P. García-González}

Hospital de Día de Villaverde, Psychiatry, Madrid, Spain ${ }^{\star}$ Corresponding author.

Introduction: Villaverde's day hospital is an intensive psychotherapeutic treatment Center for adult patients (from 18 to 65 years old) with severe mental disorder. Our patients are included in different group therapies with a therapeutic community approach and a multidisciplinary view, with occupational therapy being an important part of their treatment. Working with patients through occupational therapy (OT) may change depending on the kind of patients treated and the type of units they are on.

Objectives: Our OT team has observed a change on the point of view of our patients regarding OT compared to previous experiences so we want to analyze this change in their perspective.

Methods: We performed individual interviews focusing on their experience with OT, enquiring about what motivated this change. Results: Through their testimonies, our patients describe a better experience with occupational therapy in our day hospital compared to other units they have been treated on.

Conclusions: We believe this change is due to different aspects. Usually our patients come into contact with occupational therapy in acute units, where the main focus is on occupational tasks. In our hospital, occupational therapy has its focus on the patients problems or biography, which they approach through a creative perspective.

Conflict of interest: No

Keywords: DAY HOSPITAL; Occupational therapy; rehabilitation

\section{Research methodology}

\section{EPV1188}

\section{Sensibility to change of mindfulness attention awareness scale (MAAS)}

A. Pinto García ${ }^{1 \star}$, A. Muñoz-Sanjose ${ }^{2}$, M.P. Vidal-Villegas ${ }^{3}$, R. Mediavilla ${ }^{4}$ A. García ${ }^{5}$, Á. Orosa-Duarte ${ }^{6}$ Á. Palao ${ }^{7}$, C. Bayon ${ }^{8}$, G. Lahera ${ }^{9}$ and B. Rodríguez-Vega ${ }^{4}$

${ }^{1}$ Fundación para la Investigación Biomédica del Hospital Universitario La Paz, Mental Health, Madrid, Spain; ${ }^{2} \mathrm{La}$ Paz University Hospital Institute for Health Research (IdiPAZ), Psychiatry, Clinical Psychology And Mental Health, Madrid, Spain; ${ }^{3}$ La Paz University Hospital Biomedical Research Foundation (F.I.B.H.U.L.P.), Psychiatry, Clinical Psychology And Mental Health, MADRID, Spain; ${ }^{4}$ La Paz University Hospital Research Health Institute (IdiPAZ), Psychiatry, Clinical Psychology And Mental Health, Madrid, Spain; ${ }^{5}$ Hospital Universitario La Paz, Psychiatry, Clinical Psychology And Mental Health, Madrid, Spain; ${ }^{6}$ La Paz University Hospital, Psychiatry, Madrid, Spain; ${ }^{7}$ HOSPITAL UNIVERSITARIO LA PAZ, Psiquiatria, MADRID, Spain; ${ }^{8}$ Autonomous University of Madrid (UAM), School of Medicine, Madrid, Spain and ${ }^{9}$ University of Alcala, School of Medicine, Alcalá de Henares, Spain

${ }^{\star}$ Corresponding author.

Introduction: The action mechanisms that explain how Mindfulness Based Interventions (MBIs) improve the well-being of people with mental disorders remain unclear (Alsubaie et al., 2017). This question leads us to ask how we measure mindfulness as a construct. Only two questionnaires are available nowadays in Spanish: the Five Facets Mindfulness Questionnaire (FFMQ) (Cebolla et al., 2012) and the Mindfulness Attention and Awareness Scale (MAAS) 
(Brown and Ryan, 2003). In the Spanish validation of the MAAS (Soler et al., 2012) the authors report a lack of sensibility to change that may render it inadequate to be used for the purpose of measuring the influence over mindfulness of a given intervention. Objectives: To test if a MBI (SocialMIND ${ }^{\circledR}$ ) increases mindfulness, as measured by MAAS.

Methods: A prospective single group clinical trial including participants with psychotic disorders was carried out. Mindfulness disposition was measured with the MAAS after 8 weeks of treatment.

Results: 33 participants were included. MAAS scores did not increase after SocialMIND ${ }^{\oplus}(\mathrm{t}=0.005, \mathrm{p}=1)$.

Conclusions: These results seem to support previous evidence which points towards the lack of sensibility to change of the MAAS (MacKillop et al., 2007; Soler et al., 2012). MAAS does not discriminate between non meditators and novice meditators. This is highly important in psychotic population because they have troubles acquiring habits, such as meditation. MAAS might be a valid instrument for studies where intense meditation practice is made, so a pre-post intervention change may be detected. More sensible instruments or new specific ones must be validated in Spanish.

Conflict of interest: No

Keywords: MAAS; Mindfulness; Schizophrenia Spectrum Disorder

\section{Schizophrenia and other psychotic disorders}

\section{EPV1232}

\section{Stressful life events and the onset of psychosis}

J. Gonçalves Cerejeira* ${ }^{\star}$ I.D.L.M. Santos Carrasco, M. Queipo De Llano, G. Guerra, A. Gonzaga, C. Capella, E. Rodríguez, M. De Lorenzo, M. Gómez, N. De Uribe and O. Santiago

Hospital Clinic of Valladolid, Psychiatry, Valladolid, Spain ${ }^{\star}$ Corresponding author.

Introduction: It is widely known that the exposure to stressful life events can trigger psychosis in vulnerable individuals. There are several models proposing the possible mechanisms underlying this relationship. Stress increases the cortisol level in the brain and therefore it could increase the level of dopamine receptors. On the other hand, it has been proposed that social disadvantage could increase the sensitivity to dopamine in the brain. Psychosis may also be explained as an exacerbated defense mechanism ("hyperconsciousness") in changing environments.

Objectives: To present a case of a first-psychotic episode during an Erasmus Program and to review the literature about stressful life events and psychosis.

Methods: We will present a case report and a literature review.

Results: We report a case of a 21-year-old woman, with no psychiatric nor substance abuse history. Erasmus student, living in a foreign European city for a month and a half. Her symptoms started after 3 weeks living abroad with confusion, thought blocking and persecutory and referential delusions. Physical exam, blood analysis and cerebral CT scan were normal. We started treatment with aripiprazole $20 \mathrm{mg}$ and lorazepam $3 \mathrm{mg}$ daily with clinical improvement in two weeks.

Conclusions: Changing environments can work as stressful life events that can trigger psychosis in vulnerable individuals. There is evidence in favor that stressful events in adult life may be involved in the onset of psychosis and our study supports these findings.
Conflict of interest: No

Keywords: stressful life envents; psychosis

\section{EPV1249}

Impact of a first psychosis episode program in clinical variables after two years of follow-up
M. Matilde ${ }^{1 *}$, E. García De Jalón ${ }^{2}$, M. Otero ${ }^{3}$, N. Pêreda ${ }^{4}$ and M.C. Ariz
${ }^{1}$ Servicio Navarro de Salud, Salud Mental Primeros Episodios, Pamplona, Spain; ${ }^{2}$ Complejo Hospitalario de Navarra, Servicio De Psiquiatría, Pamplona, Spain; ${ }^{3}$ OSASUMBIDEA, Salud Mental Primeros Episodios, Pamplona, Spain; ${ }^{4}$ osasunbidea, Salud Mental Primeros Episodios, Pamplona, Spain and ${ }^{5}$ Servicio Navarro de Salud, Servicio De Psiquiatría. Primeros Episodios., Pamplona, Spain ${ }^{*}$ Corresponding author.

Introduction: In Pamplona, Spain, there is an Early Intervention Program that has been providing assistance for First Psychotic Patients for the last two years.

Objectives: To analyze the longitudinal effects of the different interventions in clinical variables applied to 211 patients during two years of follow-up: CASH dimensions, substance abuse, antipsychotic type and dosage, remission rates, re-hospitalization rates and all-cause-of-treatment-dropout and DSM 5 diagnoses

Methods: We apply a standarized evaluation protocol to every patient at different times: premorbid, initial time, and every three months during follow up.

Results: The CASH positive dimension (delusions and hallucinations) had a mean score at baseline of 3.77 , showing an evident decline during the follow-up. The same happened with the disorganized dimension. We found no significant differences in the negative, motor and affective dimensions. Regarding remission rates from the 6 to the 24 months we found a decline in the rates, accordingly to the increase in re-hospitalization rates. Some of these cases were due to treatment dropout. The doses of antipsychotic treatment are lower at the end of follow-up Substance abuse rates are lower within the first six months, but after this time the percentage of non-consumers declines. The most frequent DSM 5 basal diagnosis is Brief Psychotic Episode, but during follow-up the diagnosis of Schizophrenia increase from $14.6 \%$ at baseline up to $46.2 \%$ at month 24 .

Conclusions: The Early Intervention Services improve, psychopathological dimensions and decrease re-hospitalization rates.

\section{Conflict of interest: No}

\section{EPV1258}

Symptomatology and addressability of patients with first episode psychosis at mental health services

V.I. Negrila ${ }^{1 *}$, A. Culici ${ }^{2}$, I. Negru ${ }^{3}$, R.T. Moldovan ${ }^{1}$, I.A. Rivis ${ }^{4}$ and C. Bredicean ${ }^{5}$

${ }^{1}$ Timișoara County Emergency Hospital, Clinic Of Psychiatry “eduard Pamfil”, Timisoara, Romania; ${ }^{2}$ Timișoara County Emergency Clinical Hospital, Clinic Of Psychiatry "eduard Pamfil”, Timișoara, Romania; 3"Victor Babeș" University of Medicine and Pharmacy, Faculty of Medicine, Timișoara, Romania; ${ }^{4}$ High-security Psychiatric Hospital Jebel, Psychiatry, Jebel, Romania and "Victor Babeș" University of Medicine and Pharmacy, Neuroscience, Timișoara, Romania ${ }^{\star}$ Corresponding author. 
Introduction: First episode psychosis is one of the most challenging subjects in Psychiatry because the symptoms can be complex, they are not specific, the prodrome varies and the consequences can be devastating if the patients don't receive the right treatment as fast as possible.

Objectives: The main goal was to evaluate the relationship between the symptomatology and the way of addressability at mental health services of a group of patients diagnosed with First Episode Psychosis. Methods: We observed 22 patients hospitalised in our Clinic of Psychiatry during September-December 2018 who were at the first contact with any Psychiatric Service and had the diagnosis of Acute and transient psychotic disorders( F23 on ICD 10). The following parameters were analyzed by us: gender, age, urban/rural, education, profession, marital status, way of addressability and clinic symptomatology. We divided the subjects in two groups: A patients brought by their families and $\mathrm{B}$ - patients brought by ambulance and police.

Results: The majority of subjects( $68,18 \%)$ were brought at the Hospital by ambulance and police but still at the initiative of their families. Subjects from group A showed: paranoid delusions(100\%), hallucinations $(71,43 \%)$, delusion of control $(71,43 \%)$ and expansive delusions $(85,81 \%)$. Subjects from group B showed: paranoid delusions(93.33\%), hallucinations(80\%), delusion of control(73,33\%) and expansive delusions(73,33\%). Statistically there are no major differences between the two groups regarding the symptomatology. Conclusions: The majority of subjects with First Episode Psychosis reach the Psychiatric Facilities brought by ambulance and police and less brought by their families. The dominant symptoms are paranoid delusions, hallucinations and expansive delusions.

Conflict of interest: No

Keywords: addressability; First Episode Psychosis; hallucinations; delusions

\section{EPV1263}

\section{Myxedema madness: psychosis may also happen. a review of symptoms through a case report.}

M. Pérez-Lombardo ${ }^{1 \star}$, A. González Castellary ${ }^{2}$, F.J. Gómez Beteta ${ }^{1}$, J. Pemán Rodríguez ${ }^{1}$, J.E. Ibáñez Vizoso ${ }^{1}$, M. Villanueva Gallego ${ }^{1}$, M. Jiménez Cabañas ${ }^{1}$, B. Rodado León ${ }^{1}$, G. Montero Hernández ${ }^{1}$ and G. Strada Herrera ${ }^{1}$

${ }^{1}$ Hospital Universitario Clínico San Carlos, Instituto De Psiquiatría Y Salud Mental, Madrid, Spain and ${ }^{2}$ Hospital Universitario La Princesa, Unidad Docente Centro, Madrid, Spain

${ }^{*}$ Corresponding author.

Introduction: Psychiatrists must always keep in mind the variety of organic mental disorders. Alterations in thyroid hormones are a common cause of psychiatric symptoms, most of them related to mood disorder. However, psychosis is not as well defined as a part of this disorders.

Objectives: The aim of this review is to bring closer to professionals this kind of manifestations, with a clinical example with a wide range of symptoms.

Methods: We conducted a review of the scientific literature on Pubmed, following a clinical case registered in our center. The search was focused on symptoms, its relation with blood levels of thyroid hormones and treatment.

Results: Thyroid disfunction are common and affect almost 5\% of the general population, with a female predominance. After Asher
(1949) created the concept of "myxedema madness", number of cases in literature has increased. Etiopathogenesis is still unknown, although several theories are defended. There is no correlation between the degree of thyroid dysfunction and the severity of the psychiatric symptoms manifested.

Conclusions: - Organic diseases can cause classic psychotic symptoms: hypothyroidism is one of them. - Etiopathogenesis of psychotic symptoms in hypothyroidism is still unknown. - Correlation was not found between the degree of thyroid dysfunction and the severity of the psychiatric symptoms. - Treatment consist on hormone replacement therapy, although some symptoms can remain in some cases. - Further investigations, as well as systematized reviews, are required to get a better knowledge of cases and get an earlier diagnosis.

Conflict of interest: No

Keywords: psychosis; myxedema; hypothyroidism; treatment

\section{EPV1353}

\section{What is behind the silence? a case report of mutism.}

B. Rodado-León ${ }^{1 \star}$, A. Bermejo Pastor ${ }^{1}$, A. García Carpintero ${ }^{2}$, M. Jiménez Cabañas ${ }^{2}$ and Í. Alberdi Páramo ${ }^{3}$

${ }^{1}$ Hospital Clínico San Carlos, Institute of Psychiatry And Mental Health, Madrid, Spain; ${ }^{2}$ Hospital Universitario Clínico San Carlos, Instituto De Psiquiatría Y Salud Mental, Madrid, Spain and ${ }^{3}$ Hospital Clínico San Carlos., Institute of Psychiatry And Mental Health., Madrid, Spain

${ }^{\star}$ Corresponding author.

Introduction: Mutism is a symptom which can be found as a part of different psychiatric disorders: selective mutism, schizophrenia and other psychotic disorders, hysteria, stupor, major depressive disorder, etc. In this work we present the case of a woman 50 years old who arrives to the hospital because see suffers from an atypical thoracic pain. Moreover, she is more focused on her religious devotion than usual, and verbalizes delusional ideas of prejudice. When the doctors suggest she should be evaluated by Psychiatry, she stops talking and doesn't respond to verbal or pain stimulation. Objectives: To describe a peculiar case of mutism, and to discuss the differential diagnosis which lead to an appropriate management of this case, with a rapid resolution.

Methods: The clinical history of the patient was collected guaranteeing her anonymity, and a comprehensive literature research was conducted via Pubmed, Web of Science, and Up to Date, using key words such as "mutism", "persistent delusional disorder", and "psychotic symptoms".

Results: Complementary exams were run to exclude organic pathology. The patient received treatment with two intramuscular injections of Zuclopenthixol acetate $50 \mathrm{mg} / \mathrm{ml}$ in a few days. Shortly after that, she started talking normally again, and the behaviour disorder associated to the delusional ideas improved.

Conclusions: According to the symptoms and vital history of the patient, the diagnosis was a persistent delusional disorder. However, we must not forget the importance of the organic evaluation, and the fact that a systematic anamnesis and differential diagnosis lead to a good outcome.

Conflict of interest: No

Keywords: mutism; persistent delusional disorder; Psychotic Symptoms 


\section{EPV1358}

Deterioration of oral and dental health in people with schizophrenia treated with antipsychotic medication

L. Urien $^{1 \star}$, N. Jauregizar ${ }^{1}$, B. Rodriguez ${ }^{2}$, A. Arnaiz ${ }^{2}$, L. Pérez ${ }^{2}$, R. Touzón ${ }^{2}$, J. López-Vicente ${ }^{3}$, A. Eguia Del Valle ${ }^{3}$, L. Pacheco ${ }^{2}$, J.J. Uriarte ${ }^{2}$, J.I. Landaluce ${ }^{2}$, M.C. Moreno ${ }^{2}$, M.A. Garay ${ }^{2}$ and T. Morera-Herreras ${ }^{1}$

${ }^{1}$ University of the Basque Country, Pharmacology, Leioa, Spain; ${ }^{2}$ Osakidetza, Basque Health Service, Biocruces Bizkaia Health Research Institute, Bizkaia Mental Health Network, Bilbao, Spain and

${ }^{3}$ University of the Basque Country, Stomatology Ii, Leioa, Spain

${ }^{\star}$ Corresponding author.

Introduction: Schizophrenia is probably the psychiatric disorder with the greatest impact on people's quality of life. Pharmacological treatment includes antipsychotics that are often effective for treating positive symptoms and have little impact on negative and cognitive ones. Oral side effects of antipsychotic medication, including alteration of the secretion of saliva, bruxism or oromandibular dyskinesia could compromise the oral health of these patients.

Objectives: To study the oral and dental health of people with schizophrenia attended in the Bizkaia Mental Health Network treated with antipsychotic medication.

Methods: A prospective multicentre descriptive study was carried out, including patients with a diagnosis of schizophrenia treated with antipsychotics $(n=69)$ and a control group $(n=40)$. An oral health status evaluation was performed, including DMF-T (decayed, missing, filled tooth) and CPITN (community periodontal index of treatment needs) indices, salivary flow measurement and yeast culture and identification. The diagnosis of the lesions detected in the oral mucosa was also established.

Results: Psychiatric patients had higher scores than control group in decayed and missing teeth (9.62 vs. 6.5), a higher need of periodontal treatment (CPITN index 2 vs. 1), and exhibited greater xerostomia scores (23 vs. 6 cases). Among the oral mucosa lesions, hyperkeratosis was described in $9 \%$ of psychiatric patients. No candidiasis was detected in any group.

Conclusions: The patients with schizophrenia in this study showed reduced oral health, which could be related to several factor such as limited access to dental care, higher use of alcohol, sugary drinks, tobacco but also to pharmacological treatment itself.

Conflict of interest: No

Keywords: schizophrenia; Dental; antipsychotic

\section{Sexual medicine and mental health}

\section{EPV1396}

\section{Am I hipersexual because I am bored?}

B. Martins Ramos*, D. Barbosa, A. Delgado and E. Osório

São João Hospital and University Center, Psychiatry And Mental Health Department, Porto, Portugal

${ }^{*}$ Corresponding author.

Introduction: Hipersexuality Disorder is being conceptualized in diverse ways, such as a sexual addiction, sexual compulsivity and sexual impulsivity. The proposed criteria for the DSM-5 characterize Hypersexual Disorder as a repetitive and intense preoccupation with sexual fantasies, urges, and behaviors, leading to adverse consequences and clinically significant distress or impairment in social, occupational, or other important areas of functioning. This approach follows a addiction model for the etiology of this disorder. Other authors have proposed an ABC model (Stein, 2008), which includes affective dysregulation, behavioural addiction and cognitive dyscontrol, creating a integrative theory explanatory for this disorder etiology. Boredom is considered a transitory affective/psychological state, to what some individuals may be more prone to, and can have a role in this model. Some authors' contruct proposal of hipersexuality posits boredom as an important dysphoric mood state triggering hypersexual behavior.

Objectives: This work aims to review the literature regarding the hypothesized correlation between boredom and hipersexuality.

Methods: Review of the literature and research studies published until 2019, using Pubmed and Google Scholar using key-words: hipersexuality, boredom, sexual addiction, sexual compulsivity, sexual impulsivity.

Results: Most studies showed a positive association between hipersexual behavior and boredom.

Conclusions: Hipersexuality Disorder can be explained through several factors and scientific community has not yet reached a conclusion. Regarding boredom, current literature identifies a link between it and hipersexuality. Although, further research is needed to better clarify and understand the relationship between the two concepts and other factors regarding hipersexuality etiology.

\section{Conflict of interest: No}

Keywords: sexology; hipersexuality; Addiction; boredom

\section{EPV1397}

\section{Ovotesticular disorder of sexual development and gender dysphoria - how far can we go?}

\author{
B. Martins Ramos ${ }^{1 \star}$, D. Barbosa ${ }^{2}$, A. Delgado ${ }^{1}$ and E. Osório ${ }^{1}$ \\ ${ }^{1}$ São João Hospital and University Center, Psychiatry And Mental \\ Health Department, Porto, Portugal and ${ }^{2}$ Sao Joao Hospital and \\ University Centre, Psychiatry, Porto, Portugal \\ ${ }^{*}$ Corresponding author.
}

Introduction: Ovotesticular Disorder of Sexual Development (DSD) refers to an extremely rare form of DSD, where adult gender identity is unpredictable and variable at birth and may evolve as the child grows.

Objectives: To review the literature regarding Ovotesticular DSD, gender atribution and gender dysphoria and reflect in how medical attitudes can impact patient's life's. To present a clinical case about an individual with $46 \mathrm{XX}$ karyotype and with Ovotesticular DSD, which was surgically interventioned twice, so her phenotype could match with her genotype. Afterwards presenting symptoms of gender dysphoria.

Methods: Review of the literature, using Google Scholar and Pubmed with the keywords: ovotesticular, DSD, gender dyshoria, medical intervention.

Results: Data from small case series suggests that patients reared in either sex can be satisfied with their sex assignment, but gender dysphoria also may occur. The clinical case is about a 16 years old adolescent with 46XX karyotype and born with bilateral female and male gonads. It was decided by the medical team and her parents for the removal of dysgenetic gonads in early chilhood and for a 
vaginoplasty when she was 14 years old. From two years now, she has described what seem to be a case of Gender Dysphoria.

Conclusions: Adult gender identity is difficult to predict in Ovotesticular DSD and therefore consensus and DSD advocacy groups have recommended that genital surgery be deferred until the child is old enough to confirm his or her gender identity. Medical intervention should be weighed very careful, unless it can be warmful.

Conflict of interest: No

Keywords: gender dysphoria; ovotestis; sexology; medical intervention

\section{Suicidology and suicide prevention}

\section{EPV1435}

\section{Passive and active suicidal ideation among psychiatry residents in spain: a cross-sectional study.}

D. Hernández-Calle ${ }^{1 \star}$, A. Cano Arenas ${ }^{1}$, D. Carracedo Sanchidrián ${ }^{1}$, R. Herranz Hernández ${ }^{2}$ and M.F. Bravo-Ortiz ${ }^{1}$

${ }^{1}$ Hospital Universitario La Paz, Psychiatry, Madrid, Spain and

${ }^{2}$ Hospital Clínico San Carlos, Preventive Medicine, Madrid, Spain

${ }^{*}$ Corresponding author.

Introduction: Psychiatry residents are constantly exposed to critical situations as without the experience to feel safe with their management, which it has been postulated that this differential characteristic compared could be associated with a high prevalence in suicidal ideation.

Objectives: To study the prevalence and characteristics of suicidal thoughts of Psychiatry residents in Spain.

Methods: We conducted a cross-sectional online survey directed to all medical residents in Spain. We analysed the proportion of passive and active suicidal ideation and its association with relevant Clinical and sociodemographic variables in the Psychiatry resident subpopulation.

Results: From a total of 94 Psychiatry residents, $13.83 \%$ (CI 95\% $6.85-20.81 \%)$ reported passive suicidal ideation $2.13 \%(0.00-7.48 \%)$ reported active suicidal ideation. These thoughts have the same prevalence among the whole residency program. The most influential association with passive suicidal thoughts were automedication (OR 6.81; 1.86-24.96) and attending to mental health consultation (OR 3.46; 0.95-12.58).

Conclusions: The prevalence of passive an active suicidal ideation among Psychiatry residents in Spain is 13.83\% (CI 95\% 6.85$20.81 \%$ ) and $2.13 \%$ (CI 95\% 0.00-7.48\%) respectively. These prevalences remained constant throughout the residency program. The most important risk factor founded were auto-medication and mental health consultation.

Conflict of interest: No

Keywords: Suicidal ideation; Medical Residents; Psychiatry residents

\section{EPV1439}

\section{Oddly happy after suicide attempt}

L. Santos Silva*, C. Almeida, J. Miranda, M. Barbosa, M. Duarte and J. Melim
Centro Hospitalar de Leiria, Serviço Psiquiatria E Saúde Mental, Leiria, Portugal

${ }^{\star}$ Corresponding author.

Introduction: Psychiatrists are used to dealing with patients who have attempted suicide in different contexts, with emergency services probably being the most common. Generally, these acts are usually performed by patients with depressive disorders, but may also be reactions to psychotic symptoms, or may arise after certain life events in people with maladaptive personality traits.

Objectives: The authors present the case of a 55-year-old man with previous psychiatric follow-up for depressive episodes, admitted to the emergency department after attempted suicide (AS) by hanging resulting in cardiorespiratory arrest. After stabilization, the patient was observed by psychiatrists presenting amnesia and symptomatology compatible with a first hypomanic episode, being hospitalized for stabilization and diagnostic clarification. The patient had had a psychiatric consultation with therapeutic adjustment of antidepressant medication the day before the AS, following the recent diagnosis of Brugada Syndrome. In personal history, there was no evidence of symptomatology compatible with manic or hypomanic episodes.

Methods: Medical interviews were carried out with the patient, as well as family interviews, essential in obtaining collateral information and previous personality assessment. Medical records by internal medicine colleagues were consulted, as well as the follow-up appointments after inward discharge.

Results: The patient was hospitalized and his symptoms improved following daily administration of sertraline, valproate and olanzapine. He was discharged after 10 days presenting adequate behavior, euthymic humor and no suicidal ideation.

Conclusions: Although cases of manic/hypomanic episodes secondary to cerebral anoxia described in the literature are rare, these evidences suggest that clinicians should be alert to all psychiatric manifestations that may arise after brain injury.

Conflict of interest: No

Keywords: Suicide; Dépression; brain injury; Hypomania

\section{EPV1441}

\section{Characterization of psychological pain in depressed patients}

A. Alacreu Crespo ${ }^{1,2 *}$, S. Guillaume ${ }^{1,2}$, P. Courtet $^{1,2}$ and E. Olie C $^{1,2}$ ${ }^{1}$ INSERM, U1061: Neuropsychiatry: Epidemiological And Clinical Research, Montpellier, France and ${ }^{2} \mathrm{CHU}$ Montpellier, Emergency Psychiatry And Acute Care, Montpellier, France ${ }^{\star}$ Corresponding author.

Introduction: Psychological pain is at core of suicidal behavior but its definition remains still debated.

Objectives: To investigate clinical characteristics of psychological pain associated with a recent suicide attempt $(<7$ days $)$ in depressed patients.

Methods: 371 depressed inpatients were divided into three groups: 178 recent suicide attempters, 101 past suicide attempters and 93 non attempters. Maximal psychological pain within the last 15 days was evaluated by a numerical scale. Psychopathology, level of depression, anxiety, affective lability and intensity, impulsivity and history of childhood trauma were evaluated.

Results: Recent suicide attempters were more prone to have high level of psychological pain than affective controls ( $\mathrm{OR}=1.33$ [1.17, $1.52])$ and past attempters $(\mathrm{OR}=1.19[1.04,1.36])$. Based on the 
median of psychological pain in recent suicide attempters, we considered high level of pain for a score $\geq 9$. In the whole sample, high psychological pain was positively associated with anxious and eating disorders, anxiolytics intake, physical and sexual abuses, as well as higher levels of depression, anxiety, affective intensity, impulsivity and physical pain. High psychological pain was negatively associated with lithium intake.

Conclusions: High psychological pain may help to improve detection of suicidal patients and provides therapeutic targets.

Conflict of interest: No

Keywords: Suicide; Psychological pain; Dépression

\section{EPV1464}

Suicide attempts and self-harm behavior in day hospital patients

\section{E. Rodríguez Muro and P. García-González ${ }^{*}$}

Hospital de Día de Villaverde, Psychiatry, Madrid, Spain ${ }^{\star}$ Corresponding author.

Introduction: Villaverde's day hospital for adults is an intensive psychotherapeutic treatment Center for adults with severe mental disorder. In this setting, self-harm and suicide attempts are behaviors that we have to address regularly with our patients. In addition, these behaviors are becoming more and more frequent in the general population, adding an important amount of visits to emergency units and generating suffering for the people who present them and for their loved ones.

Objectives: We want to study the presence of self-harm behavior and suicide attempts among our patients. In order to understand its individual differences to address them properly with our patients, we want to know its proportion and try to analyze the different meanings and causes of these conducts individually.

Methods: We made a qualitative analysis of self-harm behavior and suicide attempts in patients admitted to our center in the past two years and analyzed individually cases that presented them.

Results: $56.8 \%$ of patients had one or more suicide attempts and $36.3 \%$ had self-harm behavior of some kind. We also observed that there are different meanings, functions and causes of these behaviors in different patients. It is important to point out that sometimes it is difficult to establish the difference between self-harm behavior and suicide attempt.

Conclusions: An individualized approach to self-harm behavior and suicide attempts is necessary, giving patients the time and space necessary to understand and translate these behaviors in order to reduce or eliminate them.

Conflict of interest: No

Keywords: Suicide; Self-harm; DAY HOSPITAL

\section{Training in psychiatry}

\section{EPV1476}

Antipsychotics complications monitoring: evaluation of practice among young psychiatrists

S. Ennazk ${ }^{\star}$, M. Abrache, K. Akebour, I. Adali, F. Manoudi and F. Asri
Equipe de recherche pour la santé mentale, service de psychiatrie, Hôpital Ibn Nafis, CHU MARRAKECH, Psychiatry, Marrakech, Morocco

${ }^{*}$ Corresponding author.

Introduction: Antipsychotics are widely used in psychiatry for different pathologies. They remain a highly potentially dangerous family of medications. The NICE has made recommendations regarding the monitoring of complications of antipsychotics.

Objectives: To evaluate adherence of young psychiatrists to NICE recommendations for evaluating complications of antipsychotics. To improve awareness of young psychiatrists and their practice concerning this issue. To establish a system of prevention of complications of antipsychotics in Ibn Nafis hospital.

Methods: A transversal study was conducted. À questionnaire was given to 40 young psychiatrists (interns and residents in psychiatry). The questionnaire included questions about four categories : lifestyle physical activity Weaning tobacco precautions while prescribing antipsychotics In this last category, the guidelines of NICE were used as a reference.

Results: $50 \%$ of young practitioners give their patients advice concerning lifestyle, but only $10 \%$ refer them to an actual program of dietetic education or physical activity. $55 \%$ of patients talk about weaning tobacco to their patients. $25 \%$ of them refer to a specialist in addictology. $60 \%$ of young psychiatrists never measure their patients weight prior to introducing antipsychotics. $10 \%$ ask for a lipidic screening in all patients, while $70 \%$ ask for a glycemic screening before introducing antipsychotics. $80 \%$ maintain an annual screening of glucose. $60 \%$ maintain a screening for abnormal movements during follow-up.

Conclusions: There is a wide disparity between practitioners. Older ones are more precautious than younger ones. A unified practice should be encouraged through pre-established screening vouchers.

Conflict of interest: No

Keywords: complications; Young psychiatrists; antipsychotics; monitoring

Women, gender and mental health

\section{EPV1490}

Be born woman, be trans, have a mental health diagnosis: when stigma puts your life at risk.

\author{
L. Fontecha Banegas ${ }^{1 \star}$, I. Cuevas ${ }^{2}$ and I. Moreno Alonso ${ }^{1}$ \\ ${ }^{1}$ Hospital Universitario Principe de Asturias, Psiquiatria, Alcala de \\ Henares, Spain and ${ }^{2}$ Hospital Universitario Principe de Asturias, \\ Psiquiatria, Alcalá de Henares, Spain \\ ${ }^{*}$ Corresponding author.
}

Introduction: The case of a 22-year-old patient who comes to the Emergency Department due to general malaise, apathy and generalized psychomotor inhibition is presented. Initially, the patient was not medically assessed, since the medical team assumed that the presented clinic was compatible with symptoms of the dissociative sphere, so that they contacted to psychiatry team. Objectives: To think on Mental Health's stigma at the hospital setting and its possible (and sometimes potencially harmful) consequences. 
Methods: During his stay in the Emergency Department, blood test, cranial CT, abdominal X-ray, urine toxicity test, pregnancy test, neurological and psychopathologic examination, and lumbar pucture were performed.

Results: Neutrophilia (increasing in successive measurements). Hypodense image on the left frontal hemisphere; colic frame full of feces. Generalized hypotonia and frontal release reflexes. Very frugal speech with long response latency. Hyperproteinorraquia.

Conclusions: Any clinical condition suspected of having an organic origin (regardless of whether or not the patient has a diagnosis of Mental Health), requires adequate and complete organic screening. Failure to do so can have serious consequences.

Conflict of interest: No

Keywords: Stigma; mental health; organic screening; consequences

\section{EPV1504}

\section{Use of oral aripiprazole in a pregnancy woman with} schizophrenia. Case report.

M. Rodriguez De Lorenzo ${ }^{1 \star}$, C. Carrajo Garcia ${ }^{2}$, C. Loeck De Lapuerta $^{3}$, E. Alonso Sánchez ${ }^{2}$ and P. Del Sol Calderón ${ }^{4}$

${ }^{1}$ Hospital Universitario Ramon y Cajal, Psychiatry, Madrid, Spain; ${ }^{2}$ HOSPITAL UNIVERSITARIO RAMON Y CAJAL, Psychiatry, MADRID, Spain; ${ }^{3}$ RAMON Y CAJAL UNIVERSITY HOSPITAL, Psychiatry, MADRID, Spain and ${ }^{4}$ HOSPITAL UNIVERSITARIO PUERTA DE HIERRO MAJADAHONDA, Psychiatry, MADRID, Spain

${ }^{*}$ Corresponding author.

Introduction: A 38-year-old woman with a diagnosis of Paranoid Schizophrenia who, while being treated with oral Aripiprazole, becomes pregnant. No medical history of interest. Psychiatric history: one hospital admission in 2009, subsequent follow-up in day hospital for 3 months, and outpatient follow-up without relapses until today. Current treatment: Aripiprazole $20 \mathrm{mg}$ (half a tablet for breakfast and half a meal).

Objectives: The aim of this case report is to show that Aripiprazole at low doses could be use in pregnancy, always under medical supervision and with informed consent, in psychotic disorders.

Methods: Case report and literature review

Results: Being under regular outpatient follow-up and under treatment with $20 \mathrm{mg}$ of Aripiprazole orally, the patient becomes pregnant. Risks and benefits of continuing or discontinuing the treatment were explained, and she finally accepts, by informed consent, to continue with the treatment. The delivery was completed and without complications. She has decided to start artificial lactation because she is continuing the prescribed treatment.

Conclusions: There are no trials in well-controlled and adequate pregnant women with Aripiprazole. In this case, we contacted SITTE (Service of telephone information of teratogens in Spain) who informed both, patient and therapist, of the possible risks and benefits, and together decided their continuation. Artificial lactation is recommended in this cases.

Conflict of interest: No

Keywords: aripiprazole; schizophrenia; lactation; Pregnancy

\section{EPV1505}

Use of oral olanzapine in a pregnancy woman with bipolar disorder. Case report.

M. Rodriguez De Lorenzo ${ }^{1 \star}$, C. Loeck De Lapuerta ${ }^{2}$, E. Alonso Sánchez ${ }^{3}$, C. Carrajo Garcia ${ }^{3}$ and P. Del Sol Calderón ${ }^{4}$

${ }^{1}$ Hospital Universitario Ramon y Cajal, Psychiatry, Madrid, Spain; ${ }^{2}$ RAMON Y CAJAL UNIVERSITY HOSPITAL, Psychiatry, MADRID, Spain; ${ }^{3}$ HOSPITAL UNIVERSITARIO RAMON Y CAJAL, Psychiatry, MADRID, Spain and ${ }^{4}$ HOSPITAL UNIVERSITARIO PUERTA DE HIERRO MAJADAHONDA, Psychiatry, MADRID, Spain ${ }^{*}$ Corresponding author.

Introduction: 38-year-old woman diagnosed with Bipolar disorder who becomes pregnant on treatment with Levomepromazine and at week 23 it is changed to Olanzapine. No medical history of interest. Psychiatric history: 3 hospital admissions, the last one was last year (serious episode with psychotic symptoms), since then, she takes Levomepromazine $75 \mathrm{mg}$ a day and now she receives an outpatient follow-up.

Objectives: The aim of this case report is to show that Olanzapine at low doses could be use in pregnancy, always under medical supervision and with informed consent, in psychotic disorders.

Methods: Case report and literature review

Results: Patient and psychiatrist contact SITTE (Telephone information service of teratogens in Spain) who suggests a change to another antipsychotic. Risks and benefits are evaluated, and it is decided to switch to Olanzapine in the 23 rd week of gestation. Since then, she has been taking half a tablet of Olanzapine $25 \mathrm{mg}$ orally. The birth has not had complications. The patient has decided to breastfeed her baby.

Conclusions: Currently, Olanzapine is classified as FDA category C, despite not having adequate and controlled studies in humans. Nevertheless, it is accepted to keep the treatment during pregnancy in cases of severe Bipolar disorder such as this, since the benefits, both for the mother and the fetus, are greater than the possible risks. Artificial breastfeeding is recommended in these cases.

Conflict of interest: No

Keywords: breastfeeding; Bipolar disorder; olanzapine; Pregnancy

\section{Schizophrenia and other psychotic disorders}

\section{EPV1570}

Hospitalizations of patients with severe schizophrenia treated in a community based, case managed program vs. standard care. A ten-year follow-up

\section{S. Diaz-Fernandez ${ }^{*}$ and J.J. Fernandez-Miranda}

Spain

${ }^{*}$ Corresponding author.

Introduction: Case management has been shown to be successful in reduction of hospitalizations and people leaving treatment.

Objectives: To know the treatment adherence and the psychiatric hospitalizations of patients with severe schizophrenia (GCI-S $\geq 5$ ) 
before (standard treatment) and during treatment in a community based, case managed program. And also the role of oral or longacting injectable antipsychotic medication.

Methods: Observational study, mirror image, of ten years of followup and ten retrospectives, of patients with severe schizophrenia in a community based program, with integrated pharmacological and psychosocial treatment and intensive case management $(\mathrm{N}=344)$. Reasons for the Program discharge and psychiatric hospital admissions were recorded ten years before and during treatment. And also the antipsychotic medication prescribed.

Results: After 10 years $12.2 \%$ of the patients were voluntary discharges (In previous standard treatment: $84.3 \%$ ). CGI-S at baseline was $5.9(0.7)$. After ten years $51.7 \%$ of patients continued under treatment $(\mathrm{CGI}-\mathrm{S}=3.9(0.9) ; \mathrm{p}<0.01) ; 19.3 \%$ were medical discharged (CGI-S=3.4(1.5); $\mathrm{p}<0.001$ ); and 12.2 were voluntary discharges. Forty patients died during follow-up, five of them by suicide.The percentage of patients with hospital admissions and the number of admissions due to relapses decreased after beggining in the Program $(\mathrm{p}<0.0001)$, and as well the involuntary ones ( $\mathrm{p}$ $<0.001)$. To be on long-acting injectable antipsychotic treatment was related to these results $(\mathrm{p}<0.0001)$.

Conclusions: Treatment of patients with severe schizophrenia in a integrated, case-managed community-based program achieved higher retention, and was effective in reducing psychiatric hospitalizations, compared to the previous standard treatment. To be treated with long-acting injectable antipsychotics was clearly linked to these outcomes.

Disclosure: No significant relationships.

Keywords schizophrenia; case management; treatment adherence; hospitalization

\section{Symposium}

Immune alterations in early psychosis: new goals for diagnosis and treatment

\section{S046}

Common genes in neurodevelopment and immuneinflammatory pathways impacted in schizophrenia?

N. Ramoz ${ }^{\star}$ and P. Gorwood ${ }^{*}$

INSSERM, Institute of Psychiatry and Neuroscience Of Paris, Paris, France

${ }^{\star}$ Corresponding author.

Introduction: There are evidence suggesting involvements of genetics factor in the predisposition of schizophrenia. Furthermore, recent researches, including epidemiological studiesn also indicate that immune-inflammatory mechanisms could be risk factors in the development of psychosis to schizophrenia.

Objectives: Taking into advantage of the recent schizophrenia consortium studies using omic tools, including genetics, transcriptomics and epigenomics, we want to characterize genetic profiles of genes involved in neurodevelopment and immune-inflammatory pathways that should play a role in the pathophysiology of schizophrenia. Methods: Using the pan-genomics studies of genetics, the characterization of copy number variants, genes involved in the neurodevelopment, as well as, genes of the immune system, could be associated to psychosis and schizophrenia. The functional analysis of gene expression by the transcriptomics from blood to brain of the patients, as well as, epigenetic studies reinforce these observations. Finally, the pathway and network analyses should help us to develop models of integration of biological system altered in schizophrenia. Results: The characterization of copy number variants associated to schizophrenia or psychosis allowed to identify common genes involved in neurodevelopment and immune-inflammatory pathways. Furthermore, genome-wide association studies and networkbased analysis confirm an enrichment of variants associated with schizophrenia of genes involved in brain or immunity pathways. Genome-wide expression and Cross-disorder analysis also conclude to these observations.

Conclusions: These convergent observations and with tools of omics techniques, we could now propose a help for the diagnostic for a precision medicine in schizophrenia and also to adapt specifically the treatments from one patients to another.

Conflict of interest: No

Keywords: neurodevelopment; genetics; schizophrenia; psychosis

\section{Mental health and the transition from child to adult mental health services in europe}

\section{S098}

Improving transitions in europe: lessons from the milestone project

H. Tuomainen ${ }^{1 \star}$, J. Warwick ${ }^{2}$ and S. Singh ${ }^{1}$

${ }^{1}$ University of Warwick, Warwick Medical School, Mental Health and Wellbeing, Coventry, United Kingdom and ${ }^{2}$ University of Warwick, Warwick Medical School, Clinical Trials Unit, Coventry, United Kingdom

${ }^{*}$ Corresponding author.

Introduction: Young people reaching the upper age boundary of their Child and adolescent mental health service (CAMHS) face transition to adult services, if they need continued treatment. In many European countries, transition is not well managed and a large proportion of young people experience discontinuity of care after the transition boundary (TB). In the MILESTONE project, we surveyed CAMHS provision at the TB and professional training linked to transition; examined biopsychosocial outcomes of young people who have reached the TB of their CAMHS in eight European countries; and implemented "Managed Transition" to improve their outcomes.

Objectives: We share key findings from the various MILESTONE studies to illustrate the complexity of problems and solutions at the mental health service boundary in Europe.

Methods: Data was collected via systematic literature reviews; bespoke surveys to CAMHS professionals, experts and other stakeholders; a longitudinal cohort study with a nested clinical trial in eight EU countries involving over $1000 \mathrm{CAMH}$ service users, their parents/carers, and clinicians; focus groups and interviews. The project had active and intensive participation of young people from two countries.

Results: CAMHS provision and delivery of specific services vary significantly between the 28 EU countries. Commonly, 25 to $49 \%$ of service users need transitioning to AMHS. Training of CAMHS professionals in transition has only recently been considered. Key trial findings on Managed Transition will be presented. 
Conclusions: Findings from MILESTONE are complex and already being used to inform policy and mental health service development in Europe.

Conflict of interest: No

Keywords: mental health services; young people; Transition; Europe

\section{Can innovative technologies optimise mental health care? Some shining examples}

\section{S168}

The smart-crisis project: ecological risk assessment in suicidal patients

\author{
J. Lopez-Castroman ${ }^{1,2,3}$ \\ ${ }^{1}$ Inserm, Unit 1061, Montpellier, France; ${ }^{2}$ University of Montpellier, \\ Psychiatry, Montpellier, France and ${ }^{3}$ Nimes University Hospital, \\ Psychiatry, Nimes, France
}

The Prevention of suicidal behavior is a public health priority, but new tools are needed to intervene with those who need it most. These tools are currently available thanks to new technologies. Suicide attempters, or patients with mental disorders that are disproportionately at risk of attempting suicide, are usually assessed through clinical interviews, sometimes using self-rated or clinicianrated questionnaires. These methods have proven a limited capacity to predict the risk of future attempts. Situational biases, such as the clinical setting, probably influence this lack of accuracy since the assessment takes place in a very different context compared to everyday life for the patients. To avoid such problems, momentary ecological assessment provides a way to assess patients longitudinally, in real-time and in their natural environment. We will present the first results of a research project that assesses suicidal patients using two mobile applications. The first of them allows patients to send daily reports on their condition and the second obtains passive data about the activity of the smartphone. The particularity of this project is the evaluation of basic physiological functions such as sleep or appetite, which are easily identifiable without causing stigma. These markers could predict in real time an increased risk of suicide in a vulnerable population and allow rapid intervention.

Conflict of interest: No

Keywords: digital phenotype; ecological momentary assessment; ehealth; suicidal behavior

\section{Workshop}

Secondary prevention of suicide

\section{W006}

\section{The role of antidepressants in the secondary prevention} of suicide

\footnotetext{
J. Lopez-Castroman ${ }^{1,2,3}$

${ }^{1}$ Nimes University Hospital, Psychiatry, Nimes, France; ${ }^{2}$ Inserm, Unit 1061, Montpellier, France and ${ }^{3}$ University of Montpellier, Psychiatry, Montpellier, France
}

Several trials have recently shown that a poor response to antidepressant treatment predicts the emergence of new suicidal ideas and attempts. This effect seems to be associated to an excess in the antidepressant dosage and to a young age of the patients. Inversely, new data also suggests that suicidal depressed patients (independently of comorbidity and type of treatment) respond less well when an antidepressant treatment is prescribed. The role of activation syndrome, or antidepressant-induced jitteriness/anxiety syndrome, needs also to be discussed since specific symptoms seem to be particularly associated with the risk of emergent suicidal ideas or behavior. Overall, this presentation will review the evidence in clinical samples of suicidal patients about the preventive effects of antidepressant treatment.

Conflicts of Interest: No

Keywords: Antidepressant response; activation syndrome; adverse effects

\section{An exciting time for research for early career psychiatrists: methods and perspectives}

\section{W059}

Social media analysis for psychiatric research: mental health infodemiology in twitter

\section{Alvarez-Mon}

University Hospital Infanta Leonor, Department of Psychiatry, Madrid, Spain

In recent years, the internet has radically changed how most people communicate, share and seek out information regarding health and medical conditions. The utilization of online data for health care purposes has led to the development of an emerging field defined as Infodemiology. Twitter is one of the most popular and widely used platforms of social media. Different players in medicine have realized Twitter's potential for acquiring and distributing medical information. The utilization of Twitter data has enabled researchers to study health related attitudes towards behaviors and diseases, predict the incidence of communicable and no communicable diseases or have an insight of patient's medical experience. Moreover, the analysis of tweets about psychiatric disorders is a recent area of study for understanding the sentiments of society, patients and health players. This talk will discuss Twitter's potential as a tool for developing mental health interventions with a clinical point of view. The speaker has participated in several studies in this field.

Conflicts of Interest: No

Keywords: Infodemiology; Twitter; mental health

\section{The complexity of bipolar disorders}

\section{W074}

Neuropsychological impairment in patients with bipolar disorder

A. Martinez-Aran 
Clinical Institute of Neuroscience, Hospital Clinic, University of Barcelona, IDIBAPS, CIBERSAM, Bipolar and Depressive Unit, Barcelona, Spain

Patients with bipolar disorders (BD) suffer from cognitive deficits across memory, attention, processing speed and executive function during periods of remission with a huge impact on functional recovery. The association between cognitive performance and psychosocial functioning has led to emergence of neurocognition as a new treatment target. Moreover, cognitive impairment in the remitted phase of $\mathrm{BD}$ is on average of a moderate effect size. However, recent studies indicate substantial cognitive heterogeneity in remitted $\mathrm{BD}$ patients, and most importantly, patients with either global or selective cognitive impairments showed lower levels of functioning and reported poorer quality of life. Cognitive impairments may reflect neurodevelopmental factors, such as genetic susceptibility and illness progression, including potential neurotoxic effects of recurrent episodes as well as chronic medication use. It is important to identify patients with persistent cognitive deficits, to characterize the pattern of their impairments, and to implement strategies for remediating these deficits to improve not only the clinical management and neurocognitive impairment but also functional outcome. Therefore, neurocognitive impairment needs to be considered a therapeutic clinical target, from early stages, in order to improve both psychosocial functioning and quality of life of patients with $\mathrm{BD}$. Despite the increase of research investigating new pharmacological and non-pharmacological treatments over the last decade, no robust evidence of therapeutic interventions targeting cognitive deficits is currently available, due to insufficient data, and further research is needed to be largely explored and draw firm conclusions.

Disclosure: Grants: CIBERSAM, the Spanish Ministry of Science, Innovation and Universities, and the Instituto de Salud Carlos III (FIS PI15/00330; PI18/00789), and PERIS (SLT006/17/00177).

Keywords: Bipolar disorder; neurocognition; functioning 